\title{
DO FOLIAR ANTHOCYANIN PIGMENTS IN HOROPITO (PSEUDOWINTERA COLORATA) FUNCTION AS VISUAL SIGNALS TO DETER INSECT HERBIVORES?
}

BY

IGNATIUS JEPPE MENZIES

\begin{abstract}
A thesis
submitted to the Victoria University of Wellington in fulfilment of the requirements for the degree of Doctor of Philosophy
\end{abstract}

Victoria University of Wellington (2013) 


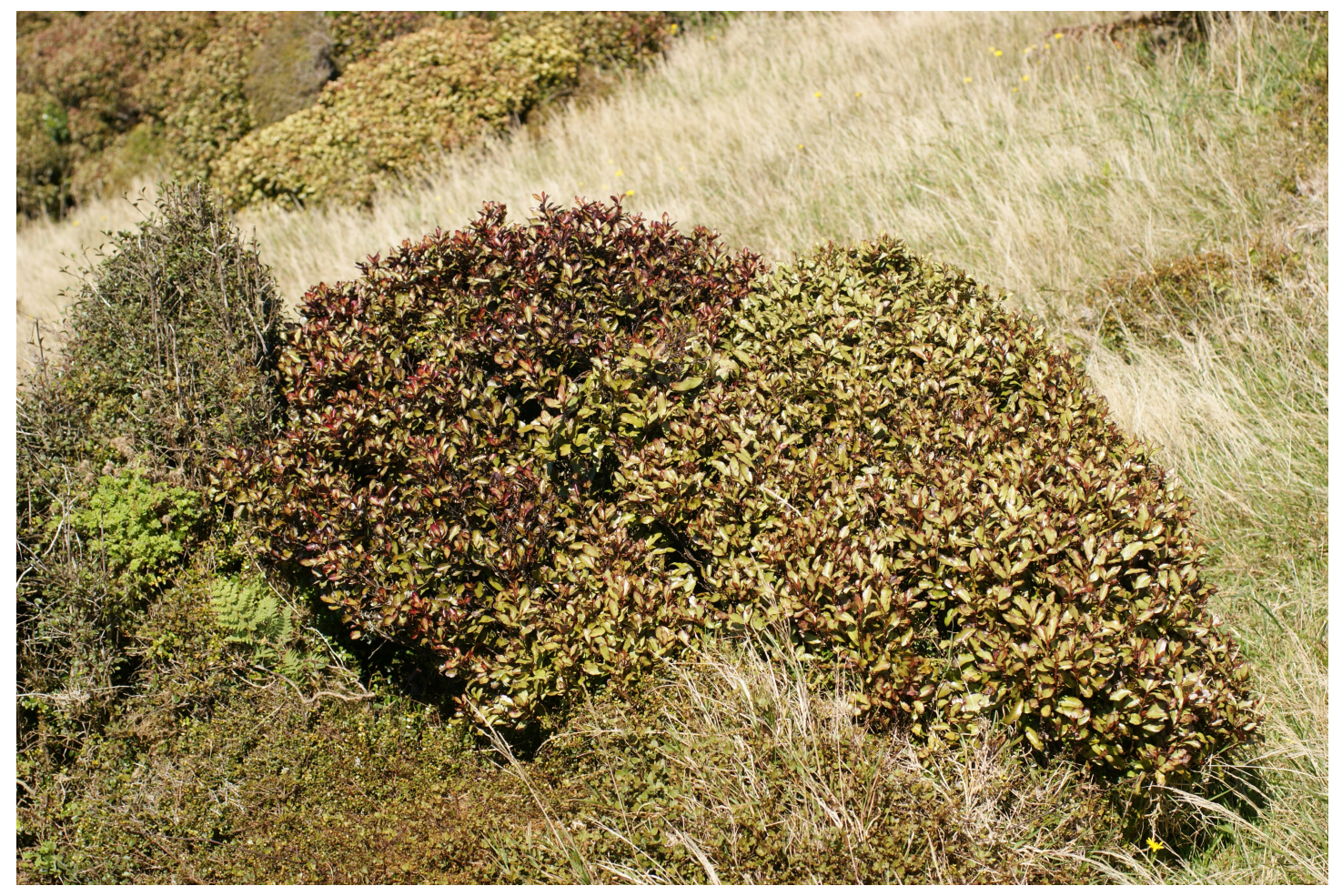

Matched pair of red and green P. colorata at Belmont Trig, Wellington, New Zealand 


\section{ABSTRACT}

Anthocyanin pigments are synthesised in the leaves of many plants, however the adaptive significance of these pigments is not entirely understood. It has been postulated that their red colours may function as visual signals through coevolution between herbivorous insects and their host tree species, though the hypothesis lacks solid empirical evidence. I investigated the leaf signalling hypothesis using Pseudowintera colorata, focusing on five areas:

1) I exploited the natural polymorphism in leaf colour of $P$. colorata to test the predictions that (i) bright leaf colour is a reliable signal of a plant's defensive commitment; (ii) insects in the field avoid trees that are brightly coloured; and (iii) the trees with the brightest leaves will have higher fitness. Relative to green leaves, redder foliage contained higher concentrations of polygodial, a sesquiterpene dialdehyde known to have strong antifeedant properties, and incurred less insect feeding damage. Redder trees hosted fewer Ctenopseustis spp. leafroller larvae than neighbouring matched green trees. Contrary to the predictions of the leaf signalling hypothesis, there was no difference in any of the measured fitness parameters between red and green trees, indicating that the leaf colour polymorphism in P. colorata is stable.

2) Many insects are sensitive to volatile organic compounds (VOCs), however the role of VOCs in plant-herbivore signalling has not been investigated. I analysed VOCs released from undamaged, herbivore- and mechanically-damaged red and green leaves of $P$. colorata, and the olfactory preferences of brownheaded leafroller (C. obliquana) larvae. While the VOC profiles of browsed and unbrowsed leaves were statistically distinguishable, the VOC profiles released from intact, herbivore-, and mechanically-damaged $P$. colorata leaves did not reliably identify leaf colour Moreover, naïve and experienced C. obliquana larvae displayed no preference for the volatiles from mechanically damaged red or green leaves. Therefore, I concluded that VOC compounds are not likely to play a large role in mediating insect herbivore-plant interactions in P. colorata. 
3) Studies of leaf signalling rarely consider the influence of the light-absorbing properties of non-green pigments upon photosynthesis. I compared the photosynthetic and photoinhibitory responses of red and green leaves from matched, neighbouring pairs of $P$. colorata of contrasting colour. Redder $P$. colorata leaves in the field had a lower maximum photosynthetic assimilation rate than matched green leaves from neighbouring trees. However, I was unable to detect any measurable advantage in terms of photoprotection in the red $P$. colorata leaves as indicated by chlorophyll fluorescence profiles. My results indicate that the presence of anthocyanin pigments within non-senescing leaves may impose a slight photosynthetic cost to the plant.

4) I used literature searches, field surveys and laboratory bioassays to identify which invertebrate herbivores are most likely to participate in leaf-signalling interactions with $P$. colorata. Feeding preference bioassays showed that brownheaded leafrollers (C. obliquana and C. herana) and Auckland tree weta (Hemideina thoracica) preferentially consumed leaf material from green than red $P$. colorata leaves. Results from these bioassays, combined with my field surveys suggest that Ctenopseustis spp. leafroller larvae are the most likely coevolution partners for $P$. colorata.

5) There is a well-established link between nitrogen deficiency and leaf reddening. Additionally, leaf nutrients can influence foraging behaviour and performance of insect herbivores. I measured $\mathrm{N}$ and $\mathrm{C}$ contents of leaves from neighbouring matched pairs of red and green $P$. colorata. There were no significant differences in the amounts of, or ratio between, $\mathrm{N}$ and $\mathrm{C}$ between matched red and green leaves. This result indicates that differences in colour and herbivory among $P$. colorata leaves are not attributable to differences in leaf nutrients.

Taken together, my results suggest that foliar anthocyanins in $P$. colorata do function as visual signals, however their effect on herbivory is small. Additionally, interindividual variation in non-senescing leaf colour in P. colorata may be stable due to a trade off between signalling and photosynthesis. Discussions of leaf signalling need to follow the examples of other fields studying the interactions between plants and insects and move from overly simple models to those that incorporate more of the complexity that is observed in the natural world. 


\section{ACKNOWLEDGEMENTS}

This thesis would not have been possible without the vast amount of academic and scientific advice that I have received from: Martin Schaefer, Nigel Perry, John van Klink, Barry Logan, Niky Hughes, KC Burns, Phil Lester, and George Gibbs. In particular, I would like to thank my primary supervisor Kevin Gould - I have hugely appreciated your insight, encouragement, generosity, and lightening-fast written feedback.

I have also received a phenomenal amount of practical assistance. Thank you to: Luke Youard for sharing your data, Anne Barrington for rearing countless larvae, Catherine Sansom for assistance with GC-MS, Craig Doney for fabricating Y-tubes, Frederick Wehrle for the colour-detector R-script, Loïc Cizabuiroz for help with lab trials, Kaylyn Carpenter for expertly wrangling the Li-Cor under adverse field conditions. Also, thanks to all the lovely people who helped with fieldwork: Dad, Luke Cooney, Karl Yager, Mikey Willcox, James Shannon, Daniel Wehnert and Alex Sascha Feary.

This research was funded by a Royal Society of New Zealand Marsden Grant. I also received funding from the NZ Society for Plant Biology and Victoria University of Wellington for travel to international conferences and to carry out research at Martin Schaefer's lab in Germany.

I owe many thanks to all my family for their love and support. In particular, my parents for helping so much in so many ways, and Ophelia for kidnapping me for coffees. To my darling Greta, and our children, Ari, Flynn and Pepper: you've provided an excellent distraction from thesis work! Thank you for your patience, sacrifices and tolerance of my science-trances over the last four years.

Lastly, I wish to express my gratitude to God, the creator of this unfathomably diverse and wonderful universe: Thank you for your faithfulness, and grace. 


\section{TABLE OF CONTENTS}

FRONTISPIECE

$\begin{array}{lll}\text { ABSTRACT } & \text { v }\end{array}$

ACKNOWLEDGEMENTS vii

TABLE OF CONTENTS viii

LIST OF FIGURES $\quad$ x

LIST OF TABLES X xii

1 General Introduction 1

1.1 Leaves are usually green to optimise photosynthesis 1

1.2 Variation in leaf colour 1

1.3 Anthocyanin pigment description 2

$\begin{array}{lll}1.4 & \text { Anthocyanic leaves are abundant } & 4\end{array}$

$\begin{array}{lll}\text { 1.5 Possible adaptive functions for foliar anthocyanin pigments } & 6\end{array}$

1.6 Review of evidence in support of leaf signalling hypothesis 16

$\begin{array}{lll}1.7 & \text { Alternative hypotheses for autumn leaf colouration } & 19\end{array}$

$\begin{array}{lll}1.8 & \text { Signalling in senescing autumn vs. non-senescing leaves }\end{array}$

1.9 Review of criticisms of leaf signalling hypothesis 22

$\begin{array}{lll}1.10 & \text { Gap in knowledge } & 25\end{array}$

1.11 Pseudowintera colorata is an ideal species to study leaf signalling 26

$\begin{array}{lll}1.12 & \text { Aims of the thesis } & 27\end{array}$

2 Do foliar anthocyanins function as a visual signal in $P$. colorata? 31

$\begin{array}{lll}2.1 & \text { ABSTRACT } & 31\end{array}$

$\begin{array}{lll}2.2 & \text { INTRODUCTION } & 31\end{array}$

2.3 MATERIALS AND METHODS 35

2.4 RESULTS 41

$\begin{array}{lll}2.5 & \text { DISCUSSION } & 57\end{array}$

3 Volatile profiles of red and green $P$. colorata leaves 65

$\begin{array}{lll}3.1 & \text { ABSTRACT } & 65\end{array}$

$\begin{array}{lll}3.2 & \text { INTRODUCTION } & 65\end{array}$

$\begin{array}{lll}3.3 & \text { MATERIALS AND METHODS } & 69\end{array}$

$\begin{array}{lll}3.4 & \text { RESULTS } & 77\end{array}$

$\begin{array}{llr}3.5 & \text { DISCUSSION } & 85\end{array}$

$\begin{array}{lll}3.6 & \text { CONCLUSIONS } & 90\end{array}$ 
4 Photosynthesis of red and green $P$. colorata leaves 91

$\begin{array}{lll}4.1 & \text { ABSTRACT } & 91\end{array}$

$\begin{array}{lll}4.2 & \text { INTRODUCTION } & 91\end{array}$

4.3 MATERIALS AND METHODS

$\begin{array}{lll}4.4 & \text { RESULTS } & 100\end{array}$

$\begin{array}{lll}4.5 & \text { DISCUSSION } & 108\end{array}$

5 Feeding and oviposition preference of the insect herbivores of $P$. colorata 118

$\begin{array}{lll}5.1 & \text { ABSTRACT } & 118\end{array}$

$\begin{array}{llr}5.2 & \text { INTRODUCTION } & 118\end{array}$

5.3 MATERIALS AND METHODS

$\begin{array}{llr}5.4 & \text { RESULTS } & 124\end{array}$

$\begin{array}{lll}5.5 & \text { DISCUSSION } & 128\end{array}$

6 Nutrient status of red and green Pseudowintera colorata leaves 134

$\begin{array}{lll}\text { 6.1 ABSTRACT } & 134\end{array}$

$\begin{array}{lll}6.2 & \text { INTRODUCTION } & 134\end{array}$

6.3 MATERIALS AND METHODS 136

$\begin{array}{lll}\text { 6.4 RESULTS } & 136\end{array}$

$\begin{array}{lll}6.5 & \text { DISCUSSION } & 138\end{array}$

6.6 CONCLUSIONS 139

7 General discussion $\quad 140$

$\begin{array}{lll}7.1 & \text { INTRODUCTION } & 140\end{array}$

7.2 There is strong empirical evidence in support of a leaf signalling function for foliar anthocyanins in Pseudowintera colorata. 140

7.3 In terms of herbivory, the effect of leaf signalling in P. colorata is small. 141

7.4 Alternative hypotheses cannot be ignored. 142

7.5 Inter-individual variation in non-senescing leaf colour may be stable due to a trade off between signalling and photosynthesis. 144

$\begin{array}{lll}7.6 & \text { CONCLUSIONS } & 148\end{array}$

$\begin{array}{lr}\text { Appendicies } & 149\end{array}$

A. Induced vs. natural leaf redness 149

B. Insect fitness upon diet of red or green P. colorata leaves 151

$\begin{array}{ll}\text { References } & 154\end{array}$ 


\section{LIST OF FIGURES}

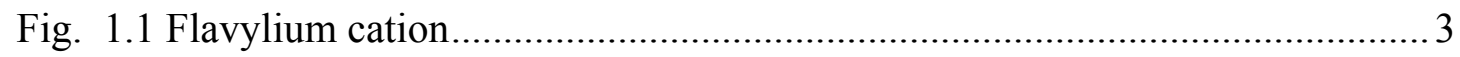

Fig. 1.2 Mean ( $\pm \mathrm{SE})$ reflectance spectra of red and green portions of the adaxial

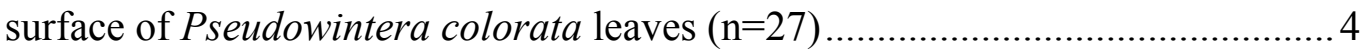

Fig. 2.1 (A) Patterns of colouration of $P$. colorata leaves; (B) a matched pair of $P$. colorata trees contrasting in colour; photographs representative of (C) Otago and (D) Wellington sites; (E) Ctenopseustis sp. leafroller caterpillar within $P$. colorata leaves; and (F) transverse section through a $P$. colorata leaf showing idioblast, red anthocyanin pigments and structure of polygodial

Fig. 2.2 Individuals' mean and coefficient of variation for $(\mathbf{A})$ leaf redness, $(\mathbf{B})$ anthocyanin and $(\mathbf{C})$ chlorogenic acid concentrations .

Fig. 2.3 Individuals' mean and coefficient of variation for (A) polygodial and (B) 9deoxymuzigadial concentrations and $(\mathbf{C})$ herbivory levels .....

Fig. 2.4 Within-individual patterns in (A) leaf colour, and (B) anthocyanin and (C) chlorogenic acid concentrations (Wellington).

Fig. 2.5 Within-individual patterns in (A) polygodial and (B) 9-deoxymuzigadial concentrations and $(\mathbf{C})$ herbivory levels (Wellington).

Fig. 2.6 Within-individual patterns in (A) leaf colour and (B) anthocyanin concentration (Otago)

Fig. 2.7 Within-individual patterns in (A) polygodial and (B) 9-deoxymuzigadial concentrations and $(\mathbf{C})$ herbivory levels (Otago)

Fig. 2.8 Proportionate leaf redness and polygodial concentrations in leaves of $P$. colorata from (A) Wellington and (B) Otago

Fig. 2.9 Feeding damage and red area of P. colorata leaves from (A) Wellington and

(B) Otago 55

Fig. 3.1 Oven bags and SPME fibres used to collect VOCs in the field 70

Fig. 3.2 (A) GC-MS autosampler; (B) Leaves and caterpillars in vials 71

Fig. 3.3 Y-tube olfactometer ..... 75

Fig. 3.4 Night time VOC profiles from red and green P. colorata in the field .......... 78

Fig. 3.5 VOC profiles of browsed and unbrowsed $P$. colorata leaves 81

Fig. 3.6 Principal component biplot showing variation in VOC profiles for red and green $P$. colorata leaves from potted plants exposed to herbivores. 
Fig. 3.7 VOC profiles from mechanically damaged leaves from red and green matched pairs of $P$. colorata trees

Fig. 3.8 Olfactory preferences of 'naïve' and 'experienced' C. obliquana larvae for mechanically damaged red- and green-coloured $P$. colorata leaves

Fig. 4.1 Transmittance spectra from Novaflex fibre optic illuminator and Li-Cor LED light sources 98

Fig. 4.2 Light response curves for $\mathrm{CO}_{2}$ assimilation of green and red P. colorata leaves in the field.

Fig. 4.3 Light saturated rate of $\mathrm{CO}_{2}$ assimilation for matched green and red $P$. colorata leaves

Fig. 4.4 Concentrations of chlorophyll $a$ pigments in matched red and green $P$. colorata leaves 103

Fig. 4.5 Light response curves for (A) photochemical quantum yield, (B) photochemical quenching, and (C) non-photochemical quenching of photosystem II for matched red and green leaves of $P$. colorata 106

Fig. $4.6 F_{\mathrm{v}} / F_{\mathrm{m}}$ of matched red and green $P$. colorata leaves before and after exposure to strong white light. 107

Fig. $4.7 F_{\mathrm{v}} / F_{\mathrm{m}}$ of matched red and green $P$. colorata leaves before and after exposure to white or red/blue light 108

Fig. 5.1 Photographs of feeding preference bioassays for caterpillars (A), weta (B), and stick insects $(\mathbf{C})$

Fig. 5.2 Feeding preferences of a variety of endemic NZ chewing invertebrates for green and red coloured $P$. colorata leaves

Fig. 6.1 Leaf nutrient content of green and red P. colorata leaves 


\section{LIST OF TABLES}

Table 2.1 Results from general linear mixed-model analyses of the relationships between the leaf redness, chemistry and herbivory from $P$. colorata trees in Wellington and Otago.

Table 2.2 Herbivore pressure and fitness parameters of matched pairs of red and green $P$. colorata trees. 56

Table 3.1 Results from MANOVA analyses of the relationships between the colour and VOC emission profiles of intact, herbivore-, and mechanically-damaged leaves from matched pairs of $P$. colorata.

Table 3.2 Results from Discriminant Function analyses of the relationships between the colour and VOC emission profiles of intact, herbivore-, and mechanicallydamaged leaves from matched pairs of $P$. colorata

Table 3.3 Discriminant function structure matrix displaying unstandardized DF coefficients for each principle component calculated from the VOC profiles of browsed and unbrowsed $P$. colorata leaves.

Table 4.1 Median and interquartile range for air temperature, relative humidity, and wind speed during sampling days.

Table 4.2 Parameters of the initial phase of $\mathrm{CO}_{2}$ assimilation light curves from matched green and red $P$. colorata leaves

Table 4.3 Concentrations of chlorophylls $a$ and $b$ and carotenoid pigments in matched pairs of red and green leaves of $P$. colorata

Table 4.4 A list of studies classified as either in support of, equivocal, or against a photoprotective function for anthocyanin pigments.

Table 5.1 List of species observed on P. colorata at two fieldsites in the lower North Island of New Zealand 125

Table 5.2 Frequency of herbivory by Ctenopseustis spp. leafrollers upon P. colorata leaves in feeding preference bioassays.

Table 5.3 Frequency of oviposition by gravid C. obliquana females upon P. colorata leaves in two types of bioassays. 


\section{General Introduction}

\subsection{Leaves are usually green to optimise photosynthesis}

Leaves are primarily photosynthetic organs. Their main function is to use light to make the sugars and energy required for plant growth and reproduction. To capture light, the photosynthetic machinery of land plants uses chlorophyll $a$ and $b$ pigments, which strongly absorb blue and red light (Nishio 2000, Lee 2007), while only weakly absorbing green light (Terashima et al. 2009). As a consequence, leaves typically appear green to our eyes.

At first glance it may appear inefficient to not strongly absorb all available light for photosynthesis. Indeed, in addition to chlorophylls $a$ and $b$, some species of green algae do use photosynthetic pigments that absorb green light, and therefore these organisms appear black (Terashima et al. 2009). However, for the leaves of land plants, it is thought that there is an adaptive benefit to being green (i.e. only weakly absorbing green light). Under non-saturating light, blue and red light, which is strongly absorbed by chlorophyll, is used for photosynthesis (Nishio 2000). Under saturating light, when the chloroplasts in the upper leaf cell layers may be operating at their maximum photosynthetic capacity, the absorption properties of chlorophyll pigments allows green light to penetrate deep into the mesophyll, where it drives photosynthesis (Sun et al. 1998, Terashima et al. 2009). Therefore, the leaves of land plants can achieve high photosynthetic efficiency under the highly variable light conditions typical of terrestrial environments (Nishio 2000, Terashima et al. 2009). However, despite the advantages to being green, much variation in leaf colour exists (Lee 2007).

\subsection{Variation in leaf colour}

\subsubsection{Different mechanisms for non-green leaf colour}

Non-green leaf colouration arises through different mechanisms. Leaves of some tropical understory species appear iridescent blue, due to the interaction of light with specialised structures within the upper surface of leaf epidermal cells (Gould and Lee 1996, Glover and Whitney 2010). White leaf colouration can be caused by a dense covering of trichomes (Lev-Yadun 2006b), or, in variegated leaves, by the absence of 
chlorophyll pigments within cells from patches of leaf tissue (Lee 2007). Silver or grey patches of some variegated leaves can be caused by the reflection of light from air spaces beneath the adaxial epidermis (Hoch et al. 1980, Sheue et al. 2012). However, the majority of non-green leaf colouration is caused by light interacting with pigment molecules within leaf cells.

Yellow and orange leaf colours typically indicate the presence of carotenoid pigments. Products of the isoprene pathway, carotenoid pigments are abundant in leaves, as they function as accessory pigments (assisting photosynthesis) and as part of the quenching mechanisms of photoprotection (Demmig-Adams and Adams III 1996, Ougham et al. 2005a, Lee 2007). Their presence is usually masked by the higher concentrations of chlorophyll pigments in non-senescing leaves (Sanger 1971). Only during leaf senescence are their colours revealed.

\subsubsection{Red Pigments}

There is a diverse range of red pigments found in terrestrial plants. Two relatively rare classes of red compounds are the thiarubrines and quinone methides (Lee 2007). In certain families of one order of plants, the Caryophyllales, betalain pigments are responsible for red and yellow colouration. In almost every other order of plants, red leaf colouration is produced by anthocyanin pigments (Lee 2002, 2007). Brown leaf colour is the most commonly the result of the presence of both chlorophyll and low concentrations of anthocyanins (Lee 2007). Anthocyanins are the most well-studied, widespread and important class of red leaf pigments (Lee 2007). Even though they have been the subject of study for over 100 years, there is still heated scientific debate surrounding the function of anthocyanin pigments within leaves.

\subsection{Anthocyanin pigment description}

The chemistry, biosynthesis and molecular genetics of anthocyanin pigments are very well understood. Anthocyanins have received more scientific investigation than any other secondary metabolic compound (Mol et al. 1996, Winkel-Shirley 2001, Lee and Gould 2002a, Saito and Yamazaki 2002, Bueno et al. 2012). This is due to their significance for human health, as well as for commerce, in particular floriculture, agriculture and as food colourants (Andersen and Jordheim 2005). 
Anthocyanin pigments are an end-product of the flavonoid biosynthetic pathway. An anthocyanin molecule consists of an anthocyanidin (based on the flavylium cation Fig. 1.1) bonded to one or more glycosides (Strack and Wray 1989). Modification of this basic structure through the substitution of hydroxyl groups, methylation, acylation, and glycosylation with different sugars at different positions results in a remarkable diversity of anthocyanins (Harborne 1967). Over 500 naturally occurring structures have been identified from plants to date (Andersen and Jordheim 2005).

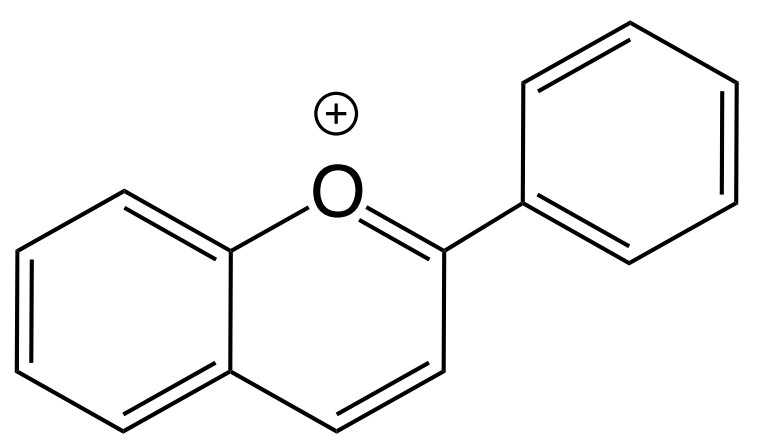

Fig. 1.1 Flavylium cation

The light absorption properties, and subsequent colour, of anthocyanin molecules depends on the specific anthocyanidin, $\mathrm{pH}$, the extent of acylation, co-pigmentation with other flavonoids, and/or chelation to metal ions (Neill 2002). These mechanisms give rise to the vibrant pinks, reds, blues and purples of many flowers and fruits. In aqueous solutions, the anthocyanidin component of anthocyanin molecules exists as a complex of several differently coloured structures. The equilibrium between these structures, and the resulting colour of the solution, changes according to $\mathrm{pH}$. They appear red in strongly acid environments $(\mathrm{pH}<3)$, colourless when slightly acidic (pH $4-5)$, blue at neutral to slightly basic conditions (pH $7-8)$, and yellow when strongly alkaline $(\mathrm{pH}>8)$ (Andersen and Jordheim 2010, Bueno et al. 2012).

Anthocyanins are synthesized in the cytoplasm as colourless forms and are quickly transported to the vacuole, although in rare occasions, anthocyanin pigments may bind to the cell wall (Neill 2002, Andersen and Jordheim 2005, Philpott et al. 2009). Vacuolar $\mathrm{pH}$ is maintained at around $\mathrm{pH}$, however inside the vacuoles of vegetative tissue of plants anthocyanins are usually stabilised as red forms (Bueno et al. 2012). These pigments absorb green light (between $495-570 \mathrm{~nm}$ ), thereby reducing the 
amount of green light reflected from the leaf surface (Fig. 1.2). Therefore, to our eyes, anthocyanic leaves appear red. In leaves, the most common anthocyanin is cyanidin-3-glucoside (Harborne 1967).

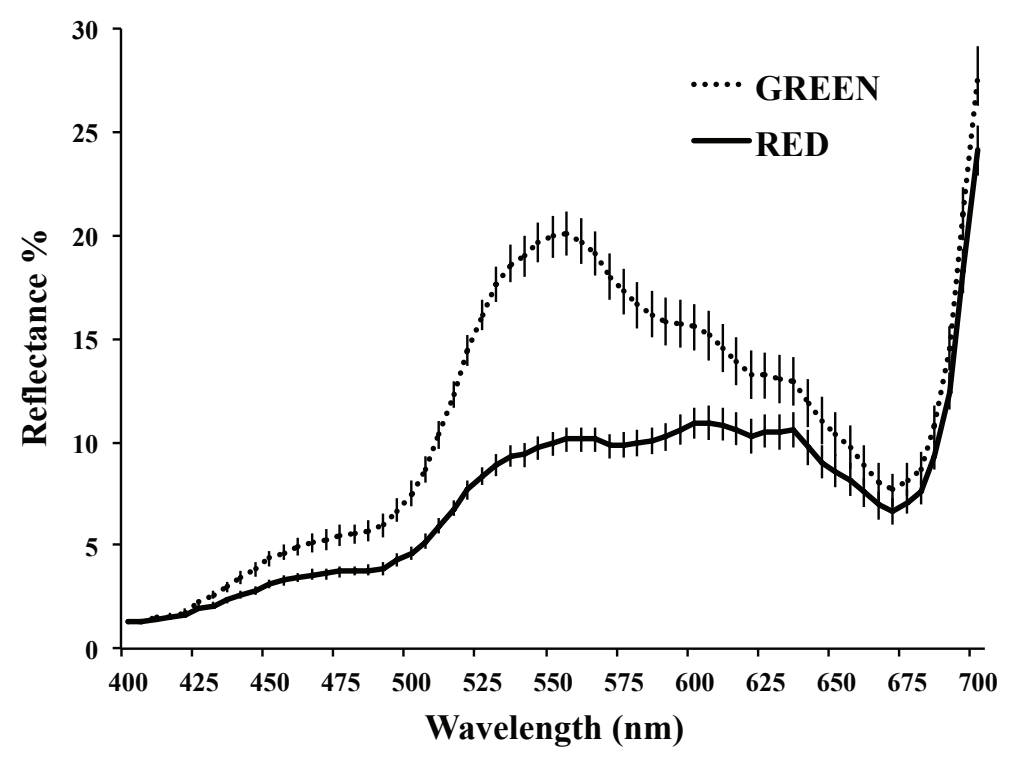

Fig. 1.2 Mean $( \pm \mathrm{SE})$ reflectance spectra of red and green portions of the adaxial surface of Pseudowintera colorata leaves $(\mathrm{n}=27)$.

\subsection{Anthocyanic leaves are abundant}

Red anthocyanic leaves are taxonomically and geographically widespread. They are produced by species throughout almost all orders of the plant kingdom and can be found in all terrestrial biomes, as well as some aquatic habitats (Lee 2002, Novak and Short 2011).

\subsubsection{Temporal and spatial patterns of anthocyanin accumulation in leaves}

The phenology of anthocyanin expression within leaf tissues varies among species and individuals. Anthocyanin pigments can be maintained within leaves throughout their lifespan (Gould et al. 2000), be restricted to a particular phase of leaf development, as with the red flushes in young expanding leaves and red colours of senescing foliage of deciduous tree species (Coley and Aide 1989, Gould 2004, Ougham et al. 2005a, Archetti 2009c), or show seasonal patterns, as with the winter reddening of evergreens (Hughes 2011). 
Additionally, the across-leaf spatial distribution of anthocyanin accumulation can vary among species and individuals. Anthocyanins may occur uniformly across the upper, lower or both leaf surfaces. Alternatively, red colouration can be localised to specific areas of leaves (stipule, petiole, veins, interveinal tissue, margins, or domatia) or patchily distributed in spots, patches or stripes over the upper, lower or both surfaces of the leaf lamina. (Wheldale 1916, Lee 2007, Wong and Srivastava 2011, Cooney et al. 2012). These patterns are achieved by the production of anthocyanin pigments in the vacuoles of different cell layers within leaves. Within the leaf lamina anthocyanins are commonly located in palisade mesophyll, but can also be produced by spongy mesophyll, and adaxial and abaxial epidermal cells (Wheldale 1916, Gould and Quinn 1999, Steyn et al. 2002, Hughes and Smith 2007b, Merzlyak et al. 2008).

\subsubsection{Interspecies variation in foliar anthocyanins}

There are differences in colouration among species. Many plant species do not produce anthocyanic leaves. Of these, many plants produce anthocyanins at other locations (e.g. hypocotyls, fruits) but not in leaves. The species that do produce foliar anthocyanins vary in their spatial patterns of anthocyanin accumulation (see section 1.4.1). Interspecies variation in autumn senescing leaf colouration has drawn the attention of several researchers (Matile 2000, Hamilton and Brown 2001)

There appears to be large-scale variation in the interspecies patterns of anthocyanic leaf colouration in autumn senescing leaves. From a description of autumn leaf colouration of 2368 tree species compiled by Archetti (2009c), Lev-Yadun and Holopainen (2009) compared autumn leaf colouration of tree species found in northern Europe, North America and East Asia. They found that anthocyanic senescing leaves are more common among species from North America and East Asia, than in northern Europe (where autumn senescing foliage is predominantly yellow).

\subsubsection{Intraspecies variation in foliar anthocyanins}

Patterns of anthocyanin accumulation also vary within species, even among neighbouring individuals. While it is hard to classify the colour of whole individual plants, individuals may differ in the mean intensity of red colouration, or in the degree of redness (proportion of leaves coloured red), or in the specific pattern of red colouration. Intraspecific heterogeneity in red pigmentation has been described 
among individuals with: juvenile flushing leaves in tropical (Lee and Gould 2002b) and temperate deciduous species (Taulavuori et al. 2011); leaves that are red only in winter (Kytridis et al. 2008, Hughes 2011), leaves where red colouration is maintained year round (Gould et al. 2002a, Neill et al. 2002b, a, Wong and Srivastava 2011), senescing leaves that are red in autumn (Hamilton and Brown 2001, Archetti and Leather 2005, Rolshausen and Schaefer 2007, Karageorgou et al. 2008) and among the leaves of seagrass individuals (Novak and Short 2010).

\subsection{Possible adaptive functions for foliar anthocyanin pigments}

Observations of the patterns of foliar anthocyanin accumulation in nature, combined with experimental manipulation, have revealed that anthocyanin biosynthesis is inducible. Anthocyanins biosynthesis in leaves can be induced by a range of abiotic and biotic stressors, including: cold, high light, UV radiation, heavy metal toxicity, mechanical stress, as well as fungal and herbivore damage (Chalker-Scott 1999, Gould et al. 2002a). The immense variability in the patterns of anthocyanin accumulation has lead to a diverse range of hypotheses being developed to explain the function of these pigments within leaves (Gould 2004, Archetti 2009a, Hughes 2011). These hypotheses can be classified into those proposing an abiotic or a biotic function for foliar anthocyanins. Below, I have briefly described each hypothesis. I have highlighted the best-substantiated hypothesis within each class, according to their relative theoretical and empirical support.

\subsubsection{Abiotic hypotheses for foliar anthocyanin pigments}

Many of the proposed functions for foliar anthocyanins pigments have not been supported by empirical and theoretical investigations.

\subsubsection{Leaf warming}

Pigments absorb light energy (Neill and Gould 1999). It has been hypothesised that the role of anthocyanin pigments in abaxial surfaces of leaves in the tropical understory is to absorb light energy and therefore increase leaf temperature, and aid transpiration in damp tropical understory environments (Wheldale 1916). The assumption of this hypothesis, regarding light absorption, is sound. Intraspecific comparisons of red and green leaves at similar developmental stages consistently show that red leaves do absorb up to $17 \%$ more light energy than similar green leaves. This is true for winter-reddening species (Hughes et al. 2005), year-round 
polymorphic species (Neill and Gould 1999) and autumn senescing species (Merzlyak et al. 2008).

Early measurements of leaf temperature supported this hypothesis (Smith 1909). However, subsequent measurements by Lee et al. $(1979,1987)$ with more sensitive equipment were not consistent with a warming function for anthocyanins. When comparing the temperatures of anthocyanic juvenile with green mature leaves of 6 species from tropical understory habitats, the juvenile leaves of only one species showed a slight increase $\left(0.5^{\circ} \mathrm{C}\right)$ in temperature and this difference disappeared on sunny days. When testing if this hypothesis applies to red autumn colouration, Lee et al. (2003) found no temperature difference between green and senescing red leaves of two species. Therefore, it seems that leaf warming, if it occurs at all, is not a universal explanation for the presence of foliar anthocyanins.

\subsubsection{Backscatter hypothesis}

Lee et al. (1979) hypothesised that the function of abaxial anthocyanin pigments is to internally reflect transmitted red light back to chloroplasts in the mesophyll, so that it may be used for photosynthesis. This was thought to provide an advantage in the low light conditions of tropical understory environments. However, Gould et al. (1995) found no differences in the abaxial reflectance between red and green leaves of two tropical species. Hughes et al. (2008) looked at light absorbance within abaxially red/green variegated leaves of Begonia heracleifolia. In contrast to the predictions of the backscatter hypothesis, abaxial green leaf surfaces were more reflective than those with anthocyanins, and under identical illumination there was no difference in chlorophyll fluorescence (a measure of photosynthesis) between green and red portions of the leaf.

\subsubsection{Osmotic adjustment}

Several strands of evidence point towards an osmotic function for foliar anthocyanins. The biosynthesis of anthocyanin pigments can be induced in cell cultures and whole plant systems under experimental and natural drought conditions. Species that produce anthocyanin pigments in their leaves are more common in environments with low soil moisture (Chalker-Scott 1999). Aside from drought, other environmental stressors such as ozone, heat and cold temperatures, wind, anoxia (caused by flooding), nutrient deficiency, metal toxicity, and salinity can directly or indirectly increase the osmotic potential between the cell and interstitial spaces, causing water 
loss and plasmolysis. Additionally, anthocyanins are commonly produced at times when osmotic stress is more likely, e.g. in the damp tropical understory, the developing tissues of flushing leaves may face difficulty maintaining turgor pressure vital for leaf expansion. Similarly, in temperate climates, reddening of developing and senescing leaves coincides with the risk from late winter and early autumn frosts respectively (Chalker-Scott 2002). These observations led to the hypothesis that anthocyanin pigments function as solutes, decreasing leaf cell osmotic potential to help plants tolerate sub-optimal conditions (Chalker-Scott 2002). Manetas (2006) questioned the suitability of anthocyanins due to their low concentrations relative to other solutes in leaves. In a comparison of species to see if drought tolerance through osmotic adjustment explains the production of anthocyanins using winter red evergreens, Hughes et al. (2010a) found that winter reddening species were more likely to exhibit physiological features characteristic of drought tolerance and acclimation (i.e., a more negative midday osmotic potential, more negative osmotic potential at full turgor, and greater cell wall thickening) than green-leafed species during winter. However, variability in the data indicated that an osmoregulatory function for anthocyanins is not sufficient to explain winter colour change in angiosperm evergreens. Recent research has confirmed Manetas's (2006) speculation; due to their relatively low concentrations in leaves, anthocyanin pigments represent a very small contribution to osmotic adjustment during drought acclimation of winterreddening evergreens (Hughes et al. 2012b).

\subsubsection{Ultraviolet (UV) radiation screen}

UV-B radiation can be damaging to DNA (Hoque and Remus 1999). It has been well documented that, in many plants, anthocyanin biosynthesis is induced by exposure to high UV (Chalker-Scott 1999, Gould 2004). Consequently anthocyanin pigments within leaves have been hypothesised to function as a UV-B screen (Lee and Lowry 1980). In support of this, Stapleton and Walbot (1994) associated the presence of anthocyanins with reduced DNA damage in Zea mays plants under high UV-B treatments. However, there is now a widely held view that UV protection is not an important function of foliar anthocyanin pigments (Gould 2004). Most commonly found in mesophyll, the location of anthocyanins within leaves is suboptimal to act as a UV screen (Wheldale 1916, Gould and Quinn 1999, Hatier and Gould 2008a). Other colourless flavonoids (located in epidermis) more strongly absorb UV than the anthocyanins typically found in leaves (Woodall and Stewart 1998). Finally, in rice 
(Oryza sativa), the mechanisms to repair UV-B-damaged DNA are triggered by a combination of blue and UV-A light. Under high UV conditions, purple anthocyanic varieties exhibited reduced growth, presumably due to absorption of blue light by anthocyanins, inhibiting DNA repair (Hada et al. 2003).

\subsubsection{Photoprotection hypothesis}

As the primary function of leaves is photosynthesis, the most widely accepted explanation for the presence of anthocyanins in leaves is protection of photosynthetic machinery. Many plants regularly experience an oversupply of light (Long et al. 1994, Alves et al. 2002, Wilhelm and Selmar 2010). Excess light energy can inhibit photosynthesis and cause damage to the thylakoid membranes in the chloroplast, and injure the surrounding cell and leaf tissues (Adir et al. 2003, Demmig-Adams and Adams III 2003, Adams III et al. 2006, Demmig-Adams and Adams III 2006). Several properties of foliar anthocyanins make them appropriate as photoprotectants.

\subsubsection{Light screen}

To reduce damage caused by light stress, anthocyanin pigments in leaves may function as a light screen, attenuating incident light energy. In acidic solutions, anthocyanin pigments have a high molar extinction coefficient, and very effectively absorb green light (500 - $600 \mathrm{~nm}$ ) even when present at very low concentrations (Neill and Gould 2003). In leaves, anthocyanin pigments have been shown to absorb green light that otherwise might be used for photosynthesis of lower cell layers (Pietrini and Massacci 1998, Neill and Gould 1999, Nishio 2000, Feild et al. 2001, Gould et al. 2002b, Hughes et al. 2005, Karageorgou and Manetas 2006, Hughes and Smith 2007a), but see van den Berg (2009) who found different results for juvenile and senescing Acer saccharum leaves. As further evidence of light attenuation by anthocyanin pigments, red leaves from exposed locations frequently exhibit the morphological and physiological characteristics of shade-acclimated leaves (Boardman 1977, Givnish 1988, Gould et al. 2002b, Manetas et al. 2003, Hughes and Smith 2007b, Kyparissis et al. 2007, Zeliou et al. 2009, Nikiforou and Manetas 2010, Nielsen and Simonsen 2011, Nikiforou et al. 2011, Zhang et al. 2011).

\subsubsection{Antioxidant}

Many 'normal' cell processes, such as photosynthesis and respiration, cause the formation of low levels of harmful reactive oxygen intermediates (ROIs)(Mittler 2002). In low quantities, ROIs are not thought to be deleterious, and in fact, may 
serve important roles in cell signalling processes (Mittler 2002). However, stressors (such as excess light, cold temperatures, drought stress, salt stress, heavy metal toxicity, UV radiation, air pollution, mechanical stress, nutrient stress, pathogen attack and wounding) can result in the formation of much higher levels of ROI leading to oxidative damage of cell components and programmed cell death (Demmig-Adams and Adams III 1992, Mittler 2002, Demmig-Adams and Adams III 2006). Because anthocyanin biosynthesis is often induced in response to these same stressors, it has been hypothesised that foliar anthocyanins function as antioxidants, scavenging excess ROI. This has lead to anthocyanin pigments being the subject of intense study with regards to human health (He and Giusti 2010).

Certain anthocyanin pigments have been demonstrated to be powerful antioxidants in vitro, having antioxidant capacities up to four times greater than those of vitamin $\mathrm{E}$ and C analogues (Rice-Evans et al. 1997, Wang et al. 1997, Neill and Gould 2003, Gould 2004). Several studies indicate that the antioxidant property of anthocyanin is biologically important in leaves. For example, Neill et al. (2002a) demonstrated that anthocyanins were responsible for red leaves of Elatostema rugosum having a higher ROI-scavenging capacity relative to acyanic leaves. Gould et al. (2002a) showed that red areas of Pseudowintera colorata leaves more rapidly scavenged $\mathrm{H}_{2} \mathrm{O}_{2}$ produced by mechanical wounding, than green areas. In addition, the ROI scavenging potential of leaves of the Arabidopsis (tt3tt4) mutant, unable to synthesise anthocyanins, decreased during prolonged exposure to high light stress, while the antioxidant capacities of the wildtype were maintained (Zhang et al. 2012). However, anthocyanin pigments in red leaves do not always provide an antioxidant advantage relative to acyanic leaves (Neill et al. 2002b, van den Berg and Perkins 2007, Hughes et al. 2012a).

The cellular and histological location of anthocyanin pigments relative to the source of free radicals is important. Within cells, free radicals are mostly produced by the cell walls and cytoplasmic organelles, therefore (with the possible exception of $\mathrm{H}_{2} \mathrm{O}_{2}$ which rapidly diffuses through the tonoplast) vacuolar anthocyanins are not optimally located to intercept them. Stronger antioxidants than anthocyanins (such as superoxide dismutase, ascorbate peroxidase and catalase) do occur in the cytoplasm, chloroplasts and other organelles, closer to the sites of ROI production (Mittler 2002). 
With regards to histology, leaves with anthocyanins in the mesophyll (where chloroplasts are predominantly located) show higher antioxidant capacity than leaves where anthocyanins are located in epidermal cells (further from the site of photoinhibition) (Neill and Gould 2003, Kytridis and Manetas 2006). Anthocyanins' antioxidant properties are likely to be of functional significance. However, given the variability in the patterns of anthocyanin colouration of leaves (Lee and Collins 2001), the relative importance of an antioxidant function for anthocyanins is likely to vary between- and within-species (Hatier and Gould 2008a).

\subsubsection{Modulation of cellular signalling cascades}

Increasingly ROI are recognised to play a signalling role within cells (Mittler 2002). ROI are actively produced at low levels by plant cells as part of normal healthy functioning, acting as signals that trigger changes in cell physiology, metabolism and gene expression (Foyer and Noctor 2005). Antioxidant enzymes maintain cellular ROI levels at the low levels needed for cellular signalling, however high levels of ROI can cause oxidative damage and lead to programmed cell death. Hatier and Gould (2008b, a) proposed that anthocyanin pigments may assist in regulating ROI and subsequent responses to stress through three mechanisms: a) reducing ROI production by attenuating light; b) directly scavenging ROI; and, c) directly participating in cellular signalling pathways. This hypothesis lacks direct empirical evidence.

\subsubsection{Evidence for a photoprotective function for foliar anthocyanins}

The most widely accepted physiological function for foliar anthocyanins in leaves is photoprotection by both absorbing ROI and attenuating high energy quanta surplus to the requirements for photosynthesis, as well as possibly through interactions with cellular signalling pathways (Hatier and Gould 2008a). It is difficult to gauge the relative contribution of the light screening and antioxidant properties of anthocyanins in planta (Neill and Gould 2003). Nevertheless, there is strong empirical evidence that foliar anthocyanins protect from photooxidative stress (summarised in Table 4.4). Following exposure to saturating light flux, red leaves tend to be less photoinhibited and/or recover more quickly than comparable green leaves (Feild et al. 2001, Manetas et al. 2003, Neill and Gould 2003, Hughes et al. 2005, Gould et al. 2010, Nielsen and Simonsen 2011). Additionally, photoprotection by foliar anthocyanins in autumnsenescing leaves has been shown to increase beneficial nutrient resorption before leaf fall (Hoch et al. 2001, Hoch et al. 2003). There is an impressive amount of evidence 
in support of this hypothesis, however several studies have reported no photoprotective advantage for foliar anthocyanins, and there are some examples of red leaves being more susceptible to light stress than green. While, these discrepancies are not well understood, these results indicate that photoprotection is not the unifying function for anthocyanins in leaves.

Among the diverse range of physiological hypotheses for foliar anthocyanins, photoprotection is the most compelling due to its applicability to a wide-range of colouration patterns. Photoprotection is a viable explanation for induced leaf redness caused by stressors that impair photosynthetic capacity, as well as potentially explaining the different phenologies of anthocyanin production: a) transient juvenile redness during leaf expansion, before the full complement of chlorophyll and maximum photosynthetic capacity has developed (Dodd et al. 1998, Manetas et al. 2002, Choinski Jr et al. 2003, Hughes et al. 2007); b) autumn senescing leaves, when temperatures are cooler and photosynthetic machinery is being degraded (Hoch et al. 2001, Holopainen and Peltonen 2002, Hoch et al. 2003, Lee et al. 2003, Ougham et al. 2005a); and, c) winter reddening in certain evergreen species (Kytridis et al. 2008, Zeliou et al. 2009, Nikiforou et al. 2010, Nikiforou et al. 2011).

\subsubsection{Biotic hypotheses for foliar anthocyanins}

In addition to being induced by abiotic stressors (see section 1.5 above), biosynthesis of anthocyanins and subsequent leaf reddening is commonly induced by leaf damage caused by herbivory (Gould et al. 2002a). Together, plants and the insects that feed on them represent half of all the described species on Earth (Schoonhoven et al. 2005, Futuyma and Agrawal 2009). It has been claimed that most frequent and important interactions among species occur between plants and herbivorous insects (Schaefer and Rolshausen 2006, Hare 2012). Additionally, besides herbivores, anthocyanins in flowers and fruit play an important role in facilitating mutualistic interactions among plants and their pollinators and fruit dispersers (Schaefer and Ruxton 2011). Accordingly, a diverse range of hypotheses for a biotic function for foliar anthocyanins have been proposed.

\subsubsection{Fruit flag hypothesis}

In some species of deciduous trees with bird-dispersed fruits, fruit ripening coincides with leaf colour change, and this colour change occurs early, before other deciduous 
species (Stiles 1982). Here foliar anthocyanin pigments are hypothesised to function as a 'fruit flag' to attract migrating birds to disperse fruits. The proposed mechanism has received experimental support. Burns and Dalen (2002) demonstrated that black fruits are slightly more apparent to birds (as estimated by removal rates of experimental fruits) against red/orange autumn foliage than against green background. Additionally, artificial red 'flags' increased fruit removal in Rhus glabra, a species with dark red fleshy fruits and red autumn-senescing foliage (Facelli 1993). However, a subsequent study concluded that asynchrony in the phenology of fruiting, leaf colour change, bird migration and fruit dispersal in $R$. glabra was not consistent with the fruit flag hypothesis. In particular, the persistence of the majority of $R$. glabra fruits beyond autumn indicated that early leaf colour change in this species is not related to peaks in fruit removal and bird abundance ( $\mathrm{Li}$ et al. 1999). This hypothesis can only apply to tree species with bird dispersed fruits where fruit ripening is synchronised with early autumnal leaf colour change and bird migration, which represent a very small proportion of deciduous trees species with anthocyanic leaves (Willson and Hoppes 1986, Hoch et al. 2001). Therefore, the fruit flag hypothesis has a specific focus and can only explain a very limited range of the observed patterns of foliar anthocyanins.

\subsubsection{Direct defence}

It has been hypothesised that anthocyanin pigments may act as a direct defence to deter harmful organisms (Coley and Aide 1989). Anthocyanin pigments have been shown to reduce fungal growth in grapes and upon media containing biologically relevant concentrations of anthocyanin pigments (Schaefer et al. 2008). However, while floral anthocyanins slowed the development of two species of lepidopteran larvae (Johnson et al. 2008), the results of a large number of studies indicate that foliar anthocyanins are not toxic to insect herbivores (Close and Beadle 2003, Schaefer and Rolshausen 2006). Schaefer and Rolshausen (2006) argued that other compounds in the flavonoid biosynthetic pathway (e.g. tannins) are far more effective defences against insect herbivores than anthocyanins.

\subsubsection{Camouflage}

By absorbing visible light, anthocyanin pigments change the colour of leaves. Nongreen colouration has been proposed to function as a camouflage, making leaves less apparent to visually-oriented herbivores. First mentioned by Stone (1979) with reference to dull brown colouration of some understory ferns in the Malaysian 
rainforest, a camouflage/crypsis function for foliar anthocyanins has been widely accepted and echoed (Juniper 1993, Dominy et al. 2002, Karageorgou and Manetas 2006, Lev-Yadun 2006a, Karageorgou et al. 2008, Lev-Yadun and Gould 2009). The hypothesis has been extended to include mottled patterns of leaf colouration (Smith 1986, Givnish 1990, Allen and Knill 1991) and the dense layer of trichomes sometime found on plants inhabiting sandy coastal or dune environments (Lev-Yadun 2006b). However, the core prediction of this hypothesis - that non-green leaf colour reduces visual apparency - has only very recently been tested experimentally. In three examples where plant foliage has been found to likely be almost undetectable to herbivores (Fadzly et al. 2009, Klooster et al. 2009, Fadzly and Burns 2010), the colours involved were dull browns and greys, mimicking dead foliage or leaf litter, rather than vivid red. There have been no specific tests of this hypothesis using red leaves.

\subsubsection{Anti-camouflage}

Lev-Yadun et al. (2004) noted that many insect herbivores are coloured green, presumably to make them cryptic against a leafy green background. The authors proposed that red and other non-green leaf colouration may allow a plant to reduce its herbivore load by subverting the camouflage of insect herbivores, making them more visually apparent to predators and parasitoids (Lev-Yadun et al. 2004). Contrary to this hypothesis, modelling by Schaefer and Rolshausen (2006) demonstrated that a more complex background may provide increased potential hiding places for a diversity of differently coloured herbivores. To my knowledge, there have been no empirical studies assessing predation rates of green herbivores on non-green leaves.

\subsubsection{Signalling hypothesis for autumn leaf colouration}

Inter-individual variability in leaf colouration (see section 1.4.3 above) is attributable to both environmental and genetic factors (Gould and Quinn 1999). This is most clearly demonstrated where neighbouring individuals express different patterns in leaf colouration while experiencing very similar environmental regimes. The maintenance of the genetic variability is not easily explained by the photoprotection function of anthocyanins (Sinkkonen et al. 2012), nor any of the other physiological or biotic hypotheses above. However, there is one hypothesis specifically developed to explain the maintenance of inter-individual variability in leaf colouration. 
The signalling hypothesis for autumn leaf colouration states that variability in autumn colouration is the result of a process of coevolution between plants and herbivorous insects (Archetti 2000, Hamilton and Brown 2001). Autumn leaf colouration coincides with the timing of migratory flights of the winged female life stages of some aphid species. The aphids take flight to select new plant hosts upon which to lay their eggs. These eggs over-winter and the emerging aphids rapidly reproduce the following spring and summer. As trees vary in their suitability as hosts, presumably there is a strong selective pressure for winged aphid females to choose the most suitable tree to lay her eggs on. Similarly as the next season's aphids would consume the tree's resources (that could otherwise be put towards growth and reproduction) one would expect there to be a strong selective pressure for trees to successfully deter mobile female aphids from landing on them. In these circumstances the emergence of a trait that increases the ability of aphids to discern the suitability of trees as hosts, will be mutually advantageous to both the aphids that perceive and respond to the trait and the tree individuals bearing that trait.

W. D. Hamilton (Archetti 2000, Hamilton and Brown 2001) proposed that bright nongreen leaf colouration is such a trait. The hypothesis states that autumn leaf colouration functions as a visual signal of plant defence - the earlier and more intense a tree's autumn leaf display, relative to its neighbouring trees, would reliably indicate its defensive commitment against insect herbivores. The authors made simultaneous inter- and intra-specific predictions, saying that signalling to insect pests explains variation in autumn leaf colouration between species, as well as between neighbouring conspecifics (Hamilton and Brown 2001). However, almost all subsequent studies have focussed upon testing the intraspecific predictions of this hypothesis. The intraspecific component of this hypothesis is central to my thesis topic; therefore I will elaborate on it further below.

\subsubsection{Communication theory - signals vs. cues}

Communication occurs when a trait of one individual (the sender) is perceived by another individual (the receiver), prompting a change in the behaviour of the receiver (Schaefer and Ruxton 2011). In particular, signalling (a specific category of communication) occurs when the perception of the sender's trait by the receiver, and the subsequent change in behaviour, results in a mutual fitness benefit for both sender and receiver (Maynard Smith and Harper 1995). In such a scenario, both the sender's 
trait and the receiver's ability to perceive and respond to the trait are maintained through natural selection - driving reciprocal coevolutionary change in the sender and receiver species.

To qualify as a 'signal', red leaf colour must be the result of mutually beneficial coevolution between a plant and herbivore. The perception of the red leaf signal must provide an advantage to both participating plant and herbivore individuals. The plant would benefit from a reduced herbivore load, and the insect pest would benefit from information allowing the selection of the least defended host trees (Maynard Smith and Harper 2003, Scott-Phillips 2008, Allison and Hare 2009). If the perception of leaf colour by the insect does not provide a fitness benefit to the plant, then it is said that the insect is responding to the colouration as a 'cue' (Otte 1974, Allison and Hare 2009). If this is the case, the adaptive function of leaf colour is not signalling.

\subsection{Review of evidence in support of leaf signalling hypothesis}

Hamilton and Brown's (2001) leaf signalling hypothesis states that bright leaf colouration is a visual signal of a tree's defensive commitment, and functions to deter approaching insect herbivores. Despite many papers emphasising the importance of empirical tests for testing this hypothesis (Atkinson 2001, Wilkinson et al. 2002, Schaefer and Wilkinson 2004) - the majority of publications citing Hamilton and Brown's (2001) paper consist of further hypothesising, theoretical studies and reviews. Below, I present the intraspecific predictions of the signalling hypothesis, and available evidence.

Intraspecific leaf signalling hypothesis predictions:

1. Early autumn leaf colour change and 'bright' non-green colouration is a signal of a tree's high defensive commitment, relative to conspecifics.

2. Upon perceiving the leaf colour signal, insect herbivores should alter their behaviour accordingly, selecting less well-defended host trees.

3. Signalling increases the fitness of participating tree and herbivore individuals (Archetti et al. 2009). 
This hypothesis has been highly controversial and has attracted heated scientific debate. To assess the appropriateness of the assumptions, as well as to understand some of the criticism of this leaf signalling hypothesis, I present available data from relevant studies below:

\subsubsection{Prediction 1.}

"Early autumn leaf colour change and 'bright' non-green colouration is a signal of a tree's high defensive commitment, relative to conspecifics"

The majority of studies testing this first prediction have relied upon indirect measures of defence. Rolshausen and Schaefer (2007) detected no relationship between leaf fluctuating asymmetry and the timing or extent of red autumn colouration in the leaves in mountain ash (Sorbus aucuparia). Several studies have found correlations between red colouration and the total phenolic content of leaves and interpreted these as evidence in support of leaf signalling (Karageorgou and Manetas 2006, Karageorgou et al. 2008, Hughes et al. 2010b). However, as only a small proportion of the total phenolic pool is known to be involved in defence (Lawler et al. 1998, Lawler et al. 1999), this measure is not a necessarily an accurate predictor of defensive commitment. Only one study to date has correlated non-green leaf colour with quantified levels of known strong insect defence compound. Cooney et al. (2012) found that leaves of Pseudowintera colorata with wider red margins contained higher levels of polygodial, a sesquiterpene dialdehyde with strong antifeedant properties.

\subsubsection{Prediction 2.}

"Upon perceiving the leaf colour signal, insect herbivores should alter their behaviour accordingly, selecting less well-defended host trees"

A variety of field studies have found correlations between bright non-green leaf colour and reduced numbers of, and/or damage from, insect herbivores (Hagen et al. 2003, Archetti and Leather 2005, Karageorgou and Manetas 2006, Rolshausen and Schaefer 2007, Ramirez et al. 2008, Wong and Srivastava 2011, Cooney et al. 2012, Markwick et al. 2012). In the majority of these studies, it was unknown if the herbivore was responding to the leaf colour signal or some other correlated trait, such as leaf volatile organic compounds (VOC) (Ougham et al. 2005a). 
Schaefer and Rolshausen (2007a) studied the influence of leaf colour on aphid settling patterns in the field by manipulating leaf colour and applying glue to branches and whole individuals of mountain ash (Sorbus aucuparia) in a natural setting. Aphids colonised trees non-randomly, however there was no difference in the number of aphids caught on differently coloured branches and individuals. The authors concluded that, although leaf colour was not important, the aphids did use cues in precontact host selection.

Cooney et al. (2012) demonstrated that visual cues mediate feeding decisions in a non-deciduous plant-herbivore system. During feeding trials with larval Ctenopseustis obliquana (Lepidoptera: Tortricidae) under manipulated light conditions, the herbivores consumed greater leaf area from green- than red-margined leaves only when lighting conditions allowed discrimination of the leaf colour. Similar results were found by Markwick et al. (2012) when comparing feeding preferences of a related tortricid caterpillar under light and dark conditions.

\subsubsection{Prediction 3.}

"Signalling increases the fitness of participating tree and herbivore individuals" In order to distinguish whether a trait is indeed a signal, data about the fitness of both participants in the putative signalling system is required. In the only study to directly assess fitness consequences of signalling, Ramirez et al. (2008) measured aphid growth rates in spring upon trees that displayed different leaf colouration the previous autumn. No correlation was detected between host autumn leaf colour and aphid reproductive rate the following spring. Several studies have collected information about the flower and fruit production of trees, however these data were collected with the purpose of assessing how the reproductive investment of the tree affects either leaf colouration (Sinkkonen 2006b, Rolshausen and Schaefer 2007) or insect behaviour (Hagen et al. 2003, Schaefer and Rolshausen 2007a), rather than testing the above prediction regarding fitness consequences for trees. Signalling theory requires that, on average, both the sender and receiver individuals receive a mutual fitness benefit. No leaf signalling study has collected direct or indirect fitness data of both plants and insects from the same system. 


\subsection{Alternative hypotheses for autumn leaf colouration}

The publication of Hamilton and Brown's (2001) leaf signalling hypothesis prompted a large number of subsequent publications presenting modifications and alternative hypothesis regarding autumn leaves. As explained below (see section 1.8), my own research will focus on non-senescing leaves, and therefore will not be directly addressing these autumn senescing leaf hypotheses. However, I have reviewed these hypotheses here, as they are instructive of both the diversity of theorising upon this subject and the wide range of connected components of plant-insect herbivore interactions.

\subsubsection{Reproductive insurance hypothesis}

In a study of autumn leaf colour change in mountain birch (Betula pubescens sp. czerepanovii), Sinkkonen (2006b) observed that trees bearing the greater number of female catkins displayed the earliest yellow leaf colouration. Subsequently, Sinkkonen (2006a) proposed the 'reproductive insurance' hypothesis, stating that the early yellow leaf colours of reproductively active trees may function as a coevolved signal, to communicate their reproductive commitment to migrating aphids. The hypothesis assumes that trees investing a large amount of resources to reproduction will be poor quality hosts for aphids. This hypothetical scenario benefits both aphids, who would be able to avoid trees that will subsequently become poor quality hosts, and signalling trees, who would experience fewer aphids feeding on nutrients that could otherwise be used for reproduction. However, contrary to the predictions of the reproductive insurance hypothesis, Hagen et al. (2003) found a positive correlation between number of catkins and leaf damage in mountain birch (Betula pubescens). Similarly, Schaefer and Rolshausen (2007a) found a strong positive correlation between fruit production and number of aphid numbers upon mountain ash (Sorbus aucuparia).

\subsubsection{Autumn leaf colour as a cue for nutrient retranslocation}

The supporting evidence presented in Hamilton and Brown's (2001) article on leaf signalling was the observation that species of aphids which make host-selecting migratory flights in autumn are more commonly associated with tree species that display bright red or yellow autumn colouration than with tree species for which the leaves remain green. Holopainen and Peltonen (2002) expanded this data set, presenting data of aphid host association and tree colour consistent with Hamilton and Brown's (2001) interspecific result, for aphid and tree species found within Finland. 
However the authors postulated an alternative mechanism to explain the observed pattern: the 'nutrient retranslocation' hypothesis states that bright red and yellow leaf colours function for photoprotection during leaf senescence and resulting retranslocation of nutrients. Consequently red and yellow leaf colour is a reliable cue for the availability of mobile nutrients. The ability to perceive where nutrient retranslocation is occurring would provide a fitness benefit for female migrating aphids, as they would be able to select hosts according to the availability of high quality food and therefore have more resources for reproduction. Holopainen and Peltonen (2002) argued that the correlation between the bright senescing foliage and aphid species demonstrates that host-alternating aphids have adapted to life upon species where nutrient retranslocation is revealed by leaf colour.

Game-theoretical modelling by Archetti (2007), demonstrated that the nutrient retranslocation hypothesis is an evolutionary possibility. However, studies into the role of colour in aphid host location reveal that, while yellow colours are more attractive than green to aphids, red colours are less attractive than green (Ramirez et al. 2008, Döring et al. 2009). Therefore, the nutrient retranslocation hypothesis is likely to apply to yellow leaf colouration only.

\subsubsection{Tritrophic signalling via volatile organic compounds}

The signalling hypothesis provides an adaptive explanation for non-green leaf colouration. In contrast, Holopainen (2008) proposed an hypothesis to explain why some deciduous trees maintain green colouration during autumn. Holopainen (2008) argued that it is expensive to remain green up to leaf abscission, as it precludes resorption of nutrients from those leaves. Therefore the adaptive reason for staying green would be to continue to be able to producing metabolically expensive VOC that attract predators and parasitoids of aphids. The cost of lost nutrients is balanced by reduced herbivory. To aphids, senescing leaves (unable to produce metabolically expensive VOC) would represent enemy-free space and high quality food due to mobilisation of nutrients. This hypothesis predicts a positive correlation between aphids and senescing red and yellow foliage.

In an empirical test of this hypothesis, Holopainen et al. (2010) compared the volatiles released from green and yellow (not red anthocyanic) leaves of Betula pendula throughout senescence. Consistent with the hypothesis, costly photosynthesis related 
VOC were released by green leaves but not yellow. This confirms the assumption that green foliage is more capable of facilitating induced indirect defense against herbivores than yellow. I am aware of no studies investigating VOC released from either senescing or non-senescing anthocyanic leaves.

\subsubsection{Attractive signal to promote tritrophic interactions}

Yamazaki (2008a) noted three observations that the leaf signalling hypothesis for autumn leaf colour failed to address: a) healthy vigorous trees may allocate resources to growth rather than defence; b) aphids may vary in their reponses to leaf colour and defences; and, c) tritrophic interations are important forces structuring herbivore communities. He therefore proposed a modification to the leaf signalling hypothesis: Rather than being a warning signal of defence, bright autumn leaves are a coevolved attractive signal - revealing which tree individuals will allocate resources to spring growth. In response myremecophilous specialist aphids, coevolved to perceive and respond to this signal, preferentially colonise signalling trees. In turn high numbers of myremecophilous aphids on signalling trees attracts aphid-tending ant species (Yamazaki 2008a).

In this hypothetical situation the trees would benefit, because aphid-tending ants would reduce the diversity and abundance of non-aphid insect herbivores and manage levels of myremecophilous aphid populations. Responding to the signal would benefit aphids two-fold: 1) they might colonise trees that allocate resources to growth in spring, ensuring a good food supply for aphid offspring; and 2) aphid-tending ants would reduce competition with other insect herbivores. Contrary to the leaf signalling hypothesis, this hypothesis predicts that aphids are attracted to bright leaf colouration (but doesn't specify red or yellow).

Myremecophilous aphids are a minority among aphids, however this hypothesis was later extended to include other hemipterans that produce honeydew (scale insects and leafhoppers) and therefore attract ants (Yamazaki 2008b). Due to the tri-trophic interactions, this hypothesis is inherently more complex than the leaf signalling hypothesis. There have been no empirical tests to date.

\subsubsection{Signalling to alert insects that leaves are about to be shed}

Lev-Yadun and Gould (2007) proposed another modification for the leaf signalling hypothesis: that autumn leaf colour may function as a signal to herbivores that leaves 
are about to be shed, rather than signal of plant defensive commitment.

Communicating that leaves are to be shed soon allows plants to reduce herbivore load (at a time when nutrient-rich sap is being remobilised from leaves) and allows herbivores to reduce mortality by selecting hosts where leaves will not soon abscise. The authors argued this coevolved signalling system might arise, due to the mutual benefits of such a scenario.

\subsection{Signalling in senescing autumn vs. non-senescing leaves}

The leaf signalling hypothesis was originally focussed upon autumn senescing leaves. More recently, this hypothesis has been considered as a possible explanation for variation in the colouration of newly flushing leaves (Karageorgou and Manetas 2006, Karageorgou et al. 2008) and leaves that maintain foliar anthocyanins throughout the majority of their life (Hughes et al. 2010b, Wong and Srivastava 2011, Cooney et al. 2012). Studying non-senescing leaves allows for an assessment of leaf signalling without the concomitant complex changes in leaf biochemistry and function associated with leaf senescence (Keskitalo et al. 2005, Ougham et al. 2005a). The focus of this thesis will be upon non-senescing leaf systems.

\subsection{Review of criticisms of leaf signalling hypothesis}

Most papers published in support of leaf signalling have presented correlations consistent with either of the first two predictions of the leaf signalling hypothesis (see section 1.6 above). Subsequent reviews have reinforced the claim these findings are evidence in support of leaf signalling (Archetti 2009a, Archetti et al. 2009). However, these papers have received criticism for their lack of consideration of alternative mechanisms that may explain their findings (Wilkinson et al. 2002, Schaefer and Wilkinson 2004, Ougham et al. 2005a, Schaefer and Rolshausen 2006, Schaefer and Gould 2007, Holopainen 2008). Below, I review these alternative mechanisms.

\subsubsection{Prediction 1. Correlation between leaf colour and defensive chemistry}

There are other mechanisms by which leaf colour may correlate with leaf defensive chemistry, aside from leaf signalling:

\subsubsection{Defence indication hypothesis (Schaefer and Rolshausen 2006).}

The biosynthetic pathway that produces anthocyanin pigments also produces the many flavonoid compounds frequently used in plant defence against herbivores (e.g. tannins) (Schaefer and Rolshausen 2006). Additionally, floral anthocyanin 
pigmentation has been demonstrated to be pleiotropically linked with leaf defence (Fineblum and Rausher 1997, Strauss et al. 2004). The 'defence indication' hypothesis posits that foliar anthocyanin pigments primarily function to reduce the impact of abiotic stressors, however biosynthetic and pleiotropic linkages lead to a correlation between anthocyanins and defensive compounds within leaves (Schaefer and Rolshausen 2006), similar to the first prediction of the signalling hypothesis.

Such a correlation would exert selective pressure upon herbivorous insects to perceive and respond to anthocyanin pigments, as a cue of leaf defences. As a result, the defence indication hypothesis subsequently predicts a negative correlation between insects and anthocyanic leaves consistent with prediction 2 of the signalling hypotheis (see below). However, such a correlation would arise through a different proximate mechanism to leaf signalling.

The strength of the pleiotropic linkage between anthocyanins and flavonoid defence may be weaker in plants that do not use flavonoids as their primary defence against herbivores (Fineblum and Rausher 1997). In order to distinguish between the leaf signalling and defence indication hypotheses, one must investigate the mechanism by which pigmentation and defences correlate. This requires the ability to directly quantify defence compounds, ideally in a plant species that does not primarily use flavonoids for plant defence.

\subsubsection{Protection of light sensitive defence compounds (Page and Towers 2002)}

The petioles of Ambrosia chamissonis (Asteraceae) leaves contain high levels of the toxic defence compound thiarubrine A. However, thiarubrine compounds are photolabile, and are degraded after exposure to visible and ultraviolet light. In aboveground $A$. chamissonis tissues, thiarubrine-containing structures are shielded by cells containing anthocyanin pigments (Gould 2004). By comparing thiarubrine A degradation in roots and shoots exposed to light, anthocyanins were demonstrated to contribute to the protection of this plants defensive chemistry (Page and Towers 2002). This study also predicts a positive correlation between leaf colour and defence, although in this case the function of anthocyanins is for light screening rather than for signalling. To exclude the protection of light sensitive defence compounds explanation, studies investigating leaf signalling should focus upon plant species where the primary defence compounds are not photolabile. 


\subsubsection{Prediction 2. Correlation between leaf colour and herbivore abundance and/or feeding damage}

A correlation between leaf colour and reduced insect herbivore abundance or smaller areas of leaf lamina eaten has frequently been interpreted as support for the signalling hypotheses (Hagen et al. 2003, Archetti and Leather 2005, Karageorgou and Manetas 2006, Rolshausen and Schaefer 2007, Ramirez et al. 2008, Wong and Srivastava 2011, Cooney et al. 2012). However, the signalling hypothesis requires that the insects are responding to the leaf colour as a signal. As illustrated above (see section 1.9.1) there are alternative mechanisms by which leaf colour and defences may correlate. Similarly, there are many mechanisms (aside from signalling) by which a correlation between non-green leaf colour and reduced insect abundance or damage can occur; insects may be responding to other properties of anthocyanins, or else responding to other cues that happen to correlate with foliar anthocyanins (Ougham et al. 2005a, Rolshausen and Schaefer 2007, Schaefer and Gould 2007, Holopainen 2008)

\subsubsection{Other properties of anthocyanins}

A correlation between foliar anthocyanins and reduced herbivore damage need not be related to leaf defences. For example, leaf cutter ant (Atta columbica) workers avoided anthocyanic leaf discs from tropical plants (Coley and Aide 1989) - which could be interpreted as support for a correlation between anthocyanins and leaf defences - however, in this system the leaf material is not directly consumed by leafcutter ants but rather is used to propagate fungi for food. The mechanism of deterrence is indirect; the ants avoid eating leaves with fungicidal properties (Coley and Aide 1989).

\subsubsection{Pre-contact correlated cues - VOC}

The leaf signalling hypothesis presumes that visual cues play a primary role in precontact host selection. While it is likely to be an important cue, the role of colour in host selection is poorly studied relative to the role of olfactory cues (Reeves 2011). Plant tissues release volatile organic compounds (VOC), and the sensory appendages of many insects are highly sensitive to these VOC (Dudareva et al. 2006). There has been a vast amount of research into mechanisms by which VOC mediate plant-insect interactions (see section 3.2). 
Colour-correlated VOC may explain patterns in insect damage/abundance through either direct deterrence of herbivores, or indirectly, through tritrophic interactions (Dudareva et al. 2006). VOC released after herbivory are used as cues by some predators and parasitoids of insect herbivores (Kessler and Baldwin 2001, Allmann and Baldwin 2010). It has been repeatedly proposed that leaf colour might correlate with leaf VOC emissions (Archetti and Brown 2004, Ougham et al. 2005b, Holopainen 2008, Döring et al. 2009, Lev-Yadun and Gould 2009). Holopainen et al. (2010) found that green non-senescing leaves emitted different VOC profiles than yellow senescing leaves, and concluded that the green leaves were potentially the better defended against insect herbivores. To my knowledge, no study has investigated the VOC profiles from anthocyanic non-senescing leaves.

\subsubsection{Post-contact correlated cues - Leaf nutrients}

To date, empirical studies of plant quality have focused on the role of plant defences in mediating plant-herbivore interactions and have not considered the influence of variation of host nutritional quality on foraging patterns. There are strong links between leaf colour and nutrient content. In agriculture, anthocyanin accumulation is used as an indicator for certain macronutrient deficiencies, such as phosphorus and nitrogen (Close and Beadle 2003). The timing and intensity of red colouration in autumn senescing maple leaves were correlated with leaf nitrogen content (Schaberg et al. 2003). Therefore, inter-individual differences in leaf redness may be a result of differences in plant nutrition, not necessarily signalling.

In order to grow and reproduce, herbivorous insects must acquire nutrients, and therefore make host selection decisions based upon the nutrient content of their food (Behmer 2009). A diet of poor quality food can lead to impaired development and survival of insect herbivores (Calvo and Molina 2010). Therefore, a correlation between reduced insect abundance, or reduced damage, with leaf colour may be the result of interactions between plant nutritional status and insect nutritional requirements.

\subsection{Gap in knowledge}

As evidenced by the amount of scientific interest it has received, leaf signalling is a compelling and attractive hypothesis. However, at present, we are unable to confirm or refute this hypothesis. This is due to three reasons: 
1) The leaf signalling hypothesis incorporates diverse knowledge areas such as signalling theory, biochemistry, plant-insect interactions, plant and insect physiology, ecology, evolutionary biology (Schaefer and Wilkinson 2004, Archetti et al. 2009). All of these areas have highly developed research fields. The original leaf signalling hypothesis, as well as subsequent models and empirical studies, have not appropriately integrated the knowledge and potential alternate explanations provided by these diverse areas (Wilkinson et al. 2002, Schaefer and Wilkinson 2004, Archetti et al. 2009).

2) Data are scarce and more empirical studies are needed (Archetti et al. 2009). In particular, fitness data pertaining to the $3^{\text {rd }}$ prediction are lacking. No one study has attempted to test all of the leaf signalling predictions in one biological system.

3) The leaf signalling and photoprotective hypotheses for foliar anthocyanins function have been frequently classified as being mutually exclusive (Schaefer and Gould 2007, Archetti et al. 2009). As a result, there have been few attempts to consider the impacts of signalling upon photosynthesis. Only two empirical papers have collected data pertaining to both physiology and leaf signaling (Karageorgou and Manetas 2006, Rolshausen and Schaefer 2007). Due to the inherent light absorbing properties of anthocyanin pigments, these hypotheses are not mutually exclusive (Rolshausen and Schaefer 2007, Schaefer and Gould 2007). Therefore, experiments need to compare the relative influence of foliar anthocyanins on biotic (i.e. deterring herbivores via signaling) vs. abiotic factors.

Given the diverse range of hypotheses for anthocyanin function, especially given the strong support for photoprotective function, and in consideration of the abundant alternative explanations, is it possible to find evidence in support of leaf signalling as an explanation for the maintenance for inter-individual variation in leaf colour?

\subsection{Pseudowintera colorata is an ideal species to study leaf signalling}

A major factor contributing to the imbalance between the numbers of empirical vs. theoretical leaf signalling papers, is the difficulty associated with collecting empirical 
data. The New Zealand native small tree Pseudowintera colorata, possesses several properties which makes it a particularly well suited study system to test if red anthocyanin pigments within leaves function as a visual signal. This species is an ideal plant signaller model because:

1) Its foliage is naturally extremely variable in colour. Its leaves vary from entirely green to entirely red, although most are an irregular mosaic of red and green patches (Fig. 2.1A). Also, in several populations there is marked interindividual variation in leaf colouration.

2) In contrast to the leaf-signalling plant species investigated to date, this species rules out several alternative explanations by virtue of the facts that its primary defence compound (a sesquiterpene dialdehyde called polygodial; Fig. 2.1F) is directly quantifiable, has been repeatedly shown to possess potent antifeedant properties against a variety of phytophagous insects (Asakawa et al. 1988, Gerard et al. 1993, Powell et al. 1993, 1995), is not reported to be photolabile and is biochemically distant from anthocyanins;

3) A large number of the insects associated with $P$. colorata have been documented (http://plant-synz.landcareresearch.co.nz/; accessed August 2010). Cooney et al. (2012) have shown that larvae of the brownheaded leafroller Ctenopseustis obliquana, an important herbivore of $P$. colorata, perceive and respond to red colouration at the leaf margins. Additionally, visual cues were important mediators of feeding decisions for larval Epiphyas postvittana (from the same family as C. obliquana) (Markwick et al. 2012). The larvae of many tortricid moths are important pests of apple and other crops in New Zealand, so research into leaf signalling using this system has the potential to be applied in horticultural/agricultural settings.

\subsection{Aims of the thesis}

The overall aim of this thesis was to carry out a comprehensive test of the hypothesis that foliar anthocyanins function as visual signals, informing insect herbivores of a plant's defensive commitment. Using $P$. colorata, I tested the predictions of the leaf signalling hypothesis (see section 1.6) whilst investigating alternative explanations. 


\subsubsection{Specific Objectives:}

\subsubsection{Test leaf signalling predictions using P. colorata in the field [Chapter 2]}

Using a matched pair design similar to Hagen et al. (2003), I exploited the natural polymorphism in leaf colour of $P$. colorata to test the hypothesis that (i) bright red leaf colour is a reliable signal of a plant's defensive commitment; (ii) insects in the field avoid trees that are brightly coloured; and (iii) the trees with the brightest leaves will have higher fitness.

\subsubsection{Test for olfactory signalling via VOC [Chapter 3]}

Many insects are sensitive to volatile organic compounds (VOCs), however the role of VOCs in plant-herbivore signalling has not been investigated. P. colorata's leaves contain many VOCs (Corbett and Grant 1958). I analysed VOCs released from undamaged, herbivore- and mechanically-damaged red and green leaves of P. colorata, and the olfactory preferences brownheaded leafroller (C. obliquana) larvae. I tested the hypotheses that (i) VOC profiles of $P$. colorata leaves are a reliable indicator of the defensive potential of the leaf, and correlate with anthocyanin content; (ii) leaves with greater red coloration emit a greater amount of sesquiterpenes in their VOC profiles; (iii) any insect deterrent compounds will be released in greater amounts by the red than the green leaves; and (iv) both naïve and experienced $C$. obliquana larvae will prefer the VOCs released from green $P$. colorata leaves.

\subsubsection{Test the influence of foliar anthocyanin pigments on photosynthesis of $P$. colorata [Chapter 4]}

Studies of leaf signalling rarely consider the influence of the light-absorbing properties of non-green pigments upon photosynthesis. I compared the photosynthetic and photoinhibitory responses of red and green leaves from matched, neighbouring pairs of $P$. colorata of contrasting colour. Due to shading caused by anthocyanin pigments, I hypothesised that redder leaves would have a lower maximum photosynthetic assimilation rate and, after stress with white light, show a smaller reduction in photosynthetic efficiency than matched green leaves.

\subsubsection{Test the preferences of insect herbivores in the lab [Chapter 5]}

I used field surveys and laboratory bioassays to identify which invertebrate herbivores are most likely to participate in coevolved leaf-signalling interactions with $P$. colorata. Testing the hypothesis that these herbivores prefer green than red $P$. colorata leaves, I carried out lab trials to assess whether the non-random patterns of feeding damage and larval abundance observed in the field in Chapter 2 resulted from 
feeding and oviposition preferences of herbivores. Also, to test the hypothesis that selecting less-red hosts increases the fitness of participating herbivores, I attempted to quantify the fitness of $C$. obliquana larvae feeding upon red and green $P$. colorata leaves.

\subsubsection{Test for correlations between leaf colour and nutrient content [Chapter 6]} Given that the nutrient status of plants may influence insect feeding preferences, plus the well-established links between leaf colour and plant nutrient status, I investigated the relative levels of leaf $\mathrm{N}$ and $\mathrm{C}$ of red and green $P$. colorata. I tested the hypothesis that red leaves contain lower leaf nitrogen levels. 


\section{Do foliar anthocyanins function as a visual signal in $P$. colorata?}

\subsection{ABSTRACT}

Although anthocyanins are synthesised in the leaves of many plants, the adaptive significance of these pigments is not entirely understood. It has been postulated that their red colours may function as visual signals through coevolution between herbivorous insects and their host tree species, though the hypothesis lacks solid empirical evidence. I exploited the natural polymorphism in leaf colour of Pseudowintera colorata to test three predictions of the leaf signalling hypothesis that (i) bright leaf colour is a signal of a plant's defensive commitment; (ii) insects perceive this signal and thus avoid trees that are brightly coloured; and (iii) the trees with the brightest leaves will have higher fitness. Relative to green leaves, redder foliage contained higher concentrations of polygodial, a sesquiterpene dialdehyde known to have strong antifeedant properties, and incurred less insect feeding damage. Redder trees hosted fewer lepidopteran leafroller larvae than neighbouring matched green trees. Contrary to the predictions of the leaf signalling hypothesis, there was no difference in any of the measured fitness parameters between red and green trees indicating that leaf colour polymorphism in P. colorata is stable. I identified and discussed four nonexclusive scenarios that may explain this polymorphism.

\subsection{INTRODUCTION}

Most leaves are green, but red colouration, predominantly caused by anthocyanin pigments within leaf cell vacuoles, is extremely widespread (Lee 2002). The possible functions of anthocyanin pigments in red leaves have attracted scientific debate over the last decade (Lev-Yadun et al. 2002, Gould 2004, Archetti 2009a, Hughes 2011). Foliar anthocyanins have been posited to mitigate the adverse effects of strong light and low temperatures, to protect against drought stress, and to modulate the redox balance in plants (Steyn et al. 2002, Close and Beadle 2003, Gould 2004, Hatier and Gould 2008b). However, variability in the degree of red leaf colouration within populations, and even between neighbouring individuals of the same species is common (Kytridis et al. 2008, Novak and Short 2010, Hughes 2011, Taulavuori et al. 
2011). Additionally, at a smaller spatial scale, red colouration is often localised to specific areas within leaves or patchily distributed over the leaf lamina (Lee 2007, Wong and Srivastava 2011). Overall, the physiological functions of foliar anthocyanin pigments do not satisfactorily explain the variability in patterns of leaf redness (Archetti and Brown 2004).

As an alternative to the possible physiological functions, Archetti (2000) and Hamilton \& Brown (2001) proposed a novel hypothesis that specifically addressed intra- and inter-species variation in reddening in senescing leaves of deciduous trees. Their hypothesis stated that bright colouration in autumn leaves is a visual signal that deters approaching aphids by honestly revealing a tree's defensive commitment. Individual plants vary in their defensive commitment with the best-defended individuals producing the brightest leaf colouration. Through evolutionary time, herbivores are expected to associate bright red leaf colour with increased defence and evolve a preference for hosts with the greener, less well-defended leaves. Due to differences in host suitability, herbivores that perceive and respond to these cues and select greener plants are expected to display higher fitness than those that do not. Similarly, differences in herbivory between signalling and non-signalling individual trees are expected to result in differences in the fitness of those trees. The leaf signalling hypothesis made three testable predictions: (i) bright leaf colour is a signal of a plant's defensive commitment; (ii) insects perceive this signal and thus avoid trees that are brightly coloured; and (iii) signalling increases the fitness of participating tree and herbivore individuals (Archetti 2000, Hamilton and Brown 2001). Although communication between plants and animals is an active field of study, no paper published to date has examined all the predictions of the leaf signalling hypothesis. This is surprising because communication between plants and herbivores is likely to have far reaching consequences for the study of plant defences.

To qualify as a 'signal' rather than as a 'cue', red leaf colour must be the result of mutually beneficial coevolution between a plant and herbivore. The perception of the red leaf signal must advantage both plant and herbivore. The plant would benefit from a reduced herbivore load, and its insect pests would benefit from information allowing the selection of the least defended host trees (Maynard Smith and Harper 2003, ScottPhillips 2008, Allison and Hare 2009). In contrast, if the perception of red leaf colour 
results in benefits for the insect but not necessarily for the plant, then red leaf colour must be regarded as a cue rather than a signal. A variety of field studies have found correlations between bright non-green leaf colour and reduced numbers of, and/or damage from, insect herbivores (Hagen et al. 2003, Archetti and Leather 2005, Karageorgou and Manetas 2006, Wong and Srivastava 2011, Cooney et al. 2012). However, such correlations are not sufficient evidence to conclude that leaf colour functions as a signal rather than as a cue. To our knowledge, no study has presented fitness data to test this third prediction of the leaf signalling hypothesis. Without data on the relative fitness of differently coloured trees and the relative fitness of individual insect herbivores feeding upon red and green trees, we are unable to confirm leaf signalling. In particular, we are unable to conclude whether deterring herbivores is the adaptive function, rather than merely an effect, of red pigments in leaves (Otte 1974, Allison and Hare 2009).

There are alternative mechanisms, other than signalling, that might explain a link between leaf colouration and reduced insect pressure, and these have rarely been ruled out in previous studies. For example Schaefer and Rolshausen (2006) showed that, in the absence of a known mechanism of insect deterrence, a correlation between red leaves and reduced insect pressure does not provide strong evidence for leaf signalling. Their 'defence indication' hypothesis proposed that red anthocyanin pigments primarily function to reduce the impact of abiotic stressors. However, because they are the end product of the flavonoid biosynthetic pathway that also produces biotic defence compounds (Simpson and Raubenheimer 2001), red pigments may still be a reliable cue of a plant's defensive strength. Alternatively, pigments could be a reliable cue if they function to protect photolabile defence compounds (Page and Towers 2002). Other studies have found correlations between red colouration and the total phenolic content of leaves and interpreted these as evidence in support of the leaf signalling hypothesis (Karageorgou and Manetas 2006, Karageorgou et al. 2008, Hughes et al. 2010b). However, only a small fraction of the total phenolic pool is known to be involved in defence (Lawler et al. 1998, Lawler et al. 1999). It is thought that the primary function of the majority of phenolics is to protect leaves from photodamage (Close and McArthur 2002). With the exception of Cooney et al. (2012), who studied anthocyanin production in the leaf margins of $P$. 
colorata, previous studies of leaf signalling did not identify specific defensive compounds and so were unable to rule out the defence indication hypothesis.

Several reviews have highlighted that anthocyanin pigments are likely to serve multiple functions within leaves (Gould 2004, Lev-Yadun 2006a). Despite this, physiological and signalling hypotheses, although not mutually exclusive (Rolshausen and Schaefer 2007), have often been presented as competing viewpoints, and proponents of each view have criticised the alternative view (Schaefer and Wilkinson 2004, Archetti and Brown 2006, Schaefer and Gould 2007, Schaefer and Rolshausen 2007b, Archetti 2009a, Archetti et al. 2009). It has been repeatedly shown that abiotic stressors can influence anthocyanin pigment production within leaves (Gould et al. 1995, Chalker-Scott 1999, Manetas 2006). With the exception of Rolshausen and Schaefer (2007) and Hagen et al. (2004) studies investigating leaf signalling have neglected to control for environmental effects on colouration.

I used $P$. colorata, a small tree endemic to New Zealand, to test if red anthocyanin pigments within leaves function as a visual signal. This species is an ideal model because $(i)$ the primary defence compound (a sesquiterpene dialdehyde called polygodial; Fig. 2.1F) has been repeatedly shown to possess potent antifeedant properties against a variety of phytophagous insects (Asakawa et al. 1988, Gerard et al. 1993, Powell et al. 1993, 1995), is not reported to be photolabile and is biochemically distant from anthocyanins; (ii) its foliage is naturally extremely variable in colour, leaves vary from entirely green to entirely red, although most are an irregular mosaic of red and green patches (Fig. 2.1A); and (iii) Cooney et al. (2012) have shown that larvae of the brownheaded leafroller Ctenopseustis obliquana, an important herbivore of $P$. colorata, perceive and respond to red colouration at the leaf margins.

I analysed the relationships between leaf colour, anthocyanin and polygodial content and herbivory of leaves collected from two natural populations at distinct locations in New Zealand. Chemistry data and leaf images from the Otago population were collected by Luke Youard and appear in his thesis (Youard 2012). At the Wellington population, where inter-individual differences in leaf redness are particularly strong, I utilised a matched pair design similar to that of Hagen et al. (2004) to test the effect of 
red leaf colouration on insect pressure and plant fitness whilst minimising the influence of environmental variables. I hypothesized that $(i)$ the proportion of red colouration of $P$. colorata leaves is a reliable cue of chemical defences, (ii) redder leaves will suffer reduced insect feeding damage, (iii) red trees will experience less insect pressure than green trees, and $(i v)$ red trees will have higher fitness than green trees. I present these investigations below. Elsewhere, in Appendix B, I document my attempts to quantify the fitness of $C$. obliquana larvae feeding upon red and green $P$. colorata leaves

\subsection{MATERIALS AND METHODS}

\subsubsection{Plant material}

In 2007,15 . colorata plants were randomly selected from a natural population at Waipori Falls Scenic Reserve, Otago, New Zealand (45 54' 13.8" S, $169^{\circ} 59^{\prime} 34.7^{\prime \prime}$ E). From each plant two stems were randomly selected, from which every leaf was removed (Youard 2012). In 2009, further plant material was collected from a natural population of P.colorata at Belmont Trig, Wellington, New Zealand $\left(41^{\circ} 11^{\prime} 0.2^{\prime \prime} \mathrm{S}\right.$, $\left.174^{\circ} 52^{\prime} 25.9^{\prime \prime} \mathrm{E}\right)$. The two populations represent extremes in the range of typical $P$. colorata habitat. P. colorata at Waipori Falls are found in the shaded forest understory (Fig. 2.1C). At Belmont trig P. colorata forms a dense thicket on an exposed hilltop, which received direct sunlight throughout the day (Fig. 1.1D). Fifty plants were randomly selected, stratified over the population (ten plants from each of five $100 \mathrm{~m}$ transects $20 \mathrm{~m}$ apart). From each plant three branches were randomly selected, stratified to represent the lowest, intermediate and highest heights of insertion on the canopy. From each of these stems the oldest, an intermediate, and the youngest fully expanded leaves were collected. A subsample of leaves from each population was used for chemical analyses (Otago: 46 leaves from four randomly selected branches, each from a separate plant; Wellington: 90 leaves from 10 randomly selected plants).

\subsubsection{Leaf colour and insect feeding damage}

Leaf colouration patterns and feeding damage from chewing insects were quantified from digital images of the harvested leaves. The adaxial surface of each was scanned at 300 dpi using a Cannon Canoscan 8400F flatbed scanner, and Adobe Photoshop 5.0 
(San Jose, CA, USA) was used to convert the background of the images from white to black. The area of red lamina was automatically measured by an R-script which converted images from RGB to CIELab colour coordinates, classified and counted pixels according to their colour, and calculated the total leaf area and proportion of leaf area coloured red. This technique followed the method of Ramirez et al. (2008). Mechanical injury and herbivory can induce the biosynthesis of additional anthocyanin pigmentation in leaf tissue immediately adjacent to damage (Gould et al. 2002a). To estimate the colour of the leaf prior to damage, I classified each leaf according to the proportion of red area that was induced redness (i.e. associated with damage). I used this classification to reduce the weighting of induced redness relative to natural leaf colour variation on my calculation of total red leaf area (see Appendix A).

Insect feeding damage was calculated by comparing leaf surface area from each scanned image with an estimate of pre-herbivory leaf surface area created in Image J v1.41 (National Institute of Health, Bethesda, MD, USA). Pre-herbivory surface area was estimated by filling in areas lost to edge herbivory, as well as any holes in the leaf lamina. If leaves had incurred extensive herbivory, their pre-herbivory leaf shape was reconstructed by comparison with the shapes of adjacent leaves on the branch. Leaves that incurred no feeding damage were excluded from statistical analyses that involved leaf herbivory, as it was not known if their lack of damage could be attributed to successfully deterring herbivores or instead to not being encountered by an herbivore.

\subsubsection{Leaf chemistry}

Leaves from the Otago population were individually freeze-dried, weighed, and ground to a powder. Half of each sample was extracted in $3 \mathrm{M} \mathrm{HCL}: \mathrm{H}_{2} \mathrm{O}: \mathrm{MeOH}$ $(1: 3: 16, \mathrm{v} / \mathrm{v} / \mathrm{v})$, centrifuged, and absorbances at $522 \mathrm{~nm}\left(A_{\max }\right)$ and $653 \mathrm{~nm}$ of the supernatant measured using a Pharmacia Biotech Ultraspec 2000 UV/Visible spectrophotometer (Pharmacia Biotech, Uppsala, Sweden). Anthocyanin concentrations were estimated as $A_{522}-0.24 A_{653}$ per unit dry weight. The remaining half of each sample was used for polygodial quantification as described below (Youard 2012). 
For leaves from the Wellington population both polygodial and anthocyanin concentrations were measured by HPLC using a procedure modified after Wayman et al. (2010). These analyses were carried out by John van Klink at Otago University. Leaf samples were freeze dried for $24 \mathrm{~h}$ and ground to a powder. Subsamples ( $\sim 10$ $\mathrm{mg})$ were extracted with rectified spirits $(1 \mathrm{ml})$ containing $\mathrm{C}_{10}$ anilide $(200 \mu \mathrm{g})$ as an internal standard (Perry et al. 1996), briefly sonicated ( 30 s), stirred overnight, and then filtered through a $0.45 \mu \mathrm{m}$ PTFE filter. Analyses were performed on an Agilent 1100 HPLC (Agilent Technologies, Santa Clara, CA) fitted with a diode array detector, using a Luna (II) $250 \times 3$ mm RP-18 (5 $\mu \mathrm{m}$ ) column (Phenomenex, Torrance, CA) with a SecurityGuard ${ }^{\mathrm{TM}} 4 \times 2 \mathrm{~mm}$ C18 guard column (Phenomenex, Torrance, CA) at $30^{\circ} \mathrm{C}$. Peaks were monitored at 206, 230, 280, 330 and $530 \mathrm{~nm}$. The mobile phase was $\mathrm{MeCN}$ in $\mathrm{H}_{2} \mathrm{O}$, both containing formic acid (0.1\%): $5 \% \mathrm{MeCN}$ at 0 $\mathrm{min}, 100 \%$ at $30 \mathrm{~min}, 5 \%$ at $35 \mathrm{~min}, 5 \%$ at $40 \mathrm{~min}$. The flow rate was $0.5 \mathrm{ml} / \mathrm{min}$ with injection volumes of $5 \mu 1$. Quantification of an isolated reference sample of polygodial against the internal standard was performed at $230 \mathrm{~nm}$. Quantification of anthocyanins was achieved by calibrating with a commercial source of cyanidin-3-Oglucoside (Extrasynthese, Genay, France) at $530 \mathrm{~nm}$ versus the internal standard at $206 \mathrm{~nm}$. Total anthocyanins were reported as cyanidin-3-O-glucoside equivalents. Other compounds were identified by their eluting times, including another sesquiterpene dialdehyde, 9-deoxymuzigadial, and the polyphenol, chlorogenic acid (CGA). Levels of each compound identified were reported as $\mathrm{mg}$ (or $\mu \mathrm{g}$ for anthocyanins)/g dry weight of material extracted.

\subsubsection{Pair-wise comparison of herbivore pressure}

I selected 30 pairs of $P$. colorata trees at the Wellington site, following the methods of Hagen et al. (2004). This design allowed me to investigate the effect of leaf colour on herbivory whilst minimising the influence of abiotic factors. Each pair contained one tree whose leaves were predominantly green and one tree whose leaves were much redder (Fig. 2.1B). The individuals in each pair were located no more than $2 \mathrm{~m}$ apart from one another, and closely matched in size, aspect, exposure to direct sunlight and prevailing winds. Canopy surface area of each plant was measured using a $0.0672 \mathrm{~m}^{2}$ quadrat. The quadrat was placed upon the canopy and rotated edge to 
edge in parallel rows over the entire exterior surface of the plant. Subsequent measures of herbivory pressure and plant fitness are reported per $\mathrm{m}^{2}$ canopy surface area. Very large $P$. colorata trees $\left(>\sim 6 \mathrm{~m}^{2}\right)$ were excluded.

Because a previous season's herbivory might influence herbivory pressure and plant fitness (Marquis 1984), a survey of leaf herbivory was conducted at the beginning of this study. From each individual in the matched-pair experiment, 100 fully expanded leaves were haphazardly selected. Each leaf was scored as having incurred chewing insect feeding damage or not. The proportionate number of leaves that had incurred herbivory was recorded.

New Zealand native leafroller caterpillars from the family Tortricidae were the most abundant of the known chewing insect herbivores of $P$. colorata at the Wellington field site (http://plant-synz.landcareresearch.co.nz/; accessed August 2010). From late Spring to early Summer (November to December) 2010, I counted the caterpillars visible on every leaf on all 60 trees, and recorded evidence of leafroller caterpillar presence (leaves rolled or stuck together with silk, Fig. 2.1E). Caterpillars were left undisturbed so that I could observe the impact of their feeding on plant fitness at the end of the season.

\subsubsection{Estimation of plant fitness}

Several features of plant reproduction make quantifying individual's life-time fitness extremely difficult, especially in natural conditions (Primack and Kang 1989). In order to estimate fitness, researchers commonly measure components of fitness such as: leaf area, flower set, pollination success, fruit set, seed set, seed size, seed viability, seedling survival, seedling fecundity (Primack and Kang 1989, Herrera 1991, Stowe et al. 2000). I collected data regarding components of the reproductive effort of matched pairs of $P$. colorata, with the assumption that individuals that produced the greater number or mass of seeds, would have the greater probability of leaving more offspring.

From the same 30 pairs, during late summer to early autumn (February/March 2011, before the majority of fruits were fully ripened) each stem was inspected and the total 
number of fruits counted. $P$. colorata produces small (3-5 $\mathrm{mm}$ in diameter) fruits that change colour from green to very dark red or black when ripe. Fruit numbers were divided by each tree's canopy area to compensate for small differences in stature. Close to 30000 fruits from 30 pairs were counted. For 26 pairs, 20 randomly selected fruits from each plant were dissected and their seeds counted. These seeds were then cleaned, dried, and their mean dry weights determined to test for differences in the number of seeds per fruit and seed weight. The number of seeds per $\mathrm{m}^{2}$ canopy area of each tree was estimated by multiplying mean numbers of seeds per fruit by total number of fruits per $\mathrm{m}^{2}$ canopy area. Seed mass per $\mathrm{m}^{2}$ canopy area of each tree was estimated by multiplying mean seed weight by estimated total number of seeds per $\mathrm{m}^{2}$ canopy area.

\subsubsection{Statistical analyses}

The different ways leaves were sampled from the Otago and Wellington populations, combined with the fact that chemical analyses were carried out on a subsample of leaves, precluded a multivariate analysis to compare the relationships between leaf colour, chemistry and herbivory at the two populations. Instead, I described patterns of variation in leaf colour, chemistry and herbivory between and within $P$. colorata individuals from Wellington and Otago. I then used multiple linear mixed effect models to analyse the relationships between leaf colour, chemistry and herbivory within each population.

\subsubsection{Description of inter- and intra-individual patterns}

Individual means and coefficient of variation $(\mathrm{CoV}=($ standard deviation $/$ mean $) * 100)$ were plotted to describe the patterns of variability of leaf traits and herbivory between and within populations. $\mathrm{CoV}$ is useful for making comparisons of the within-plant variability of traits among individuals (Herrera 2009). In Wellington, leaf trait variation within-individuals was investigated along two axes: variation across three different heights within each tree (corresponding to exposure to environmental variables such as light, wind and cooler temperatures) and variation with leaf age along a stem at each height (corresponding to leaf ontogeny). Differences in traits between heights and between leaf ages at each height were analysed using a nested ANOVA design (leaf age nested within height) in PSAW/SPSS Version 18.0 (Chicago, IL, USA). Equality of variance of each variable was assessed using 
Levene's test, natural log transformations were applied when necessary. Where there were differences within branches, separate ANOVA analyses with Tukey's HSD (honestly significant difference) test were applied post-hoc.

Because the $P$. colorata trees from Otago were beneath the forest canopy and were approximately equally shaded, branch height did not represent differences in exposure as in Wellington. Instead, to get a finer resolution of leaf trait variation with ontogeny, leaf age was recorded as node number along a stem (an ordinal variable) rather than classified categorically as in Wellington. Nodes were counted starting from the youngest fully expanded leaf.

\subsubsection{Relationships between colouration, chemistry and herbivory of P. colorata} leaves from Wellington and Otago

General linear mixed-models were used to assess the relationships between polygodial and leaf pigment, polygodial and leaf redness, and between the proportion of leaf area removed by chewing insects (herbivory) and leaf redness, using the 'Ime' command of the nlme package in $\mathrm{R}$, with maximum likelihood estimation (R Core Team 2013). To test if anthocyanin had direct effects on palatability and, therefore, leaf damage, I carried out a further analysis including both anthocyanin and polygodial concentrations as main effects in a mixed-effects model predicting herbivory. Following a Shapiro-Wilk test, the response variables for each model were transformed to improve normality (polygodial: ln; herbivory: arcsine square root). The models included nested random factors (Wellington: individual nested within transect; Otago: branch nested within individual) to reflect the different sampling methodologies at the two sites and to account for the lack of independence of leaves collected from the same plant. The Fligner-Killen test in R was used to compare variability of herbivory for different amounts of leaf redness.

\subsubsection{Differences in herbivore pressure and reproductive effort within matched} pairs of $\mathrm{P}$. colorata at Wellington

Differences in herbivore pressure and reproductive effort between pairs of trees were first examined for normality using a Shapiro-Wilk test, and then analysed using either a paired t-test or Wilcoxon signed ranks test in PSAW/SPSS Version 18.0 (Chicago, IL, USA). 


\subsection{RESULTS}

At both sites the proportion of red colouration varied considerably among leaves. Red leaf area ranged from a barely visible $0.03 \%$ to almost entirely red, with both populations having leaves with greater than $96 \%$ of the adaxial surface area coloured red. However for most leaves, red colouration was concentrated in leaf margins and small blotches over the lamina (red leaf area: Wellington median $=1.8 \%$, Interquartile range $\left[\mathrm{IQR}=\mathrm{Q}_{1}\right.$ to $\left.\mathrm{Q}_{3}\right]=0.6-4.8 \%$; Otago median $=1.0 \%, \mathrm{IQR}=0.5-2.4 \% ;$ Fig. 2.1A). 


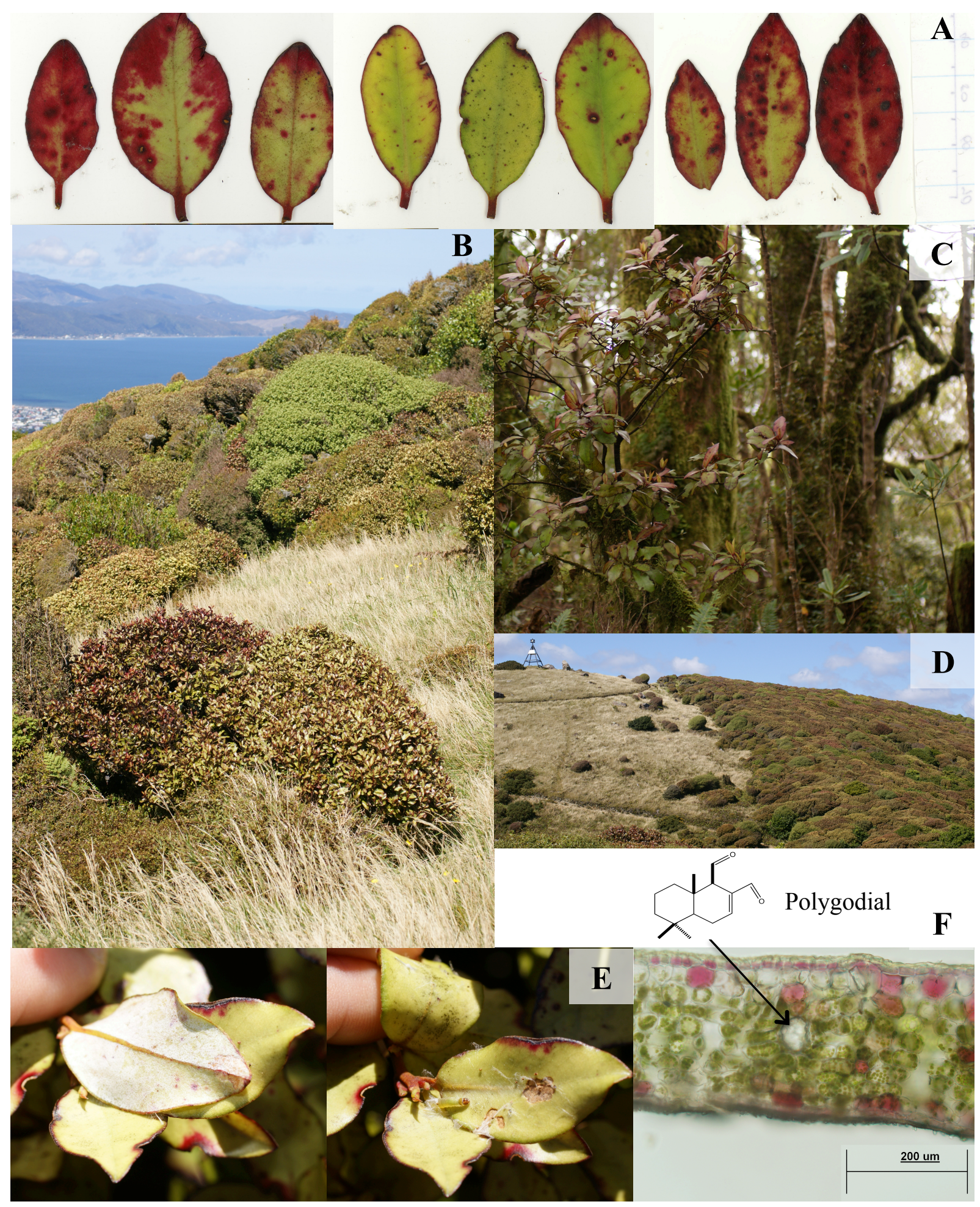

Fig. 2.1 (A) Patterns of colouration of $P$. colorata leaves; (B) a matched pair of $P$. colorata trees contrasting in colour; photographs representative of (C) Otago and (D) Wellington sites; (E) Ctenopseustis sp. leafroller caterpillar within P. colorata leaves; and $(\mathbf{F})$ transverse section through a $P$. colorata leaf showing idioblast, red anthocyanin pigments and structure of polygodial. 


\subsubsection{Comparison of leaf colour, chemistry and herbivory of P. colorata individuals from Wellington and Otago}

\subsubsection{Colour}

Wellington and Otago populations of $P$. colorata did not differ in either average redness of individuals (Mann-Whitney $\mathrm{U}, P=0.493$ ) or variability in leaf redness within individuals (Mann-Whitney $\mathrm{U}, P=0.069$, Fig. 2.2A). Anthocyanin concentrations within the leaves of plants from Wellington and Otago were quantified by different methods and therefore are not easily comparable. However, the range of anthocyanin concentrations of leaves within individuals was significantly higher in Wellington than Otago (Mann-Whitney U, $P=0.007$, Fig. 2.2B).

\subsubsection{Chemistry}

Consistent with Wayman et al. (2010), P. colorata leaves from the Wellington and Otago populations contained both polygodial and 9-deoxymuzigadial. Wellington $P$. colorata trees had 2.5 times higher average polygodial concentrations within their leaves (Mann-Whitney $\mathrm{U}, P=0.011$ ), but less within-tree variability in polygodial than those in Otago (Mann-Whitney $\mathrm{U}, P=0.024$, Fig. 2.3A). Similarly, concentrations of 9-deoxymuzigadial were almost 2.5 times higher in Wellington $P$. colorata trees than in those from Otago (Mann-Whitney $\mathrm{U}, P=0.005$ ), however there was no difference in within-individual variation between these populations (MannWhitney $\mathrm{U}, P=0.888$, Fig. $2.3 \mathrm{~B})$. The range of within-individual variation in leaf chlorogenic acid concentration was similar to the range in variation in polygodial and 9-deoxymuzigadial in Wellington (Fig. 2.3C).

\subsubsection{Herbivory}

Otago P. colorata trees sustained over four times higher average herbivory levels than those from Wellington (Mann-Whitney $\mathrm{U}, P<0.001$ ), but there was no difference in within-individual variation in herbivory between these populations (Mann-Whitney $\mathrm{U}, P=0.071$, Fig. 2.3C). 

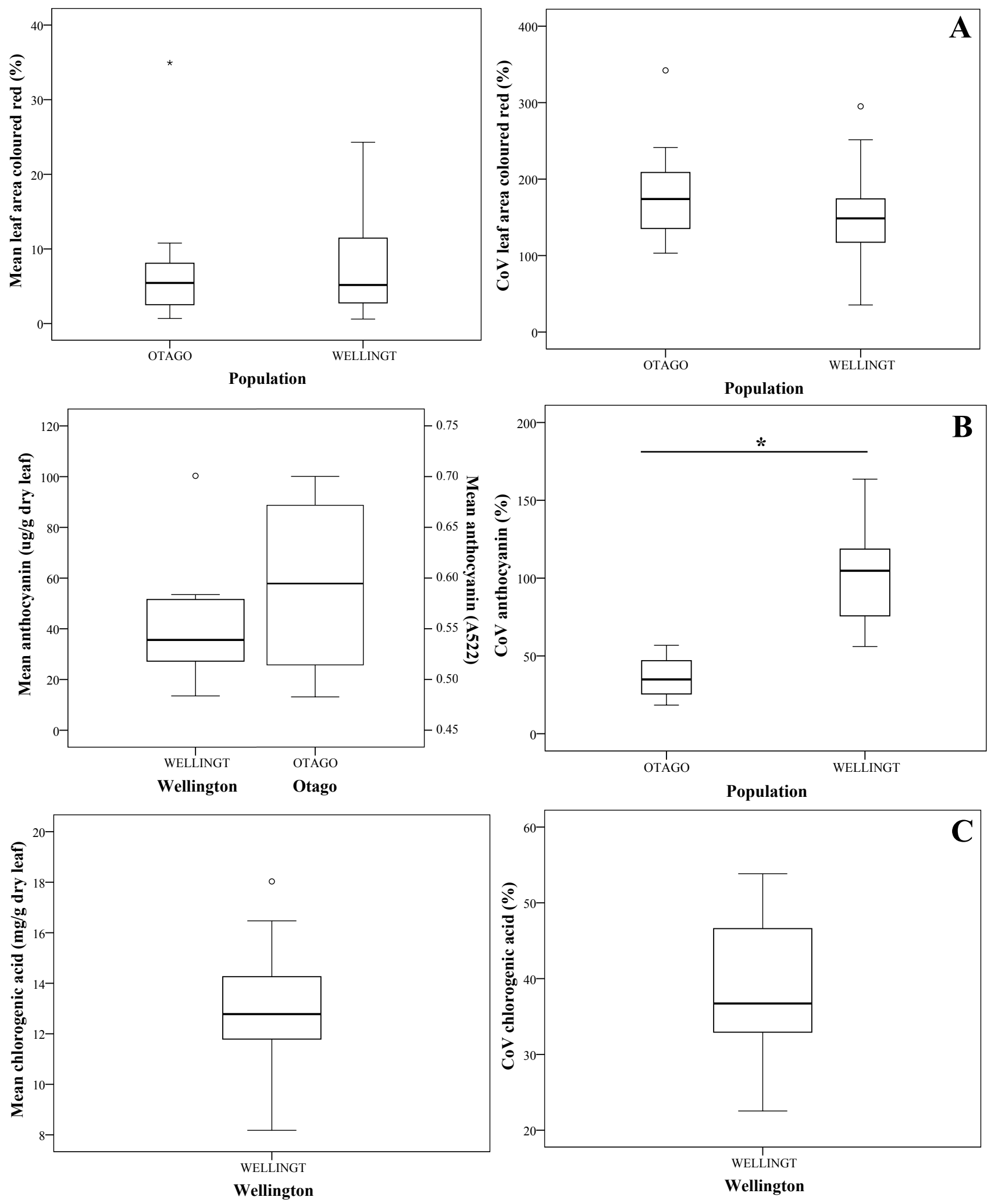

Fig. 2.2 Box-and-whisker plots of individuals' mean (left) and coefficient of variation (right) for $(\mathbf{A})$ leaf redness $\left(\mathrm{n}_{\text {Wellington }}=50, \mathrm{n}_{\text {Otago }}=15\right),(\mathbf{B})$ anthocyanin $\left(\mathrm{n}_{\text {Wellington }}=10, \mathrm{n}_{\text {Otago }}=4\right)$ and $(\mathbf{C})$ chlorogenic acid $\left(\mathrm{n}_{\text {Wellington }}=10\right)$ concentrations (DW) of P. colorata trees at Otago and Wellington. Asterisks indicate statistical significance (Mann-Whitney $\mathrm{U} ; P<0.05)$. 

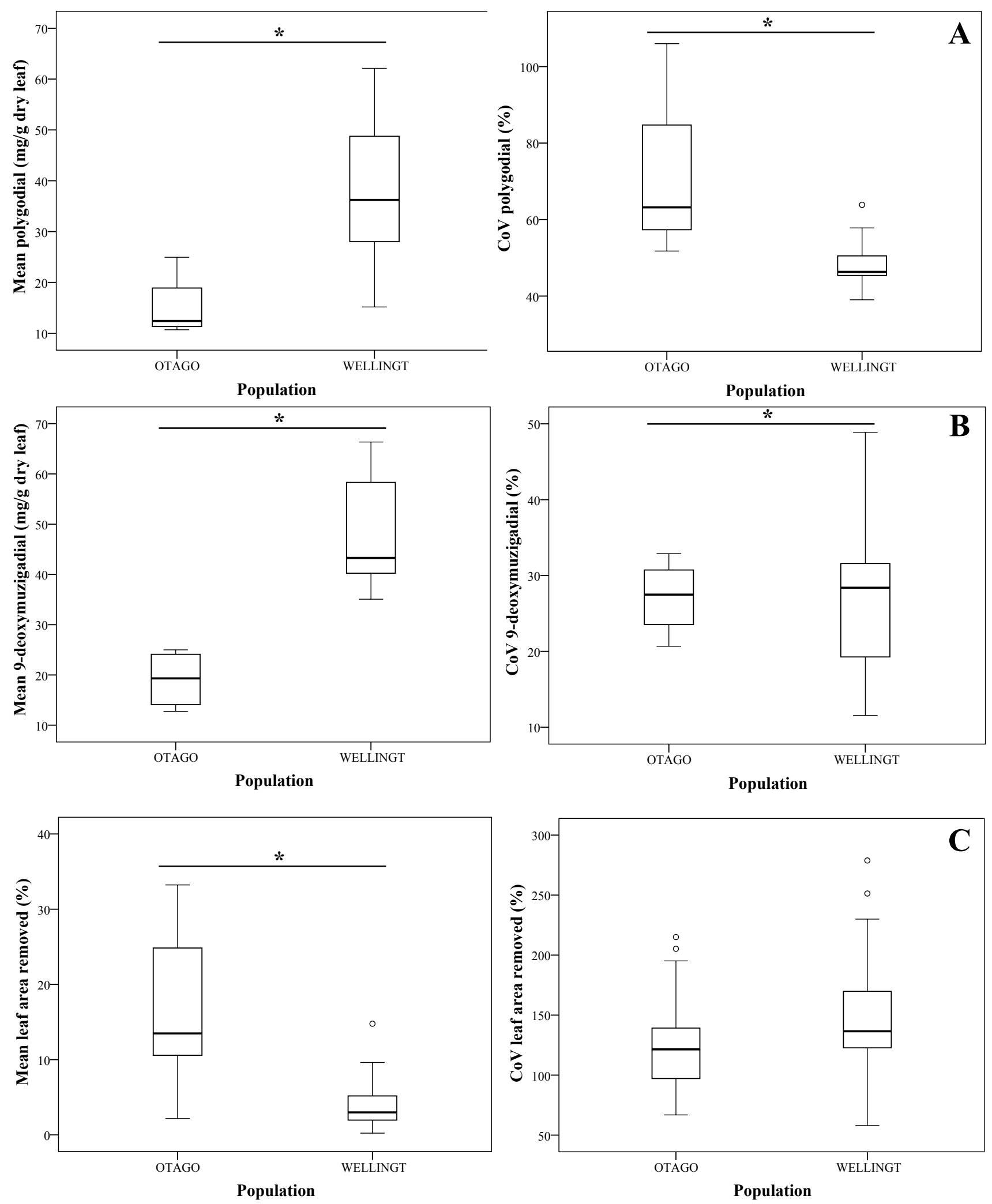

Fig. 2.3 Box-and-whisker plots of individuals' mean (left) and coefficient of variation (right) for (A) polygodial and (B) 9-deoxymuzigadial concentrations (DW, $\left.\mathrm{n}_{\text {Wellington }}=10, \mathrm{n}_{\text {Otago }}=4\right)$ and $(\mathbf{C})$ herbivory levels $\left(\mathrm{n}_{\text {Wellington }}=50, \mathrm{n}_{\text {Otago }}=15\right)$ from $P$. colorata trees at Otago and Wellington. Asterisks indicate statistical significance (Mann-Whitney U; $P<0.05$ ). 
2.4.2 Within-individual patterns in leaf colour, chemistry and herbivory of $P$. colorata trees from Wellington

Leaves from branches from higher in the canopy tended to be redder $\left(\mathrm{F}_{2,440}=129.0, P\right.$ $<0.001$, Fig. 2.4A) and contained higher dry weight concentrations of anthocyanin $\left(\mathrm{F}_{2,81}=54.349, P<0.001\right.$, Fig. $\left.2.4 \mathrm{~B}\right)$ and chlorogenic acid $\left(\mathrm{F}_{2,80}=11.537, P<0.001\right.$, Fig. 2.4C). 9-deoxymuzigadial concentrations showed the opposite trend and decreased with height $\left(\mathrm{F}_{2,81}=3.336, P=0.041\right.$, Fig. 2.5B $)$. Concentrations of polygodial $\left(\mathrm{F}_{2,81}=2.565, P=0.083\right.$, Fig. $\left.2.5 \mathrm{~A}\right)$ and amounts of herbivory $\left(\mathrm{F}_{2,371}=\right.$ $0.607, P=0.55$, Fig. $2.5 \mathrm{C}$ ) of leaves did not vary consistently among branches at different heights.

For any one branch, younger leaves tended to be redder $\left(\mathrm{F}_{6,440}=12.6, P<0.001\right.$, Fig. 2.4A) with higher concentrations of anthocyanin $\left(\mathrm{F}_{6,81}=2.427, P=0.033\right)$ and polygodial $\left(\mathrm{F}_{6,81}=2.482, P=0.024\right.$, Fig. $\left.2.5 \mathrm{~A}\right)$ than older leaves. There was no statistical difference in the concentrations of chlorogenic acid $\left(\mathrm{F}_{6,80}=0.175, P=\right.$ 0.983, Fig. 2.4C), 9-deoxymuzigadial $\left(\mathrm{F}_{6,81}=0.879, P=0.514\right.$, Fig. 2.5B) or amount of herbivory $\left(\mathrm{F}_{6,371}=0.901, P=0.49\right.$, Fig. 2.5C) across leaves of different ages.

\subsubsection{Within-individual patterns in leaf colour, chemistry and herbivory of $P$. colorata trees from Otago}

In Otago, leaf redness (exponential regression, $\mathrm{R}^{2}=0.0351, P<0.001, \mathrm{n}=486$, Fig. 2.6A), anthocyanin (logarithmic regression, $R^{2}=0.242, P<0.001, \mathrm{n}=46$, Fig. 2.6B) and polygodial concentrations (logarithmic regression, $R^{2}=0.327, P<0.001, \mathrm{n}=46$, Fig. 2.7A) decreased with leaf age. 9-Deoxymuzigadial concentrations did not change with leaf age (logarithmic regression, $R^{2}=0.006, P=0.618, \mathrm{n}=46$, Fig. 2.7B). No data was collected for chlorogenic acid. These results are consistent with those for Wellington populations, with the exception of herbivory, which showed a weak but significant increase with leaf age in Otago (exponential regression, $\mathrm{R}^{2}=$ 0.052, $P<0.001, \mathrm{n}=408$, Fig. 2.7C). 

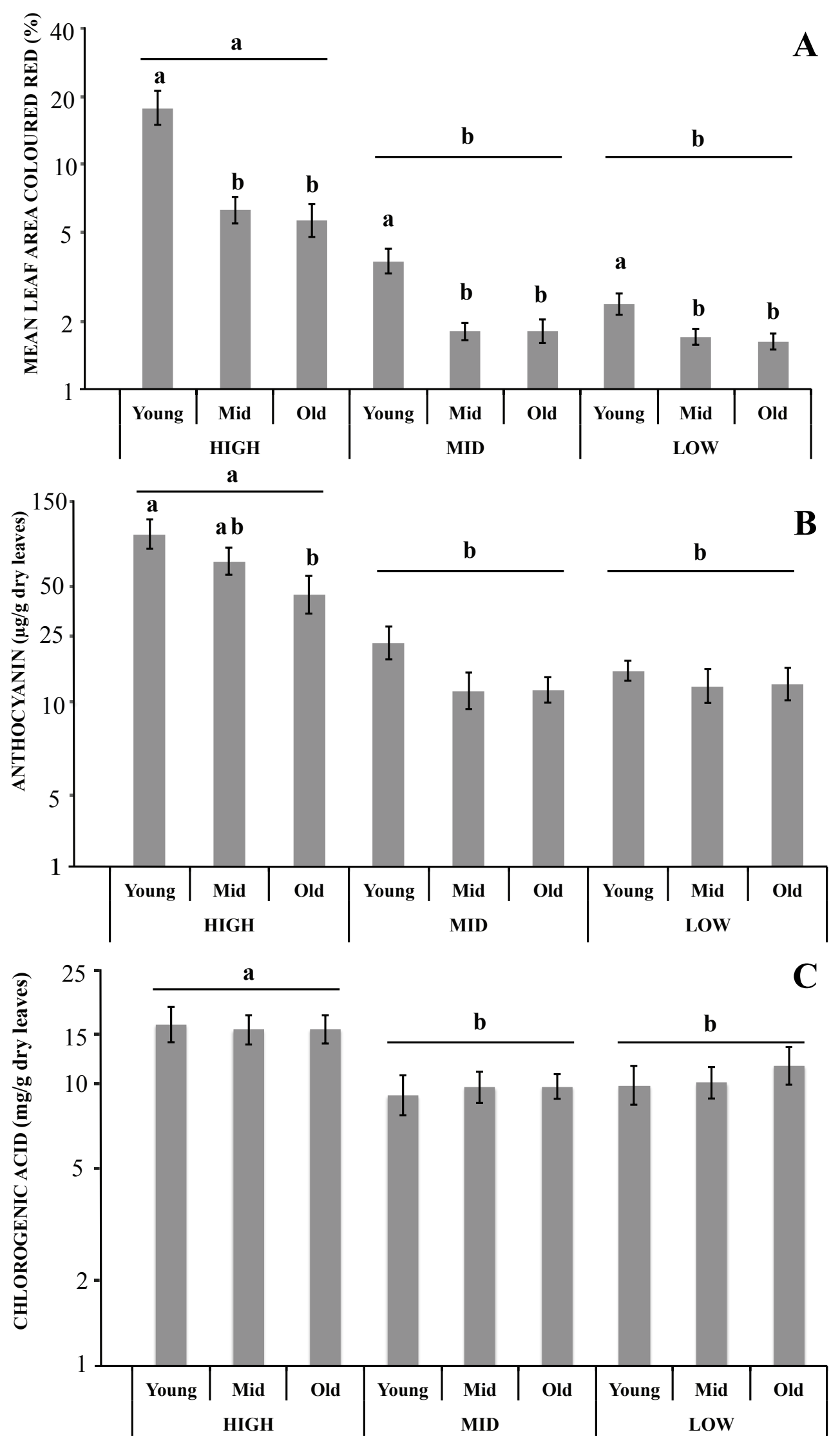

Fig. 2.4 Within-individual patterns in natural log transformed (A) leaf colour $(\mathrm{n}=$ 449), and (B) anthocyanin and (C) chlorogenic acid concentrations (DW, $n=90)$ of young, intermediate and old fully expanded leaves from three branches at different heights of insertion of $P$. colorata trees from Wellington (Y-axis labels backtransformed). Means \pm SE. Different letters indicate statistical significance (nested ANOVA, $P<0.05)$. 

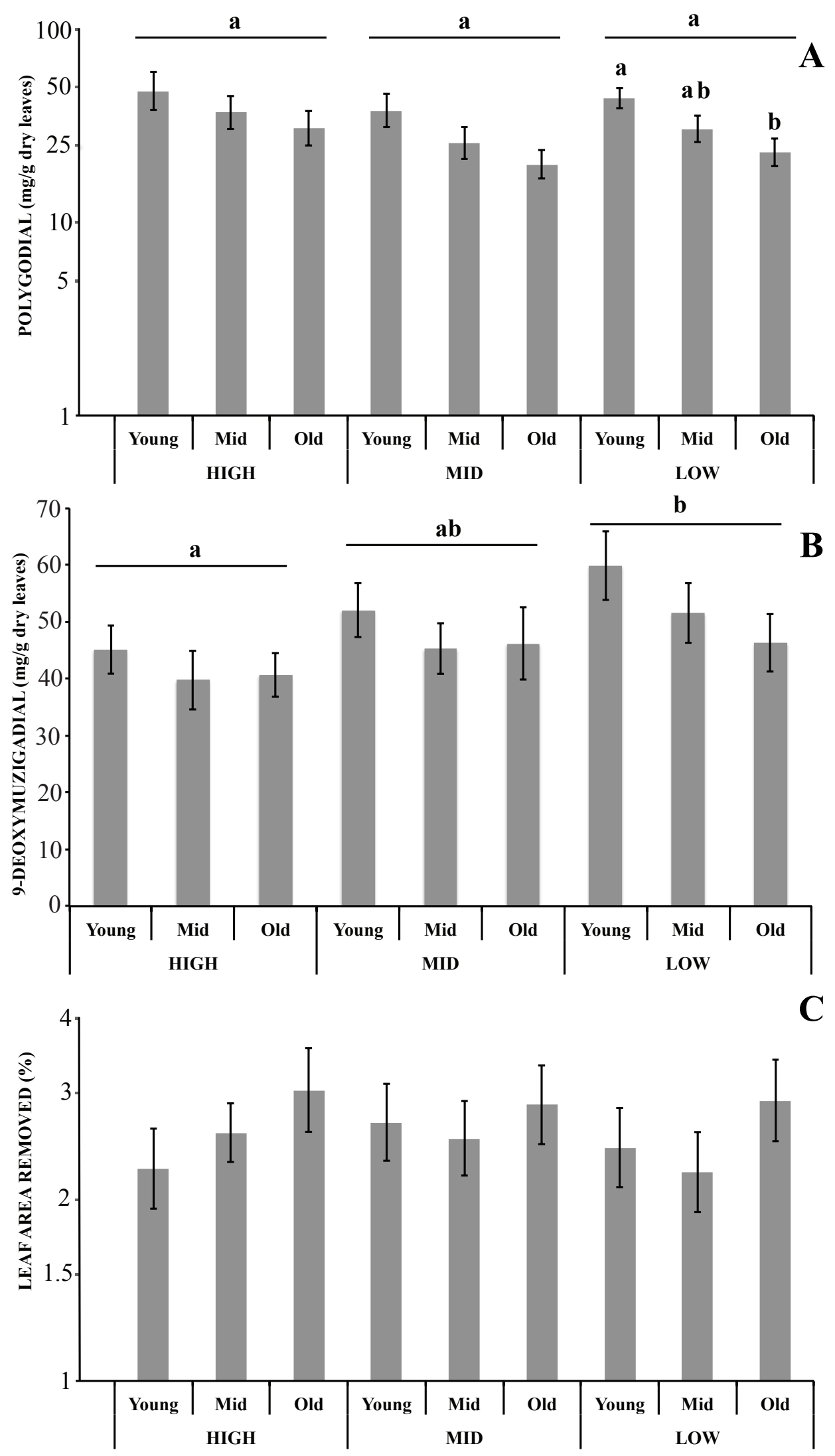

Fig. 2.5 Within-individual patterns in natural log transformed (A) polygodial and (B) 9-deoxymuzigadial concentrations (DW, $n=90)$ and $(\mathbf{C})$ herbivory levels $(n=380)$ of young, intermediate and old fully expanded leaves from three branches at different heights on $P$. colorata trees from Wellington (Y-axis labels back-transformed). Means \pm SE. Different letters indicate statistical differences (nested ANOVA, $P<$ $0.05)$. 

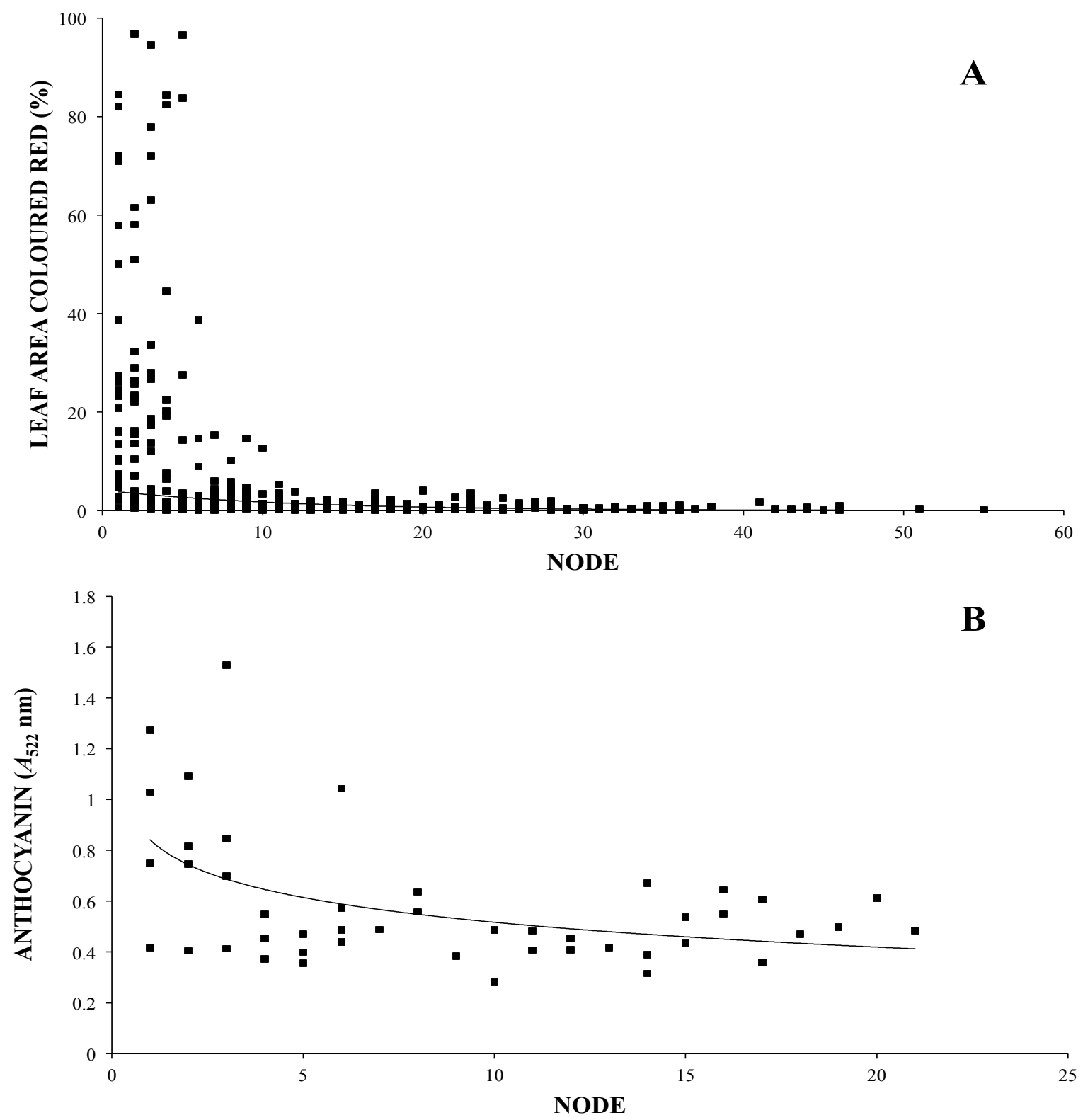

Fig. 2.6 Within-individual patterns in $(\mathbf{A})$ leaf colour $(\mathrm{n}=486)$ and $(\mathbf{B})$ anthocyanin concentration $(\mathrm{n}=46)$ of fully expanded $P$. colorata leaves of different ages from Otago. 

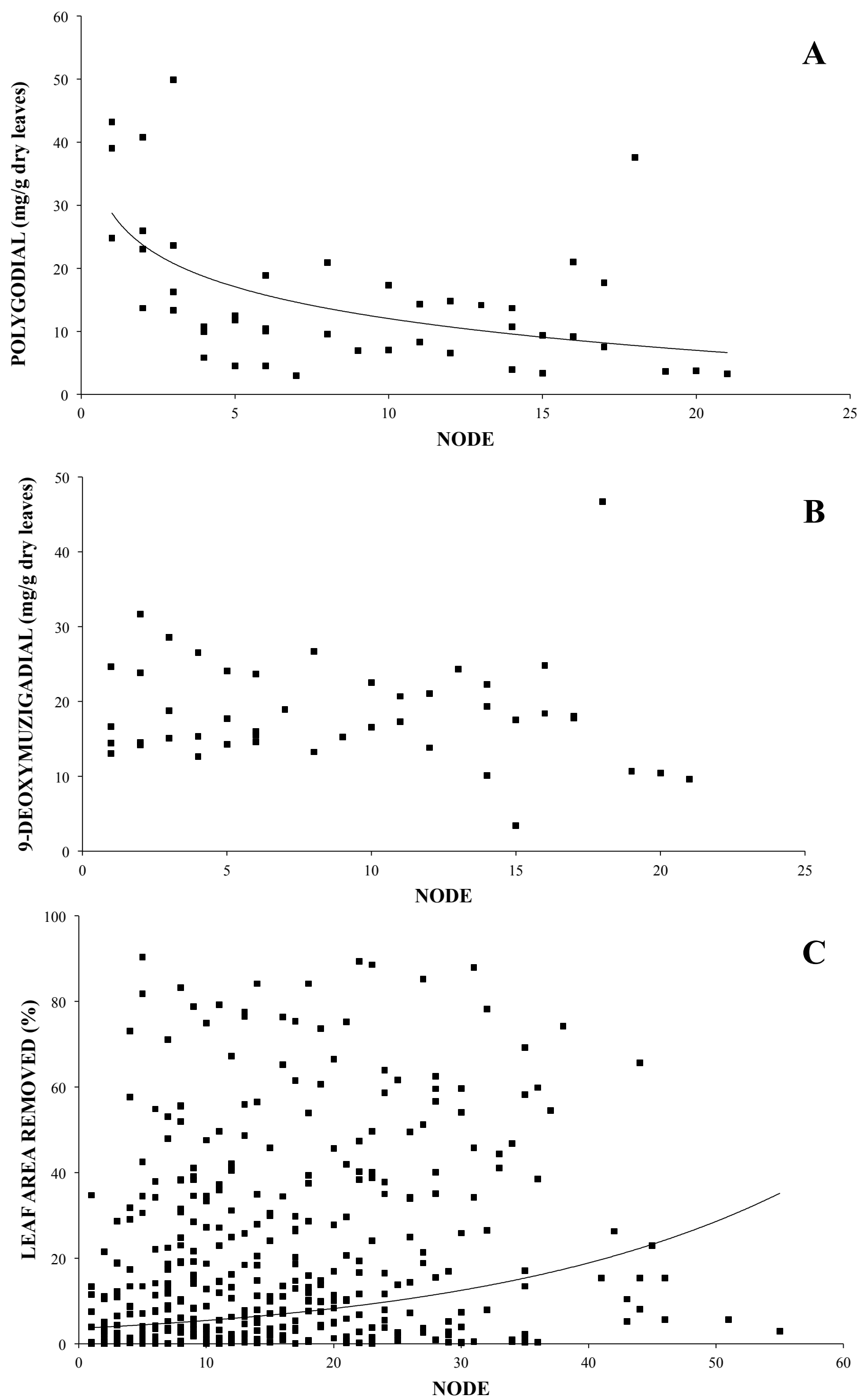

Fig. 2.7 Within-individual patterns in (A) polygodial and (B) 9-deoxymuzigadial concentrations $(\mathrm{n}=46)$ and $(\mathbf{C})$ herbivory levels $(\mathrm{n}=408)$ of fully expanded $P$. colorata leaves of different ages from Otago. 
2.4.4 Relationships between leaf redness, chemistry and herbivory from P. colorata trees in Wellington and Otago

2.4.4.1 Red colouration in P. colorata leaves is a reliable cue of chemical defences At both sites, leaves with the higher concentration of anthocyanin pigments generally held the higher levels of polygodial (Fig. 2.8 \& Table 2.1). Differences in anthocyanin concentration between leaves corresponded to differences in the outward appearance of those leaves. Leaves with high anthocyanin concentrations had a higher proportion of leaf area coloured red (Fig. $2.8 \&$ Table 2.1). After controlling for leaf redness that had resulted from an induced response to leaf damage, the proportion of red coloured leaf area reliably indicated polygodial concentrations of $P$. colorata leaves (Fig. 2.8 \& Table 2.1). 
Table 2.1 Results from general linear mixed-model analyses of the relationships between the leaf redness, chemistry and herbivory from $P$. colorata trees in Wellington and Otago. The response variables for each model were transformed to improve normality (polygodial: ln; herbivory: arcsine square root). Models included random factors (Wellington: individual nested within transect; Otago: branch nested within individual) but those effects are not presented below. Asterisk indicates statistical significance $(P<0.05)$.

\begin{tabular}{|c|c|c|c|c|c|}
\hline \multicolumn{2}{|l|}{ Model } & d.f.NUM & d.f.DENOM & $F$-value & $P$ \\
\hline \multicolumn{6}{|c|}{ (a) Polygodial $\sim$ anthocyanin } \\
\hline \multicolumn{2}{|l|}{ Wellington } & 1 & 79 & 17.9 & $0.0001 *$ \\
\hline \multicolumn{2}{|l|}{ Otago } & 1 & 41 & 17.3 & $0.0002 *$ \\
\hline \multicolumn{6}{|c|}{ (b) Polygodial $\sim$ redness } \\
\hline \multicolumn{2}{|l|}{ Wellington } & 1 & 79 & 14.0 & $0.0003 *$ \\
\hline \multicolumn{2}{|l|}{ Otago } & 1 & 41 & 25.8 & $<0.0001 *$ \\
\hline \multicolumn{6}{|c|}{ (c) Herbivory $\sim$ redness } \\
\hline \multicolumn{2}{|l|}{ Wellington } & 1 & 329 & 5.5 & $0.0195 *$ \\
\hline \multicolumn{2}{|l|}{ Otago } & 1 & 378 & 8.3 & $0.0042 *$ \\
\hline \multicolumn{6}{|c|}{ (d) Herbivory $\sim$ anthocyanin + polygodial + anthocyanin* polygodial } \\
\hline \multirow[t]{3}{*}{ Wellington } & Anthocyanin & 1 & 65 & 0.004 & 0.9521 \\
\hline & Polygodial & 1 & 65 & 4.3 & $0.0400 *$ \\
\hline & Anthocyanin:polygodial & 1 & 65 & 0.3 & 0.5642 \\
\hline \multirow[t]{3}{*}{ Otago } & Anthocyanin & 1 & 28 & 0.007 & 0.9350 \\
\hline & Polygodial & 1 & 28 & 0.03 & 0.8671 \\
\hline & Anthocyanin:polygodial & 1 & 28 & 3.0 & 0.0928 \\
\hline
\end{tabular}



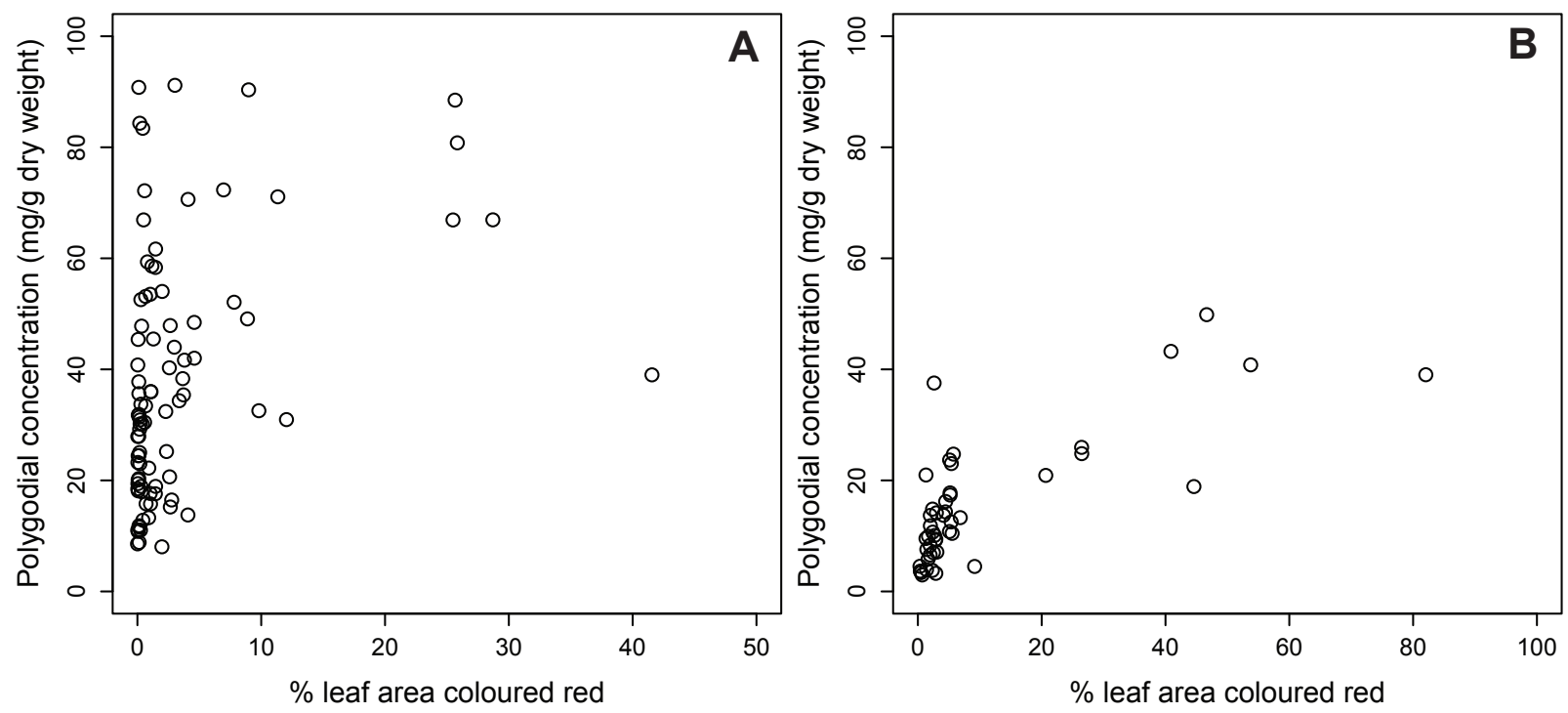

Fig. 2.8 Proportionate leaf redness and polygodial concentrations in leaves of $P$. colorata from (A) Wellington $(n=90)$ and $(B)$ Otago $(n=46)$. Untransformed data presented here, but analyses were conducted on ln-transformed polygodial data.

\subsubsection{Redder P. colorata leaves incur less insect feeding damage}

After controlling for the proportion of leaf redness that resulted from an induced response to leaf damage, the redder $P$. colorata leaves incurred less feeding damage from chewing insects than did green leaves at both locations (Fig. $2.9 \&$ Table 2.1). Many leaves had no evidence of herbivory (Wellington, 116 of 748 leaves; Otago, 78 of 486 leaves). Of the leaves with damage, the amount of leaf area consumed was much lower at the Wellington site (mean feeding damage $=3.4 \% \pm 0.25 \%, \max =$ $56.5 \%)$ than at the Otago site $(19.6 \% \pm 1.12 \%, \max =90.4 \%)$.

In addition to incurring lower amounts of feeding damage, redder leaves from both populations experienced much less variability in feeding damage. There was a critical proportionate red leaf area above which variance in leaf damage was dramatically reduced; this was $4.25 \%$ for leaves from Wellington (Fligner-Killeen, d.f. $=1, P<$ 0.001 ) and $4 \%$ for those from Otago (d.f. $=1, P=0.013$ ). Furthermore, in both populations, no leaf with greater than $30 \%$ red area incurred more than $15 \%$ damage. 

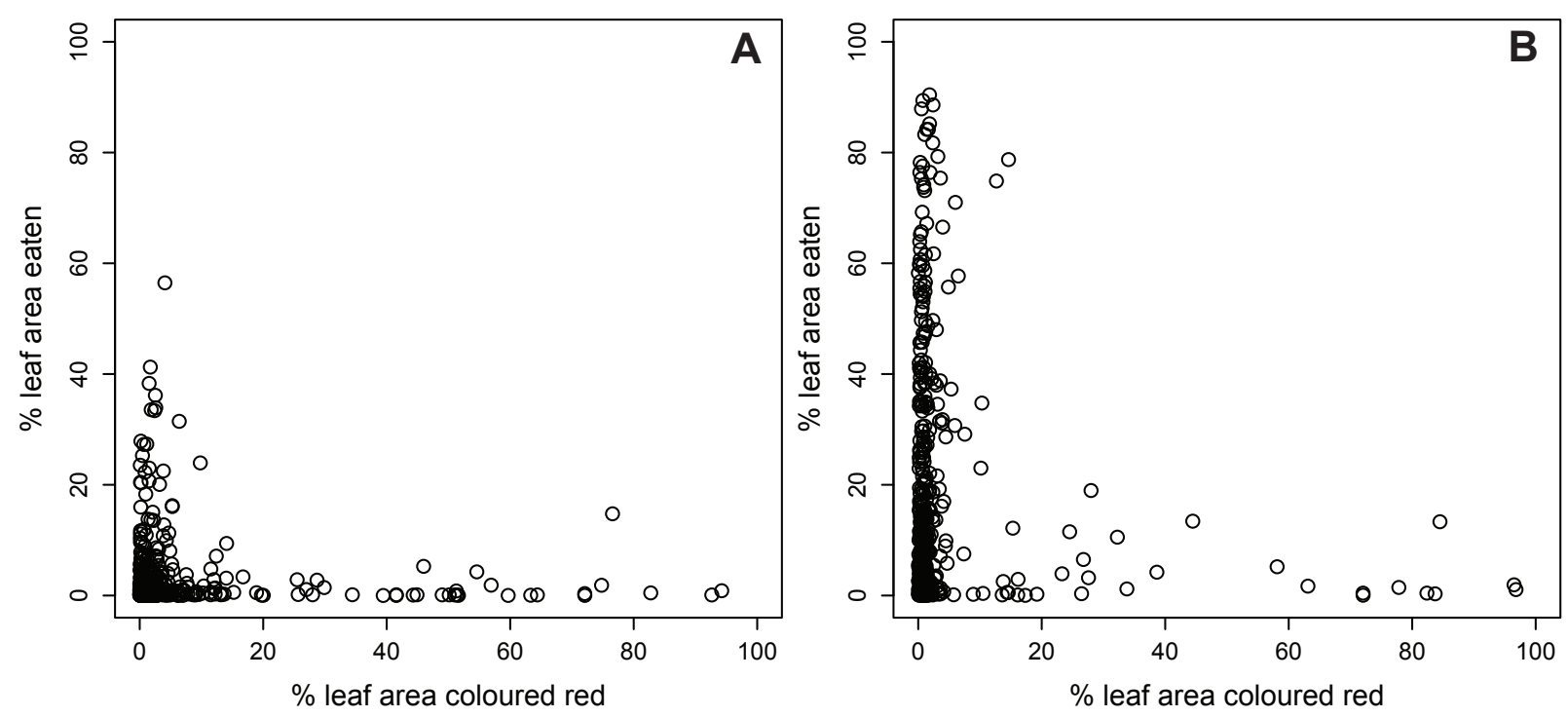

Fig. 2.9 Feeding damage and proportionate red leaf area in leaves of P.colorata from (A) Wellington $(\mathrm{n}=551)$ and $(\mathbf{B})$ Otago $(\mathrm{n}=375)$. Untransformed data presented here, but analyses were conducted on arcsine square root-transformed feeding damage data.

Of the leaves collected for chemical analyses from the Wellington site, polygodial concentration was a better predictor of herbivory than anthocyanin (Table 2.1) and there was no significant interaction between anthocyanin and polygodial. This indicates that anthocyanins are not likely to have direct effects on leaf palatability, and therefore leaf damage, in P. colorata. Of the leaves collected for chemical analyses from the Otago site, neither anthocyanin or polygodial were significant predictors of herbivory, and the interaction term was not significant. The Otago result is unsurprising perhaps, given the low sample size ( $\mathrm{n}=4$ independent individuals) combined with high variability in herbivory.

\subsubsection{Red trees experience less insect pressure than neighbouring green trees}

When matched pairs of $P$. colorata trees with contrasting leaf colour were compared, the redder trees had significantly lower Ctenopseustis spp. leafroller caterpillar density (Wilcoxon signed ranks, $\mathrm{n}=30, \mathrm{Z}=-2.5, P=0.013$, Table 2.2) than did neighbouring green trees. Caterpillar densities upon $P$. colorata trees varied substantially, ranging from $1.4 \mathrm{~m}^{-2}$ to $130.3 \mathrm{~m}^{-2}$. After normalising to control for 
differences in caterpillar densities across pairs, red trees had on average $\sim 22 \%$ fewer caterpillars than green trees. While the redder trees hosted fewer caterpillars, no tree was free of leafroller caterpillars. At the beginning of the season, before the caterpillar census, there was no difference in the proportion of leaves showing signs of herbivory per tree within pairs (paired Student's $t$-test, $\mathrm{n}=30, t=-0.557, P=0.582$ ).

\subsubsection{Neighbouring red and green trees do not differ in fitness}

No differences in the total numbers of fruit per $\mathrm{m}^{2}$ canopy area were observed between matched red and green $P$. colorata trees (Wilcoxon signed ranks, $\mathrm{n}=30, \mathrm{Z}=$ $-0.06, P=0.951$, Table 2.2). Similarly, there were no differences between matched red and green trees in mean numbers of seeds per fruit (paired Student's $t$-test, $\mathrm{n}=26$, $t=-1.3, P=0.213$, Table 2.2), mean seed weight $(\mathrm{n}=26, t=-1.5, P=0.136)$, estimated total number of seeds per $\mathrm{m}^{2}$ canopy area $(\mathrm{n}=26, \mathrm{Z}=-0.24, P=0.809)$, nor estimated total seed mass per $\mathrm{m}^{2}$ canopy area $(\mathrm{n}=26, \mathrm{Z}=-0.47, P=0.638$, Table 2.2).

Table 2.2 Herbivore pressure and fitness parameters of matched pairs of red and green P. colorata trees. Red trees hosted fewer leafroller caterpillars than neighbouring green trees, but there were no statistical differences in the numbers of fruits or seeds, or seed mass per tree. Asterisk indicates statistical significance (Wilcoxon signed ranks [indicated by Z] or paired Student's $t$-test, depending on the outcome of Shapiro-Wilk test for normality, $P<0.05)$.

\begin{tabular}{|c|c|c|c|c|c|c|c|}
\hline & & Mean & Median & S.D. & $\mathrm{N}$ & $\mathrm{Z}$ or $t$ & $P$ \\
\hline \multirow{2}{*}{$\begin{array}{c}\text { No. leafroller } \\
\text { caterpillars per } \mathrm{m}^{2} \\
\text { canopy area }\end{array}$} & Red & 20.3 & 11.2 & 27.1 & \multirow[b]{2}{*}{30} & & \multirow[b]{2}{*}{ 0.013* } \\
\hline & Green & 26.0 & 16.1 & 25.5 & & $\mathrm{Z}$ & \\
\hline \multirow{2}{*}{$\begin{array}{l}\text { No. fruits per } \mathrm{m}^{2} \\
\text { canopy area }\end{array}$} & Red & 271.8 & 131.3 & 316.4 & \multirow{2}{*}{30} & -0.06 & \multirow{2}{*}{0.951} \\
\hline & Green & 236.7 & 167.1 & 291.0 & & $\mathrm{Z}$ & \\
\hline \multirow{2}{*}{ No. seeds per fruit } & Red & 4.2 & 4.1 & 0.8 & \multirow{2}{*}{26} & -1.3 & \multirow{2}{*}{0.213} \\
\hline & Green & 3.9 & 4.1 & 0.7 & & $t$ & \\
\hline \multirow{2}{*}{$\begin{array}{l}\text { Seed mass (mg) per } \\
\mathrm{m}^{2} \text { canopy area }\end{array}$} & Red & 2.4 & 1.6 & 2.4 & \multirow{2}{*}{26} & -0.47 & \multirow{2}{*}{0.638} \\
\hline & Green & 2.1 & 1.1 & 2.8 & & Z & \\
\hline
\end{tabular}




\subsection{DISCUSSION}

Three of my results indicate that red $P$. colorata leaves signal to herbivores: (i) leaves with a higher proportion of red colouration contained greater levels of the antifeedant, polygodial (Fig. 2.8); (ii) there was a marked reduction in the mean amount and variability of herbivory incurred by leaves with greater amounts of red colouration (Fig 2.9); and (iii) red trees hosted fewer herbivorous insects than neighbouring green trees (Table 2.2). However, there was no evidence to suggest that leaf signalling influences the fitness of $P$. colorata. Consequently, we failed to support a central assumption of the leaf signalling hypothesis that signalling plant defences conveys an adaptive benefit.

Equal fitness pay-offs in the face of differing herbivore pressure indicate that variation in leaf colour between $P$. colorata individuals may be a stable polymorphism. Indeed, the widespread occurrence and maintenance of variation in leaf colour among individuals suggests that colour variation represents a stable polymorphism. In light of the equal fitness results, it is not possible to determine if red leaf colour functions either as a cue or a signal in $P$. colorata. Potential fitness benefits of red pigmentation in relation to reduced herbivory may be offset by (unknown) disadvantages associated with red leaf production. There are several scenarios, that are not mutually exclusive, which could result in equal fitness and which are consistent with leaf signalling. These are: (1) there are costs associated with the production of red leaves that in some situations may limit the fitness of red trees; (2) signalling may be part of a compensatory strategy to allow nutrient deficient plants to achieve equal fitness with non-deficient conspecifics; (3) green trees may have evolved mechanisms to tolerate higher levels of herbivory damage or alternative mechanisms to defend from herbivory and (4) fitness impacts of red leaf colour may be obscured by multitrophic interactions. To assess the usefulness of the leaf signalling hypothesis we need to test the relative contribution of each scenario to plant fitness via experimental tests manipulating the presence of herbivores and levels of environmental stress. I outline the four scenarios below. 


\subsubsection{Signalling costs in the absence of herbivores}

Equal fitness in the presence of unequal herbivore damage is explicable if there are significant negative side effects associated with investing in a red leaf signal in the absence of strong herbivore pressure. The signalling hypothesis proposed that red colouration was expensive and thus a handicap (Hamilton and Brown 2001). The main assumption of handicap signalling is that the costs are more difficult to pay for lower quality individuals and therefore the reddest individuals get the highest pay offs. The apparent costs of signalling depend of the level of herbivore pressure. In the absence of herbivores, red leaf signalling trees still face investment costs, yet do not receive fitness pay-offs.

We can expect there to be costs associated with producing the red leaf signal in $P$. colorata. The metabolic costs of manufacturing anthocyanins are small, requiring only a amount of carbon that can be assimilated over a few hours of photosynthesis (Foyer et al. 2007). There are additional (unknown) costs associated with the production of biosynthetic enzymes and with the transport of anthocyanins into the vacuole. Again, these are likely to be trivial, given that they are a one-time investment that lasts for the entire life of a leaf. However, effective deterrence also requires the manufacture, transport, storage and maintenance of higher levels of polygodial (on average, $59 \mathrm{mg} \mathrm{g}^{-1} \mathrm{DW}$ for red leaves versus $34 \mathrm{mg} \mathrm{g}^{-1}$ for green leaves in Wellington; $31 \mathrm{mg} \mathrm{g}^{-1} \mathrm{DW}$ versus $11 \mathrm{mg} \mathrm{g}^{-1}$ in Otago) in signalling leaves.

Additionally, there may be indirect costs of signalling. Due to the light-screening effects of anthocyanins, considerably less green light strikes the chloroplasts of red than of green leaves (Gould et al. 1995, Neill and Gould 1999). Green light is important for photosynthesis, particularly in the lower spongy mesophyll layers of a leaf (Nishio 2000). As a consequence, quantum yields and maximum $\mathrm{CO}_{2}$ assimilation rates can be lower in red than in green leaves (Gould et al. 2002b, Kyparissis et al. 2007, Lan et al. 2011, Nielsen and Simonsen 2011, Nikiforou et al. 2011, Zhang et al. 2011). This may limit the resources available for allocation towards reproduction. Losses in photosynthetic achievement can ultimately result in impaired fitness (Ganeteg et al. 2004). If the levels of herbivore pressure are low then the direct and indirect costs of investing in a signal may result in equal fitness payoffs 
for signalling and non-signalling plants. In the absence of herbivores altogether, one might expect signalling plants to be at a disadvantage.

During this study I found an average difference of $\sim 5$ fewer caterpillars $(\sim 22 \%$ reduction) per $\mathrm{m}^{2}$ canopy area between my matched pairs of green- and red-leafed plants. Additionally, the correlation between herbivory and leaf colour (although statistically significant) was weak, with only $3.5 \%$ of variation in herbivory explained by leaf colour. Studies of autumn leaves have found that variability in non-green leaf colour explained more than $80 \%$ of the variability in the numbers of herbivores (Archetti and Leather 2005) and more than $25 \%$ of the variation in the amounts of insect damage (Hagen et al. 2003). Given that the fitness payoffs associated with signalling are likely to be dependent on the degree of herbivore pressure, the small difference in the number of herbivores between red and green trees in this study may explain why differences in fitness were not observed. There was stronger herbivory pressure at the Otago site, where leaves incurred greater amounts of feeding damage than at Wellington (Fig 2.9). Correspondingly, I also expect to find greater differences in fitness between red and green $P$. colorata at Otago.

\subsubsection{Signalling may be part of a compensatory strategy for nutrient deficient plants}

$P$. colorata leaves from both populations exhibited a very large range of leaf colouration, far beyond the amount needed to significantly reduce variation in herbivory (i.e., $4.25 \%$ leaf area coloured red for leaves from Wellington and $4 \%$ for those from Otago, Fig 2.9). Similarly, Cooney et al. (2012) found that only a thin band of red colouration around the leaf margin $(1.75-2.25 \%$ of total leaf width) was needed to be an effective deterrent, yet much wider red margins were common. Such a large range of leaf colouration suggests that anthocyanin pigments may be carrying out other functions within $P$. colorata leaves. Redness above the threshold for reduced herbivory predominantly occurred in the uppermost, exposed leaves of the canopy. Another polyphenol, chlorogenic acid, known to be a powerful antioxidant (Grace et al. 1998, Close and McArthur 2002), was found with a similar distribution to anthocyanins within P. colorata leaves (Fig. 2.4C). The high level of antioxidant compounds in these upper leaves suggests that they are prone to photo-oxidative stress. 
Recent research on transient winter-reddening in two Mediterranean species, Cristus creticus and Pistacia lentiscus, may provide insight into these results. During winter, some individuals of these species turn red while neighbouring conspecifics remain green. Researchers assessed photosynthetic and photoprotective parameters in red and green phenotypes of both species. They concluded that red phenotypes were characterised by lower photosynthetic efficiency, lower levels of xanthophyll cycle pigments and lower leaf nitrogen, and were more susceptible to photoinhibition than neighbouring green individuals (Kytridis et al. 2008, Zeliou et al. 2009, Nikiforou et al. 2010, Nikiforou et al. 2011). Similar results have been seen for winter-red phenotypes of Japanese Honeysuckle Lonicera japonica (N. Hughes pers. comm.) and in the red young leaves of Rosa sp. and Ricinus communis L. (Manetas et al. 2002). Anthocyanin pigments in red-leaf $C$. creticus were demonstrated to have a photoprotective role both by shading subjacent chloroplasts (Kytridis et al. 2008) and through antioxidant activity (Kytridis and Manetas 2006). Red phenotype Coleus spp. maintain similar photosynthetic light use as green phenotypes (B. A. Logan pers. comm.). In contrast, the physiological benefits of anthocyanin accumulation in the winter-reddening Cristus creticus were not sufficient to compensate for the higher vulnerability of red phenotype to photoinhibition (Kytridis et al. 2008, Zeliou et al. 2009, Nikiforou et al. 2011). Despite this, Nikiforou et al. (2010) found no difference in relative fitness of red and green individuals of $C$. creticus, similar to my study. Perhaps nutrient-limited or genetically inferior P. colorata and C. creticus individuals accumulate anthocyanin pigments in their leaves for the combined benefits of both signalling and photoprotection. Anthocyanin pigments are predominatly located within the epidermal cells of $P$. colorata leaves (Fig. 2.1F). This is consistent with both light screen and signalling functions. The dual functions of anthocyanins in this scenario may allow weak individuals to maintain equal fitness to their neighbouring conspecifics.

After herbivore damage anthocyanin pigments may also play other additional roles within leaves. Zangerl et al. (2002) and Nabity et al. (2009) found a significant reduction in the photosynthetic rate of intact tissue near the site of herbivore damage, which may lead to increased chances of photo-oxidative damage. Additionally, the release of autotoxic defence compounds into leaf lamina after herbivore damage has 
been demonstrated to cause oxidative damage (Gog et al. 2005). The antioxidant function of anthocyanins around the site of damage has already been demonstrated for P. colorata (Gould et al. 2002a). Chapter 4 contains results of investigations into photosynthesis of red and green $P$. colorata. However the nutrient status and xanthophyll pools and of red and green $P$. colorata at Wellington have not been quantified.

\subsubsection{Tolerance vs. resistance to herbivores}

To date, discussions about the evolution of plant-animal signalling have focused on chemical defences that provide resistance to herbivores. However, in recent years there has been greater recognition of the role of plant tolerance to herbivory (Strauss and Agrawal 1999, Fornoni 2011). Diverse mechanisms of tolerance allow plants to mitigate the negative effects of herbivory on fitness, such as increased photosynthetic rate, increased shoot growth rate, and reallocation of carbon stores from roots to shoots after damage (Stowe et al. 2000). In some species, an individual may employ both resistance and tolerance mechanisms (Salgado-Luarte and Gianoli 2010) and there can be inter-individual differences in patterns of allocation to these two defence strategies (Leimu and Koricheva 2006, Núñez-Farfán et al. 2007). It is plausible that, once a signalling/chemical resistance system evolves, the increased herbivory experienced by non-signalling individuals may lead to increased selection for tolerance mechanisms.

Leaf colouration may reveal an individual's pattern of allocation to tolerance and resistance defence strategies. Redder individuals would invest more resources in resisting herbivores; these investments return benefits in terms of fewer caterpillars and smaller amounts of feeding damage, yet also incur indirect costs. In contrast, greener individuals would use a defence strategy biased towards tolerance, investing fewer resources to resisting herbivores. Instead, these resources would be used for increased and faster regrowth in green individuals. Equal fitness pay-offs suggest that the two strategies would be stable within populations, explaining the maintenance of leaf colour variation. I did not collect growth rate data for P. colorata; however, whilst not directly testing for tolerance mechanisms, Nikiforou et al. (2010) found higher numbers of leaves per unit shoot length in green as compared with red $C$. 
cristus plants. However, in the same study, red plants showed higher stem elongation rate. While more data are needed, clearly experimental and theoretical studies of red leaf signalling need to include tolerance in their discussions of plant defence.

\subsubsection{Tritrophic and indirect interactions}

Since these plants and their herbivores are parts of an integrated ecosystem there may be wider ecological effects that influence plant fitness. Red pigments in the leaves of P. colorata may function for signalling, but the beneficial fitness effects of signalling may be obscured by tritrophic interactions. The negative effect of high numbers of caterpillars on green trees may be nullified if green trees have higher levels of predation and parasitism, and therefore reduced survival of insect herbivores. Predators and parasitoids of insect herbivores have been repeatedly shown to use volatile organic compounds (VOC) emitted from leaves after insect feeding damage as cues to locate their prey (Turlings et al. 1990, Kessler and Baldwin 2001, Allison and Hare 2009, Hare 2011). It has been demonstrated that yellow and green autumn leaves differ in their VOC profiles (Holopainen 2008, Holopainen et al. 2010). Therefore it may also be important to look at plant volatiles in the context of $P$. colorata's red and green leaf polymorphism (see Chapter 3).

Green $P$. colorata trees may experience higher predation rates upon their herbivores through another mechanism. Shelter-building species, such as leafroller caterpillars, are considered ecosystem engineers: after the caterpillars emerge as imagos, the rolled- or webbed-together leaves provide a habitat resource, which increases arthropod biodiversity (Martinsen et al. 2000). Manipulative experiments have repeatedly found that leaf shelters can increase the abundance of predatory ants, beetles and spiders (Martinsen et al. 2000, Fournier et al. 2003, Nakamura and Ohgushi 2003). The indirect effects of leaf shelters can affect the whole community of herbivores upon trees (Fukui 2001, Nakamura and Ohgushi 2003, Ohgushi 2005). It was beyond the scope of this study to survey predators and parasitoids, however I did note several occurrences of observing spiders in vacant $P$. colorata leaf shelters.

\subsubsection{Other considerations}

My conclusions regarding plant fitness are based upon estimates of fitness, derived 
from inter-individual differences in fitness components. As such, these conclusions rely on the untested assumption that individual plants that produce the greater number of seeds, contribute a greater number of surviving offspring to the subsequent generations (Primack and Kang 1989, McGraw and Caswell 1996). Additionally, there may be interactions between fitness components that influence ultimate fitness (Strauss and Agrawal 1999, Stowe et al. 2000). Despite these critiques, the majority of studies of plant defences use seed production as a proxy for fitness (Strauss and Agrawal 1999). Given the time constraints of a $\mathrm{PhD}$, my measures of reproductive effort were the most practical and best available.

Patterns of leaf colouration, defence and herbivory were studied from two different populations of $P$. colorata. Even though the two populations are located in very different environments (forest sub-canopy vs. exposed hilltop canopy) the relationships between leaf colouration, defence and herbivory within each population were consistent. Redder leaves tended to contain higher concentrations of the defence compound polygodial, and experienced reduced amounts and variability of insect herbivory (Fig $2.8 \&$ Fig 2.9). These results are in agreement with another study using red leaf margins in P. colorata (Cooney et al. 2012).

The observed correlation between anthocyanins and polygodial is surprising given that the flavonoid and terpenoid biosynthetic pathways are distinct. Insertion of the Arabidopsis PRODUCTION OF ANTHOCYANIN PIGMENT 1 (PAP1) transcription factor into Rosa hybrid caused, along with increased colour, an increase in the release of terpenoid volatile organic compounds, indicating the possibility of crosstalk between these two pathways (Ben Zvi et al. 2012). Additionally, anthocyanin pigments in petals have been demonstrated to correlate with induced levels of defensive glucosinolate compounds in leaves of Raphanis sativus (Strauss et al. 2004). This was thought to result from either tight linkage of, or pleiotropic effects between, pigment and defence genes (Fineblum and Rausher 1997, Strauss et al. 2004).

Finally, a correlation between polygodial and anthocyanin levels within P. colorata leaves may partially be caused by an interaction between leaf ontogeny and exposure to environmental stressors. However, it is worth noting that while young $P$. colorata 
leaves were redder and contained high levels of polygodial, there was no age-related pattern in the levels of the closely related dialdehyde, 9-deoxymuzigadial, in either population (Fig. 2.5B \& Fig. 2.5B). 


\section{Volatile profiles of red and green $P$. colorata leaves}

\section{$3.1 \quad$ ABSTRACT}

Foliar anthocyanins might be effective visual signals to deter herbivores. Given that many insects are sensitive to volatile organic compounds (VOCs), a plant might be expected to use both visual and olfactory cues. However the role of VOCs in plantherbivore visual signalling has not been investigated. I analysed VOCs released from undamaged, herbivore- and mechanically-damaged red and green leaves of Pseudowintera colorata, and the olfactory preferences of brownheaded leafroller (Ctenopseustis obliquana) larvae. I tested the hypotheses that (i) VOC profiles of $P$. colorata leaves are a reliable indicator of the defensive potential of the leaf, and correlate with anthocyanin content; (ii) leaves with greater red coloration emit a greater amount of sesquiterpenes in their VOC profiles; (iii) any insect deterrent compounds will be released in greater amounts by the red than the green leaves; and (iv) both naïve and experienced C. obliquana larvae will prefer the VOCs released from green $P$. colorata leaves. While the VOC profiles of browsed and unbrowsed leaves were statistically distinguishable, the VOC profiles released from intact, herbivore-, and mechanically-damaged $P$. colorata leaves did not reliably identify leaf colour. Mechanically damaged red $P$. colorata leaves did not release statistically significant higher levels of sesquiterpenes than damaged green leaves. I did not identify any compounds known to be strong deterrents, such as methyl salicylate or farnesene. Moreover, naïve and experienced C. obliquana larvae displayed no preference for the volatiles from mechanically damaged red or green leaves. Therefore, I conclude that VOC compounds are not likely to play a large role in mediating insect herbivore-plant interactions in P. colorata.

\subsection{INTRODUCTION}

Anthocyanin pigments and an assortment of volatile organic compounds (VOCs) have been postulated to protect plant vegetative tissues from biotic and abiotic stressors (Steyn et al. 2002, Close and Beadle 2003, Gould 2004, Dudareva et al. 2006, Vickers et al. 2009). However, research into the functional significance of these two types of plant metabolites has progressed largely independently of each other. It has been 
hypothesised that anthocyanins may function as visual signals to deter approaching insect herbivores (Archetti 2000, Hamilton and Brown 2001). Several studies have found correlations between non-green leaf colour and reduced insect herbivore pressure in the field, and have interpreted these as support for pigment-mediated visual signalling (Hagen et al. 2003, Archetti and Leather 2005, Karageorgou and Manetas 2006, Wong and Srivastava 2011, Cooney et al. 2012). In those studies the possible effects of plant volatiles on insect deterrence were not considered, yet it has been repeatedly proposed that leaf colour may correlate with leaf VOC emissions (Archetti and Brown 2004, Ougham et al. 2005b, Holopainen 2008, Döring et al. 2009, Lev-Yadun and Gould 2009). Indeed, only one study to date has investigated VOCs in differently coloured leaves; Holopainen et al. (2010) found that green nonsenescing leaves emitted different VOC profiles than yellow senescing leaves, and concluded that the green leaves were potentially the better defended against insect herbivores. However, non-green leaf colour is extremely widespread and is not restricted to senescing leaves (Lee 2002). To my knowledge, no study has investigated the VOC profiles from anthocyanic non-senescing leaves.

Although anthocyanins might be effective visual signals (see Chapter 2), they are themselves not volatile compounds, and therefore would not be expected to deter herbivores that have only poor colour vision, or nocturnal herbivores. Because foliar anthocyanins are stored in cell vacuoles, the production of a red leaf signal, requiring a one-time investment that potentially lasts the entire life of a leaf, may not be very costly. In contrast, olfactory signalling via VOCs would be effective day and night, yet would require continuous production of metabolically expensive compounds which, once emitted, are irretrievably lost to the atmosphere (Vickers et al. 2009). To most efficiently maximise the chances of successful defence against herbivory, a plant might therefore be expected to use both visual and nocturnal olfactory cues.

In comparison to the number of studies investigating the influence of VOCs on the behaviour of adult Lepidoptera, the olfactory preferences of lepidopteran larvae have been largely overlooked (Carroll et al. 2006). Some lepidopteran larvae are very mobile and can disperse through crawling onto nearby more-preferable hosts (Zaluki et al. 2002). Olfactory cues may be used in these larval voyages (Landolt et al. 2000, Carroll et al. 2006, Perkins et al. 2008). For example, the larvae of the European corn 
borer display innate olfactory preferences towards higher quality host species (Piesik et al. 2009). Experience of plants can modify subsequent insect host preferences (Pszczolkowski and Brown 2005), which can be carried through metamorphosis into adults (Blackiston et al. 2008). Additionally lepidopteran larvae have been shown to learn to associate odours with specific tastes (Salloum et al. 2011). P. colorata trees with redder leaves hosted fewer leafroller caterpillars than their greener neighbouring trees (see Chapter 2). If red and green P. colorata do differ in their VOC profiles this recorded difference in nocturnal foraging leafroller larvae could be attributable to olfactory cues.

Traditionally, studies on the role of VOCs in plant defence have focused on VOCs produced only after herbivore attacks, termed 'herbivore-induced plant volatiles' (HIPVs). Herbivore feeding, even by below-ground herbivores (Soler et al. 2007), causes the synthesis and release of specific mixes of VOCs from leaves (Turlings et al. 1998, Huber and Bohlmann 2004, Dudareva et al. 2006). These HIPVs function as direct defences and have been shown to deter a diverse range of herbivores including lepidopteran adults and larvae (De Moraes et al. 2001, Kessler and Baldwin 2001, Laothawornkitkul et al. 2008), thrips (Delphia et al. 2007) and aphids (Bernasconi et al. 1998, Vancanneyt et al. 2001, Aharoni et al. 2003). In addition, HIPVs are used by predators and parasitoids as cues to locate their herbivore prey (Dudareva et al. 2006, Mäntylä et al. 2008, Unsicker et al. 2009, Hare 2011). Variation in HIPVs has been documented between individual plants in the field (Gouinguene et al. 2001, Hare 2007, Delphia et al. 2009, Schuman et al. 2009).

A swifter method of defence might be achieved if the tissue damaged by insect feeding were to immediately release a potent burst of deterrent VOCs. Several species of plants store pools of volatile terpenoids in specialised structures in their vegetative tissues (Loughrin et al. 1994, Paré and Tumlinson 1997, Franceschi et al. 2005, Keeling and Bohlmann 2006). These stored terpenoids, after release by insect feeding, are effective at deterring herbivores (Keeling and Bohlmann 2006). P. colorata tissues are constitutively defended by sesquiterpene dialdehydes (Gerard et al. 1993) stored in idioblast cells (Youard 2012). P. colorata leaves with higher levels of anthocyanin pigments have been shown to contain higher levels of these chemical defences (see Chapter 2). Within leaves, anthocyanic regions have been shown to 
contain higher levels of polygodial (Cooney et al. 2012). While the sesquiterpene dialdehydes are not especially volatile at room temperature (C. Sansom pers. comm.), other more-volatile sesquiterpenes may also occur within idioblasts. Thus, we might expect differences in the VOC profiles of damaged red and green leaves as a result of these differences in chemical defences.

Damage to plant cells causes the release of a typical suite of fatty acid lipoxygenase products consisting of six-carbon aldehydes, alcohols and esters (Hatanaka 1993, Turlings et al. 1998, Holopainen 2004, D'Auria et al. 2007, Allmann and Baldwin 2010). Because of their distinctive 'freshly cut grass' smell, these fatty acid derivatives are referred to green leaf volatiles (GLVs). GLVs are ubiquitous in the plant kingdom, and are detected by insect olfactory receptor neurons (ORNs) with extremely high levels of sensitivity (Bruce et al. 2005, Bruce and Pickett 2011). ORNs specifically for ubiquitous GLV compounds are found throughout herbivorous insects (Bruce et al. 2005, Bruce and Pickett 2011, Wei and Kang 2011), and have been recorded on the maxillary palps of lepidopteran larvae (Roessingh et al. 2007). Different studies report GLV compounds acting as repellents or attractants (Natale et al. 2003, Huang et al. 2009, Grant et al. 2010). GLVs are clearly key infochemicals, used by herbivorous insects in host-selection decisions. Moreover, GLVs have been shown to reduce fitness of herbivores such as aphids (Hildebrand et al. 1993, Vancanneyt et al. 2001) and spider mites (Dicke and Dijkman 2001).

I analysed volatiles released from intact, herbivore damaged, and mechanically damaged red and green $P$. colorata leaves to test the following hypotheses: (i) the VOC profiles of $P$. colorata leaves are a reliable indicator of the defensive potential of the leaf, and correlate with anthocyanin content; (ii) leaves with greater red coloration emit a greater amount of sesquiterpenes in their VOC profiles; and (iii) any insect deterrent compounds will be released in greater amounts by the red than the green leaves. Additionally, I tested if brownheaded leafroller (Ctenopseustis obliquana) larvae display preferences for VOCs emitted from damaged red and green $P$. colorata leaves using a Y-tube olfactometer. Naïve, lab-reared larvae were assayed, given experience of red and green $P$. colorata leaves and re-tested. I hypothesized that (iv) both naïve and experienced $C$. obliquana larvae will prefer the VOCs released from green $P$. colorata leaves. 


\subsection{MATERIALS AND METHODS}

\subsubsection{Plant material}

VOC profiles were collected from a natural population of $P$. colorata located at Belmont trig, Wellington, New Zealand $\left(41^{\circ} 11^{\prime} 0.2^{\prime \prime} \mathrm{S}, 174^{\circ} 52^{\prime} 25.9^{\prime \prime} \mathrm{E}\right)$. Leaves from this population were also used for mechanical damage and Y-tube experiments. At Belmont trig $P$. colorata forms a dense thicket on an exposed hilltop which received direct sunlight throughout the day. HIPV emissions were measured on leaves from potted nursery-grown $P$. colorata plants ecosourced from forests near Dunedin, Otago.

\subsubsection{Insects}

Brown-headed leafroller larvae, Ctenopseustis obliquana (Walker) (Lep. Tortricidae), were obtained from a lab colony at Plant \& Food Research Limited in Auckland, NZ, and maintained at $20{ }^{\circ} \mathrm{C}, \sim 60 \%$ relative humidity and 16:8 (light:dark) photoperiod. Larvae were reared individually in $5 \mathrm{~mL}$ test-tubes with cotton-wool caps, containing an artificial diet (Singh 1983). To ensure larvae were hungry, they were transferred to test-tubes lacking the artificial diet $6 \mathrm{~h}$ before bioassays.

\subsubsection{Plant volatile collection in the field}

Field VOCs were collected in December (late spring) 2012. I selected three pairs of $P$. colorata trees at the Wellington site following the methods of Hagen et al. (2004). Each pair contained one tree whose leaves were predominantly green and one tree whose leaves were much redder. The individuals in each pair were located no more than $2 \mathrm{~m}$ apart from one another, and were closely matched in size, exposure to direct sunlight and prevailing winds. This design allowed me to investigate the effect of leaf colour on VOC emission whilst minimising differences in environmental conditions between red and green plants within a pair. One stem was selected from the periphery of the canopy of each individual in the three pairs. Within pairs, the stems were closely matched for azimuth, numbers of leaves, exposure to direct sunlight and prevailing winds. Stems with any evidence of caterpillars or flowers were excluded. A polyethylene terephthalate oven bag (Glad "extra large" 500 x 500mm, Clorox 
Australia Pty Limited, Australia) was placed over each selected stem and the opening carefully fastened to the stem with a twist-tie (after Holopainen et al., 2010). One corner of the oven bag was cut to make a hole just large enough to insert a solid-phase microextraction (SPME) fibre-holder (Supelco, Shanghai, China). The oven bag was twist-tied securely to the holder and a pre-conditioned $65 \mu \mathrm{m}$ polydimethylsiloxane/divinylbenzene SPME fibre (Supelco, Shanghai, China) was inserted in to the holder, but not exposed to the air inside the bag (Fig. 3.1). A second oven bag was placed over the entire SPME fibre and holder apparatus, and clamped in place.

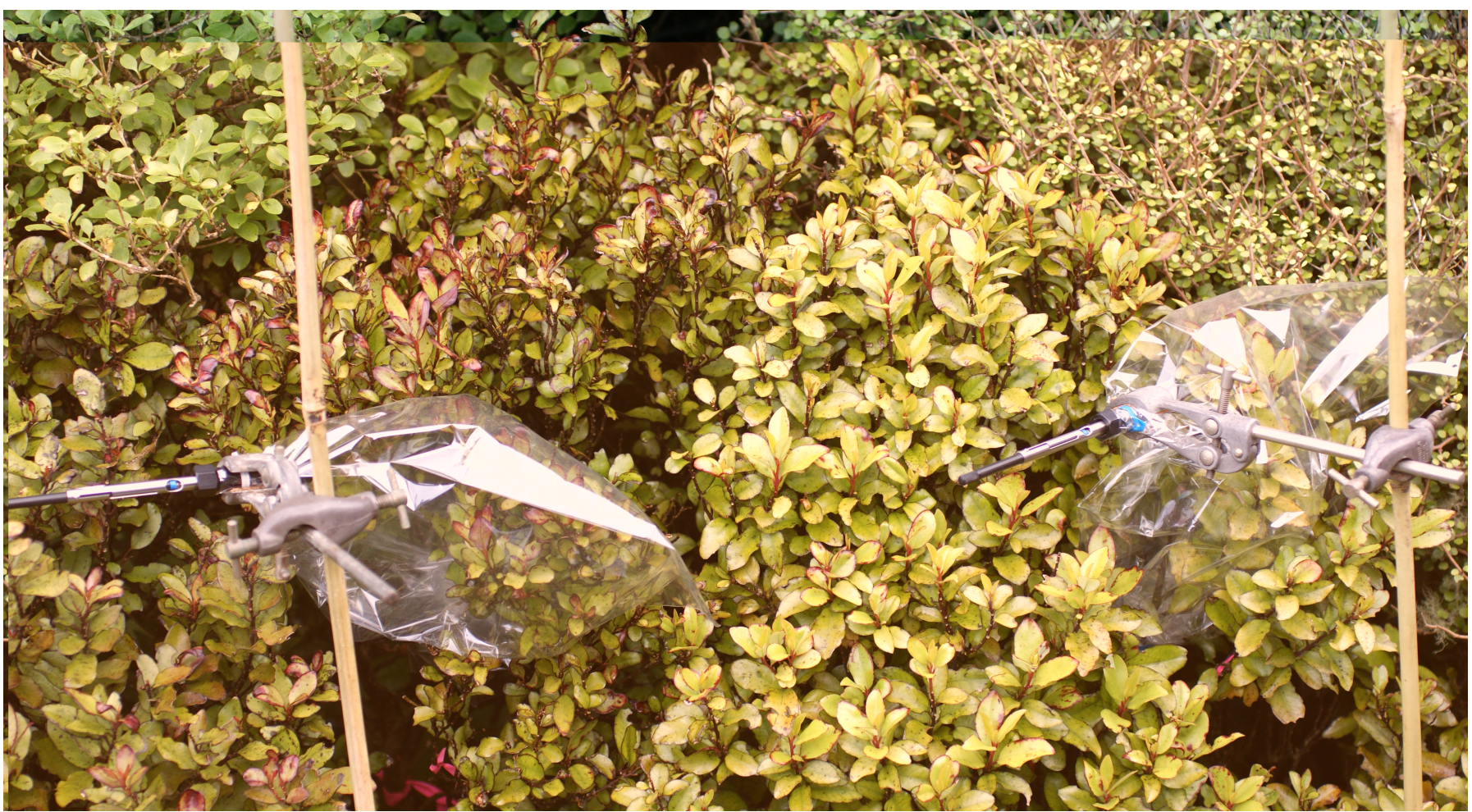

Fig. 3.1 Oven bags and SPME fibres and holders used to collect VOCs from $P$.

colorata plants in the field.

In the early evening, after each pair's oven bag and holder setup was complete, the fibres were exposed (allowing VOC collection) simultaneously. Seven h later, when the wind became very strong, each apparatus were dismantled in the same sequence as they had been set up.

SPME fibres had been conditioned at Plant \& Food Research Ltd in Dunedin, sealed in a glass vial to avoid contamination with polystyrene or other volatile emitting plastics and couriered on ice to Wellington. Upon arrival in Wellington, the fibres 
were kept in a $-80^{\circ} \mathrm{C}$ freezer, then transported to the field site on ice. After VOC collection, the SPME fibres were put back into glass vials on ice, and couriered back to Dunedin the following day. To further avoid contamination I wore no suncream or other volatile-containing body products and took field notes with pencil only.

\subsubsection{Herbivore-induced volatile assay}

Five potted $P$. colorata seedlings were used. As there were not strong differences in colouration among individual seedlings, a pair of leaves was selected from each plant. Each pair consisted of a predominantly red and a predominantly green leaf matched for age (node number) and size. Leaves with signs of previous herbivory were excluded from analysis. Each leaf was placed in $20 \mathrm{~mL}$ glass vial containing a starved caterpillar. As a control, an additional vial contained a caterpillar but no leaf. The gaseous composition inside each vial was analysed using a gas chromatograph-mass spectrophotometer (GC-MS, Agilent Technologies, PA, USA). An SPME fibre was automatically inserted into the first vial and exposed for $60 \mathrm{~min}$ at room temperature then automatically injected into the GC-MS (Fig. 3.2A \& B). The same procedure was used to collect VOCs from each vial sequentially. As a result of the sequential sampling constraint, the leaf in each vial had a slightly longer exposure to its caterpillar, and a longer duration for VOC release, than the previous leaf. Compounds that were strongly present in the caterpillar blank as well as the other vials were determined to be caterpillar related and not included in analyses.

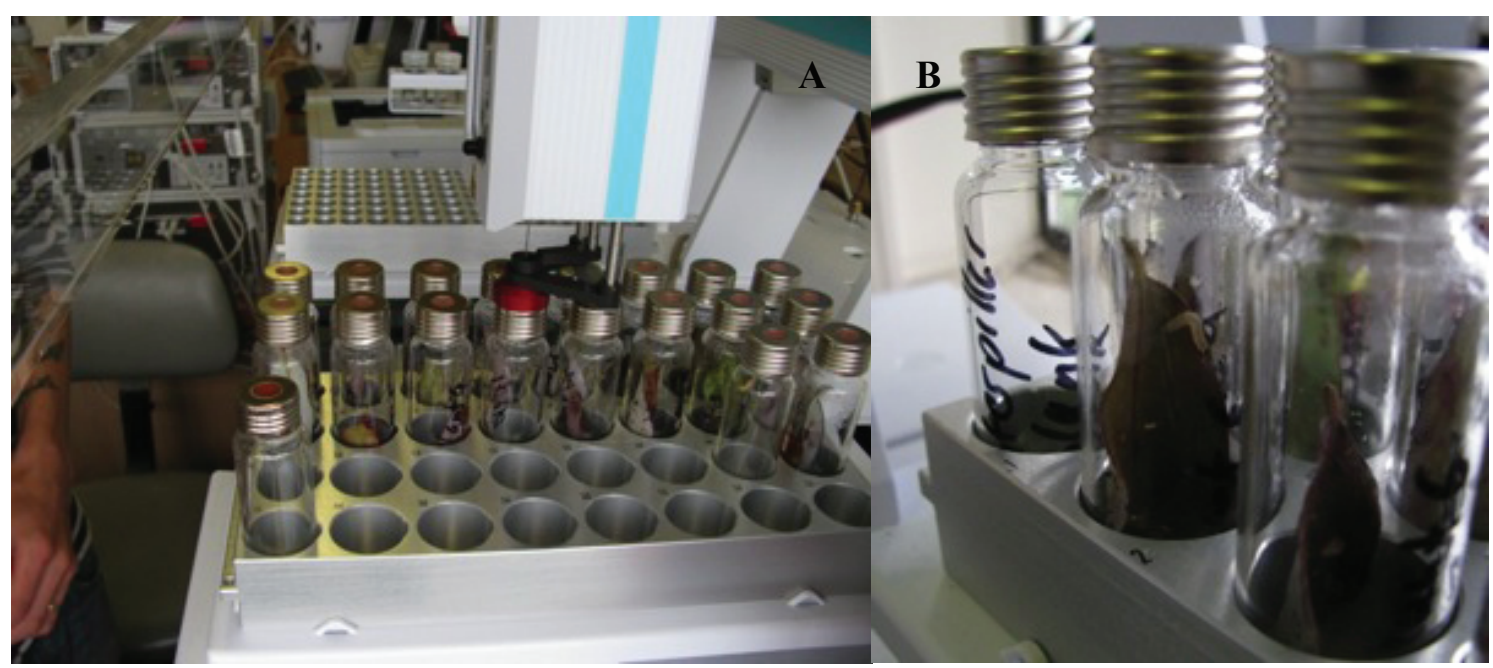

Fig. 3.2 (A) GC-MS autosampler; (B) Leaves and caterpillars in vials. 


\subsubsection{Mechanical injury and plant volatile emissions}

Ten matched pairs of green and red P. colorata from Belmont trig were selected (a random subset of the pairs used in the insect bioassays described below). In March 2012, a small sprig bearing 5 fully expanded leaves was excised from the top of the canopy of each plant. Each stem was re-cut under water and then placed in a $20 \mathrm{~mL}$ florist's tube containing fresh water. In the laboratory, the cut stem was wrapped in moist paper towel and the entire sprig placed within a resealable plastic bag. These bags were couriered overnight to Dunedin on ice within an insulated polystyrene container. In Dunedin one leaf was selected from each sprig of each pair. Leaves were matched as closely as possible within pairs for leaf age (node number) and size. Leaves with extensive mechanical injury or herbivory were excluded. Each selected leaf was excised, swiftly torn into quarters, placed within a clean glass vial, sealed and placed in the autosampler tray. The SPME fibre was immediately exposed to the sample at room temperature for $5 \mathrm{~min}$, before being injected into the GC-MS for analysis.

\subsubsection{SPME}

Static headspace sampling via SPME is a rapid, cost effective and extremely sensitive technique for collecting VOCs (Koziel et al. 1999, Tholl et al. 2006). Plant material is sealed in a container and volatile compounds are collected upon an adsorbent coating of the surface of the SPME fibre exposed to the air within the container. However, different types of coatings or experimental conditions (particularly air temperature and the duration of exposure of fibre to headspace) can dramatically influence the yield of VOCs (Valero et al. 2000, Costa Freitas et al. 2001, IOFI Working Group on Methods of Analysis 2010). Additionally, a build up of humidity and heat within the container may affect plant physiology and VOC emissions (Tholl et al. 2006). I used a general purpose $65 \mu \mathrm{m}$ polydimethylsiloxane/divinylbenzene SPME fibre, frequently used in headspace analysis of plant volatiles (http://www.sigmaaldrich.com/etc/medialib/docs/Supelco/General_Information/1/t19 9925.Par.0001.File.tmp/t199925.pdf, accessed July 2012). Great care was taken to standardise fibre exposure times, and both insect herbivory and mechanical damage experiments were carried out at room temperature. To minimise illumination and 
temperature fluctuations, field volatiles were collected from early evening, into the night.

Each SPME fibre was conditioned at $250{ }^{\circ} \mathrm{C}$ in the $\mathrm{GC}$ injector port for $30 \mathrm{~min}$ before its first use and for $15 \mathrm{~min}$ at the start of each day of sampling.

\subsubsection{GC-MS procedure}

Analyses were performed with the assistance of Catherine Sansom at the University of Otago, using an Agilent 7890A gas chromatograph with a CTC Analytics PAL system autosampler and an Agilent 5975C inert XL MSD with triple axis detector (under the control of Enhanced ChemStation software). Detection was by mass spectrometry (MS).

The three different experiments were analysed using three different GC-MS methods, detailed below.

\subsubsection{Field plant volatiles}

The injector $\left(260^{\circ} \mathrm{C}\right)$ flow was splitless with a purge flow of $50 \mathrm{~mL} / \mathrm{min}$ after $1 \mathrm{~min}$. Manual SPME desorption was for 1 min onto a $30 \mathrm{~m}$ Agilent HP-5ms column with a $0.25 \mathrm{~mm}$ ID and $0.25 \mu \mathrm{m}$ film, split $1: 1$ between FID and MS detection. The carrier gas was hydrogen with a flow of $1.7 \mathrm{~mL} / \mathrm{min}$. The oven was heated from $50^{\circ} \mathrm{C}$ to 250 ${ }^{\circ} \mathrm{C}$ at $5{ }^{\circ} \mathrm{C} / \mathrm{min}$. The MS transfer line was held at $300{ }^{\circ} \mathrm{C}$, the MS source was held at $230{ }^{\circ} \mathrm{C}$ and the MS quad held at $150{ }^{\circ} \mathrm{C}, \mathrm{m} / \mathrm{z} 35-300$.

\subsubsection{Herbivore-induced volatiles}

The injector $\left(300^{\circ} \mathrm{C}\right)$ flow was splitless with a purge flow of $50 \mathrm{~mL} / \mathrm{min}$ after 0.5 min. SPME desorption was for 1 min onto a $30 \mathrm{~m}$ Agilent HP-5ms column with a $0.25 \mathrm{~mm}$ ID and $0.25 \mu \mathrm{m}$ film, split $1: 1$ between FID and MS detection. The carrier gas was hydrogen with a flow of $1.5 \mathrm{~mL} / \mathrm{min}$. The oven was heated from $50{ }^{\circ} \mathrm{C}$ to 60 ${ }^{\circ} \mathrm{C}$ at $1{ }^{\circ} \mathrm{C} / \mathrm{min}$ then to $300{ }^{\circ} \mathrm{C}$ at $30^{\circ} \mathrm{C} / \mathrm{min}$. The MS transfer line was held at $200{ }^{\circ} \mathrm{C}$, the MS source was held at $230{ }^{\circ} \mathrm{C}$ and the MS quad held at $150{ }^{\circ} \mathrm{C}, \mathrm{m} / z$ 35-300.

\subsubsection{Mechanically damaged leaf volatiles}

The injector $\left(260^{\circ} \mathrm{C}\right)$ flow was splitless with a purge flow of $50 \mathrm{~mL} / \mathrm{min}$ after $1 \mathrm{~min}$. SPME desorption was for 1 min onto a $30 \mathrm{~m}$ Agilent HP-5ms column with a $0.25 \mathrm{~mm}$ 
ID and $0.25 \mu \mathrm{m}$ film, split 1:1 between FID and MS detection. The carrier gas was hydrogen with a flow of $1.5 \mathrm{~mL} / \mathrm{min}$. The oven was heated from $40{ }^{\circ} \mathrm{C}$ to $175^{\circ} \mathrm{C}$ at 5 ${ }^{\circ} \mathrm{C} / \mathrm{min}$. The MS transfer line was held at $260{ }^{\circ} \mathrm{C}$, the MS source was held at $230{ }^{\circ} \mathrm{C}$ and the MS quad held at $150{ }^{\circ} \mathrm{C}, \mathrm{m} / \mathrm{z} 35-300$. Peaks were identified by comparing their retention time and mass spectra with those of standards, or by comparing their retention index and mass spectra with those in the Adams, NIST or Plant \& Food Research Ltd libraries. A blank and a series of alkanes were analysed with each batch of samples for calculation of retention indices. Peaks in different samples were aligned using the eluting times of identified known compounds. Compounds for which peaks were greater than $2.5 \%$ of the total ion count were included in statistical analyses. To avoid incorrectly classifying unknown compounds, different nomenclature was used for unidentified peaks in the three different analyses.

\subsubsection{Y-tube olfactometers}

Y-tube olfactometers were constructed from $4 \mathrm{~mm}$ thick clear acrylic. The upper arms of the Y-tubes were $50 \mathrm{~mm}$ long and $120^{\circ}$ apart. Air was filtered and humidified through distilled water and activated charcoal then pumped down each upper arm of the Y-tube at $0.2 \mathrm{~L} / \mathrm{min}$. Air passed through a baffle to distribute airflow evenly over leaf samples, down each arm and out through a hole at the bottom of the Y. Metal mesh prevented larvae from accessing the leaves or the exit hole. The top of each Ytube was removable for insertion of leaf samples and larvae (Fig. 3.3A \& B). 


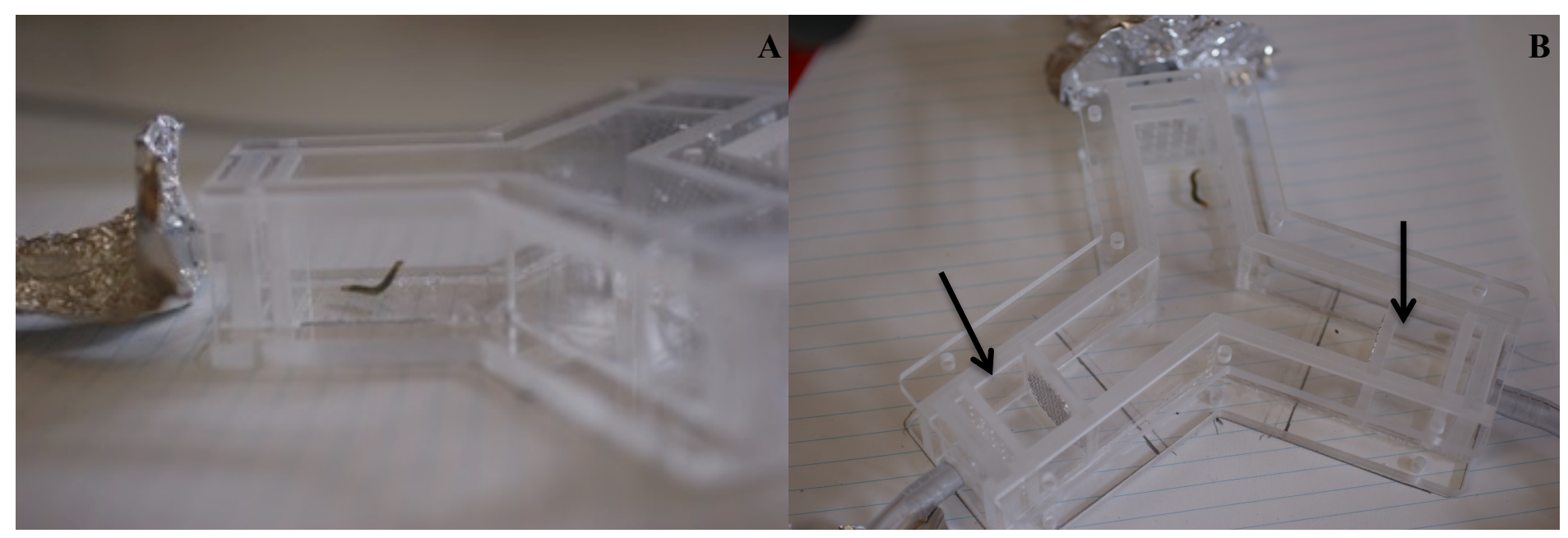

Fig. 3.3 Y-tube olfactometer (A) side view and (B) view from above, both with $C$. obliquana larvae. Arrows indicate where leaf samples were positioned.

\subsubsection{Bioassay protocol}

During March 2012, 30 matched pairs of P. colorata from Belmont trig were selected. A small sprig of 5 leaves was excised from the top of the canopy of each plant. Each stem was re-cut under water and then placed in a $20 \mathrm{~mL}$ florist's tube containing fresh water. Back at the lab, one undamaged leaf was selected from each sprig of each pair. Leaves were matched as closely as possible within pairs for leaf age (node number) and size.

As C. obliquana larvae are nocturnal (http://www.hortnet.co.nz/publications/hortfacts/hf401027.htm, accessed May 2012), bioassays were conducted at night in a dimly lit lab. Starved 5th instar larvae were gently placed in the Y-tube and prevented from travelling up into the upper arms of Y-tube by a cotton wool ball. After around $30 \mathrm{~s}$, matched red and green leaves were excised, swiftly torn into quarters and immediately placed in either arm of the Y-tube, between the baffle and the mesh barrier. Tubes were attached to allow airflow through the Y-tube, the cotton ball was removed and the lid was closed.

C. obliquana larvae were recorded as having made a choice when they touched the distal end of one of the upper arms of a Y-tube. Larvae that remained motionless for 3 
min were recorded as non-responsive. Following the methods of Natale et al. (2004), larvae that moved but did not approach the ends of the arms within 5 min were recorded as non-choosers. After each trial the Y-tube and was thoroughly cleaned and dried. The position of red and green leaves within the left and right arms of the Ytubes was alternated to control for directional biases.

After the trials the larvae were placed in individual test tubes containing a matched pair of red and green $P$. colorata leaves for $48 \mathrm{~h}$. These caterpillars were re-tested according to the same protocol above. Sample sizes of naïve and experienced trials were different due to two deaths and two larvae entering pupal stage.

\subsubsection{Statistics}

Leaves, especially damaged leaves, produce complex blends of odours. SPME combined with GC-MS allows qualitative and semi-quantitative analysis of many volatile compounds from each sample. For each chromatogram, I used the integrated area under each peak to calculate the relative contribution ( $\%$ area) of that compound to the total volatile analyte. However, because some of the compounds share common precursors, it was not appropriate to treat each peak as statistically independent (Hare 2011). Therefore, I subjected the area \% values to principal components analysis (PCA) with varimax rotation. PCA is a widely used statistical tool for analysing VOC profiles (Pareja et al. 2009, Hare 2011). PCA takes a large number of possibly correlated variables and calculates a smaller number of independent and uncorrelated variables (principal components) that still retain the general patterns of variation of the original data. I used MANOVA upon the principal component scores to look for differences in VOC profiles between red or green matched pairs, using 'Pair' and 'Colour' as fixed factors. For the herbivore-induced experiment I included another fixed factor ('Eaten') in the MANOVA model, to distinguish leaves that had incurred herbivory from those that hadn't. For MANOVA analyses where a low sample size constrained the maximum number of explanatory variables, I reduced the number of principal components in the model, excluding those that explained the least variation first. After each MANOVA I used discriminant function (DF) analysis to find the linear combination of principal components that best separates the groups. The classification accuracy of the discriminant function was assessed using a cross- 
validation procedure, which removed each individual iteratively and attempted to predict its group identity.

To test for differences in the levels of sesquiterpenes in the VOC profiles of red and green mechanically damaged leaves, I used paired $t$-tests (after a Shapiro-Wilk test for normality) with a Bonferroni correction. Olfactory preferences of 'naïve' and 'experienced' $C$. obliquana larvae were analysed using a two-tailed exact binomial test. Larvae that did not make a choice were excluded from the analysis. PCA, discriminant function, binomial and $t$ - analyses were carried out using PSAW/SPSS Version 18.0 (Chicago, IL, USA). MANOVA and Shapiro-Wilk tests were carried out using $\mathrm{R}$ version 3.0.1 (R Core Team 2013).

\subsection{RESULTS}

\subsubsection{Passive night time VOC emissions}

There was considerable qualitative variability among the night time VOC emission profiles of red and green P. colorata trees (Fig. 3.4). Two of the trees, (one red and one green, from different pairs) emitted $54 \%$ more compounds, detected at greater than $2.5 \%$ of total ion count, than all the other trees. Twelve compounds were only detected in the profile of just one or the other of these two trees (indicated by a ++ symbol in Fig. 3.4). Only six compounds were present at detectable levels in all of the samples: unknown compound \#11, para-cymene, limonene, \#21, $\alpha$-copaene and $\alpha$-gurjunene. There were no significant differences in the VOC profiles of neighbouring matched red and green $P$. colorata trees (MANOVA \& DF, Table $3.1 \&$ Table 3.2) 


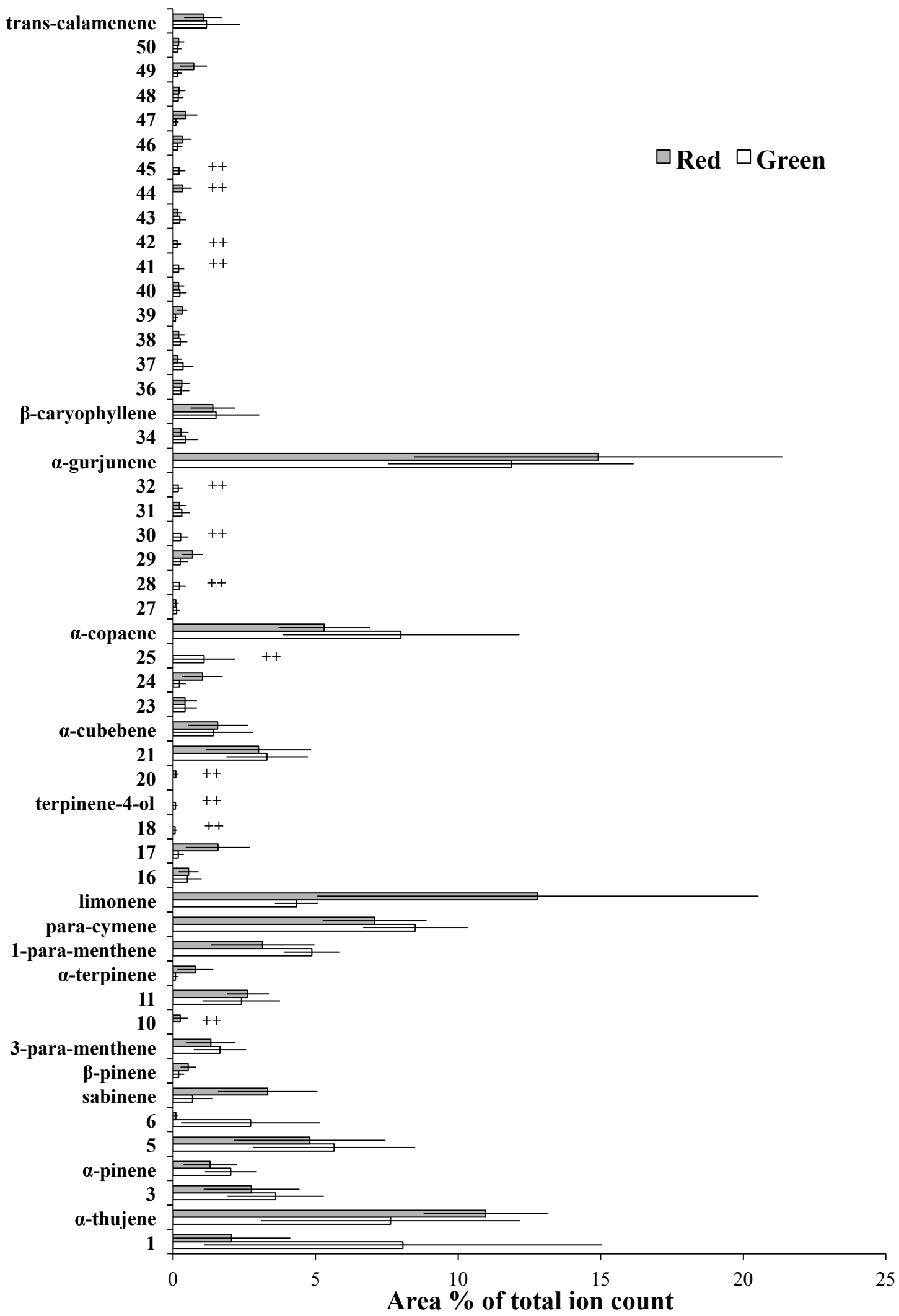

Fig. 3.4 Night time VOC emission profiles from leaves of red (shaded) and green (open) $P$. colorata trees in the field (Mean \pm SE, $\mathrm{n}=3$ pairs). There were no statistical differences between red and green matched pairs of trees (MANOVA and DF of principal components). Compounds that were only detected from a single tree are indicated by ++ . Numbers indicate unidentified compounds. 
Table 3.1 Results from MANOVA analyses of the relationships between the colour and VOC emission profiles (after dimension reduction by PCA) of intact, herbivore-, and mechanically-damaged leaves from matched pairs of P. colorata. Models included principal components as explanatory variables, and fixed factors shown below. Asterisk indicates statistical significance $(P<0.05)$.

\begin{tabular}{|c|c|c|c|c|c|}
\hline Experiment, Model and Effects & $V$ & d.f.NUM & d.f.DENOM & $F$-value & $P$ \\
\hline \multicolumn{6}{|l|}{ (a) Field leaves } \\
\hline \multicolumn{6}{|c|}{ PC1 + PC2 (together explaining $63 \%$ of variance) $\sim$ Colour + Pair } \\
\hline Colour & 0.62 & 2 & 1 & 0.82 & 0.62 \\
\hline Pair & 0.98 & 4 & 4 & 0.97 & 0.51 \\
\hline \multicolumn{6}{|l|}{ (b) Herbivore-damaged leaves } \\
\hline \multicolumn{6}{|c|}{$\mathrm{PC} 1+\mathrm{PC} 2+\mathrm{PC} 3+\mathrm{PC} 4$ (together explaining $88 \%$ of variance) $\sim$ Eaten + Colour + Pair } \\
\hline Eaten & 1.00 & 4 & 1 & 3800 & $0.0121 *$ \\
\hline Colour & 0.65 & 4 & 1 & 0.64 & 0.79 \\
\hline Pair & 2.15 & 12 & 9 & 2.15 & 0.17 \\
\hline \multicolumn{6}{|l|}{ (c) Mechanically-damaged leaves } \\
\hline \multicolumn{6}{|c|}{$\mathrm{PC} 1+\mathrm{PC} 2+\mathrm{PC} 3+\mathrm{PC} 4+\mathrm{PC} 5$ (together explaining $90 \%$ of variance) $\sim$ Colour + Pair } \\
\hline Colour & 0.63 & 5 & 5 & 1.73 & 0.28 \\
\hline Pair & 2.65 & 45 & 45 & 1.12 & 0.35 \\
\hline
\end{tabular}


Table 3.2 Results from Discriminant Function (DF) analyses of the relationships between the colour and VOC emission profiles (after dimension reduction by PCA) of intact, herbivore-, and mechanically-damaged leaves from matched pairs of $P$. colorata. Asterisk indicates statistical significance $(P<0.05)$.

\begin{tabular}{ccccc}
\hline Effect & $\Lambda$ & $\chi^{2}$ (d.f., n) & $P$ & $\begin{array}{c}\text { Squared canonical } \\
\text { correlation }\end{array}$ \\
& & \\
\hline
\end{tabular}

(a) Field leaves
Colour
0.63
$1.39_{(2, \mathrm{n}=6)}$
0.867
0.37

(b) Herbivore damaged leaves

$\begin{array}{lcccc}\text { Herbivory } & 0.07 & 15.83(4, \mathrm{n}=10) & \mathbf{0 . 0 0 3 *} & 0.93 \\ \text { Colour } & 0.81 & 1.27(4, \mathrm{n}=6) & 0.867 & 0.19\end{array}$

(c) Mechanically damaged leaves

\begin{tabular}{lllll} 
Colour & 0.58 & $8.37(5, \mathrm{n}=20)$ & 0.137 & 0.42 \\
\hline
\end{tabular}

\subsubsection{Herbivore-induced VOC emission}

Of the five pairs of excised leaves placed in vials with C. obliquana larvae, only two pairs of leaves incurred herbivory. VOC profiles emitted from P. colorata leaves that had been browsed differed both qualitatively and quantitatively to those that did not experience caterpillar herbivory. Three compounds were unique to either browsed or unbrowsed leaves: $\alpha$-pinene was only present in the VOC profiles of unbrowsed leaves; and two compounds were only detected from browsed leaves ( $\beta$-caryophyllene and 3-carene, indicated by a ++ symbol in Fig. 3.5). However, perhaps due to the low sample size, there was pronounced variability in VOCs between plants within treatments. $\beta$-Caryophyllene was only detected from leaves of one of the two plants that incurred herbivory. Similarly, $\alpha$-pinene was detected from leaves of only two of the three plants in the unbrowsed group. 


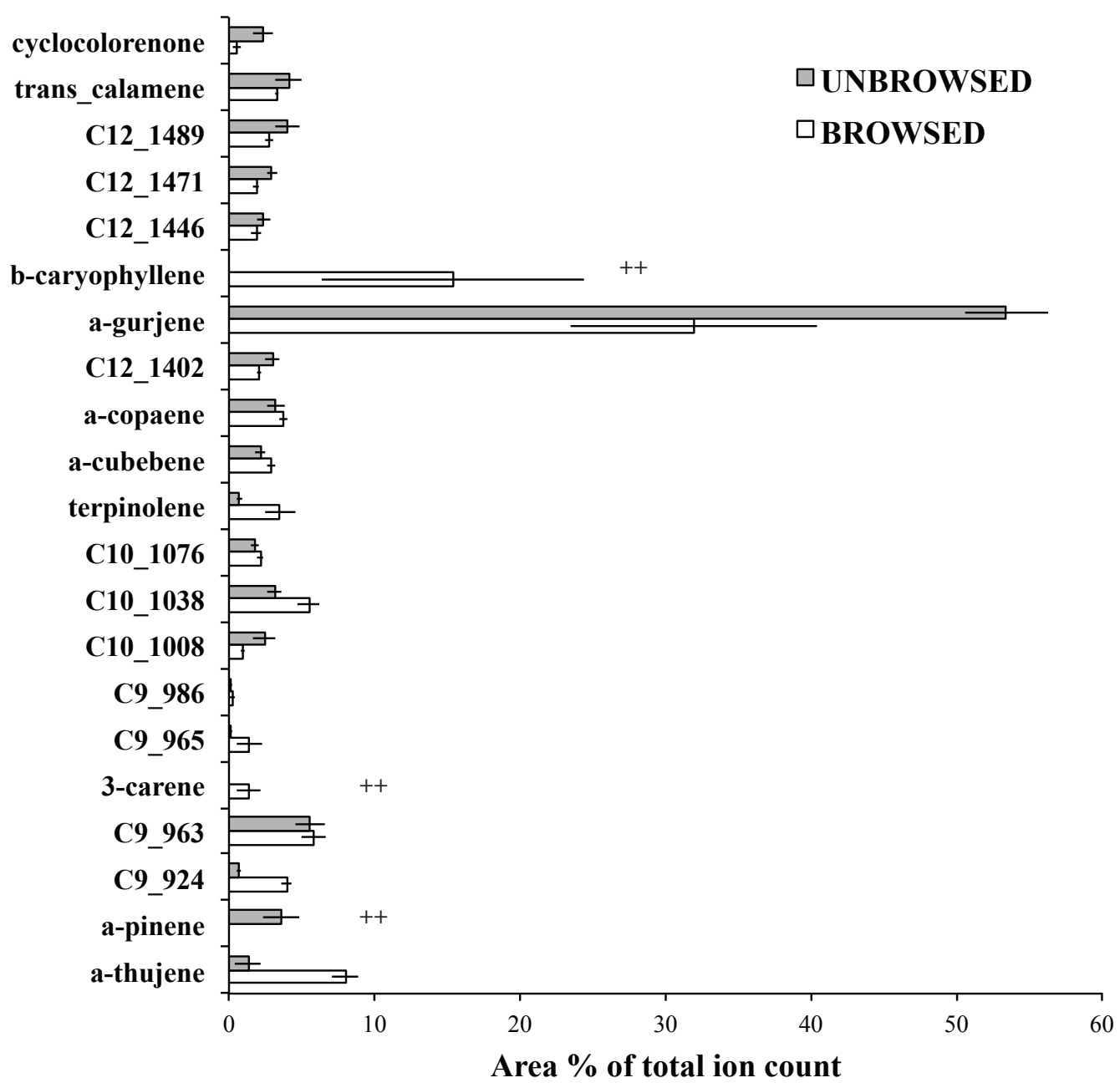

Fig. 3.5 VOC profiles of browsed ( $\mathrm{n}=2$ pairs) and unbrowsed $(\mathrm{n}=3$ pairs $) P$. colorata leaves (Mean $\pm \mathrm{SE}$ ). The VOC profiles from browsed and unbrowsed leaves were statistically distinguishable (MANOVA and DF of principal components). The symbol “++" denotes compounds unique either to browsed or unbrowsed leaves. RI_xxx indicates unknown compounds. 
MANOVA and DF analyses revealed that significant differences existed between the VOC profiles released from browsed and unbrowsed $P$. colorata leaves (Table 3.1 and Table 3.2). A very large amount of the variance in the data was accounted for by the discriminant function (squared canonical correlation $=0.93$, Table 3.2). The discriminant function correctly classified $100 \%$ of the samples as either browsed or unbrowsed leaves, however this accuracy dropped to $80 \%$ after cross-validation. The two components that most loaded upon the function that distinguished between browsed and unbrowsed leaves were: PC2 and PC3 (Table 3.3). However, a biplot of the scores from these two components did not reveal a clear pattern of separation (Fig. 3.6). MANOVA and DF analyses showed no consistent difference in volatiles profiles emitted between matched red and green leaves in either eaten or uneaten leaves (Table 3.1 and Table 3.2).

Table 3.3 Discriminant function (DF) structure matrix displaying unstandardized DF coefficients for each principle component calculated from the VOC profiles of browsed and unbrowsed $P$. colorata leaves.

\begin{tabular}{lc}
\hline Component & DF coefficient \\
\hline PC1 & -0.13 \\
PC4 & -0.01 \\
PC2 & 0.19 \\
PC3 & 0.24 \\
\hline
\end{tabular}




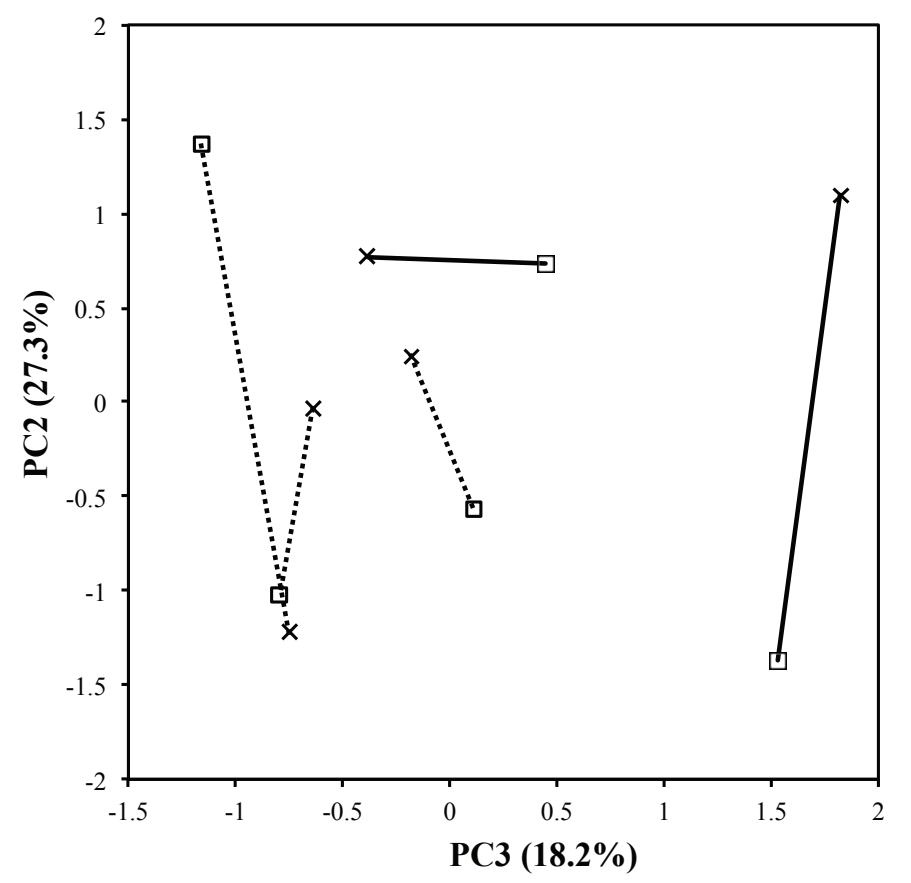

Fig. 3.6 Principal component biplot showing variation in VOC profiles for red ( $\square$ ) and green $(\mathrm{X})$ P. colorata leaves from potted plants exposed to herbivores. Lines join matched pairs of $P$. colorata leaves/trees. Dashed lines join matched pairs that did not experience herbivory.

\subsubsection{Mechanically damaged leaves}

Qualitatively, the VOC profiles of mechanically damaged leaves from P. colorata trees were less variable than those from the field and herbivore-induced VOC experiments (Fig. 3.7). Of the 20 samples studied, 14 emitted the same 25 compounds above detectable levels. Four of the six remaining samples were missing only one compound. The remaining two samples differed from the rest by emitting only 20 of 25 compounds. Mechanically damaged leaves from red and green $P$. colorata trees displayed no quantitative differences in VOC emission profiles (Table 3.1 and Table 3.2). I identified five different sesquiterpenes in the VOC profiles mechanically damaged leaves (cubebene, copaene, cadina-1,4-diene, calamanene and gurjenene), however paired $t$-tests revealed that red and green matched leaves did not differ in their levels of any of these five sesquiterpenes (five tests, all non-sig. even before Bonferroni correction, $\mathrm{n}=10$ pairs, d.f. $=9, t<1.4, P>0.2$ ). 


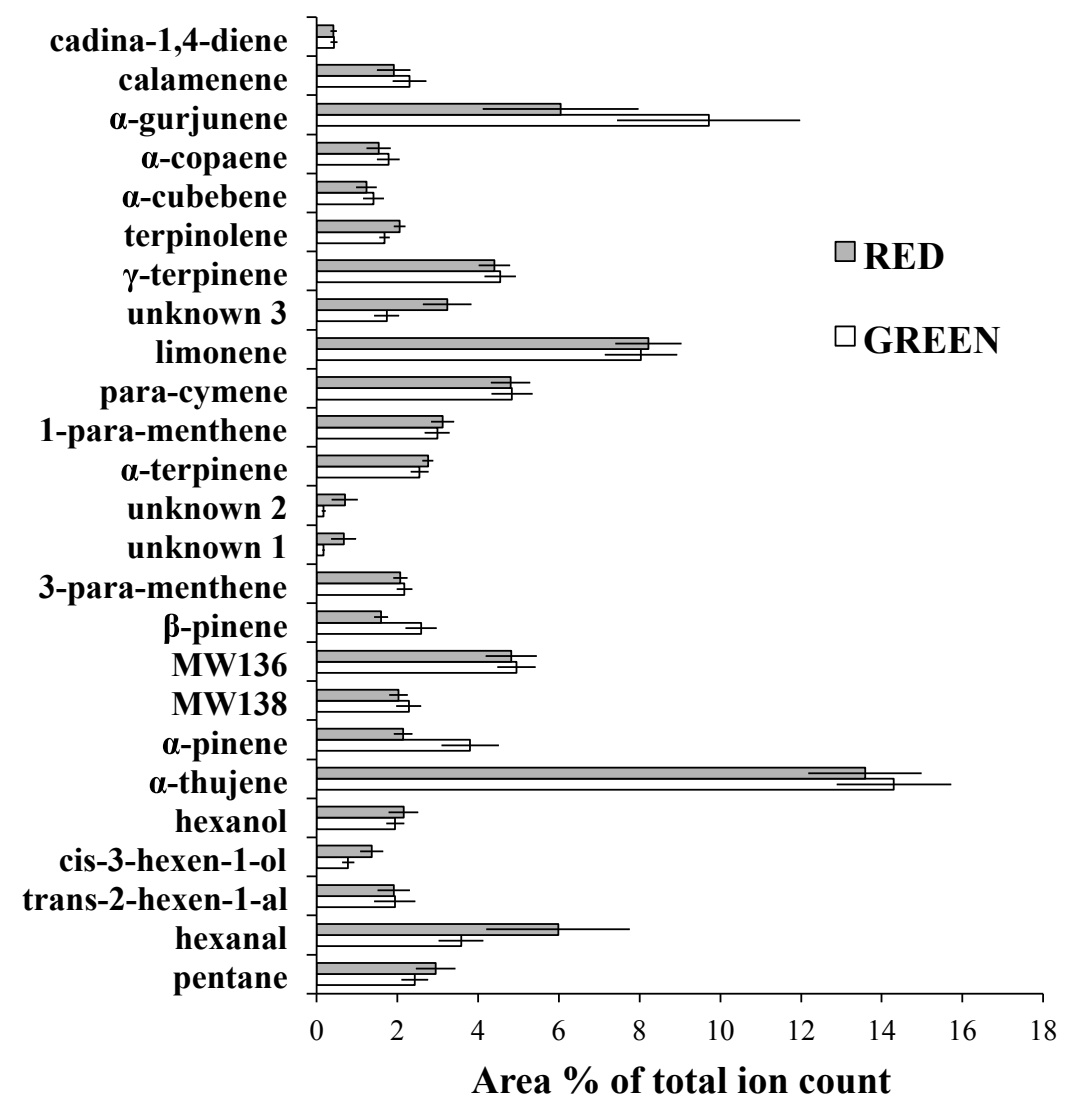

Fig. 3.7 VOC profiles from mechanically damaged leaves from red (shaded) and green (open) matched pairs of $P$. colorata trees (mean $\pm \mathrm{SE}, \mathrm{n}=10$ pairs). There were no statistical differences between red and green matched pairs of trees (MANOVA and DF of principal components). MWxxx indicates unknown compounds.

\subsubsection{Herbivore bioassays}

Lab-reared native C. obliquana caterpillars displayed no preference for VOCs released from mechanically damaged red or green $P$. colorata leaves (two-tailed exact binomial test, $\mathrm{n}=23, P=0.41$; Fig 3.8). After $48 \mathrm{~h}$ experience eating red and green $P$. colorata leaves, larvae still displayed no preference (two-tailed exact binomial test, $\mathrm{n}=19, P=1.0$; Fig. 3.8) 


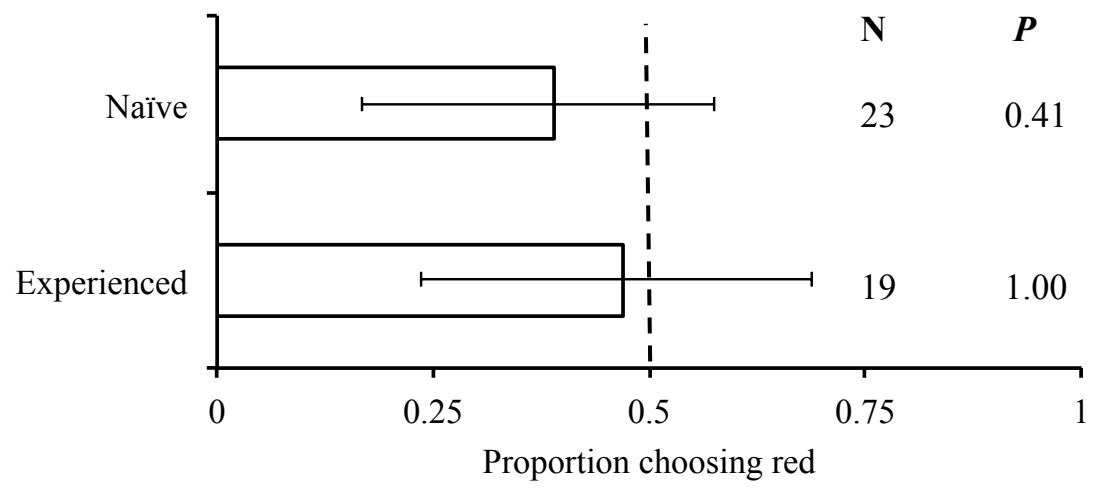

Fig. 3.8 Olfactory preferences of 'naïve' and 'experienced' $5^{\text {th }}$ instar C. obliquana larvae for mechanically damaged red- and green-coloured P. colorata leaves. Error bars (95\% C.I. with correction for continuity) that cross the dotted line indicate no significant preference for red or green leaves (two-tailed exact binomial test). Larvae that did not make a choice were excluded from the analysis.

\subsection{DISCUSSION}

I investigated the volatile profiles of differently coloured $P$. colorata leaves in relation to the signalling hypothesis that states that foliar anthocyanins may function as visual signals to deter approaching insect herbivores. None of my results indicate that olfactory cues from $P$. colorata leaves coincide with visual cues to signal defensive commitment against herbivory attacks. While the VOC profiles of browsed and unbrowsed leaves were statistically distinguishable, the VOC profiles released from intact, herbivore-, and mechanically-damaged $P$. colorata leaves did not reliably identify leaf colour (Table 3.1 and Table 3.2). Mechanically damaged red P. colorata leaves did not release statistically significant higher levels of sesquiterpenes than damaged green leaves. I did not identify any compounds known to be strong deterrents, such as methyl salicylate or farnesene. Moreover, naïve and experienced C. obliquana larvae displayed no preference for the volatiles from mechanically damaged red or green leaves (Fig. 3.8). Therefore, I conclude that VOC compounds are not likely to play a large role in mediating insect herbivore-plant interactions in $P$. colorata. 
It can be difficult to assess whether a non-significant result reflects reality or a Type II error. The statistical techniques I used were able to distinguish differences in VOC profiles. MANOVA and DF analyses statistically differentiated the VOC blends emitted from browsed and unbrowsed leaves (Table 3.1 and Table 3.2); This demonstrates that that my analyses had sufficient power to detect a difference even at a fairly low sample size ( $\mathrm{n}=10$ for herbivore-induced volatiles). However, no difference was detected among the VOC profiles of mechanically damaged red and green matched pairs of $P$. colorata leaves $(\mathrm{n}=20)$. A larger sample size gives greater statistical power to detect differences, therefore the variation in VOC profiles among mechanically damaged red and green $P$. colorata must be much smaller than the variation among VOC profiles of browsed and unbrowsed leaves. When considered along with the lack of olfactory preferences displayed by $C$. obliquana larvae, this strengthens my assumption that the lack of detectable difference in VOC profiles of red and green leaves is indeed a valid result rather than Type II error. Moreover, redder $P$. colorata leaves contained higher levels of a potent antifeedant called polygodial (Chapter 2), however there was no difference in the levels of volatile sesquiterpenes emitted from red and green mechanically damaged $P$. colorata leaves. Therefore, VOCs released from damaged $P$. colorata leaves are not a reliable cue of the defensive status of those leaves. These data do not support the hypothesis that leaf colour may correlate with leaf VOC emissions for olfactory signalling (Archetti and Brown 2004, Ougham et al. 2005b, Holopainen 2008, Döring et al. 2009, Lev-Yadun and Gould 2009).

In the introduction of this chapter I hypothesised that a plant might be expected to utilise both visual- and olfactory-based signalling, restricting the release of VOCs to times when nocturnal herbivores are active. I did not detect any consistent differences between the nocturnal VOC profiles released from red and green $P$. colorata trees in the field (Table 3.1 and Table 3.2). The VOC profiles were predominantly made up of monoterpenes and some sesquiterpenes. The relatively low sample sizes preclude unequivocal conclusions as to whether or not VOC compounds released from $P$. colorata in the field function for pre-damage olfactory signalling. However, given the lack of statistically distinguishable differences in volatile profiles between damaged red and green $P$. colorata leaves, it seems like an improbable scenario. 
My results differ from those of Holopainen et al. (2010) who did find differences in the VOC profiles of green and yellow Betula pendula leaves. However, yellow leaves of $B$. pendula are senescing and therefore there are many concomitant differences between green and yellow leaves, aside from colour. For example, in senescing leaves photosynthetic machinery is broken down and reabsorbed in preparation for leaf abscission (Holopainen et al. 2010). In contrast, differences in colouration of $P$. colorata leaves correspond to differences in the relative amounts of chemical defences and pigments, but not large changes in leaf function or physiology.

Red P. colorata leaves contained higher levels of polygodial (see Chapter 2). While polygodial is not known to be volatile, I hypothesised that other, more volatile, sesquiterpenes may be present in red leaves. Mechanical damage did not lead to the release of greater amounts of sesquiterpenes from red than green leaves (Fig. 3.7). However, epifluorescence microscopy indicates that the cell walls of idioblasts are highly lignified (K. S. Gould pers. comm.), and tearing the leaves, as was conducted in my experiment, might not necessarily have ruptured the idioblast cell walls (J. W. van Klink pers. comm.).

I hypothesised that if there were differences in VOC profiles between mechanically damaged red or green leaves, then this would affect the behaviour of leafroller larvae, a highly abundant pest upon $P$. colorata. Lab reared $C$. obliquana did not display innate behavioural preference for the VOC profiles released from mechanically damaged red or green $P$. colorata leaves. After feeding upon red and green $P$. colorata leaves for $48 \mathrm{~h}$ they did not display any learned preference (Fig. 3.8). Larval Ctenopseustis spp. leafrollers do exhibit innate gustatory preference for green over red leaves (see Chapter 5). Visual cues are important in mediating larval C. obliquana feeding decisions (Cooney et al. 2012). My results indicate that olfactory cues from mechanically damaged leaves are not, in isolation from other cues, associated with leaf-colour specific behaviour in C. obliquana larvae. Therefore, differences in the numbers of leafroller caterpillars between differently coloured $P$. colorata trees in the field (see Chapter 2) are unlikely to be a result of larval olfactory preferences.

One mechanism by which a leaf-colour dependant distribution of leafroller larvae in the field may occur (see Chapter 2) is if gravid female moths oviposit more frequently 
on green than on red P. colorata trees. It is known that other tortricid moths have olfactory receptors that are sensitive to GLVs, mono- and sesquiterpenes (Suckling et al. 1996, Jordan et al. 2009) and that GLVs and terpenes from host plants stimulate oviposition (Grant et al. 2007). However, the specificity of oviposition preferences of gravid lepidopteran females ranges from completely random oviposition (Fred and Brommer 2010), to requiring specific co-occuring stimulant and deterrent VOC host cues (Honda 1995, Bruce et al. 2005). Therefore, it is difficult to predict a-priori the oviposition preferences of Ctenopseustis spp. leafroller moths for red vs. green $P$. colorata leaves. My attempts at assaying the oviposition preferences of C. obliquana moths were unsuccessful (see Chapter 5). However, the VOC profiles of intact, herbivore-, or mechanically-damaged red and green $P$. colorata leaves did not statistically differ. Therefore, evidence does not support the hypothesis that female $C$. obliquana moths use VOC cues to inform of the defensive status of their potential host plants.

The VOC profiles emitted from browsed leaves could be statistically distinguished from those of un-browsed leaves (Table 3.1 and Table 3.2). In Chapter 2, I reported that herbivore damage correlated with leaf colour. Potentially, differences in VOC profiles, caused by colour-correlated differences in damage, may serve as a cue to inform gravid females of the relative herbivory levels of different $P$. colorata individuals. However, for this to explain the patterns of C. obliquana larvae distribution observed in Chapter 2 (i.e. more larvae upon green individuals), Ctenopseustis females would have to prefer to lay eggs upon previously damaged hosts. Usually, mated female Lepidoptera prefer to oviposit on undamaged plants (Carroll et al. 2006). Despite how speculative this mechanism is, as an alternative explanation for patterns consistent with a signalling function for leaf colour, it deserves further investigation.

Differences in numbers of caterpillars between red and green trees may also be caused by increased predation or parasitism of caterpillars upon red trees. (Whitman and Eller 1992, Xu et al. 2006). VOCs are used as cues by insect predators and parasitoids of lepidopteran larvae (Kessler and Baldwin 2001), even by insectivorous birds (Mäntylä et al. 2008), to locate their prey. Holopainen et al. (2010) found that senescing yellow leaves emitted less GLV as compared to green leaves, and 
concluded that senescing leaves were less able to defend themselves via these tritrophic interactions. However, I did not find significant differences in VOC profiles between red and green $P$. colorata. My results suggest that VOC-mediated tritrophic interactions are not a significant force structuring herbivore populations upon differently coloured $P$. colorata individuals.

Leaf VOCs might be expected to co-vary with leaf colour for reasons other than plant defence. Production of anthocyanin pigments and volatile terpenoid compounds are both common abiotic stress responses (Gould 2004, Vickers et al. 2009). Independently, anthocyanins and certain VOCs have been shown to mitigate the effects of oxidative stress (Gould et al. 2002a, Gould 2004, Vickers et al. 2009). However, as far I am aware, no study has simultaneously investigated VOC emissions and leaf pigments in relation to oxidative stress. I did not detect any isoprene (the most well-studied volatile in terms of abiotic stress; Vickers et al. 2009). I did detect monoterpenes and sesquiterpenes in the VOC profiles from $P$. colorata in the field; some monoterpenes and volatile sesquiterpenes are known to neutralise reactive oxygen species (Calogirou et al. 1999). These VOCs did not correlate with the leaf pigment differences between the red and green $P$. colorata trees. The field VOCs were collected when irradiance levels were extremely low, and perhaps the patterns of VOC release would differ between red and green when under more stressful conditions. However, Chapter 4 of this thesis details my investigations into the photosynthesis of red and green $P$. colorata trees, and I did not find colour-correlated differences in photoinhibition under high light conditions.

There is increasing evidence that HIPVs, and particularly herbivore-induced GLVs, can cause undamaged leaves to up-regulate their defence pathways and increase VOC emission; this priming can even occur between neighbouring plants (Ruther and Kleier 2005, Dudareva et al. 2006, Himanen et al. 2010). While the role of VOCs in plant-plant interactions in P. colorata have not been studied, if this priming were to occur between trees of different colour it would add noise to a co-evolved visual and/or olfactory signalling relationship.

Sample size was constrained by the large effort required to collect night time VOC profiles in the field and by the availability of SPME fibres and holders. I used two 
approaches to try to analyse differences in the complete VOC profiles between red and green $P$. colorata leaves: PCA and MANOVA+Discriminant function analyses. For both techniques most authors recommend much larger sample sizes than I was able to collect (Meyers et al. 2006). While there was good concordance among the tests and the findings of other chapters in this study, future experiments should focus on maximising sample size.

\subsection{CONCLUSIONS}

Red and green $P$. colorata trees in the field emit mostly volatile monoterpenes and some sesquiterpenes at night time. However, after herbivore and mechanical damage, the VOC profiles released from $P$. colorata leaves contained many more compounds above detectable levels, including a suite of GLVs. While the VOC profiles of browsed and unbrowsed leaves were statistically distinguishable, the VOC profiles released from intact, herbivore-, and mechanically-damaged $P$. colorata leaves did not reliably identify leaf colour. Moreover, naïve and experienced C. obliquana larvae displayed no preference for the volatiles from mechanically damaged red or green leaves. These results strengthen the assumption of Chapter 2 that differences in the numbers of leafroller larvae between red and green matched $P$. colorata individuals is the result of visual signalling rather than alternative VOC-mediated mechanisms. 


\section{Photosynthesis of red and green $P$. colorata leaves}

\subsection{ABSTRACT}

It has been hypothesised that foliar anthocyanin pigments function as visual signals to deter insect herbivores. However, studies of leaf signalling rarely consider the influence of these light-absorbing pigments upon photosynthesis. I compared the photosynthetic and photoinhibitory responses of red and green leaves from matched, neighbouring pairs of $P$. colorata of contrasting colour. Due to shading caused by anthocyanin pigments, I hypothesised that redder leaves would have a lower maximum photosynthetic assimilation rate and, after stress with white light, show a smaller reduction in photosynthetic efficiency than matched green leaves. Redder $P$. colorata leaves in the field had a lower $A_{\text {sat }}$ values than matched green leaves from neighbouring trees. However, I was unable to detect any measurable advantage in terms of photoprotection in the red P. colorata leaves as indicated by chlorophyll fluorescence profiles. My results indicate that the presence of anthocyanin pigments within mature, non-senescing leaves may impose a slight photosynthetic cost to the plant.

\subsection{INTRODUCTION}

Non-green leaf colouration is widespread, and, among other hypotheses, has been postulated to function for visual communication between plants and insect herbivores (Archetti 2000, Hamilton and Brown 2001, Cooney et al. 2012). The leaf signalling hypothesis was originally developed for autumn senescing leaves (Archetti 2000, Hamilton and Brown 2001) for which photosynthesis is no longer the chief role, and when the degradation of chlorophyll and the resorption of leaf nutrients are extremely important (Hoch et al. 2001, Holopainen and Peltonen 2002, Hoch et al. 2003, Ougham et al. 2005a). Signalling has also been hypothesised as an explanation of the widespread phenomenon of anthocyanin accumulation in young flushing leaves (Karageorgou and Manetas 2006); this colouration is usually transient, present only during leaf expansion, before the full complement of chlorophyll and maximum photosynthetic capacity has developed (Dodd et al. 1998, Manetas et al. 2002, Choinski Jr et al. 2003, Hughes et al. 2007). However, the possibility of visual 
signalling may be less beneficial for mature, non-senescing leaves which function primarily as photosynthetic organs, providing the majority of the carbon and energy required for plant growth and reproduction. Modifying the colour of leaf tissues through the accumulation of red anthocyanin pigments can alter the photosynthetic performance of leaves (Steyn et al. 2002, Close and Beadle 2003, Gould 2004); the impact of this upon plants whose leaves contain pigments for the function of signalling is unknown.

Signalling by maintaining anthocyanin pigments in mature non-senescing leaves may compromise the life-time photosynthetic contribution of a leaf. Anthocyanin pigments in red leaves absorb green light that otherwise might be used for photosynthesis of lower cell layers (Neill and Gould 1999, Nishio 2000, Feild et al. 2001, Gould et al. 2002b, Hughes et al. 2005, Karageorgou and Manetas 2006, Hughes and Smith 2007a), but see (van den Berg et al. 2009) who found different results for juvenile and senescing Acer saccharum leaves. As a consequence of light attenuation, red leaves from exposed locations frequently exhibit the characteristics of shade-acclimated leaves: smaller chlorophyll $a: b$ ratio (i.e. biased towards maximising light capture rather than electron flow into photosystem II reaction centres) (Boardman 1977, Givnish 1988, Manetas et al. 2003, Hughes and Smith 2007b, Kyparissis et al. 2007, Zeliou et al. 2009, Nikiforou and Manetas 2010) and lower light-saturated photosynthetic rate, as measured by $\mathrm{CO}_{2}$ assimilation, than comparable green leaves of the same species (Gould et al. 2002b, Nielsen and Simonsen 2011, Nikiforou et al. 2011, Zhang et al. 2011). Therefore when herbivory pressure is low (i.e. when signalling does not result in less predation) the production of foliar anthocyanins may present a physiological disadvantage.

On the other hand, signalling via foliar anthocyanin pigments may augment the photosynthetic performance of a leaf by reducing the negative impacts photooxidative stress. Many plants regularly experience an oversupply of light (Long et al. 1994, Alves et al. 2002, Wilhelm and Selmar 2010). Additionally, cold temperatures can lead to a reduced ability to assimilate light energy (Hughes et al. 2005). Excess light and/or cold temperatures can result in the formation of supernumerary reactive oxygen intermediates and consequently, photo-oxidative damage (Demmig-Adams and Adams III 1992, Mittler 2002, Demmig-Adams and Adams III 2006). There is 
strong empirical evidence that foliar anthocyanins protect from photooxidative stress, both by absorbing free radicals (Gould et al. 2002a, Neill et al. 2002b, a, Neill and Gould 2003, Kytridis and Manetas 2006, Zhang et al. 2012), and by attenuating high energy quanta surplus to the requirements for photosynthesis. Following exposures to saturating light flux, red leaves tend to be less photoinhibited and/or recover more quickly than comparable green leaves (Feild et al. 2001, Manetas et al. 2003, Neill and Gould 2003, Hughes et al. 2005, Gould et al. 2010, Nielsen and Simonsen 2011). Therefore, in times of cold and/or high light stress, foliar anthocyanin pigments may provide an additional benefit to signalling plants.

Only one study to date has simultaneously considered the signalling, photosynthetic shading and photoprotection hypotheses for foliar anthocyanin pigments, though this used young, developing green and red leaves rather than mature leaves (Karageorgou and Manetas 2006). The authors found that red leaves of Quercus coccifera benefitted in terms of reduced herbivory, but that there was little difference in photosynthesis and photoprotection between red and green young leaves. However, for leaves that contain anthocyanins throughout their life, it is not known how these possible roles of signalling, photoabatement and photoprotection might interact. The impacts of such interactions on plant fitness, and the co-evolutionary dynamics of plant-insect signalling are as yet unstudied.

Pseudowintera colorata is a small tree for which the leaves of some individuals contain anthocyanin pigments throughout the majority of their lifetime. Earlier in this thesis I showed that red and green matched pairs of $P$. colorata had different patterns of colouration, defence and insect pressure consistent with leaf signalling, with red bushes experiencing reduced rates of herbivory and hosting fewer herbivorous caterpillars (see Chapter 2). Here, I present the findings of my investigation into the consequences to photosynthesis of maintaining anthocyanin pigments within mature, non-senescing $P$. colorata leaves. Because of the inherent property of coloured anthocyanins in cell vacuoles to absorb visible light, I hypothesised that redder leaves would have a lower photosynthetic rate than matched green leaves from neighbouring individuals. In addition, as my field site is situated on an exposed ridge that frequently experiences bright sunlight, strong winds and low temperatures, I investigated if anthocyanin pigments exerted any measurable photoprotective role within P. colorata 
leaves. I compared the photoinhibitory responses of red and green leaves to light stress from either red/blue or white light. As it is not absorbed by anthocyanin pigments (Karageorgou and Manetas 2006, Hughes and Smith 2007b), I predicted no difference in the photoinhibitory responses of red and green leaves to red/blue light. However after stress with white light, I hypothesised that red leaves would show a smaller reduction in photosynthetic efficiency than green leaves.

\subsection{MATERIALS AND METHODS}

\subsubsection{Plant material}

All leaves used in this experiment were taken from a subsample of the matched pairs of $P$. colorata trees at the Wellington site established in Chapter 2 of this thesis following the methods of Hagen et al. (2004). Each pair contained one tree whose leaves were predominantly green and one tree whose leaves were much redder. The 10 pairs that displayed the greatest within-pair contrast in colouration were preferentially selected. The individuals in each pair were located no more than $2 \mathrm{~m}$ apart from one another, and were closely matched in size, exposure to direct sunlight and prevailing winds. Within each tree, one stem was selected from the periphery of the north face of the canopy. Within pairs, the stems were closely matched for azimuth, exposure to direct sunlight and prevailing winds. Stems whose leaves showed signs of significant herbivore or mechanical damage were excluded. This design allowed me to investigate the effect of leaf colour on photosynthesis whilst minimising differences in environmental conditions.

\subsection{2 $\mathrm{CO}_{2}$ assimilation measurements}

With the assistance of Kaylyn Carpenter (High Point University, NC, USA), I collected photosynthesis data from matched pairs of $P$. colorata in the field. Sampling took place between 0930 and 1530, over three days during Autumn 2012. The mean daily temperature of these three days ranged from 13 to $17^{\circ} \mathrm{C}$ (Table 4.1; NIWA National Climate Database, Kelburn Weather Station, http://cliflo.niwa.co.nz; accessed 10 July 2012). The impact of variation in climate within and among sampling days upon photosynthesis was minimised, as both trees of each pair were measured consecutively on the same day. 
Table 4.1 Median and interquartile range $\left[\mathrm{IQR}=\mathrm{Q}_{1}\right.$ to $\left.\mathrm{Q}_{3}\right]$ for air temperature $\left(\mathrm{T}_{\text {air }}\right)$, relative humidity $(\mathrm{RH})$, and wind speed during sampling days.

\begin{tabular}{lccc}
\hline Climate data & 25 April 2012 & 26 April 2012 & 4 May 2012 \\
\hline $\mathrm{T}_{\text {air }}\left({ }^{\circ} \mathrm{C}\right)$ & $17.1(16.4-17.3)$ & $15.3(15.1-15.5)$ & $12.9(12-13.8)$ \\
$\mathrm{RH}(\%)$ & $63(59-66)$ & $84(82-89)$ & $78(75-83)$ \\
Wind speed $\left(\mathrm{m} \mathrm{s}^{-1}\right)$ & $5.0(4.8-5.4)$ & $3.9(3.4-4.1)$ & $3.1(2.9-3.6)$ \\
\hline
\end{tabular}

From each of the selected stems (still attached to the plant), one leaf was carefully chosen (from the third to fifth youngest fully expanded leaf). Leaves with significant herbivore or mechanical damage were excluded. Light response curves for $\mathrm{CO}_{2}$ assimilation were done using a LI-6400 photosynthesis system with red and blue LED light sources (LI-COR Biosciences, Lincoln, NE, USA). $\mathrm{CO}_{2}$ concentration was maintained at $400 \mu \mathrm{mol} \mathrm{CO} \mathrm{mol}^{-1}$ air. Flow rate of air through the leaf chamber was maintained at $500 \mu \mathrm{mol} \mathrm{s}^{-1}$. The light ramp began at $1500 \mu \mathrm{mol} \mathrm{m} \mathrm{m}^{-1}$ and decreased progressively until the irradiance was zero, with 1 min pauses between measurements. I used a 2 x $6 \mathrm{~cm}$ leaf chamber. As photosynthesis was recorded on a per-unit-leafarea basis, I corrected for the surface area of any leaves that were smaller than $6 \mathrm{~cm}^{2}$. P. colorata leaves are hypostomatic (Sampson 1980).

At the end of each field day, each stem bearing a focal leaf was excised several $\mathrm{cm}$ below the focal leaf at a $45^{\circ}$ angle. The cut stems were immediately recut under water and held in $5 \mathrm{~mL}$ florists' tubes containing fresh water throughout the subsequent experiments.

The leaves of one tree repeatedly detached from branches during several attempts at acquiring $\mathrm{CO}_{2}$ light curves. The $\mathrm{CO}_{2}$ assimilation data from this pair was not included in the analyses, however a similar stem from this plant was excised for chlorophyll fluorescence measurements and pigment analysis

From the light response curves for $\mathrm{CO}_{2}$ assimilation, I calculated: dark respiration rate (respiration at zero irradiance), light compensation point (light intensity where the rates of photosynthesis and respiration are equal), apparent maximum quantum yield/ photosynthetic efficiency (initial slope of the light curve), light saturation point (light 
intensity at which photosynthesis is saturated), and the saturated photosynthesis rate (light saturated $\mathrm{CO}_{2}$ assimilation rate, $\left.A_{\text {sat }}\right)$. These points were calculated using MS Excel (Microsoft Corporation, Redmond, WA, USA).

\subsubsection{Chlorophyll fluorescence and photoinhibition}

At the end of each sampling day, the excised stems were returned to the lab, darkadapted for $4 \mathrm{~h}$ and then the maximum quantum yield, estimated by the ratio of variable to maximum fluorescence $\left(F_{\mathrm{v}} / F_{\mathrm{m}}\right)$ was measured for the focal leaf from each stem using a PAM 2500 (Heinz Walz GmbH, Effeltrich, Germany) chlorophyll fluorometer. Following the $F_{\mathrm{v}} / F_{\mathrm{m}}$ measurements, each focal leaf was individually subjected to a rapid light curve, from $1 \mu \mathrm{mol}$ to $2000 \mu \mathrm{mol} \mathrm{m} \mathrm{m}^{-2} \mathrm{~s}^{-1} P A R$ (increasing in ten increments each lasting $25 \mathrm{~s}$ ) provided by red LEDs, with a maximum emission at $630 \mathrm{~nm}$. Estimates of photochemical quantum yield ( $\left.\Phi_{\text {PSII }}\right)$, photochemical quenching $(q P)$ and non-photochemical quenching $(N P Q)$ were recorded from the PAM software, following the formulae derived by Genty et al. $(1989,1990)$. Afterwards, these leaves were exposed to $0.5 \mathrm{~h}$ of $2000 \mu \mathrm{mol} \mathrm{m} \mathrm{m}^{-2}$ collimated white light from a Novaflex fibre optic illuminator (Fig. 4.1A). Light intensity was measured using LI250A light meter (Li-Cor Biosciences, Lincoln, NE, USA). Immediately after this photoinhibition treatment the effective quantum yield $\left(F_{\mathrm{v}}{ }^{\prime} / F_{\mathrm{m}}\right.$ ') of each leaf was measured with the PAM-2500.

\subsubsection{Digital image analysis}

After the $\mathrm{CO}_{2}$ assimilation and chlorophyll fluorescence measurements, the focal leaves were excised, scanned at 300 dpi using a CanoScan LiDE 20 desktop scanner (Canon, Tokyo, Japan) and sealed in plastic $20 \mathrm{~mL}$ vials in a $-80^{\circ} \mathrm{C}$ freezer until pigment extraction. Leaf area was measured from the digital images using ImageJ v1.41 (National Institutes of Health, Bethesda, MD, USA) following the methods of Cooney et al. (2012). Unfortunately the leaves from three of the pairs were not scanned and frozen until $48 \mathrm{~h}$ after being excised. Noticeable drying of these leaves occurred as well as changes in their colour. The data from these three pairs of leaves were excluded from the pigment extraction analysis. 


\subsubsection{Pigment analysis}

Pigment analyses were carried out by Luke Cooney (VUW, NZ) and Kaylyn Carpenter (High Point University, NC, USA). After retrieval from the $-80^{\circ} \mathrm{C}$ freezer, $P$. colorata leaves were thawed for $1 \mathrm{~min}$ so that they could be cut without shattering. $\mathrm{A} \sim 1 \mathrm{~cm}^{2}$ section of leaf lamina was excised from a point normal to the centre of the midrib ( $\sim 0.02 \mathrm{~g}$ fresh weight). After weighing, leaf material was placed in precooled $2.0 \mathrm{~mL}$ microcentrifuge tubes (Biotix, Inc., San Diego, CA, USA) containing $1.5 \mathrm{~mL}$ of $80 \%(\mathrm{v} / \mathrm{v})$ acetone and a $5 \mathrm{~mm}$ stainless steel ball bearing (Qiagen N.V., Venlo, Netherlands). The samples were agitated in a precooled bead mill (Tissuelyser LT, Qiagen N.V., Venlo, Netherlands) for 6 min at 50 oscillations s ${ }^{-1}$ and centrifuged at $13000 \mathrm{rpm}$ for $5 \mathrm{~min}$ in a microcentrifuge (5415 D, Eppendorf AG, Hamburg, Germany). Absorbance of the supernatant was measured at 470, 647 and $663 \mathrm{~nm}$ using a UV-2550 UV-vis spectrophotometer (Shimadzu Corp. Kyoto, Japan) and the concentrations of chlorophyll and carotenoid pigments calculated following the methods of Lichtenthaler (1987).

\subsubsection{Photoinhibition with different illumination}

To assess whether red pigments played a role in shading subjacent chloroplasts in $P$. colorata leaves, a further photoinhibition experiment was carried out. A further 2 stems, each bearing at least 5 fully expanded leaves were collected from each tree from the 10 matched pairs of $P$. colorata, using the selection and excision procedures as outlined above. A focal leaf was selected and marked as before.

The stems were placed in florist tubes, brought back to the lab, and dark-adapted overnight.

At beginning of following day, the $F_{\mathrm{v}} / F_{\mathrm{m}}$ value of each focal leaf was measured using the PAM-2500. Then the two sets of red and green matched focal leaves from each pair were subjected to $0.5 \mathrm{~h}$ of either $2000 \mu \mathrm{mol} \mathrm{m} \mathrm{m}^{-2} \mathrm{~s}^{-1}$ of collimated white light delivered by a Novaflex fibre optic illuminator fitted with a $115 \mathrm{v}$ Halogen bulb (Fig. 4.1A) or $2000 \mu \mathrm{mol} \mathrm{m} \mathrm{m}^{-2} \mathrm{red} / \mathrm{blue}$ led lights from the Li-Cor 6400-02B LED light source (Fig. 4.1B). Light intensity was measured using Li-Cor LI-250A. Immediately after the $0.5 \mathrm{~h}$ light treatment, I compared the $F_{\mathrm{v}}{ }^{\prime} / F_{\mathrm{m}}$ ' of red and green leaves using the PAM-2500. Due to equipment constraints, each pair was sequentially exposed to 
the photoinhibition and $F_{\mathrm{v}}{ }^{\prime} / F_{\mathrm{m}}$ ' processes throughout the day. Prior to undergoing the photoinhibition treatments, stems were kept in a dark cupboard at room temperature.
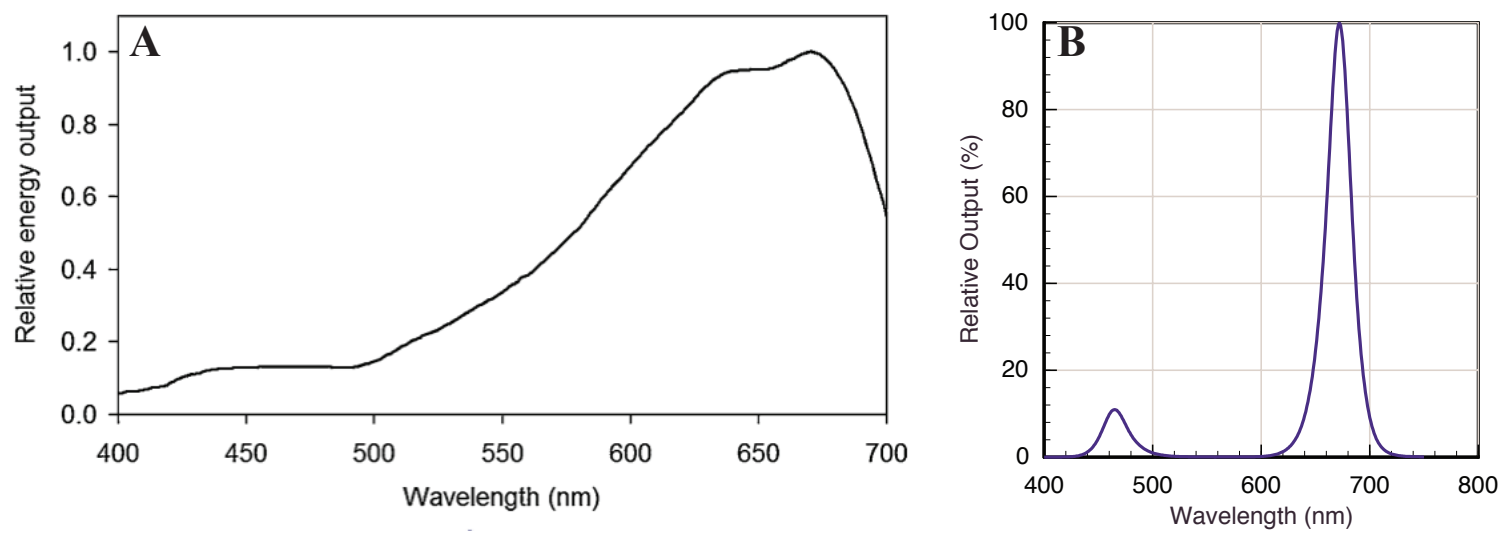

Fig. 4.1 Transmittance spectra from Novaflex fibre optic illuminator fitted with a 115v Halogen bulb (A, from Gould et al. 2010) and Li-Cor 6400-02B LED source (B, from figure 8-3 of Li-6400 Instruction Manual). B is deficient in green light.

\subsubsection{Statistical analyses}

The parameters of light response curves are frequently analysed using ANOVA, to test for differences among the group means of, say, photosynthesis of red and green trees at different light intensities, with the assumption that the data are independent. However, as trees in my study were not selected by random, my paired design violates this assumption of independence. Repeated measures ANOVA is classed as a 'within-subjects design' as it is traditionally used to analyse data from longitudinal studies where control and treatment groups represent the pre- and post-treatment responses from the same subject. Here, I treated each pair of matched red and green P. colorata trees as a subject, and used repeated measures ANOVA to test if the mean response differed among the different levels of light intensity (PAR, a factor with 10 or 15 levels depending on the light curve, see equation 1 below), and to assess whether the relationship between the response variable and light intensity differed within-subjects (i.e. within-pairs of neighbouring matched red and green P. colorata trees). 
I carried out multiple tests; one for each of the various parameters of the $\mathrm{CO}_{2}$ assimilation and chlorophyll fluorescence light response curves: $A, \Phi_{\mathrm{PSII}}, q P$, and $N P Q$. Normality within different light levels and tree colours was assessed using Shapiro-Wilk tests. The amount of variance explained by each factor was manually calculated from the sum-of squares (SS) of each test using equation 2 below (Levine and Hullett 2002).

$$
\eta^{2}=\mathrm{SS}_{\text {between }} / \mathrm{SS}_{\text {total }}
$$

Data from the photoinhibition experiments were also analysed using repeated measures ANOVA tests. Again, I treated pair as a subject, and tested for within-pair differences in the $F_{\mathrm{v}} / F_{\mathrm{m}}$ of both dark-adapted (factor "PREPOST" level 0 ) and photoinhibited (factor "PREPOST" level 1) matched red and green P. colorata (factor "LeafColour" with two levels, equation 3 below). Additionally, I tested for withinpair differences in $F_{\mathrm{v}} / F_{\mathrm{m}}$ after photoinhibition using two different types of illumination (white vs. red/blue, factor "LightColour" with two levels, equation 4 below).

$$
F_{\mathrm{v}} / F_{\mathrm{m}} \sim \text { PREPOST }+ \text { LeafColour }+ \text { PREPOST } * \text { LeafColour }
$$

PREPOST*LeafColour + PREPOST*LightColour + LeafColour*LightColour + PREPOST*LeafColour*LightColour

Differences in light curve parameters and pigment concentrations between red and green matched $P$. colorata leaves were assessed using either paired Student's $t$ - or Wilcoxon signed ranks test, depending on the outcome of a Shapiro-Wilk test. All statistical analyses were carried out using PSAW/SPSS Version 18.0 (Chicago, IL, USA). 


\subsection{RESULTS}

\subsubsection{Light response curves for $\mathrm{CO}_{2}$ assimilation}

Photosynthesis increased significantly with increasing light intensity $\left(F_{14,98}=48, P<\right.$ $\left.0.001, \eta^{2}=0.75\right)$. There were no statistical differences between matched red and green $P$. colorata leaves in any of the parameters for the response curves for $\mathrm{CO}_{2}$ assimilation $(A)$ at low light intensities: the dark respiration rate, light compensation point, apparent maximum quantum yield/photosynthetic efficiency and light saturation point of matched red and green $P$. colorata leaves did not differ significantly (Table 4.2).

Table 4.2 Parameters of the initial phase of $\mathrm{CO}_{2}$ assimilation light curves from matched green and red $P$. colorata leaves. Means of $\mathrm{n}=9 \pm$ SE. No statistical differences were found.

\begin{tabular}{lcc}
\hline Photosynthetic parameter & Green & Red \\
\hline Dark respiration rate $\left(\mu \mathrm{mol} \mathrm{CO}_{2} \mathrm{~m}^{-2} \mathrm{~s}^{-1}\right)$ & $-0.64 \pm 0.2$ & $-0.58 \pm 0.3$ \\
Light compensation point $\left(\mu \mathrm{mol} \mathrm{m}^{-2} \mathrm{~s}^{-1}\right)$ & $15 \pm 3.0$ & $18 \pm 5.0$ \\
Apparent max. quantum yield $\left(\Phi_{\mathrm{a}}\right)$ & $0.03 \pm 0.004$ & $0.02 \pm 0.003$ \\
Light saturation point $\left(\mu \mathrm{mol} \mathrm{m} \mathrm{s}^{-1}\right)$ & $622 \pm 70$ & $456 \pm 60$ \\
\hline
\end{tabular}

Overall, the relationship between photosynthesis and light intensity did differ significantly between differently coloured $P$. colorata trees $\left(F_{1,7}=5.7, P=0.049, \eta^{2}\right.$ $=0.0035$ ). Green $P$. colorata leaves (from greener trees) had significantly higher $\mathrm{CO}_{2}$ assimilation rates $(A)$ than red $P$. colorata leaves (from matched redder trees) at irradiances above $\sim 500 \mu \mathrm{mol} \mathrm{m}^{-2} \mathrm{~s}^{-1} \mathrm{red} / \mathrm{blue}$ light (Fig. 4.2). Leaves from green trees had $47 \%$ higher light saturated $\mathrm{CO}_{2}$ assimilation rate $\left(A_{\text {sat }}\right)$ than their matched red trees (paired Student's $t$-test, $\mathrm{n}=9$ pairs, $t=2.755, \mathrm{P}=0.025$, Fig. 4.3 ). In 8 of the 9 pairs, the redder leaves had the lower $A_{\text {sat }}$ (Fig. 4.3). Additionally there was a significant but extremely weak interaction between tree colour and light intensity $\left(F_{14,98}=2.4, P=0.007, \eta^{2}=0.0015\right)$. 


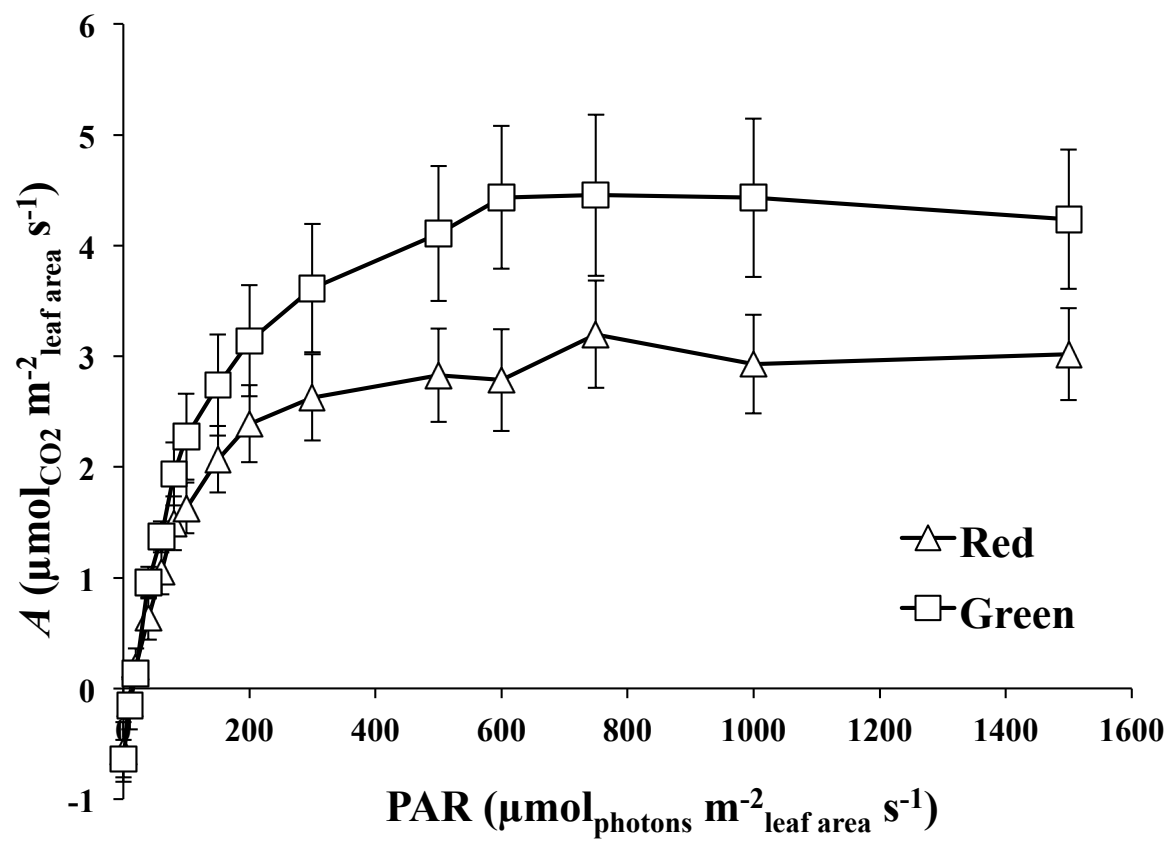

Fig. 4.2 Light response curves for $\mathrm{CO}_{2}$ assimilation $(A)$ of green $(\square)$ and red $(\Delta) P$. colorata leaves in the field. Means $\pm \mathrm{SE}, \mathrm{n}=9$. Green $P$. colorata leaves (from greener trees) had significantly higher $\mathrm{CO}_{2}$ assimilation rates $(A)$ than red $P$. colorata leaves (from matched redder trees, repeated measures ANOVA, $F_{1,7}=5.7, P=0.049$, $\left.\eta^{2}=0.0035\right)$ 


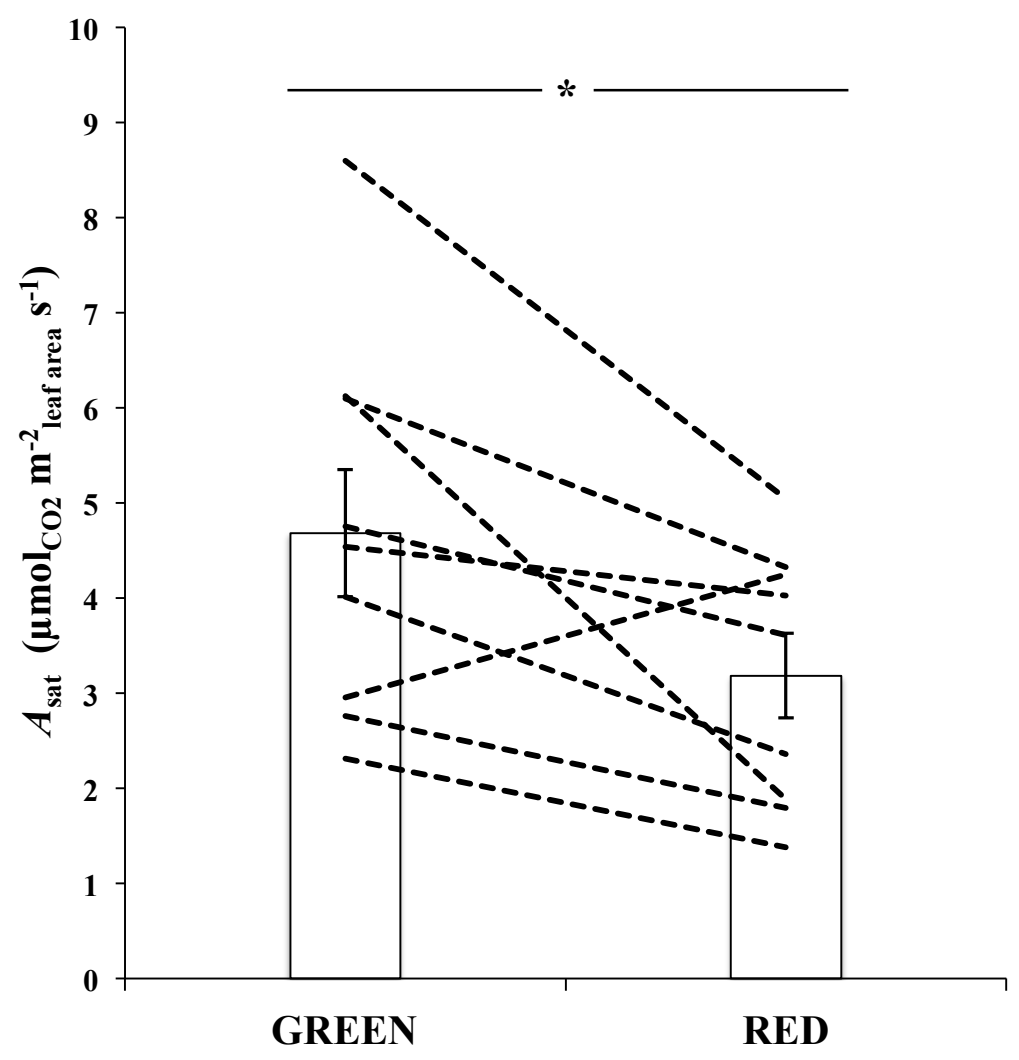

Fig. 4.3 Light saturated rate of $\mathrm{CO}_{2}$ assimilation $\left(A_{\text {sat }}\right)$ for matched green and red $P$. colorata leaves. Dashed lines connect the $A_{\text {sat }}$ values of matched leaves. Bars indicate group means \pm SE. Asterisk indicates statistical differences between matched leaves (paired Student's $t$-test, $\mathrm{n}=9$ pairs, $t=2.755, P=0.025$ ).

\subsubsection{Pigment analysis of red and green P. colorata leaves}

Several sequential natural $\log$ transformations were required for the chlorophyll $a$ data to conform to normality assumptions. It has been shown that when data are profoundly non-normal, the Wilcoxon signed-rank test can be appreciably more powerful than the Student's $t$ test (Blair and Higgins 1985). When applying a nonparametric Wilcoxon signed rank test, red $P$. colorata leaves contained significantly lower FW concentrations of chlorophyll $a(\mathrm{n}=7$ pairs, $\mathrm{Z}=-2.4, P=0.018)$ than did the green. Within all of the 7 pairs, the green leaf held a greater concentration of chlorophyll $a$ than the red individual though the magnitude of this difference varied considerably between $4 \mu \mathrm{g} \mathrm{g}^{-1} \mathrm{FW}$ and $369 \mu \mathrm{g} \mathrm{g}^{-1} \mathrm{FW}$ (Fig. 4.4). When the outlier pair was removed the difference in $\mathrm{Chl} a$ between green and red $P$. colorata leaves 
still approached statistical significance $(t=2.4, P=0.059, \mathrm{n}=6$ pairs $)$. Chlorophyll $b$ FW concentrations also tended to be lower in red $P$. colorata leaves (Wilcoxon signed rank, $\mathrm{n}=7$ pairs, $\mathrm{Z}=-1.9, P=0.063$ ) however this difference was not statistically significant. As a result there was no statistical difference in the ratios of chlorophylls $a$ to $b(\mathrm{Chl} a: b, \mathrm{n}=7$ pairs, $\mathrm{Z}=-0.5, P=0.61)$ between matched red and green $P$. colorata leaves.

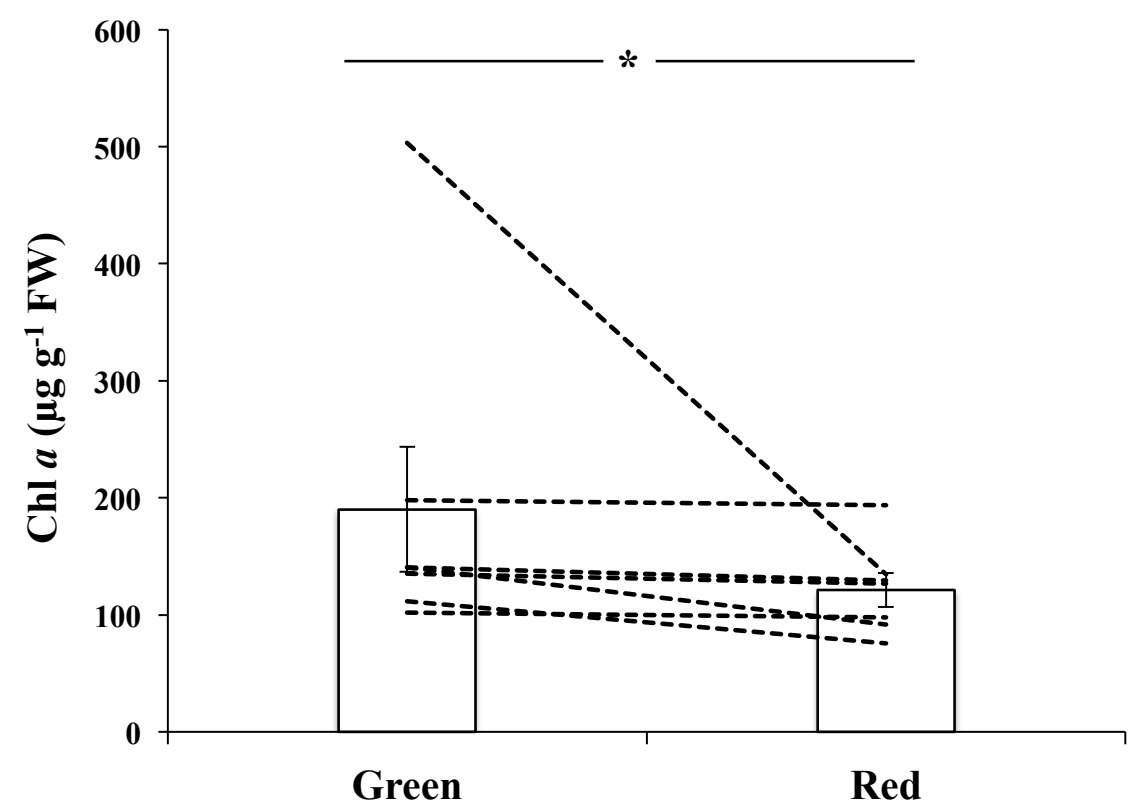

Fig. 4.4 Concentrations of chlorophyll (Chl) $a$ pigments in matched red and green leaves of $P$. colorata (Means $\pm \mathrm{SE}, \mathrm{n}=7$ pairs). Dashed lines join concentrations of matched leaves. Asterisk indicates statistical differences within pairs (Wilcoxon signed rank, $P<0.05)$

Green leaves contained significantly higher FW concentrations of total chlorophylls $\left(\mathrm{Chl}_{a+b}\right)$ than did red $(\mathrm{n}=7$ pairs, $\mathrm{Z}=-2.0, P=0.043)$. There were no statistical differences in $\mathrm{FW}$ concentrations of carotenoid pigments $\left(\mathrm{Car}_{\mathrm{x}+\mathrm{c}}, \mathrm{n}=7\right.$ pairs, $t=-0.6$, $P=0.56)$, nor in the ratios of carotenoids to total chorophylls between matched red and green leaves (Car: $\mathrm{Chl}_{a+b}, \mathrm{n}=7$ pairs, $t=-1.1, P=0.31$, Table 4.3). 
Table 4.3 Concentrations of chlorophylls (Chl) $a$ and $b$ and carotenoid $\left(\mathrm{Car}_{\mathrm{x}+\mathrm{c}}\right)$ pigments in matched pairs of red and green leaves of $P$. colorata (Mean $\pm \mathrm{SE}, \mathrm{n}=7$ pairs). Asterisk indicates statistical difference (Wilcoxon signed ranks test, $P<0.05$ )

\begin{tabular}{lcc}
\hline Pigment & Green & Red \\
\hline $\mathrm{Chl} a\left(\mu \mathrm{g} \mathrm{g}^{-1} \mathrm{FW}\right)$ & $190 \pm 53$ & $121 \pm 15 *$ \\
$\mathrm{Chl} b\left(\mu \mathrm{g} \mathrm{g}^{-1} \mathrm{FW}\right)$ & $203 \pm 65$ & $117 \pm 18$ \\
$\mathrm{Chl}_{a+b}\left(\mu \mathrm{g} \mathrm{g}^{-1} \mathrm{FW}\right)$ & $390 \pm 120$ & $240 \pm 32 *$ \\
$\mathrm{Chl} a: b$ & $1.0 \pm 0.08$ & $1.1 \pm 0.07$ \\
$\mathrm{Car}_{x+c}\left(\mu \mathrm{g} \mathrm{g}^{-1} \mathrm{FW}\right)$ & $10 \pm 4.0$ & $13 \pm 2.4$ \\
$\mathrm{Car}_{\mathrm{x}+\mathrm{c}}: \mathrm{Chl}_{a+b}$ & $0.04 \pm 0.02$ & $0.06 \pm 0.01$ \\
\hline
\end{tabular}

\subsubsection{Light response curves for chlorophyll fluorescence}

Quantum yield $\left(\Phi_{\mathrm{PSII}}\right)$ of both red and green $P$. colorata leaves decreased considerably with increasing irradiance $\left(F_{9,81}=730, P<0.001, \eta^{2}=0.97\right)$. While there was no statistically significant effect of tree colour on the overall relationship between $\Phi_{\mathrm{PSII}}$ and light intensity $\left(F_{1,9}=0.025, P=0.88\right)$, there was an extremely weak but significant interaction between the effects of tree colour and light intensity on $\Phi_{\text {PSII }}\left(F_{9,81}=4.6, P<0.001, \eta^{2}=0.004\right)$; green leaves exhibited a reduced decline in $\Phi_{\mathrm{PSII}}$ at higher irradiances (Fig. 4.5A).

The photochemical quenching coefficient $(q P)$ of red and green leaves also declined significantly with increasing light intensity $\left(F_{9,81}=510, P<0.001, \eta^{2}=0.97\right)$. However, for green leaves, the magnitude of the decline in $q P$ at higher irradiances was significantly less than for red leaves $\left(F_{1,9}=5.6, P=0.042, \eta^{2}=0.03\right)$. As both red and green $q P$ curves have the same origin, there was an extremely weak but significant interaction between tree colour and light intensity $\left(F_{9,81}=5.8, P<0.001\right.$, $\eta^{2}=0.002$, Fig. 4.5B).

Non-photochemical quenching $(N P Q)$ in all leaves increased significantly as light intensity increased $\left(F_{9,81}=270, P<0.001, \eta^{2}=0.89\right)$. Red $P$. colorata leaves had greater $N P Q$ than green at almost all irradiances $\left(F_{1,9}=7.0, P=0.026, \eta^{2}=0.02\right)$. As this difference in $N P Q$ between red and green leaves increased at higher irradiances, 
there was a significant interaction between tree colour and light intensity $\left(F_{9,81}=3.7\right.$, $P=0.001, \eta^{2}=0.008$, Fig. 4.5C). 

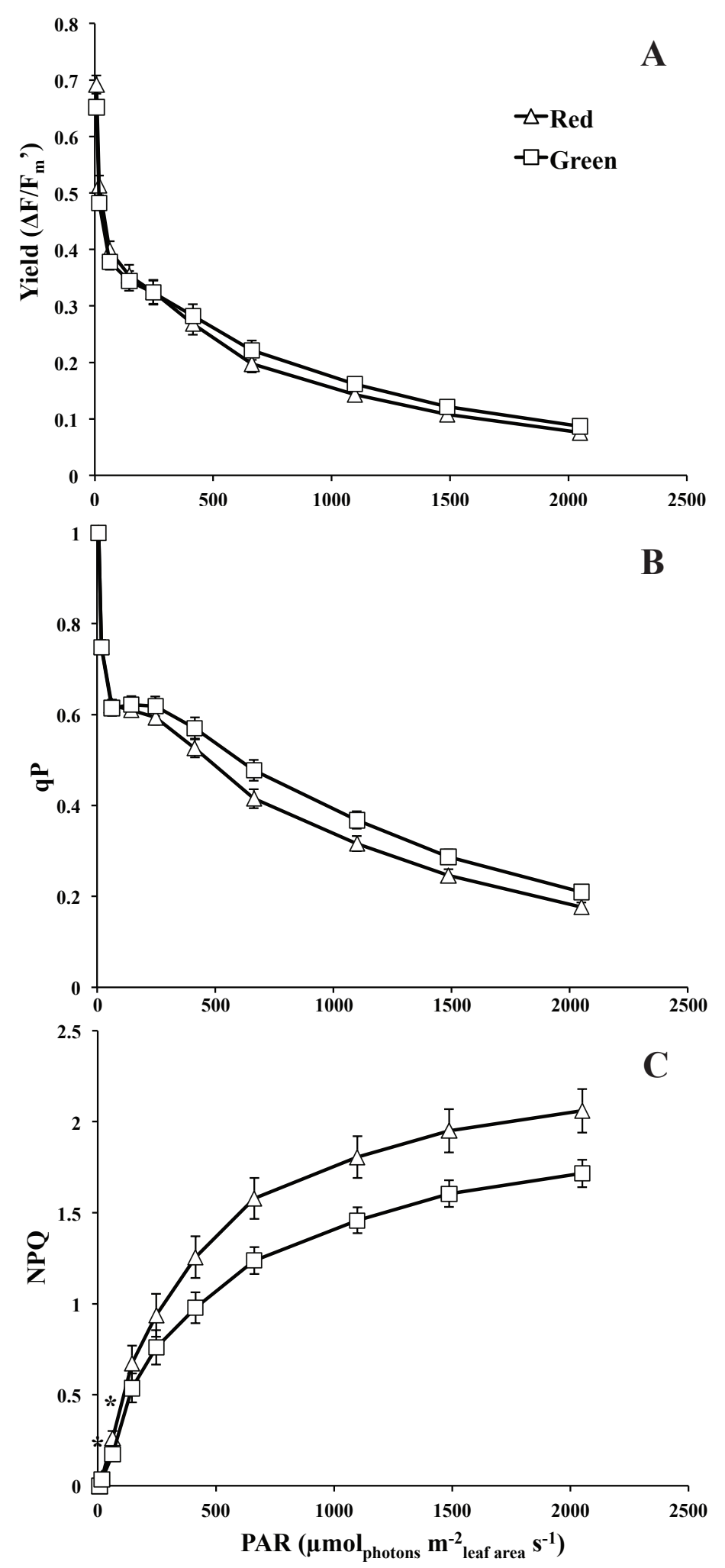

Fig. 4.5 Light response curves for (A) photochemical quantum yield $\left(\Delta F / F_{\mathrm{m}}{ }^{\prime}\right),(\mathbf{B})$ photochemical quenching $(q P)$, and $(\mathbf{C})$ non-photochemical quenching $(N P Q)$ of photosystem II for matched red and green leaves of $P$. colorata. Means $\pm \mathrm{SE}, \mathrm{n}=10$ pairs. 


\subsubsection{Photoinhibition of red and green P. colorata leaves}

The white saturating light treatment caused a highly significant reduction in the $F_{\mathrm{v}} / F_{\mathrm{m}}$ of leaves $\left(\mathrm{F}_{1,9}=560, P<0.001, \eta^{2}=0.96\right)$. There was no statistical difference in $F_{\mathrm{v}} / F_{\mathrm{m}}$ between dark-adapted matched red and green $P$. colorata leaves, nor after the saturating light treatment $\left(\mathrm{F}_{1,9}=0.16, P=0.7, \eta^{2}=0\right)$. There was an extremely weak but significant interaction, as dark-adapted red leaves appeared to have the marginally higher dark adapted $F_{\mathrm{v}} / F_{\mathrm{m}}$, yet experienced a slightly larger decline in $F_{\mathrm{v}} / F_{\mathrm{m}}$ after photoinhibition $\left(\mathrm{F}_{1,9}=5.3, P=0.047, \eta^{2}=0.004\right.$, Fig. 4.6).

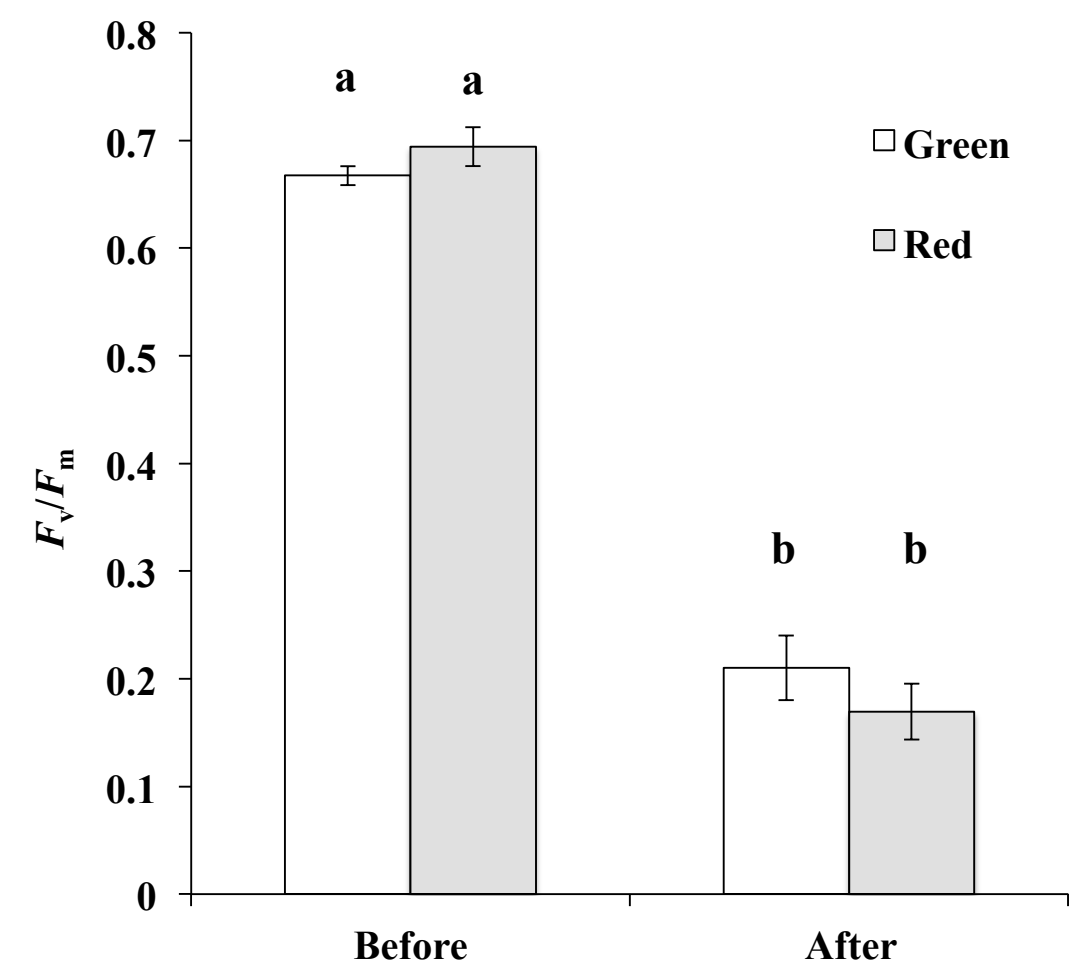

Fig. 4.6 $F_{\mathrm{v}} / F_{\mathrm{m}}$ of matched red and green $P$. colorata leaves before (dark-adapted for $4 \mathrm{~h}$ ) and after exposure to $2000 \mu \mathrm{mol} \mathrm{m} \mathrm{m}^{-2} \mathrm{~s}^{-1}$ of collimated white light for $0.5 \mathrm{~h}$ (means $\pm \mathrm{SE}, \mathrm{n}=10$ pairs). Different letters indicate statistical significance (repeated measures ANOVA, $P<0.05)$.

\subsubsection{Photoinhibition of red and green P. colorata leaves under white and red/blue lights}

Regardless of lamp colour, the saturating light treatment caused a highly significant depression in the $F_{\mathrm{v}} / F_{\mathrm{m}}$ of $P$. colorata leaves $\left(\mathrm{F}_{1,9}=2000, P<0.001, \eta^{2}=0.93\right)$. However there was no statistical difference in the magnitude of the decline between 
the different light colour treatments $\left(\mathrm{F}_{1,9}=0.09, P=0.77, \eta^{2}=0\right)$, nor between different leaf colours within those treatments $\left(\mathrm{F}_{1,9}=3, P=0.101, \eta^{2}=0\right)$. There were no significant interactions between leaf colour, light colour or saturating light treatments was significant (Fig. 4.7).

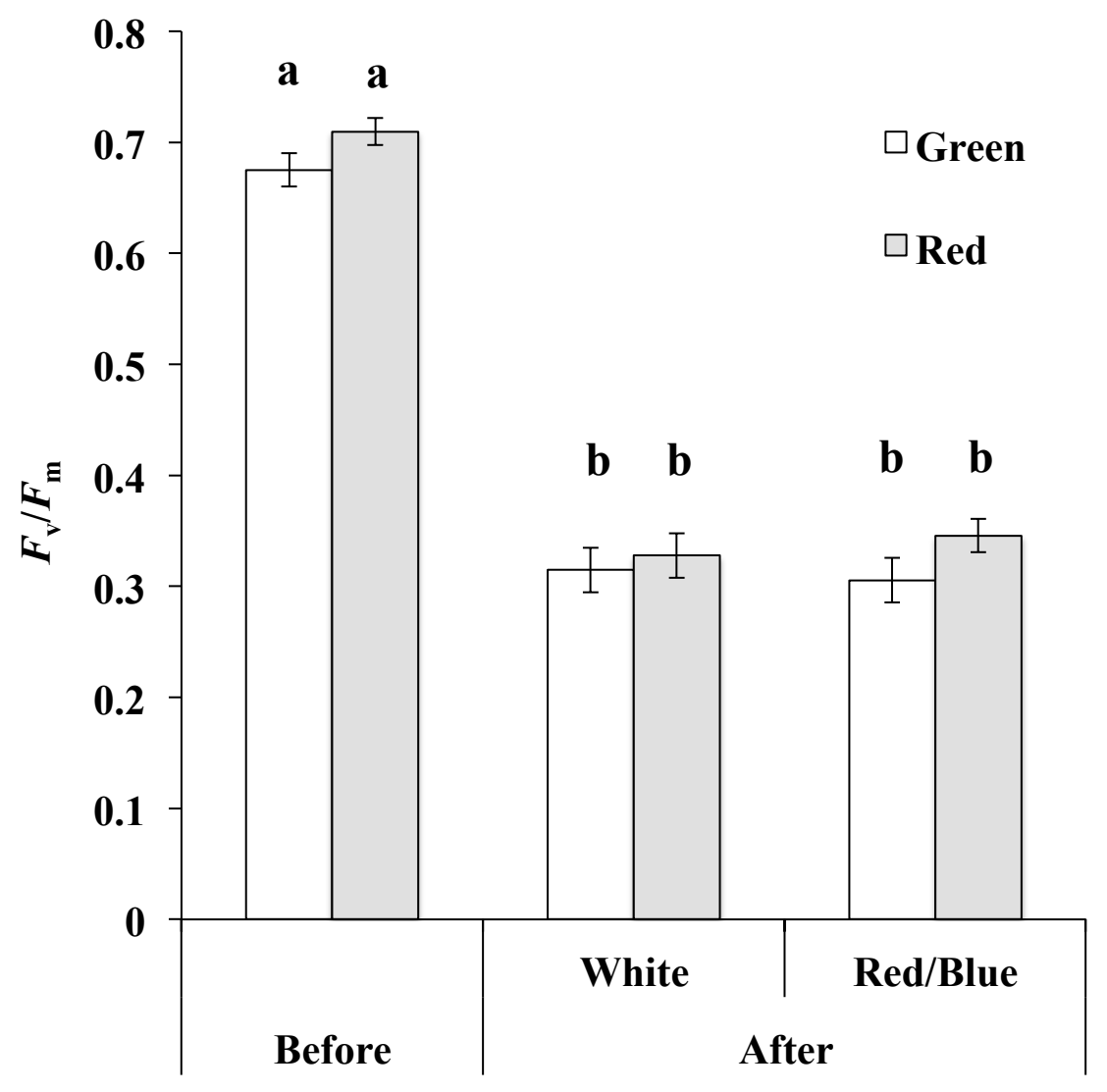

Fig. $4.7 F_{\mathrm{v}} / F_{\mathrm{m}}$ of matched red and green $P$. colorata leaves before (dark-adapted for $24 \mathrm{~h}$ ) and after exposure to $2000 \mu \mathrm{mol} \mathrm{m} \mathrm{m}^{-2} \mathrm{~s}^{-1}$ of either white or red/blue light for 0.5 h. Means \pm SE, $\mathrm{n}=10$ pairs. Different letters indicate statistical significance (repeated measures ANOVA, $P<0.05)$.

\subsection{DISCUSSION}

Given that red pigments in $P$. colorata leaves may function for signalling (see Chapter 2), I investigated the consequences to photosynthesis of maintaining anthocyanin pigments within leaves. My results indicate that the presence of anthocyanin pigments within mature, non-senescing leaves may incur a photosynthetic cost. Redder P. colorata leaves in the field had over $30 \%$ lower maximum assimilation rate than matched green leaves from neighbouring trees 
(paired Student's $t$-test, $\mathrm{n}=9$ pairs, $t=2.755, \mathrm{P}=0.025$, Fig. 4.3). There was no measurable advantage in terms of photoprotection in the red P. colorata leaves (Fig. 4.6 \& Fig. 4.7).

The rates of $\mathrm{CO}_{2}$ assimilation found in this study were comparable with those reported for other New Zealand forest tree species (Gould et al. 2002b, Tissue et al. 2005). Leaves from green $P$. colorata trees had a higher light saturated $\mathrm{CO}_{2}$ assimilation rate $\left(A_{\mathrm{sat}}\right)$ than their matched red trees (Fig. $4.2 \&$ Fig. 4.3). This result is consistent with other studies using intraspecific comparisons of $\mathrm{CO}_{2}$ assimilation rates among mature, non-senescing, red and green leaves (Gould et al. 2002b, Nielsen and Simonsen 2011, Nikiforou et al. 2011, Zhang et al. 2011). While statistically significant, the differences in light saturated $\mathrm{CO}_{2}$ assimilation between red and green matched leaves found in this study were quantitatively small $(\mathrm{n}=9$ pairs, red $=3.2 \pm$ 0.45 , green $=4.7 \pm 0.67$, Mean $\pm \mathrm{SE}, \mu \mathrm{mol}_{\mathrm{CO}_{2}} \mathrm{~m}_{\text {leaf area }}^{-1} \mathrm{~s}^{-1}$, Fig. 4.3). Additionally, at subsaturating light fluxes, I found no differences between red and green leaves. The trees would not experience saturating light fluxes all day, so the actual difference in productivity would be less than that indicated by the differences in $A_{\text {sat }}$. Regardless, if these small leaf level differences in photosynthesis scale up to whole-plant level differences, a reduction in photosynthesis could potentially lead to differences in relative growth and reproduction within matched pairs of red and green $P$. colorata trees. Losses in photosynthetic achievement have been shown to result in impaired plant fitness (Ganeteg et al. 2004). Therefore at low levels of herbivory, when the benefits of signalling are similar to the cost of photosynthesis, one would expect equal fitness payoffs for red and green P. colorata trees. This is consistent with the results seen in Chapter 2.

The lower photosynthetic rate of red $P$. colorata leaves does not appear to be a result of attenuation of light by foliar anthocyanin pigments. Contrary to other published studies comparing red and green leaves (Manetas et al. 2003, Hughes and Smith 2007b, Kyparissis et al. 2007, Zeliou et al. 2009, Nikiforou and Manetas 2010), the pigment profiles of red $P$. colorata leaves do not resemble those of shade-adapted green leaves. Although red $P$. colorata leaves contained significantly lower chlorophyll $a$, they also contained less chl $b$ (although not statistically significant) than green leaves. As a result, there was no difference in the chlorophyll $a: b$ ratio 
between matched red and green P. colorata leaves (Fig. $4.4 \&$ Table 4.3). The reduced $A_{\text {sat }}$ of red leaves could be a result of photochemical constraints limiting the maximum rate of photosynthetic carbon metabolism relative to leaves from green plants (e.g. smaller light harvesting complexes, and lower levels of rubisco). Alternatively, as the red pigmentation in $P$. colorata leaves is often patchily distributed over the leaf lamina, perhaps the chl $a: b$ ratios underneath red portions of $P$. colorata leaves are consistent with shading, yet vary at a smaller spatial scale than was detectable by my analyses. Another possible interpretation is that the higher light saturated $\mathrm{CO}$ 
anthocyanin pigments either (Esteban et al. 2008, Liakopoulos and Spanorigas 2012). Given that anthocyanin pigments in leaves with irregular patches and variegation appear to not primarily function for photoprotection, non-green colouration in these leaves may be more likely to function for the purpose of changing leaf appearance (see 'Biotic hypotheses for foliar anthocyanins' section 1.5.3). The lack of support for the photoprotection hypothesis presented in this chapter lends indirect support for a signalling function for anthocyanins in $P$. colorata leaves. 
Table 4.4 A list of studies classified as either in support of, equivocal, or against a photoprotective function for anthocyanin pigments.

\begin{tabular}{clll}
\hline Photoprotection & Study & Species & Comparison \\
\hline For & Gould et al. (1995) & Begonia pavonina \& Triolena & Green vs abaxially red leaves (intraspecies) \\
& hirsute & \\
& Mendez et al. (1999) & Pinguicula vulgaris & Green vs UV-B induced red (carnivorous plant) \\
& Manetas et al. (2002) & Rosa sp. \& Ricinus communis & Green mature vs red juvenile \\
& Neill and Gould (2003) & Lactuca sativa & Green vs red portions of leaves \\
& Manetas et al. (2003) & Quercus coccifera & Green vs red juvenile leaves \\
& Liakopoulos et al. (2006) & Vitis vinifera & Green vs transiently red cultivars \\
& Hughes and Smith (2007a) & Galax urceolata & Green vs winter-red leaves \\
& Hughes et al. (2005) & Galax urceolata & Green vs winter-red leaves \\
& Hughes (2012a) & 10 broadleaf evergreen spp. & Green vs winter-red (interspecies) \\
& Hughes et al. (2007) & Acer rubrum, Cercis & Correlation between anthocyanin reassimilation and leaf \\
& canadensis \& Liquidambar & photosynthetic development (intraspecies) \\
& styraciflua & Pyrus communis & Green vs red fruit peel \\
\hline
\end{tabular}


Table 4.4 Continued

\begin{tabular}{|c|c|c|c|}
\hline Photoprotection & Study & Species & Comparison \\
\hline \multirow[t]{12}{*}{ For } & Steyn et al. (2009) & $\begin{array}{l}\text { Malus domestica \& Pyrus } \\
\text { communis }\end{array}$ & $\begin{array}{l}\text { Intraspecies correlation between photoprotection and redness } \\
\text { (fruit peel) }\end{array}$ \\
\hline & Fondom et al. (2009) & Agave striata & Young leaves from green and red morphs (CAM plant) \\
\hline & Novak and Short (2011) & Thalassia testudinum & Green vs red leaves (seagrass) \\
\hline & Nielsen and Simonsen (2011) & Oxalis triangularis & Green vs red varieties \\
\hline & Zhang et al. (2012) & Arabidopsis & Green anthocyanin-deficient mutant vs wild type \\
\hline & Pietrini and Massacci (1998) & Zea mays L. & Green vs cold-induced anthocyanic leaves \\
\hline & Pietrini et al. (2002) & Zea mays $\mathrm{L}$. & Green vs red genotypes \\
\hline & Feild et al. (2001) & Cornus stolonifera & Yellow vs red senescing leaves \\
\hline & Gould et al. (2010) & $\begin{array}{l}\text { Cornus stolonifera }+ \text { other } \\
\text { species }\end{array}$ & $\begin{array}{l}\text { Green vs red stems }+ \text { interspecies correlation between } \\
\text { photoprotection and stem anthocyanin content }\end{array}$ \\
\hline & Krol et al. (1995) & Pinus banksiana Lamb. & Correlation between photoprotection and anthocyanin content \\
\hline & $\begin{array}{l}\text { Smillie and Hetherington } \\
\text { (1999) }\end{array}$ & Bauhunua variegata & Green vs red/purple pods \\
\hline & Hoch et al. (2003) & $\begin{array}{l}\text { Cornus sericea, Vaccinium } \\
\text { elliottii, \& Viburnum sargentii }\end{array}$ & Anthocyanin-deficient mutant vs wild type (intraspecies) \\
\hline
\end{tabular}


Table 4.4 Continued

\begin{tabular}{|c|c|c|c|}
\hline Photoprotection & Study & Species & Comparison \\
\hline \multirow[t]{5}{*}{ No Difference } & Burger and Edwards (1996) & Coleus & Green vs. red varieties \\
\hline & Kytridis et al. (2008) & Cistus creticus & Green vs winter-red \\
\hline & $\begin{array}{l}\text { Liakopoulos and Spanorigas } \\
\text { (2012) }\end{array}$ & Pelargonium $\times$ hortorum & Green vs red portions of variegated leaves \\
\hline & Lee et al. (2003) & 18 deciduous tree spp. & Yellow vs red senescing leaves (interspecies) \\
\hline & $\begin{array}{l}\text { Karageorgou and Manetas } \\
(2006)\end{array}$ & Quercus coccifera & Green vs red individuals \\
\hline \multirow[t]{6}{*}{ Against } & Dodd et al. (1998) & Syzygium spp. & $\begin{array}{l}\text { Green vs red juvenile leaves (interspecies) [although red } \\
\text { leaves had lower photosynthetic capacity] }\end{array}$ \\
\hline & Kyparissis et al. (2007) & $\begin{array}{l}\text { Chimeric Prunus domestica } \mathrm{L} \text {. } \\
+ \text { P. cerasifera }\end{array}$ & P. domestica L. (green leaves) vs $P$. cerasifera (red leaves) \\
\hline & Zeliou et al. (2009) & Cistus creticus & Green vs winter-red \\
\hline & $\begin{array}{l}\text { Nikiforou and Manetas } \\
\text { (2010) }\end{array}$ & Pistacia lentiscus & Green vs red \\
\hline & Esteban (2008) & E. dens-canis & Green vs red portions of variegated leaf \\
\hline & Lan $(2011)$ & Fagopyrum dibotrys & Green vs red-mutant juvenile leaves \\
\hline
\end{tabular}


In this study, data from chlorophyll fluorescence light curves may preclude the conclusion that the observed lack of difference in $\Delta F_{\mathrm{v}} / F_{\mathrm{m}}$ between red and green leaves under white light rules out a photoprotective role for anthocyanin pigments in P. colorata (Fig. 4.6 \& Fig. 4.7). To conclude that foliar anthocyanins serve as photoprotectants requires that my photoinhibitory treatments did indeed provide sufficient stress to exceed the capacity of $P$. colorata leaves to dissipate excess light energy. The plant material used in this experiment came from a very exposed ridgetop field site that regularly experiences extreme wind speeds, low temperatures and bright sunlight. As a result, these trees are likely to have been well acclimated to such conditions. Rapid chlorophyll fluorescence light curves, carried out on leaves that had been dark-adapted for $4 \mathrm{~h}$, showed that levels of $N P Q$ for both red and green leaves did not saturate, even at irradiances exceeding $2000 \mu \mathrm{mol} \mathrm{m}^{-2} \mathrm{~s}^{-1}$ (Fig. 4.5B \& C). Furthermore, the leaves used in the white vs. red/blue photoinhibition experiment were dark-adapted for $12 \mathrm{~h}$, three times longer than the leaves for which $N P Q$ was measured, which may have allowed for more restoration of slower reversible $N P Q$ processes (Demmig-Adams and Adams III 1996, Maxwell and Johnson 2000, Hughes et al. 2012a). In support of this, $P$. colorata leaves that were dark-adapted for $12 \mathrm{~h}$ had a smaller decline in $F_{\mathrm{v}} / F_{\mathrm{m}}$ after photoinhibition with white light, than those that were dark-adapted for $4 \mathrm{~h}$ (compare Fig. 4.6 \& Fig. 4.7). To more comprehensively assess the photoprotective influence of foliar anthocyanin pigments, future experiments should combine cold and light stress (Henriques 2009, Gould et al. 2010), use a white light source that better approximates the solar spectrum for visible radiation, a longer duration photoinhibitory treatment, and possibly include the use of antioxidant assays (Neill et al. 2002a).

Consistent with many other studies of anthocyanins, red $P$. colorata leaves had higher levels of $N P Q$ than green (Kyparissis et al. 2007, Li et al. 2009, Gould et al. 2010, Lan et al. 2011, Zhang et al. 2011, Hughes et al. 2012a). NPQ is a measure of the efficiency with which a leaf is able to dissipate excess light energy as heat (Maxwell and Johnson 2000, Demmig-Adams and Adams III 2006). The major mechanism for the dissipation of excess energy occurs by the action of the carotenoid pigment xanthophyll cycle, a reversible process where excess light energy drives the build-up of a trans-thylakoid $\mathrm{pH}$ gradient that in turn drives the conversion of violaxanthin to zeaxanthin, via the intermediate antheraxanthin (Demmig-Adams and Adams III 
1996). However, all the studies that have compared the xanthophyll cycle pool sizes of red and green leaves (both inter- and intraspecies comparisons) have found that red leaves have lower concentrations of violaxanthin + antheraxanthin + zeaxanthin per unit chlorophyll (VAZ/Chl) than green leaves (Pietrini et al. 2002, Liakopoulos et al. 2006, Kytridis et al. 2008, Zeliou et al. 2009, Nikiforou et al. 2010, Nielsen and Simonsen 2011, Hughes et al. 2012a). I did not detect any difference in either the FW concentrations of total carotenoids, or the ratio of carotenoid to chlorophyll concentrations between red and green matched P. colorata leaves (Table 4.3). However, there are many different carotenoid pigments within leaves, and measuring the total concentration of carotenoids may be a poor estimation of xanthophyll pigment concentrations. To more thoroughly understand the photochemical efficiency and heat dissipation capacity of red and green $P$. colorata leaves, a detailed assessment of the diurnal changes in photochemistry, $F_{\mathrm{v}} / F_{\mathrm{m}}, N P Q$ and the deepoxidation state of xanthophyll cycle pigments is needed (Maxwell and Johnson 2000, Hughes et al. 2012a). 


\section{Feeding and oviposition preference of the insect herbivores of $P$. colorata}

\subsection{ABSTRACT}

The leaf signalling hypothesis predicts that plants use non-green leaf colouration as a visual signal to communicate their defensive capacity to approaching herbivores, who consequently avoid those well-defended plants. To test whether the leaf signalling hypothesis explains leaf colour variation in different systems, a suitable herbivore must be identified. I used field surveys and laboratory bioassays to identify which invertebrate herbivores are most likely to participate in coevolved leaf signalling interactions with $P$. colorata. I then tested the hypothesis that herbivores prefer to eat green than red $P$. colorata leaves. Feeding preference bioassays showed that brownheaded leafrollers (C. obliquana and C. herana) and Auckland tree weta (Hemideina thoracica) preferentially consumed green rather than red $P$. colorata leaves. Gravid female $C$. obliquana moths did not exhibit any oviposition preference for leaves of either colour. Results from these bioassays, combined with my field surveys suggest that Ctenopseustis spp. leafroller larvae are a likely coevolution partner for P. colorata.

\subsection{INTRODUCTION}

The leaf signalling hypothesis predicts that trees use non-green leaf colouration as a visual signal to communicate their defensive capability to herbivores, which consequently avoid those well-defended plants (Archetti 2000, Hamilton and Brown 2001, Archetti and Brown 2004). When developing this hypothesis, Hamilton and Brown (2001) proposed that the bright colouration of senescing leaves in autumn is a product of coevolution between deciduous trees and those aphid species whose hostselecting flights coincide with the timing of leaf senescence. There is growing evidence from patterns of plant colouration and herbivory in the field, that this hypothesis may also explain red leaf colouration in some non-senescing leaves (Wong and Srivastava 2011, Cooney et al. 2012, Youard 2012). However the identities and characteristics of the putative herbivore coevolution partners of these non-senescing red-leafed plant species have not been thoroughly investigated. 
Hamilton and Brown (2001) discussed the ideal herbivore characteristics that would most likely result in a coevolved plant-herbivore signalling system. The herbivore should: $a$ ) have a long history of association with the host plant; $b$ ) be mobile enough to travel among host plant individuals at the time that the colouration is displayed; $c$ ) injure the host plant - the more damaging the insect herbivore, the greater is the selective pressure driving the evolution of leaf signalling; $d$ ) be discriminating when presented with host individuals of different quality, preferring the less defended/more suitable host trees; and $e$ ) process visual stimuli to achieve this discrimination. For the proposed autumn-leaf/aphid signalling system, there is evidence that some species of aphids may fulfil these requirements (Archetti and Leather 2005, Ramirez et al. 2008, Archetti 2009b, Döring et al. 2009). To test whether the leaf signalling hypothesis explains leaf colour variation in non-senescing red-leafed plant species, a suitable herbivore that fulfils all of the above requirements must be identified.

Hamilton and Brown (2001) focused on coevolution between a plant host species and its specialist herbivores, citing Coley and Barone (1996) who argued that specialist herbivores are more damaging. However, this does not necessarily preclude generalist herbivores provided they are sufficiently damaging, and regularly abundant.

Examples of tight pairwise co-evolution between plants and insect herbivores are rare (Stamp 2003). Additionally, specialist herbivores can evolve mechanisms to circumvent the chemical defences of their hosts (Bowers and Puttick 1988, Dobler et al. 2011). This would reduce the selective pressure for herbivores to discern between differently defended host individuals. Searches for appropriate herbivores need not be restricted to specialist monophages.

The non-senescing leaves of the New Zealand (NZ) endemic Pseudowintera colorata exhibit patterns in colour, chemistry and herbivory consistent with the plant component of the leaf signalling hypothesis: redder leaves are more well-defended and incur less chewing herbivory than equivalent greener leaves (Cooney et al. 2012, Youard 2012), but a thorough investigation into the characteristics of $P$. colorata's herbivores has not been carried out in the context of leaf signalling. I used literature searches and surveys of field populations to assess which NZ endemic insect herbivore species have a long historical association with, and are abundant upon, $P$. 
colorata. From these species, given that red $P$. colorata leaves are better defended against insects, I carried out feeding preference trials to assess whether they consumed $P$. colorata and, if they did, their ability to discriminate between green and predominantly red $P$. colorata leaves. For the species with flying adult life forms, I tested the ability of gravid females to discriminate between red and green leaves.

\subsection{MATERIALS AND METHODS}

\subsubsection{Herbivore survey}

Potential candidate herbivores were selected using a list of known herbivores (http://plant-synz.landcareresearch.co.nz/; accessed August 2010), plus day and night time surveys of $P$. colorata in the field. Both visual surveys and branch-beating methods (Majer et al. 1996, Sutherland 2006) were used to census chewing herbivores on 20 P. colorata plants at each field site. The field sites, both located in the lower North Island of New Zealand, represented the range of typical habitats and growth forms of $P$. colorata (Norton 1980, Cooney et al. 2012); a stunted shrub-like population forming a consolidated canopy on an exposed ridgetop (Belmont trig, Belmont Regional Park, Wellington, $41^{\circ} 11^{\prime} 0.2^{\prime}$ 'S, $174^{\circ} 52^{\prime} 25.9^{\prime}$ 'E) and a population of understory trees $(<3 \mathrm{~m}$ height $)$ in high $(800 \mathrm{~m})$ montane forest dominated by Nothofagus spp. (Otaki Forks, Tararua Forest Park, 4054'27.8'’S, 175¹5'21.1'’E). In addition to the surveys, I applied Tanglefoot ${ }^{\mathrm{TM}}$ (Contech Enterprises Inc., Victoria, BC, Canada) to 10 pairs of matched red and green P. colorata at Belmont trig, to trap any flying insects that landed upon leaves. Three stems were randomly selected from each plant and Tanglefoot was applied to the adaxial and abaxial surfaces of three fully expanded leaves near the apex of each stem. Leaves and captured insects were retrieved after one week.

\subsubsection{Insect material}

For the bioassays, three species of lepidopteran larvae were obtained from lab colonies maintained by The New Zealand Institute for Plant \& Food Research, Auckland: two species of brownheaded leafroller caterpillars, Ctenopseustis obliquana (Lepidoptera: Tortricidae: Tortricinae) and C. herana, and the common forest looper Pseudocorremia suavis (Lepidoptera: Geometridae: Ennominae). All 
had been raised in captivity on an artificial diet (Singh 1983). For fitness and oviposition trials, C. obliquana eggs and pupae were also provided by The New Zealand Institute for Plant \& Food Research, Auckland.

Two female adult Auckland tree weta, Hemideina thoracica (Orthoptera), were collected from a forest reserve near Levin at the southernmost limits of their distribution. G. W. Gibbs provided five Wellington tree weta, H. crassidens (Orthoptera) from weta hotels in his own garden. Two female stick insects, Acanthoxyla prasine speciosa (Phantasmodea), were collected from P. J. Lester's garden in Wellington NZ.

\subsubsection{Plant material}

Leaf material for the oviposition and feeding preference bioassays was selected from nursery-grown potted $P$. colorata plants ecosourced from the central North Island of New Zealand. Leaves from $P$. colorata plants from this region have low levels of the sesquiterpene dialdehyde 9-deoxymuzigadial (Wayman et al. 2010), similar to plants from the Otaki Forks field site (Cooney et al. 2012). The potted plants were divided into two groups consisting of plants with either predominantly red or predominantly green leaves.

Leaf material for the second oviposition bioassay and the insect fitness experiments was collected from $P$. colorata near Belmont trig. The Belmont population of $P$. colorata is typical of the 'mixed chemotype' described by Wayman et al. (2010), having similar levels of the two sesquiterpene dialdehydes 9-deoxymuzigadial and polygodial.

\subsubsection{Feeding preference bioassays}

With assistance of Loïc Cizabuiroz (visiting French student), I undertook bioassays to assess the ability of the candidate herbivores species to discriminate between red and green $P$. colorata leaves. I carried out feeding preference bioassays on all of the chewing herbivores observed during surveys of my field sites, with the addition of Auckland tree weta (Hemideina thoracica) as it is known to consume P. colorata (Barrett 1991). Trials were conducted in a glasshouse to provide natural illumination. 
Caterpillar trials were conducted in clear plastic petri dishes (0.09 $\mathrm{m}$ diameter) (Fig. 5.1A). For weta, trials were conducted in $2 \mathrm{~L}$ plastic containers with wire mesh insets in their lids (Fig. 5.1B). I experimented with several containers for stick insects, including $2 \mathrm{~L}$ containers and glass aquaria (Fig. 5.1C). Humidity within the experimental containers was maintained by a folded section of moist paper towel. In the wild, during daylight hours tree weta roost inside cavities in tree trunks (Trewick and Morgan-Richards 1995). To replicate this, weta were provided with wooden artificial roosts. Due to the low numbers of $H$. thoracica and Acanthoxyla sp., I carried out four replicate bioassays per individual insect.

Pairs of fully expanded leaves, one green and one predominantly red, were selected from the potted P. colorata seedlings. Within pairs, leaves were matched as closely as possible for size, shape, and leaf age. Leaves with any evidence of mechanical damage or herbivory were rejected. Leaves were excised, imaged using a Canon CanoScan 8400F flatbed scanner (Tokyo, Japan), and placed in pairs within the containers. Leaf position was alternated to control for orientation biases. For caterpillar trials, one insect was placed in the middle of each petri dish, equidistant from both leaves (Fig. 5.1A). For weta trials, leaves were placed equidistant to the opening of their artificial logs (Fig. 5.1B). Trials were run for $72 \mathrm{~h}$, after which the leaves were scanned once again.

Several methods of leaf presentation were trialled for stick insects. Leaves were initially placed inside $2 \mathrm{~L}$ plastic containers similar to the weta trials. Trials were repeated with leaves suspended from the lid of the containers. Finally, P. colorata leaves were attached with tape to otherwise leafless divaricating stems within two glass aquaria (Fig. 5.1C). It was thought that this, more tree-like method of leaf presentation, might promote herbivory. 


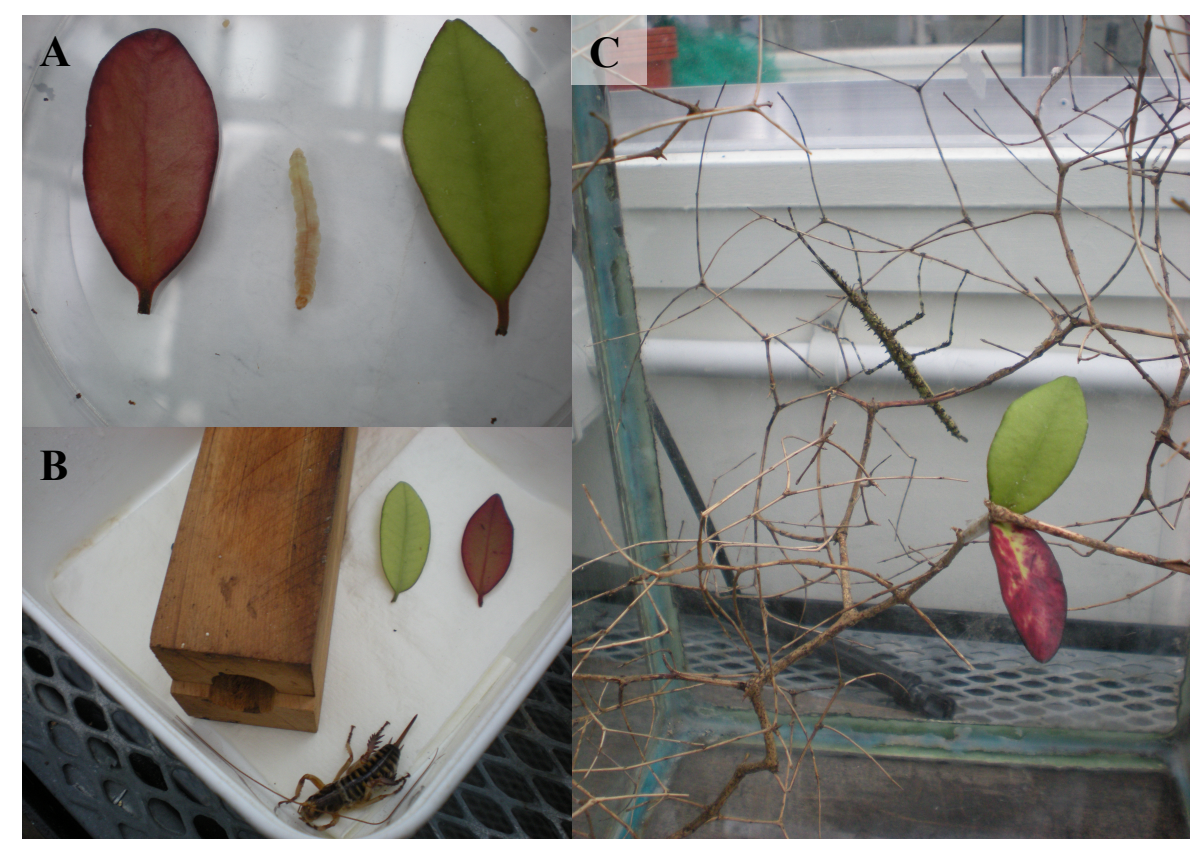

Fig. 5.1 Photographs of feeding preference bioassays for caterpillars (A), weta (B), and stick insects $(\mathbf{C})$.

All insects were starved for $24 \mathrm{~h}$ before each trial. Leading up to, and after the bioassays, weta were provided with pieces of carrot (Barrett 1991), stick insects were given pōhutukawa (Metrosideros excelsa) leaves (Salmon 1991) and caterpillars were kept in $5 \mathrm{~mL}$ test tubes containing general purpose diet (Singh 1983).

\subsubsection{Leaf area analysis}

Scanned images were used to quantify the area of leaf tissue removed by herbivory. Initial leaf area and post-herbivory leaf area were measured using ImageJ v1.41 (National Institutes of Health, Bethesda, MD, USA). To account for small differences in initial leaf area, the amount of feeding damage was calculated as the percentage of initial leaf area removed.

\subsubsection{Oviposition experiments}

I used two methods to assess the oviposition preferences of gravid female Lepidoptera. One, following the methods of Cooney et al. (2012), employed 59 pots (18 cm tall $\times 20 \mathrm{~cm}$ in diameter) with sandpaper-lined sides, nylon mesh covering and 
vermiculite covered base, each containing one red and one green excised $P$. colorata leaf. These pots were set up inside a rooftop glasshouse to receive natural illumination. The leaves were presented at a $45^{\circ}$ angle from the ground with the petiole in a shallow dish of water. After they had emerged from pupae, I introduced one male and one female Ctenopseustis obliquana moth to each pot. After $72 \mathrm{~h}$, each leaf was inspected under a dissecting microscope, and the presence or absence of egg masses was recorded.

The second method used 20 plastic petri dishes ( $0.09 \mathrm{~m}$ diameter) with loose fitting lids. To prevent leaf desiccation, I placed the petiole of each leaf through a small hole in the lid of a water-filled $1.5 \mathrm{~mL}$ microcentrifuge tube, and sealed around the petiole with parafilm. One female and one male $C$. obliquana pupa, and $1.5 \mathrm{~cm}^{2}$ moistened paper towel, were placed into each petri dish. The petri dishes were placed within an incubator at 18:6 h (light:dark) photoperiod, $\sim 60 \%$ relative humidity $(\mathrm{RH}), 20{ }^{\circ} \mathrm{C}$ (Lester and Barrington 1997), and regularly monitored for five days. Afterwards, each leaf was inspected under a dissecting microscope and the presence or absence of egg masses was recorded.

\subsubsection{Statistical analyses}

For the feeding preference trials, paired $t$-tests were used to compare the $\%$ leaf area removed from red and green leaves. After Shapiro-Wilk tests for normality, herbivory preference of each species of insect was analysed using a nonparametric Wilcoxon signed rank test. Oviposition preference (presence of eggs on red, green or both leaves) was analysed using a chi-squared test. All analyses were carried out in PSAW/SPSS Version 18.0 (Chicago, IL, USA).

\subsection{RESULTS}

\subsubsection{Herbivore survey}

For the plants growing at the two field sites, 10 taxa of invertebrates were noted on their foliage (Table 5.1). 
Table 5.1 List of species observed on P. colorata at two fieldsites in the lower North Island of New Zealand.

\begin{tabular}{|c|c|c|c|c|}
\hline Fieldsite & Method & Species & No. observed & Notes \\
\hline \multirow[t]{6}{*}{ Otaki Forks } & Night & Green leaf slug (Gastropoda: & 8 & Not folivorous, eat algae and fungi off leaves (G. W. Gibbs pers. comm.). \\
\hline & & Athoracophoridae) & & \\
\hline & & Weta nymphs & 5 & Weta nymphs difficult to identify in the field (G. W. Gibbs pers. comm.). \\
\hline & & Tree weta (presumably H. crassidens) & $>1$ & Heard, not seen. \\
\hline & Day & Aphid (Hemiptera) & 1 & Evaded capture. \\
\hline & & $\begin{array}{l}\text { Brownheaded leafroller larvae } \\
\text { (Ctenopseustis spp.) }\end{array}$ & 25 & Found within leaf shelters, with abundant signs of recent herbivory. \\
\hline \multirow[t]{9}{*}{ Belmont trig } & Night & Tree weta (presumably H. crassidens) & 1 & Heard, not seen. \\
\hline & Day & Scale insect (Ctenochiton spp.) & 5 & Light green on abaxial leaf surface, causes raised leaf deformations. \\
\hline & & Wellington tree weta $(H$. crassidens $)$ & $\sim 10$ & Saw $\sim 10$ in three years of fieldwork \\
\hline & & Stick insect (Phantasmodea) & 16 & $\begin{array}{l}\text { First summer of fieldwork observed } 8 \text { pairs of mating stick insects walking on } P \text {. } \\
\text { colorata, no subsequent sightings. }\end{array}$ \\
\hline & & $\begin{array}{l}\text { Brownheaded leafroller larvae } \\
\text { (Ctenopseustis spp.) }\end{array}$ & 45 & Observed within leaf shelters, and actively building leaf shelters. \\
\hline & & $\begin{array}{l}\text { Common forest looper larvae } \\
\text { (Pseudocorremia spp.) }\end{array}$ & 1 & \\
\hline & Tanglefoot & Flies (Diptera: Calliphoridae) & & Not folivorous. \\
\hline & & Crane flies (Diptera: Tipulidae) & & Not folivorous. \\
\hline & & Various spiders & & Not folivorous. \\
\hline
\end{tabular}




\subsubsection{Feeding preference bioassays}

Both species of brownheaded leafroller larvae discriminated between red and green $P$. colorata leaves, removing on average $\sim 6 \%$ more leaf area from green than red leaves (C. obliquana, $\mathrm{Z}=-2.2, P=0.027 ; C$. herana, $\mathrm{Z}=-2.7 P=0.007$, Fig. 5.2, Table 5.2). The common forest looper Pseudocoremia suavis did not discriminate between red and green $P$. colorata leaves $(\mathrm{Z}=-0.676, P=0.499$, Fig. 5.2). In the two occasions that $P$. suavis larvae chewed both leaves, one ate more from the green leaf while the other ate more from the red leaf.

Table 5.2 Frequency of herbivory by Ctenopseustis spp. leafrollers of both green and red, either green or red, or neither P. colorata leaf in feeding preference bioassays.
C. obliquana
C. herana

\section{Consumed both leaves}

Preferred green

Preferred red

\section{Consumed only one leaf}

Chose green

Chose red

Did not consume either leaf
5

2

6

2

2
2

1

3

11

Of the large chewing herbivores, only $H$. thoracica browsed any $P$. colorata leaf material, consuming on average $25 \%$ more leaf area from green than red leaves $(\mathrm{Z}=-2.1, P=0.036)$. Neither $H$. crassidens nor $A$. prasina speciosa consumed any $P$. colorata during the trials (Fig. 5.2). 


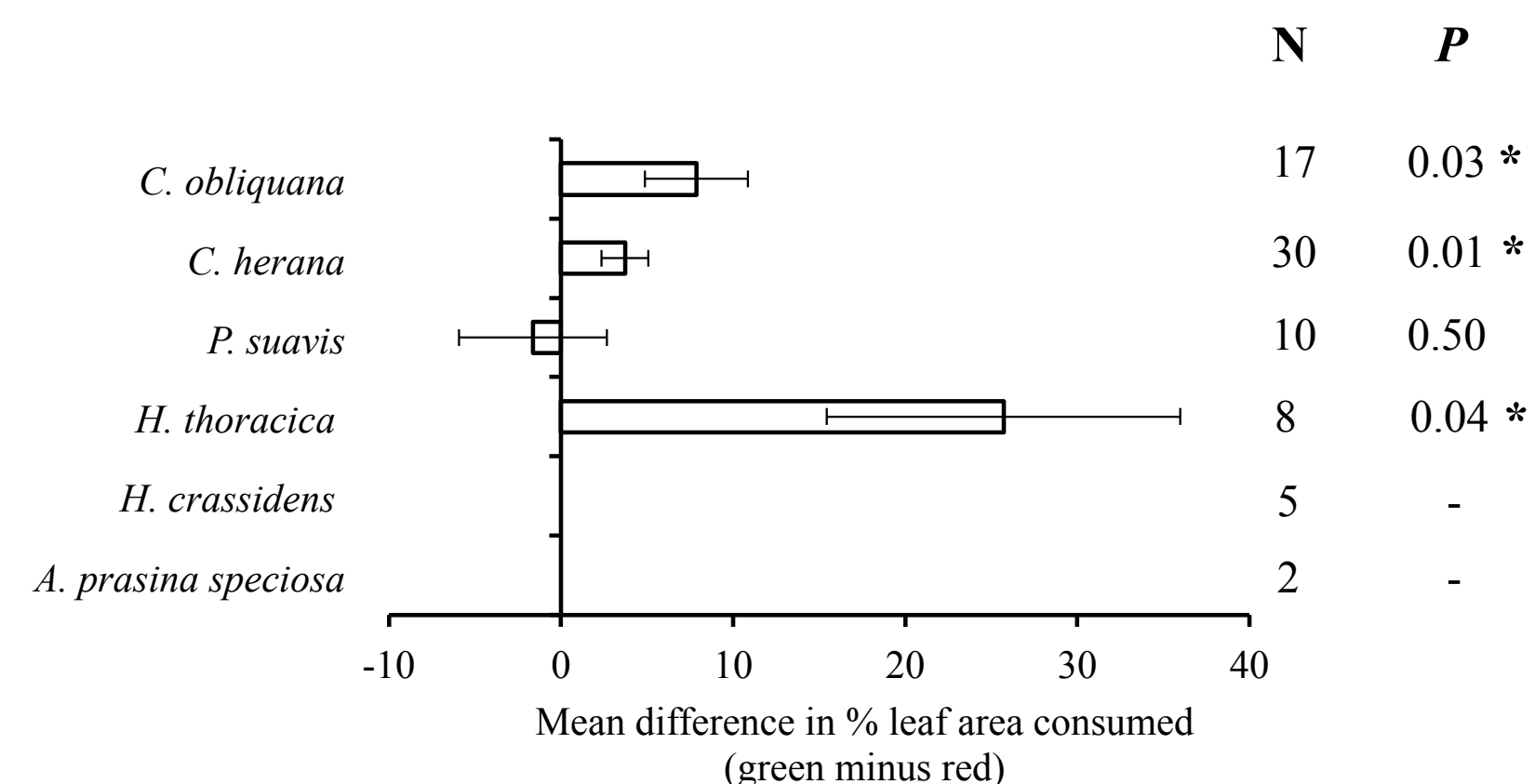

Fig. 5.2 Feeding preferences of a variety of endemic NZ chewing invertebrates for green and red coloured $P$. colorata leaves (Means $\pm \mathrm{SE}$ ). Bars to the right of the origin of the $\mathrm{x}$-axis indicate that insects preferentially consumed green leaves. Asterisks indicate statistical significance (Wilcoxon signed ranks test, $P<0.05$ ).

\subsubsection{Oviposition results}

C. obliquana females did not display an oviposition preference for either red or green $P$. colorata leaves $\left(\chi^{2}=4,2 \mathrm{df}, P=0.13\right)$. However, most replicates did not show any evidence of eggs upon either red or green leaves (Table 5.3). In many cases either the male or female moths did not successfully emerge from the pupae or became stuck upon drops of condensation, which precluded mating and oviposition.

Table 5.3 Frequency of oviposition by gravid C. obliquana females upon both green and red, either green or red, or neither P. colorata leaf in two types of bioassays (pots and petri).

\begin{tabular}{lcccc}
\hline Method & Green & Red & Both & Neither \\
\hline Pots & 8 & 2 & 2 & 47 \\
Petri & - & 1 & - & 19 \\
\hline
\end{tabular}




\subsection{DISCUSSION}

Through field surveys and bioassays, I have identified the most likely invertebrate herbivores to participate in coevolved leaf-signalling interactions with $P$. colorata. Feeding preference bioassays showed that brownheaded leafrollers (C. obliquana and C. herana) and Auckland tree weta (H. thoracica) discriminate between red and green $P$. colorata leaves. All three species preferentially consumed leaf material from green, rather than red leaves (Fig. 5.2). I was unable to detect any oviposition preference for green or red P. colorata leaves (Table 5.2) though the high mortality rates precluded definite conclusions. Results from these bioassays, combined with my field surveys suggest that Ctenopseustis spp. leafroller larvae are likely signalling partners for $P$. colorata.

In multiple trials, $H$. crassidens and $A$. prasina speciosa did not consume any $P$. colorata leaf material (Fig. 5.2). After three days in a chamber with P. colorata leaves, I placed some pōhutukawa foliage in with the stick insects; they started to feed upon the pōhutukawa before I had let go of it! Polygodial has a strong antifeedant effect on some species of lepidopteran, coleopteran and hemipteran (aphids) herbivores (Asakawa et al. 1988, Gerard et al. 1993, Powell et al. 1993, 1995). My results suggest that, irrespective of leaf colour, $P$. colorata's defences may effectively deter a broader taxonomic array of invertebrate herbivores.

Psuedocoremia spp. larvae (Lepidoptera : Geometridae [loopers]) have been reported to be highly abundant upon $P$. colorata in the field (Norton 1980), though they were rare at my field sites (Table 5.1). In my feeding preference bioassays, $P$. suavis consumed $P$. colorata but did not discriminate between red and green leaves. A lack of discrimination between differently defended hosts may indicate the herbivore is a specialist and has adapted to the defence compounds of their host (Bowers and Puttick 1988). However, P. suavis is well known to consume a wide range of NZ native and introduced plants (Berndt et al. 2004). Norton (1980) reported that a different species of Pseudocoremia, P. fasciculata, is a specialist herbivore, obligate upon P. colorata, although evidence to the contrary was presented by Stephens (2001). Regardless of the breadth of their diet, Psuedocoremia spp. larvae appear unaffected by $P$. colorata's defences, and therefore are unlikely to exert selective pressure to reinforce coevolved leaf signalling. 
Consistent with Barrett (1991), the NZ endemic Auckland tree weta, H. thoracica, did consume $P$. colorata. Moreover, H. thoracica discriminated between red and green leaves. However its distribution does not tightly match that of $P$. colorata. It is restricted to central and northern parts of the North Island with populations in fragmented pockets of bush as far south as Levin, and is absent from WellingtonWairarapa region (Trewick and Morgan-Richards 1995). The distribution of Wellington tree weta, $H$. crassidens, more closely matches that of $P$. colorata, being found throughout the central and lower North Island, as well as northern parts of the South Island (Trewick and Morgan-Richards 1995, Gibbs 2001). However $H$. crassidens did not consume $P$. colorata. Two species of South Island tree weta, $H$. ricta and $H$. femorata, have been found roosting in $P$. colorata trees (Townsend et al. 1997) but there are no records of them consuming $P$. colorata leaf material. Fossil and pollen records of Pseudowintera spp. have been found in several locations across both the South and North Islands of New Zealand (McGlone and Topping 1977, Pole 2007). However, the clear genetic separation between North Island and South Island Hemideina weta clades suggests $H$. crassidens dispersed to the South Island relatively recently, and that $H$. thoracica has long been confined to the North Island (Trewick and Morgan-Richards 1995, Morgan-Richards and Gibbs 2001). Therefore the historic distribution of $H$. thoracica argues against them being the primary selective force driving the coevolution of leaf colouration in P. colorata.

In contrast to the weta, Ctenopseustis spp. leafrollers exhibited a significant preference for green over red leaves. Hamilton and Brown (2001) discussed the ideal herbivore characteristics that will most likely result in a coevolved plant-herbivore signalling system. Below I discuss each quality in turn, focusing upon Ctenopseustis spp. leafrollers.

\subsection{1 a) Long history of association}

Pseudowintera colorata and Ctenopseustis spp. leafrollers are both endemic to NZ, and both plant and herbivore are thought to be of Gondwanan origin (Feild et al. 2002, Regier et al. 2012). Within NZ, both are distributed widely across both the North and South Islands (Allan 1961, Wearing 1999, Wayman et al. 2010) and cooccur in the wild (Norton 1980, Cooney et al. 2012, Youard 2012). Unlike deciduous 
tree species, the red colouration of $P$. colorata leaves is maintained year round. Similarly, larval and adult leafrollers can be found on host plants throughout the year, although there are peaks in population density in the late spring and autumn (Wearing 1999).

\section{5 .2 b) Mobility among host plant individuals}

The leaf signalling hypothesis requires that the herbivore has a life stage that is mobile enough to actively disperse among different host individuals of varying suitability. The minimum dispersal distance for different plant-herbivore associations varies, depending on the size and density of the host plants and the size and mobility of the insect. Similar to the winged aphids of the autumn leaf system, the most consistently abundant herbivore upon $P$. colorata has a flying life-stage. Adult moths of C. obliquana are able to fly at temperatures above $\sim 7^{\circ} \mathrm{C}$ (Wearing 1999) and are mobile enough to disperse among $P$. colorata in the field. In addition, some lepidopteran larvae, including representatives from Tortricidae, are mobile and can disperse through crawling, or descending on a silk thread, onto nearby morepreferable hosts (Norton 1980, Harris et al. 1995, Zaluki et al. 2002, Carroll et al. 2006). The ability of C. obliquana and C. herana larvae to use these mechanisms to disperse from shelter species to crop plants has been noted (Wearing 1999).

P. colorata hosted at least one species of scale insects at both Belmont and Otaki Forks. These organisms frequently settle within $<1 \mathrm{~m}$ from their mother (MagsigCastillo et al. 2012). Although travelling longer distances is possible through wind or phoretic dispersal via hitchhiking on other more mobile organisms (Magsig-Castillo et al. 2012), these processes are unlikely to provide scale insects with a means to actively select among host plants.

\section{5 .3 c) Damaging to the host plant}

P. colorata leaves commonly exhibit signs of feeding damage. Norton (1980) noted that, 'During my perusal of several hundred herbarium specimens of $P$. colorata, those with undamaged leaves were sufficiently rare for me to record the fact in my notebook' (p. 31). As stick insects, weta and lepidopteran larvae all possess chewing mandibles, reliable identification of which species of insect is responsible for 
herbivory is not easily possible, particularly for feeding around the margins of leaves. However, data from my field surveys and observations suggest that Ctenopseustis spp. leafrollers are likely to be responsible for the majority of leaf damage observed upon P. colorata at my field sites. Throughout three years of fieldwork, leafrollers were consistently highly abundant at my field sites. During my census of leafroller larvae at the Belmont trig site (see Chapter 2), each of the 60 plants I searched had leafroller larvae on them. The density of leafroller larvae upon one P. colorata plant was $130 \mathrm{~m}^{-2}$ canopy surface area (population median $=13 \mathrm{~m}^{-2}$, interquartile range $\left[\mathrm{IQR}=\mathrm{Q}_{1}\right.$ to $\left.\mathrm{Q}_{3}\right]=5$ to $30 \mathrm{~m}^{-2}$ ).

By consuming photosynthetic tissue, Ctenopseustis spp. leafrollers may have a measurable direct negative effect upon $P$. colorata (but see 'Tolerance vs. resistance to herbivores' section 2.5.3). Leafrollers may also have indirect impacts on the photosynthetic potential of $P$. colorata through leaf deformation (caused by herbivory of young shoots), by webbing leaves together to form shelters (reducing the leaf area exposed to direct sunlight; see Fig. 2.1E) and suppression of photosynthesis of remaining leaf tissue (Zangerl et al. 2002, Nabity et al. 2009). Leafroller feeding is also thought to increase a plant's susceptibility to virus and fungal infections (Wearing 1999). While I did not observe any inverse correlation between the number of leafrollers upon P. colorata and plant fitness (see Chapter 2) in the field, such a link may have been obscured by variation in photosynthetic performance among $P$. colorata individuals (see Chapter 4).

\section{5 .4 d) Discriminating (preferring the less defended/more suitable host trees)}

Redder P. colorata leaves are poor quality food compared to green leaves, as they tend to contain higher levels of sesquiterpene dialdehyde compounds (Youard 2012, plus see Chapter 2), known to be potent antifeedants (Asakawa et al. 1988, Gerard et al. 1993, Powell et al. 1993, 1995). My attempts to assess the fitness impacts of the higher concentrations of sesquiterpene dialdehydes found in red leaves on the growth and fecundity of C. obliquana were unsuccessful (Appendix B). However, experimental and correlative evidence corroborate to show that Ctenopseustis spp. leafrollers (being the most abundant chewing herbivore species to consume $P$. colorata leaves at my field sites) do discriminate among $P$. colorata leaves and 
individuals of varying suitability. Lab bioassays showed that Ctenopseustis spp. leafrollers do discriminate between leaves of differing quality, as they consumed on average $5 \%$ greater leaf area from green than red leaves (Fig. 5.2). In the field, redder leaves incurred a similarly small but statistically significant benefit in term of herbivory (Cooney 2012 and Chapter 2). Herbivory of Columnea consanguinea, another plant with variation in non-senescing leaf colour, showed a similar pattern but with much greater magnitude: in the field, leaves with red spots experienced on average $70 \%$ less area removed than leaves without red spots (Wong and Srivastava 2011). In the same study, feeding preference trials showed that katydids preferentially consumed non-anthocyanic leaf discs. However, unlike $P$. colorata, variation in defence or quality among red and green leaves of $C$. consanguinea has not been studied, therefore the mechanism of the katydid discrimination is unknown.

In the field, P. colorata trees with redder leaves hosted fewer Ctenopseustis spp. leafroller larvae than matched neighbouring green trees (Chapter 2). The non-random distribution of larvae in the field may be the result of larval preferences (dispersing to select more suitable host trees), oviposition preferences of gravid females (laying their eggs on most suitable host for the development of their young), or through other mechanisms independent of host preferences (i.e. increased larval mortality on red plants). While oviposition preferences of some Lepidoptera do match the intraspecific variation in host suitability (Gripenberg et al. 2010), neither Cooney et al. (2012) nor I detected any significant oviposition preference for green over red $P$. colorata leaves by C. obliquana adult females. Therefore, differences in herbivore abundance between red and green $P$. colorata trees may indeed be a product of larval dispersal preferences.

\section{5 .5 e) Visual stimuli used when discriminating among hosts}

For leaf colour to be a visual signal, the coevolved herbivore partner must use visual stimuli when selecting host plants. For the putative autumn leaf-aphid system this caused much contention, as aphid eyes lack a red receptor (Schaefer and Wilkinson 2004, Chittka and Döring 2007, Döring and Chittka 2007). Regardless, Döring et al. (2009) demonstrated that aphids perceive and respond to green and red colours. These colours are distinguishable, based on the relative ratio of the photon catches of 
aphids' green and blue photoreceptors. The spectral sensitivities of the stemmata of Ctenopseustis spp. larvae are unknown. Lepidopteran larvae, including one species within Tortricidae, the family containing C. obliquana, do respond to colour cues when dispersing and are attracted to green stimuli when walking and descending via silk threads (Harris et al., 1995; Singh \& Saxena, 2004). Cooney et al. (2012) assessed the role of vision in the leaf selection of $C$. obliquana larvae, by carrying out feeding preference trials under different coloured light conditions. In that study, colour cues were found necessary for discrimination between red and green $P$. colorata leaf margins by $C$. obliquana larvae.

\subsubsection{Other considerations}

It is recognised that plant species can participate in coevolution with multiple herbivores (Thompson 2005). The genus Ctenopseustis contains several species that, aside from sex pheromone differences, are not easy to distinguish morphologically (Dugdale 1990, Carraher et al. 2012). Incomplete lineage sorting makes DNA barcoding unreliable (Langhoff et al. 2009). Thus, I was unable to identify

Ctenopseustis leafrollers from the field to species level. However, feeding preference bioassays of two Ctenopseustis species sourced from lab populations indicated that discrimination between red and green $P$. colorata leaves may be a feature of the genus (unlike Hemideina spp.).

Some plant defence traits may have arisen through coevolution with animals that are extinct or no longer interacting with the plant (Janzen 1980). There has been much speculation on the selective pressures that browsing by extinct ratites (called moa) may have exerted on New Zealand's flora. Greenwood and Atkinson (1977) postulated that selection by moa might have driven evolution of chemical defences in P. colorata, and subsequent visual mimicry by Alseuosmia pusilla. However, to my knowledge, no Pseudowintera spp. leaf material or pollen have been identified in any moa coprolite records. 


\section{Nutrient status of red and green Pseudowintera colorata leaves}

\subsection{ABSTRACT}

The leaf signalling hypothesis proposes that red leaf coloration functions as a visual signal that conveys information about the defensive status of plants. However, there is a well-established link between nitrogen deficiency and leaf reddening. Additionally, leaf nutrients can influence foraging behaviour and performance of insect herbivores. Studies of leaf signalling rarely measure leaf nutrients and may therefore overlook alternative explanations for their results. $\mathrm{N}$ and $\mathrm{C}$ contents were measured for leaves from neighbouring matched pairs of red and green $P$. colorata. There were no significant differences in the amounts of, or ratio between, $\mathrm{N}$ and $\mathrm{C}$ between matched red and green leaves. This result indicates that differences in colour and herbivory among $P$. colorata leaves are not attributable to differences in leaf nutrients.

\subsection{INTRODUCTION}

The leaf signalling hypothesis proposes that red leaf coloration is a product of coevolution between plant and insect herbivores, and functions as a visual signal that conveys information about the defensive status of plants (Archetti 2000, Hamilton and Brown 2001). Plants with the brightest red leaf colouration are expected to have high leaf defences and host fewer insect herbivores, as approaching herbivores perceive, and are deterred by, the red signal (Archetti and Brown 2004). Much of the evidence presented in support of the leaf signalling hypothesis has taken the form of correlations between non-green leaf colour and reduced numbers of, or reduced amount of damage from, insect herbivores (Hagen et al. 2003, Archetti and Leather 2005, Karageorgou and Manetas 2006, Rolshausen and Schaefer 2007, Ramirez et al. 2008, Wong and Srivastava 2011, Cooney et al. 2012, Markwick et al. 2012). However, there are alternative explanations for differences in red leaf colour and herbivore abundance (or feeding damage) between individual plants. In order to test the leaf signalling hypothesis we must investigate other potential proximate mechanisms that make similar predictions. 
As a key building block for proteins, nucleic acids and photosynthetic pigments, nitrogen $(\mathrm{N})$ is vital for plant growth. In senescing autumn leaves, anthocyanins have also been proposed to act as photoprotectants, allowing for increased nutrient resorption from senescing leaves (Hoch et al. 2001). Several studies have found links between foliar anthocyanins and higher levels of $\mathrm{N}$ resorption (Hoch et al. 2003). Red colouration is also associated with nutrient deficiency in both senescing and nonsenescing leaves. Within-plant and among-species correlations between the timing of red coloration of senescing leaves and the degree of $\mathrm{N}$-limitation have been described (Schaberg et al. 2003, Sinkkonen 2008). However, for non-deciduous plants, this association has received much more study, due to the link between $\mathrm{N}$ and crop yields. Many plants experiencing $\mathrm{N}$ deficiency display up-regulated anthocyanin biosynthetic genes, and subsequently, reddening of their leaves (Chalker-Scott 1999, Close and Beadle 2003, Diaz et al. 2006, Peng et al. 2008). Previous studies of leaf signalling using non-autumn senescing species (Hughes et al. 2010b, Wong and Srivastava 2011, Cooney et al. 2012) have not assessed the nutrient status of leaves. Therefore, the observed inter-individual differences in leaf redness may be a result of differences in nutrition, not necessarily signalling.

The nutrient status of plants can also affect insect herbivore abundance and feeding damage. Nitrogen is vital for the growth of animals (Mattson 1980, Behmer 2009). Plant hosts with low levels of leaf $\mathrm{N}$ have been shown to incur reduced amounts of herbivory (Alonso and Herrera 2003) and lower numbers of herbivores due to the oviposition preferences of adults (Gripenberg et al. 2010) and through increased mortality/decreased performance of larvae developing upon N-poor diet (Zaluki et al. 2002, Gripenberg et al. 2010). Therefore, for hosts with non-senescing leaves, patterns of insect abundance consistent with leaf signalling might equally be explained by variation leaf $\mathrm{N}$.

Consistent with the predictions of the leaf signalling hypothesis, I found differences in the number of larval C. obliquana between matched pairs of red and green P. colorata individuals (Table 2.2). Additionally, redder P. colorata leaves sustained smaller amounts of herbivory relative to greener leaves (Fig 2.9). If there are differences in leaf nutrient content among red and green $P$. colorata trees then this may explain the observed non-random patterns in herbivore abundance and feeding damage. Here, in 
order to test the hypothesis that $P$. colorata red leaves are displaying nitrogen deficiency, I present data on the nitrogen and carbon content of leaves from neighbouring matched pairs of $P$. colorata plants of differing leaf colour.

\subsection{MATERIALS AND METHODS}

All leaves used in this experiment were taken from a subsample of the matched pairs of $P$. colorata trees at the Wellington site described in Chapter 2 of this thesis following the methods of Hagen et al. (2004). Each pair contained one tree whose leaves were predominantly green and one tree whose leaves were much redder. This design allowed me to assess the leaf nutrient variability between different coloured leaves whilst minimising differences in environmental conditions. I used the same 10 matched pairs of leaves as were used for pigment quantification in Chapter 4.

Leaf nutrient measurements were obtained with the help of Kaylyn Carpenter and Dr Niky Hughes (High Point University, North Carolina, U.S.) After excision of a $\sim 1$ $\mathrm{cm}^{2}$ section of leaf lamina from a point normal to the centre of the midrib, the remainder of the leaf was dried. Three pairs of leaves were dried at room temperature for 3 days before being ground in liquid $\mathrm{N}$. The remaining 7 pairs were ground in liquid $\mathrm{N}_{2}$ while fresh. Ground leaf tissue was freeze-dried for $24 \mathrm{~h}$, sealed in a microcentrifuge tube and mixed well by shaking. Ten mg dry-weight (DW) of each leaf was subjected to elemental analysis of $\% \mathrm{C}$ and $\% \mathrm{~N}$ content by the Environmental \& Agricultural Testing Service of the Department of Soil Science, North Carolina Sate University, USA.

The data were assessed for normality and homoscedacity using Shapiro-Wilk and Levene's tests. Following this, paired t-tests were used to look for differences in $\% \mathrm{~N}$, $\% \mathrm{C}$, and the ratio of $\mathrm{C}: \mathrm{N}$ between matched red and green P. colorata leaf tissue. All statistical analyses were carried out using PSAW/SPSS Version 18.0 (Chicago, IL, USA).

\subsection{RESULTS}



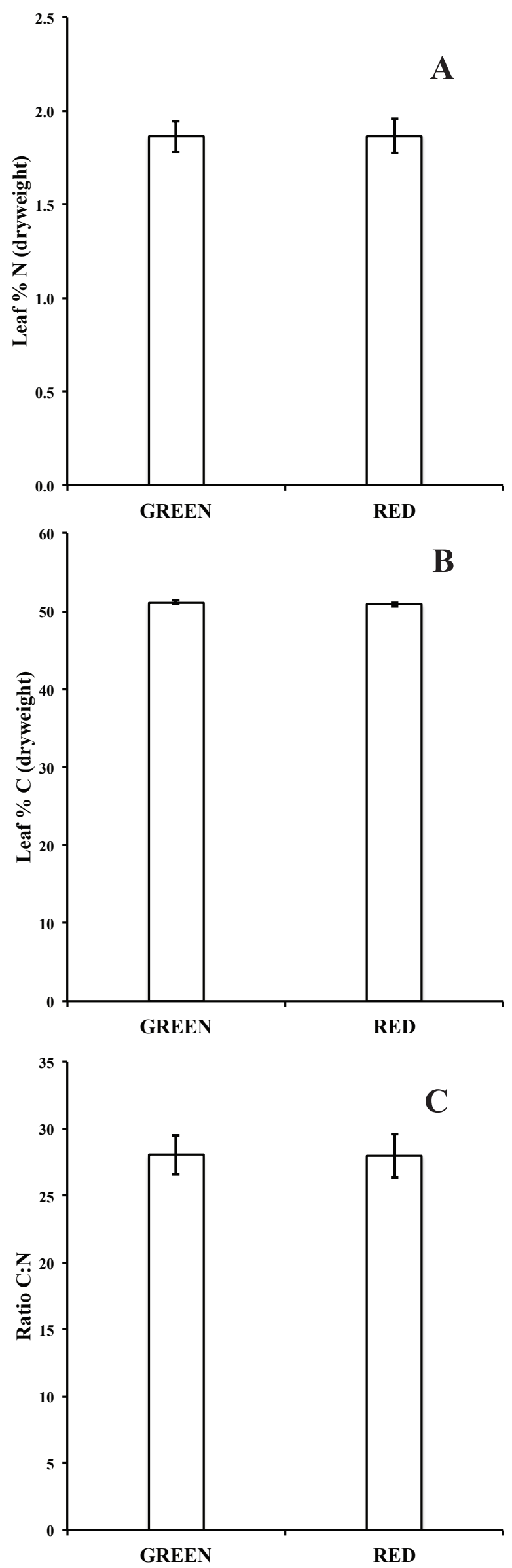

Fig. 6.1 Leaf nutrient content of 10 pairs of fully expanded green and red P. colorata leaves. Mean $( \pm$ SE) \% DW content of $(\mathbf{A}) \mathrm{N} ;(\mathbf{B}) \mathrm{C}$; and (C), ratio of C:N. 
Leaf $\mathrm{N}$ content ranged from 1.3 to $2.2 \% \mathrm{DW}$. Leaf $\mathrm{C}$ content ranged from 49.4 to $52.3 \%$ DW. No difference between red and green pairs in $\% \mathrm{~N}(\mathrm{t}=0.03, P=0.9$; Fig. 6.1A), \% C ( $\mathrm{t}=0.89, P=0.40$; Fig. 6.1B), or the ratio of $\mathrm{C}: \mathrm{N}(\mathrm{t}=0.02, P=0.98$;

Fig. 6.1C). There was no difference in the results when the three pairs of leaves that experienced a different drying regime (i.e. room dried, before being ground and freeze dried) were excluded.

\subsection{DISCUSSION}

In this study I found no evidence of a difference in the amounts of, or ratio between, $\mathrm{N}$ and $\mathrm{C}$ among red and green $P$. colorata leaves. This result indicates that the difference in leaf colour of $P$. colorata leaves is not due to difference in nutrient status. Additionally, although leaf nutrient data were not collected for the specific leaves for which feeding damage was recorded (in Chapters $2 \& 5$ ), this result suggests that differences in herbivory rates among differently coloured leaves (see Fig $2.9 \&$ Fig. 5.2) were not due to differences in the nutritional quality of those leaves. Rather, this result strengthens my assumption that differences in herbivory among red and green leaves reflects differences in chemical defences, specifically polygodial.

These leaf nutrient findings have implications for plant fitness. Due to its role in many essential metabolic processes, plant $\mathrm{N}$ status can affect plant growth and, after herbivory, compensatory regrowth (i.e. tolerance) (Rosenthal and Kotanen 1994). Leaf $\mathrm{N}$ values for both red and green plants were low, though within the typical range of leaf $\mathrm{N}$, indicating that these plants were not severely $\mathrm{N}$ deficient (Mattson 1980, White et al. 2000). As there were no statistical differences in leaf $\mathrm{N}$ between matched red and green plants, any inter-individual differences in leaf physiology or plant growth rates are not likely to be a result of leaf colour-specific nutrient differences. However, this study only included a narrow band of leaf age. It would be interesting to compare variability in leaf $\mathrm{N}$ among red and green leaves through out their ontogeny.

For future research, there is another quantitative method that would reveal more information about $P$. colorata leaf nutrient status and how this may mediate plantcaterpillar interactions. The Kjeldahl assay provides a measure of reduced nitrogen within leaf tissue i.e. amino acids and proteins (Ehleringer et al. 1986). Animals must 
sequester amino acids and proteins from their diet in order to grow (Mattson 1980). Thus the Kjedahl assay may give more information on the relative "quality" of leaf $\mathrm{N}$ from the herbivores' perspective.

Patterns in foraging by specific insect herbivores are likely to be the reflect both plant defences and plant nutrition (Simpson and Raubenheimer 2001). The influence in variation in the nutritional and allelochemical content of plant tissue on insect behaviour and physiology can be complex. Simpson and Raubenheimer (2001) found that, when fed a diet containing an ideal ratio of protein to carbohydrate, variation in the levels of tannic acid within the diet did not affect the growth or mortality of locusts. However, at progressively more nutritionally imbalanced diets, the influence of the defence levels on herbivore consumption rate, growth and mortality became more significant. While we do not know the optimal nutritive requirements of $C$. obliquana, the fact that there was no significant difference in leaf $\mathrm{N}$ : $\mathrm{C}$ ratio (proxy for protein to carbohydrate ratio) between red and green leaves suggests that variation in defences and/or leaf colour (i.e. signalling) must explain differences in herbivore abundance and feeding damage.

\subsection{CONCLUSIONS}

I did not detect any difference in the amount of, and ratio between, $\mathrm{N}$ and $\mathrm{C}$ among samples of red and green leaf tissue from matched pairs of $P$. colorata. This result suggests that variation in leaf colour among $P$. colorata individuals is not due to differences in plant nutritive status. Furthermore, it is likely that non-random patterns of herbivory among differently coloured $P$. colorata leaves are not due to nutrient differences, but rather differences in leaf allelochemical defences. 


\section{General discussion}

\subsection{INTRODUCTION}

Anthocyanins are synthesised in the leaves of many plants, however the adaptive significance of these pigments has been the subject of heated scientific debate for more than a century. It has been postulated that their red colours may function as visual signals through coevolution between herbivorous insects and their host tree species. However, the hypothesis lacks solid empirical evidence, as it has proved difficult to distinguish experimentally between leaf signalling and alternative, nonexclusive hypotheses. I exploited the natural polymorphism in leaf colour of $P$. colorata to test the predictions of this leaf signalling hypothesis for foliar anthocyanins, while controlling for, and, where possible, collecting data to assess the relative influence of alternative hypotheses.

The main results of the research contained in my thesis collectively support four important conclusions:

\subsection{There is strong empirical evidence in support of a leaf signalling function for foliar anthocyanins in Pseudowintera colorata.}

This thesis presents the strongest empirical evidence to date in support of leaf signalling. My data are consistent with the first and second predictions of the leaf signalling hypothesis, namely: 1) early autumn leaf colour change and 'bright' nongreen colouration is a signal of a tree's high defensive commitment, relative to conspecifics; and that, 2) upon perceiving the leaf colour signal, insect herbivores should alter their behaviour accordingly, selecting less well-defended host trees (see Chapter 1.6).

In two distinct populations of $P$. colorata, both leaf colour and foliar anthocyanin content correlated positively with the levels of the defence compound polygodial within leaves. Polygodial, a sesquiterpene dialdehyde, is the product of a distinct biochemical pathway to that of anthocyanin, and the mechanism by which levels of polygodial and anthocyanin co-depend is not clear. The Defence Indication hypothesis states that correlations between leaf colour and defence levels, and/or leaf colour and herbivory, can arise if the pigment and defence compounds are products of 
the same biochemical pathway (Schaefer and Rolshausen 2006). Pseudowintera colorata is the first plant species investigated thus far for which the Defence Indication hypothesis cannot be considered an alternative explanation for correlations between herbivory and leaf colour

Consistent with the second prediction of the leaf signalling hypothesis, redder $P$. colorata leaves incurred less feeding damage (and less variability in feeding damage) than greener leaves at the two populations investigated. Additionally, for the Wellington population, counts of larvae between red and green matched neighbouring P. colorata revealed that red trees hosted fewer caterpillars. In lab choice trials, two species of leaf roller caterpillars (Ctenopseustis spp.) consumed a greater area from green leaves than red leaves, as did a weta species. Moreover, Cooney et al. (2012) demonstrated that $C$. obliquana, a primary herbivore of $P$. colorata, uses colour cues to mediate its feeding decisions. In behavioural assays to assess the role of volatile cues, VOC profiles of damaged red and green $P$. colorata leaves did not affect orientation behaviour of $C$. obliquana larvae. These labs trials support my assumption that the observed non-random patterns of feeding damage in the field are at least partially attributable to visual-based insect preferences.

Most previous studies have investigated these two predictions in isolation, often using different study systems, their data unable to exclude the Defence Indication hypothesis. In contrast, all the data in this thesis were collected from the same study system, and some of it is replicated from two populations. My results, especially when considered in combination with those of Cooney et al. (2012), make a compelling case for a leaf signalling function for foliar anthocyanins in P. colorata.

\subsection{In terms of herbivory, the effect of leaf signalling in P. colorata is small.}

Although leaf signalling via foliar anthocyanins presented a statistically significant benefit to $P$. colorata in the season studied, the effect was small. At the Wellington population, using a matched pair design, redder $P$. colorata trees hosted on average five fewer leaf roller caterpillars than did neighbouring matched green trees (see Chapter 2); although this difference was statistically significant, caterpillars were still abundant upon both red and green trees. 
The majority of studies on leaf signalling have not considered the magnitude of possible leaf signalling benefits, and have instead focused upon simply whether their data support or contradict leaf signalling predictions. However the two studies that did, found that variability in non-green autumn leaf colour explained more than $80 \%$ of the variability in the numbers of herbivores (Archetti and Leather 2005) and more than $25 \%$ of the variation in the amounts of insect damage (Hagen et al. 2003). Alternative explanations cannot be ruled out in these studies, and direct comparisons with my study are complicated by methodological dissimilarities, however the difference between these studies and mine suggests that the efficacy of signalling may be lower in non-senescing leaf systems.

A small effect of signalling is, perhaps, not surprising given that many factors influence insect host selection and subsequent feeding behaviour (Schoonhoven et al. 2005); indeed, many factors influence foliar anthocyanin levels (Chalker-Scott 1999), and anthocyanin pigments apparently have multiple functions within leaves (Gould 2004). My results suggest that in non-senescing leaves, signalling is probably only a small component of a diverse suite of plant protection mechanisms.

\subsection{Alternative hypotheses cannot be ignored.}

It has been repeatedly pointed out in the leaf signalling literature, that there are other possible explanations for patterns of leaf chemistry, colour and herbivory which are consistent with the first and second predictions of the leaf signalling hypothesis (Wilkinson et al. 2002, Schaefer and Wilkinson 2004, Schaefer and Rolshausen 2006, Rolshausen and Schaefer 2007). However, few studies have directly investigated these alternative hypotheses. This is possibly due to the insistence by some authors that research should focus upon finding the adaptive reason for foliar anthocyanins, rather than consider multiple functions (Schaefer and Gould 2007, Archetti et al. 2009).

I was fortunate that my study species circumvented the Defence Indication hypothesis (see above). However, I also investigated other alternative hypotheses. In Chapter 2.5, I proposed that variation in leaf nutrient levels may be a proximate explanation for variation in leaf colour, and that this might also be expected to cause colour- 
correlated variation in herbivory levels. Leaf nutrient assays allowed me to rule out this explanation for leaf colour variation in P. colorata.

It has been hypothesised the VOC profiles emitted by leaves vary with leaf colour, and that these volatile cues may influence host selection and tritrophic interactions (Holopainen 2008, Yamazaki 2008a, b, Holopainen et al. 2010). Such mechanisms may provide an alternative explanation for leaf colour correlated variation in herbivore abundance and/or feeding damage. For example, leaf-colour specific VOC differences may be used by adult herbivore life stages or predator/parasitoid species as a cue, which could result in differences in oviposition or predation/parasitism between different coloured $P$. colorata in the field (Turlings et al. 1990, Kessler and Baldwin 2001, Allison and Hare 2009, Hare 2011). I compared VOC from leaves from red and green matched pairs of $P$. colorata. The VOC profiles released from intact, herbivore-, and mechanically-damaged red and green $P$. colorata leaves did not reliably indicate leaf colour. In behavioural assays, the VOC profiles did not affect larval herbivore behaviour. I conclude that volatiles are not cues for leaf colour in P. colorata, and can therefore rule out this alternative explanation.

Given the abundant evidence that anthocyanin pigments can play photoprotective roles in plant tissues (Table 4.4), Lev-Yadun and Gould (2007) hypothesized that anthocyanins in leaves may function for both photoprotection and signalling. Two other empirical studies have simultaneously investigated leaf signalling and physiology (Karageorgou and Manetas 2006, Rolshausen and Schaefer 2007), both of which found indirect evidence consistent with both leaf signalling and photoprotection. I investigated the influence of anthocyanins upon photosynthesis of $P$. colorata leaves, but did not find evidence that foliar anthocyanins provide a photoprotective advantage; rather, I found that redder leaves had the lower light saturated $\mathrm{CO}_{2}$ assimilation rate. Thus, the influence of anthocyanin pigments upon photosynthesis is not always predictable (Table 4.4), despite the fact that light absorption is an 'immutable property of coloured anthocyanins' (Hatier and Gould 2008a), and it cannot, therefore, be disregarded in discussions of leaf signalling. 


\subsection{Inter-individual variation in non-senescing leaf colour may be stable due to a trade off between signalling and photosynthesis.}

I exploited the natural polymorphism in leaf colour in one population of $P$. colorata to investigate a third prediction of leaf signalling, that signalling is adaptive and therefore confers a fitness benefit. Contrary to this prediction, matched pairs of red and green $P$. colorata trees had similar mean fitness, as measured by reproductive effort. This result suggests that, in this population, for the season studied, the interindividual leaf colour variation may have been evolutionary stable. It is important to note, however, that the data presented in this thesis are not sufficient to detect natural selection, as is required to test evolutionary stability. In the discussion of Chapter 2 I proposed four explanations to explain why a balanced polymorphism in leaf colour may occur, by synthesising ideas from the anthocyanin and leaf signalling literature (reviewed in Chapter 1).

One of my ideas was that signalling is likely to incur energetic costs for the production, transportation and maintenance of pigments and defence compounds, as well as impose costs to photosynthesis from the presence of light absorbing coloured pigments in non-senescing leaves. As herbivore levels vary throughout and among years then the fitness payoffs from plant defence are likely to vary too (Simms 1992, Hare and Elle 2004). If the levels of herbivore pressure are low then the costs of investing and maintaining a signal may result in equal fitness of signalling and nonsignalling plants. In the absence of herbivores altogether, one might expect signalling plants to be at a disadvantage. Therefore, in this scenario, interindividual variation in leaf colour would be maintained in the population by balancing selection (Levene 1953, Kawagoe et al. 2011).

My investigations into the photosynthesis of leaves from matched red and green individuals revealed that there is potentially a trade off involved in producing a visual signal via foliar anthocyanin pigments. Redder leaved $P$. colorata individuals hosted slightly fewer herbivorous lepidopteran larvae (Table 2.2), however leaves from these individuals displayed a lower light saturated $\mathrm{CO}_{2}$ assimilation rate than leaves from greener individuals (Fig. 4.3). My observed equal fitness result suggests that, for the season studied, the benefits of signalling did not exceed the photosynthetic cost of maintaining pigments within $P$. colorata leaves. 
Most theories regarding the evolution of plant defences assume some trade off exists between growth and defence (Herms and Mattson 1992, Stamp 2003). As a consequence, the study of the costs of plant defence has attracted vast amount of study (Koricheva 2002, Strauss et al. 2002, Stowe and Marquis 2011). Leaf signalling is not a traditional resistance mechanism: it is not solely the presence of defensive compounds, but rather signalling (a coevolved interaction) that deters herbivores. Similarly, the potential costs to signalling hypothesised in this thesis are unusual. The cost from signalling is primarily to do with the effect of foliar anthocyanin pigments upon photosynthesis, rather than the metabolic costs of synthesising defence compounds, or subsequent indirect or ecological costs. Therefore, rather than a allocation cost, this could be considered an opportunity cost in terms of future potential resource acquisition (Strauss et al. 2002).

Intrinsic to the concept of a trade-off is the assumption that the resource is limiting; if a resource is not limiting, then an increase in its availability would not be expected to influence an organism's fitness (Mole 1994). I found differences in the light saturated $\mathrm{CO}_{2}$ assimilation rate of leaves from red and green plants. Referring to this difference as a 'cost of defence' assumes that carbon is limiting and, consequently, that the differences in assimilation do constrain plant fitness. Very little is known about the link between differences in carbon availability and plant growth and fitness (Smith and Stitt 2007). Further experimental work is needed to assess if differences in photosynthetic potential caused by foliar anthocyanins do indeed constrain fitness. If costs to signalling cannot be detected, then it is possible that the mechanism behind the maintenance of non-senescing leaf colour variation in $P$. colorata is not balancing selection through temporally variable fitness payoffs.

Other mechanisms have been proposed to explain the maintenance of phenotypic and genotypic variation within populations. Balanced polymorphisms can result from frequency-dependant selection, heterozygote advantage or, if the trait is not subject to selection, be maintained within a population by neutral genetic drift (Guzmán et al. 2011, Kawagoe et al. 2011). Alternatively, as discussed in Chapter 2, other processes may contribute to the equal fitness result between red and green trees: a leaf colour polymorphism may appear balanced if the fitness effects of signalling are obscured by selection acting upon other colour-correlated traits (e.g. VOC and tolerance). As 
discussed above, I found no differences in the VOC profiles of leaves from red and green $P$. colorata individuals. Damaged red and green leaves are unlikely to be differentially attractive to parasitoids or predators and, therefore, VOC-mediated interindividual differences in a plant's herbivore or pollinator community, and subsequent differences in plant fitness, are not likely to occur. Additionally, subsequent to the evolution of a signalling system, higher herbivory upon green trees may promote evolution of tolerance in green morphs (Strauss and Agrawal 1999, Fornoni 2011, Hakes and Cronin 2011). While I did not directly collect data regarding tolerance mechanisms, there may be some indirect evidence: in Chapter 4, data on the ratios of chlorophylls $a$ and $b$ within red and green leaves did not point towards leaf shading by foliar anthocyanins, instead perhaps the higher light saturated $\mathrm{CO}_{2}$ assimilation rate of green leaves is an adaptation to increase tolerance to herbivores (Strauss and Agrawal 1999).

I can rule out one of the potential explanations for the stability of leaf colour variation proposed in Chapter 2. I posited that foliar anthocyanins might help nutrient deficient plants through both signalling and photoprotection. However, I found no difference in the nutrient status of leaves from red and green individuals. Additionally, I found no difference in the sensitivity of red and green leaves to photoinhibition, and therefore no evidence for a photoprotective function for anthocyanin pigments within $P$. colorata leaves. While I was able to rule out this process for P. colorata, it is important to include leaf nutrient measurements in future studies of leaf signalling, due to the links between leaf nutrients with leaf colour, photosynthesis, growth and herbivory (Mattson 1980, Rosenthal and Kotanen 1994, Chalker-Scott 1999).

Is a similar trade off likely to apply to other cases of interindividual leaf colour variation? Yes - certainly for non-senescing leaves. However, there may be no opportunity cost of signalling in autumn senescing leaves, as foliar pigments are not likely to impose a photosynthetic cost, but rather are expected to benefit energy balance of trees through nutrient resorption (Hoch et al. 2001, Hoch et al. 2003). For foliar anthocyanins in juvenile leaves, an a priori prediction of photosynthetic cost is difficult due to variation in leaf development strategies among species (Coley et al. 2005). However, the influence of pigments upon photoprotection and light screening in young leaves is likely to depend on the pattern of pigmentation. Irregular patches of 
anthocyanin pigments (as in this study) might be less likely to bestow a photoprotective advantage than pigments uniformly distributed over the leaf lamina.

Additional to the natural variation in leaf colour, anthocyanin pigmentation can be induced in P. colorata. After mechanical damage (including herbivory), small red lesions appear around damaged leaf tissue (Gould et al. 2002a). The function of this induced redness is unknown, although it has been hypothesised to mitigate the effects of abiotic stressors, or to protect against subsequent attack from pathogens and/or herbivores (Gould et al. 2002a). I was unable to detect co-induced biosynthesis of polygodial (data not shown), however the experiment was plagued by methodological difficulties. If there were no co-induced increase in defences, then induced pigmentation would weaken the correlation between leaf colour and defence, which would be expected to reduce the likelihood of the coevolution of leaf signalling (Rolshausen and Schaefer 2007). Further investigations into induced redness in $P$. colorata were not possible within the time constraints of this thesis. Most importantly, induced redness was not responsible for the matched pair inter-individual leaf colour variation in $P$. colorata.

Colour polymorphisms are ecologically relevant and of great interest for evolutionary biology (Forsman et al. 2008, McKinnon and Pierotti 2010, Wennersten and Forsman 2012). To date, studies investigating the mechanisms and implications of colour polymorphisms in plants have solely focused upon reproductive organs (most frequently flowers) not vegetative organs as in this study. Leaf signalling, as an hypothesised explanation for variation in both leaf colour and defence, has the potential to add important contributions to plant defence theory and evolutionary biology.

In light of my results, can the concepts from leaf signalling be applied to agriculture for crop protection? The answer depends what plant organ is harvested. If one is collecting reproductive tissues and the signalling mechanisms are similar to $P$. colorata (i.e. trade off between signalling and photosynthesis), then red and green morphs are likely to have no significant difference in yield. However, if one is collecting vegetative tissues (e.g. lettuce and cabbage), then reduced herbivory damage of red morphs would be desirable regardless of the fitness of individuals, 
especially for annual plants. Some horticultural studies show that red varieties of lettuce and cabbage are less attractive to butterflies, aphids and thrips than green varieties (Müller 1964, Griffiths and Shelton 2004, Jankowska 2006). However, it is important to note that in my study, signalling had only a weak effect and no individuals escaped herbivory entirely.

\subsection{CONCLUSIONS}

Taken together, my results suggest that foliar anthocyanins in $P$. colorata do function as visual signals, however their effect on herbivory is small. Inter-individual variation in non-senescing leaf colour in P. colorata may be stable due to a trade off between signalling and photosynthesis. My findings imply that the adaptive function of leaf colouration is more complex than the decade old debate on this topic acknowledges. Traditionally, studies testing red leaf hypotheses have presented their results as supporting either signalling $O R$ physiological hypotheses. My study shows that this approach is no longer applicable; without incorporating alternative hypotheses (in particular: the physiological consequences of non-green leaf pigments) it is not possible to reject leaf signalling. The potential for equal fitness pay offs has not previously received attention in leaf signalling literature. I identified and discussed four nonexclusive scenarios that highlight internal and external factors that may explain this polymorphism. Rather than hunting for evidence of leaf colour-correlated herbivory and chemistry patterns, the leaf signalling hypothesis will only be advanced by disentangling the various physiological and ecological differences between differently coloured individuals, and experimentally assessing the influence of these differences upon plant fitness. Discussions of leaf signalling need to follow the examples of other fields studying the interactions between plants and insects and move from overly simple models to those that incorporate more of the complexity that is observed in the natural world. 


\section{Appendicies}

\section{A. Induced vs. natural leaf redness}

Leaf signalling predicts a correlation between leaf redness and feeding damage. $P$. colorata leaves are naturally variable in colouration, but mechanical injury and insect feeding can induce the biosynthesis of additional anthocyanin pigmentation in leaf tissue immediately adjacent to damage (Gould et al. 2002a). When assessing the patterns of colouration and herbivory of $P$. colorata leaves from the field (see Chapter 2), some of the red area was natural redness (not obviously associated with any leaf damage) and some was induced redness after damage.

I closely inspected the digital image of each leaf and noted all areas of damage (identified by missing leaf area, or necrotic leaf tissue). Next, I estimated the proportion of red leaf area that was associated with this damaged tissue (typically a red margin around missing leaf area). Subsequently, I assigned each leaf to a category $(1-5)$ according to how much of the observed red area was associated with mechanical damage (Table A). I divided this category score by 5 to get a decimal, which I then multiplied by the red area measurement for that leaf. This adjusted the percent red leaf area measurement, reducing it according to how much of the red leaf area was induced.

To illustrate:

Low proportion of leaf redness associated with damage: score of $5=>5 / 5=1$

Leaf area measurement will be unchanged

High proportion of leaf redness associated with damage: score of $1=>1 / 5=0.2$ Leaf red area measurement for that leaf will be much reduced after being adjusted. 
Table A. Induced leaf redness classification categories

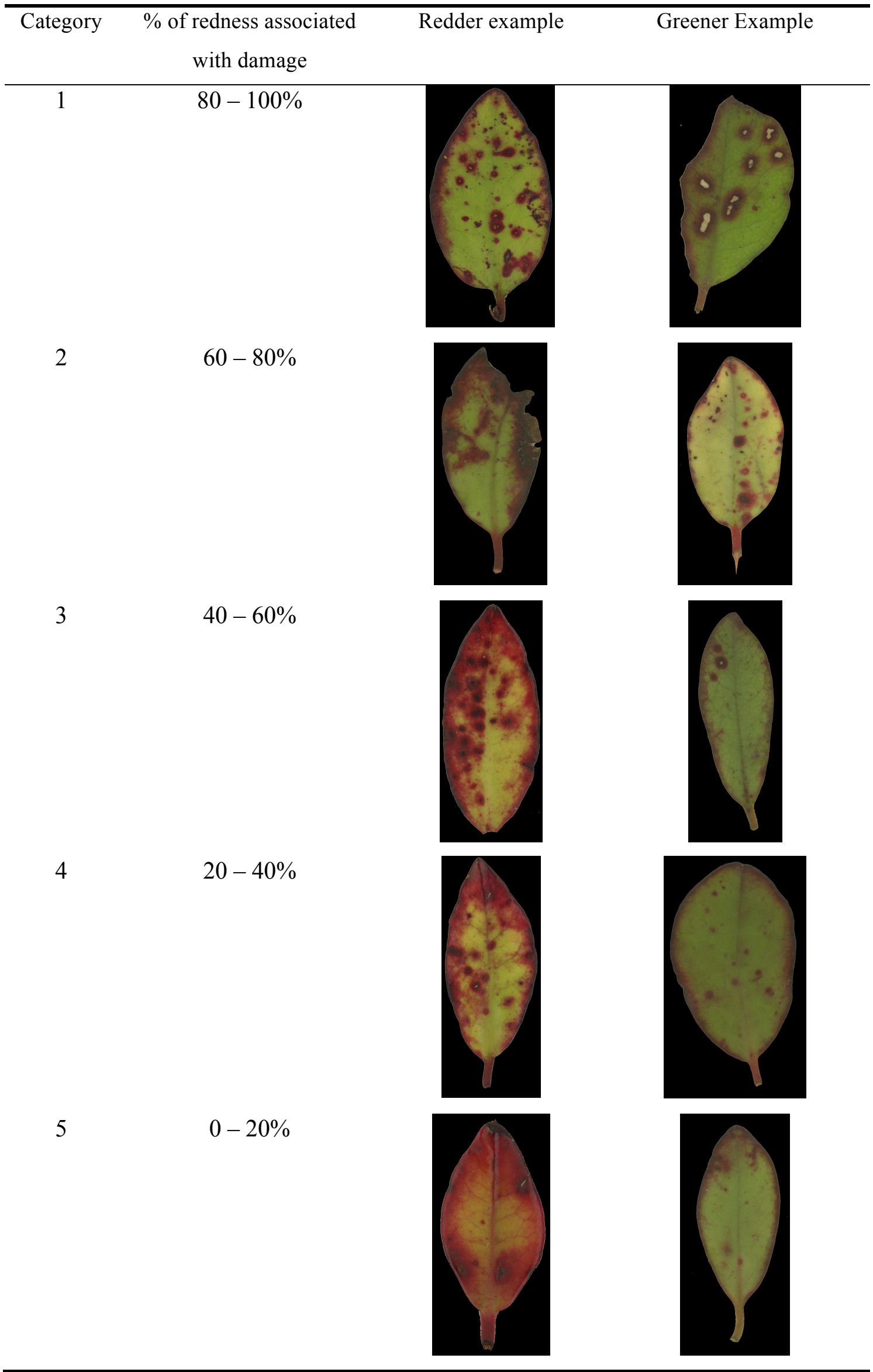




\section{B. Insect fitness upon diet of red or green P. colorata leaves}

\section{INTRODUCTION}

The third prediction of leaf signalling hypothesis states that, on average, signalling increases the fitness of participating plant and herbivore individuals. Only one study to date has attempted to assess fitness consequences of signalling for insect herbivores. Ramirez et al. (2008) used cage clips to measure aphid reproduction rates in spring upon trees that displayed different leaf colouration the previous autumn. No correlation was detected between host autumn leaf colour and aphid reproductive rate the following spring.

I tried two methods to assess whether a restricted diet of either red or green $P$. colorata leaves causes differences in the growth, development and fecundity of the brownheaded leafroller $C$. obliquana. Following similar methods to Barrington et al. (1993), I reared larvae on a diet of exclusively red or green $P$. colorata leaves, and planned to measure time-to-pupation and pupal weight. I also planned to mate pairs from within treatments, to count the number of resulting eggs.

\section{MATERIALS AND METHODS}

The first method was carried out between September and October 2011. Sixty 3rd instar C. obliquana larvae were reared in $5 \mathrm{~mL}$ test tubes with either two red or two green $P$. colorata leaves and a cottonwool bung. The leaves were all collected from the one matched pair of $P$. colorata trees from Belmont trig. Care was taken to ensure the leaves were all of a similar size and age. Leaves bearing insects, or exhibiting signs of herbivory were rejected. Test tubes were kept in incubator at $20^{\circ} \mathrm{C}, \sim 60 \%$ relative humidity and a 16:8 h (light:dark) photoperiod. To reduce leaf desiccation and maintain humidity within the tubes, a drop of distilled water was added twice a week. Fresh leaf material was collected weekly, and the old leaves were replaced.

The second insect fitness experiment took place between March and May 2012. Due to the difficulties involved with maintaining individual larvae within test tubes, I used twenty $5 \mathrm{~L}$ transparent plastic containers, with nylon mesh inserts within their lids. 
Ten matched pairs of $P$. colorata with the greatest contrast in leaf colour were selected. The terminal portion ( $\sim 20 \mathrm{~cm}$, bearing 20 to 30 leaves) of one stem from each tree was excised, recut under water and the base of the stem immersed in a florists tube. I thoroughly searched each stem and, after removing any insects, placed them in the containers. Using a paintbrush, I introduced seventeen one-day-old hatchling C. obliquana larvae on to leaves within each container. The containers were kept in a controlled temperature growth-room at $20^{\circ} \mathrm{C}$. A fluorescent light bank and rotating fan were connected to an automatic timer to maintain a 16:8 h (light:dark) photoperiod and to regularly circulate the air. Every week, fresh stems were collected from the same pairs of $P$. colorata, and placed in the appropriate container. The previous stems were kept in the containers for one week to allow the larvae sufficient time to migrate to the fresh leaf material. I did a thorough search before removing any leaf material from the containers and any remaining larvae were manually transferred to the fresh leaves. To reduce the influence of any variation in lighting, heating and airflow, the containers were rotated within the growth room weekly.

\section{RESULTS}

My attempts to rear C. obliquana larvae on a diet of either red or green $P$. colorata leaves were unsuccessful. Of the $3 \mathrm{rd}$ instar larvae reared within $5 \mathrm{~mL}$ test tubes, a large proportion did not survive to pupation (presumably succumbing to fungal attack). The remaining larvae were so few and displayed such pronounced variation in size within treatments that I abandoned the experiment. The growth rates of larvae reared from eggs in $5 \mathrm{~L}$ chambers were extremely low. When I concluded the experiment, due to time constraints, the largest observable larvae were only $2-4 \mathrm{~mm}$ long, and as such were clearly very far from pupation.

\section{DISCUSSION}

I was unable to assess the insect fitness implications of eating differently coloured leaves. Both the $5 \mathrm{~mL}$ test tube and $5 \mathrm{~L}$ box methods were unsuccessful within available time. I had also planned to mate pairs from within treatments, to count the number of resulting eggs, however no larvae pupated during these experiments, therefore this was not possible. 
Using fresh leaf material presented experimental difficulties. Moisture is vital to retain leaf tugur and freshness. However, too much humidity can promote fungal infection in captive insect larvae (G. W. Gibbs pers. comm.). The microclimate within the small containers, even though they were kept in a humidity-controlled incubator, must have been too damp.

Given the extremely low growth rates, the frequent and exhausting trips to the field site to gather fresh leaf material, plus thesis and family constraints, I abandoned the large container experiment after nearly twice the usual duration for pupation had elapsed (Barrington et al. 1993). However, Barrington et al. (1993) reared insects upon artificial diet (Singh 1983) with dried, finely ground leaf material mixed in. I chose not to do this as I thought that the highly reactive defence compounds in $P$. colorata would either complex with the artificial diet or be oxidised. Regardless, the long development time indicates that $P$. colorata is a poor diet to grow on. I later heard that, while $C$. obliquana can complete 4 - 6 generations per year on exotic orchard crops (http://www.hortnet.co.nz/publications/hortfacts/hf401027.htm, accessed May 2012), leafroller life cycle can take up to 12 months upon Pseudowintera spp. in the wild (G. W. Gibbs pers. comm.).

Future experiments testing the stability of polygodial and other $P$. colorata leaf extracts within an artificial diet matrix, and subsequent feeding trials, would be very informative. Experimental proof that variation in polygodial concentration (at a magnitude resembling that found between green and red $P$. colorata leaves) does cause fitness differences in C. obliquana would be strong evidence in support of a leaf signalling function for foliar anthocyanins in P. colorata. 


\section{References}

Adams III, W. W., C. R. Zarter, K. E. Mueh, V. Amiard, and B. Demmig-Adams. 2006. Energy dissipation and photoinhibition: a continuum of photoprotection. Pages 49-64 in B. Demmig-Adams, W. W. Adams III, and A. K. Mattoo, editors. Photoprotection, photoinhibition, gene regulation, and environment. Advances in photosynthesis and respiration. Springer, Dordrecht, The Netherlands. Vol. 21

Adir, N., H. Zer, S. Shochat, and I. Ohad. 2003. Photoinhibition - a historical perspective. Photosynthesis Research 76:343-370.

Aharoni, A., A. P. Giri, S. Deuerlein, F. Griepink, W. J. de Kogel, F. W. A. Verstappen, H. A. Verhoeven, M. A. Jongsma, W. Schwab, and H. J. Bouwmeester. 2003. Terpenoid metabolism in wild-type and transgenic Arabidopsis plants. Plant Cell 15:2866-2884.

Allan, H. H. 1961. Flora of New Zealand. Volume I. R. E. Owen, Government Printer, Wellington.

Allen, J. A. and R. Knill. 1991. Do grazers leave mottled leaves in the shade? Trends in Ecology \& Evolution 6:109-110.

Allison, J. D. and J. D. Hare. 2009. Learned and naive natural enemy responses and the interpretation of volatile organic compounds as cues or signals. New Phytologist 184:768-782.

Allmann, S. and I. T. Baldwin. 2010. Insects betray themselves in nature to predators by rapid isomerization of green leaf volatiles. Science 329:1075-1078.

Alonso, C. and C. M. Herrera. 2003. Developmental and spatial covariation of nutrients in growing leaves of Daphne laureola and their relationships with herbivory. New Phytologist 159:645-656.

Alves, P. L. d. C. A., A. C. N. Magalhães, and P. R. Barja. 2002. The phenomenon of photoinhibition of photosynthesis and its importance in reforestation. The Botanical Review 68:193-208.

Andersen, O. M. and M. Jordheim. 2005. The Anthocyanins.in O. M. Andersen and K. R. Markham, editors. Flavonoids: chemistry, biochemistry, and applications. CRC Press, Boca Raton, FL.

Andersen, O. M. and M. Jordheim. 2010. Anthocyanins. Encyclopedia of Life Sciences (ELS). John Wiley \& Sons, Chichester.

Archetti, M. 2000. The origin of autumn colours by coevolution. Journal of Theoretical Biology 205:625-630.

Archetti, M. 2007. Autumn colours and the nutrient retranslocation hypothesis: A theoretical assessment. Journal of Theoretical Biology 244:714-721.

Archetti, M. 2009a. Classification of hypotheses on the evolution of autumn colours. Oikos 118:328-333.

Archetti, M. 2009b. Evidence from the domestication of apple for the maintenance of autumn colours by coevolution. Proceedings of the Royal Society of London Series B-Biological Sciences 276:2575-2580.

Archetti, M. 2009c. Phylogenetic analysis reveals a scattered distribution of autumn colours. Annals of Botany 103:703-713.

Archetti, M. and S. P. Brown. 2004. The coevolution theory of autumn colours. Proceedings of the Royal Society of London Series B-Biological Sciences 271:1219-1223. 
Archetti, M. and S. P. Brown. 2006. Putting 'red alerts' in an ecological and evolutionary context. Bioessays 28:959-959.

Archetti, M., T. F. Doring, S. B. Hagen, N. M. Hughes, S. R. Leather, D. W. Lee, S. Lev-Yadun, Y. Manetas, H. J. Ougham, P. G. Schaberg, and H. Thomas. 2009. Unravelling the evolution of autumn colours: an interdisciplinary approach. Trends in Ecology \& Evolution 24:166-173.

Archetti, M. and S. R. Leather. 2005. A test of the coevolution theory of autumn colours: colour preference of Rhopalosiphum padi on Prunus padus. Oikos 110:339-343.

Asakawa, Y., G. W. Dawson, D. C. Griffiths, J. Y. Lallemand, S. V. Ley, K. Mori, A. Mudd, M. Pezechkleclaire, J. A. Pickett, H. Watanabe, C. M. Woodcock, and Z. N. Zhang. 1988. Activity of drimane antifeedants and related-compounds against aphids, and comparative biological effects and chemical-reactivity of (-)-polygodial and (+)-polygodial. Journal of Chemical Ecology 14:18451855.

Atkinson, N. 2001. Out on a limb, or a new branch of signalling theory? Trends in Ecology \& Evolution 16:603-603.

Barrett, P. 1991. Keeping wetas in captivity. A series of nine article for schools and nature lovers. Wellington Zoological Gardens, Wellington, New Zealand.

Barrington, A. M., G. B. Russell, and G. A. Lane. 1993. Effect of selected plants on the growth and development of leafrollers.in Proceedings of the 46th N.Z. Plant Protection Conference. The New Zealand Plant Protection Society Inc., Christchurch, N.Z.

Behmer, S. T. 2009. Insect herbivore nutrient regulation. Annual Review of Entomology 54:165-187.

Ben Zvi, M., Moyal, E. Shklarman, T. Masci, H. Kalev, T. Debener, S. Shafir, M. Ovadis, and A. Vainstein. 2012. PAP1 transcription factor enhances production of phenylpropanoid and terpenoid scent compounds in rose flowers. New Phytologist 195:335-345.

Bernasconi, M. L., T. C. J. Turlings, L. Ambrosetti, P. Bassetti, and S. Dorn. 1998. Herbivore-induced emissions of maize volatiles repel the corn leaf aphid, Rhopalosiphum maidis. Entomologia Experimentalis Et Applicata 87:133-142.

Berndt, L., E. G. Brockerhoff, H. Jactel, T. Weis, and J. Beaton. 2004. Biology and rearing of Pseudocoremia suavis, an endemic looper (Lepidoptera: Geometridae) with a history of outbreaks on exotic conifers. New Zealand Entomologist 27:73-82.

Blackiston, D. J., E. S. Casey, and M. R. Weiss. 2008. Retention of memory through metamorphis: can a moth remember what it learned as a caterpillar? PLoS ONE 3:e1736.

Blair, R. C. and J. J. Higgins. 1985. Comparison of the power of the paired samples $t$ test to that of Wilcoxon's signed-ranks test under various population shapes. Psychological Bulletin 97:119-128.

Boardman, N. K. 1977. Comparative photosynthesis of sun and shade plants. Annual Review of Plant Physiology 28:355-377.

Bowers, M. D. and G. M. Puttick. 1988. Response of generalist and specialist insects to qualitative allelochemical variation. Journal of Chemical Ecology 14:319334.

Bruce, T. J. A. and J. A. Pickett. 2011. Perception of plant volatile blends by herbivorous insects - finding the right mix. Phytochemistry 72:1605-1611. 
Bruce, T. J. A., L. J. Wadhams, and C. M. Woodcock. 2005. Insect host location: a volatile situation. Trends in Plant Science 10:269-274.

Bueno, J. M., P. Sáez-Plaza, F. Ramos-Escudero, A. M. Jiménez, F. R., and A. G. Asuero. 2012. Analysis and antioxidant capacity of anthocyanin pigments. Part ii: chemical structure, color, and intake of anthocyanins. Critical Reviews in Plant Sciences 42:126-151.

Burger, J. and G. E. Edwards. 1996. Photosynthetic efficiency, and photodamage by UV and visible radiation, in red versus green leaf Coleus varieties. Plant and Cell Physiology 37:395-399.

Burns, K. C. and J. L. Dalen. 2002. Foliage color contrasts and adaptive fruit color variation in a bird-dispersed plant community. Oikos 96:463-469.

Calogirou, A., B. R. Larsen, and D. Kotzias. 1999. Gas-phase terpene oxidation products: a review. Atmospheric Environment 33:1423-1439.

Calvo, D. and J. M. Molina. 2010. Differences in foliage affect performance of the lappet moth, Strblote panda: implications for species fitness. Journal of Insect Science 10:1-14.

Carraher, C., A. Authier, B. Steinwender, and R. D. Newcomb. 2012. Sequence comparisons of odorant receptorts among Tortricid moths reveal different rates of molecular evolution among family members. PLoS ONE 7:e38391.

Carroll, M. J., E. A. Schmelz, R. L. Meagher, and P. E. A. Teal. 2006. Attraction of Spodoptera frugiperda larvae to volatiles from herbivore-damaged maize seedlings. Journal of Chemical Ecology 32:1911-1924.

Chalker-Scott, L. 1999. Environmental significance of anthocyanins in plant stress responses. Photochemistry and Photobiology 70:1-9.

Chalker-Scott, L. 2002. Do anthocyanins function as osmoregulators in leaf tissues?in K. Gould and D. W. Lee, editors. Anthocyanins in Leaves. Academic Press, Amsterdam. Advances in Botanical Research; Vol. 37

Chittka, L. and T. F. Döring. 2007. Are autumn foliage colors red signals to aphids? PLoS Biology 5:e187.

Choinski Jr, J. S., P. Ralph, and D. Eamus. 2003. Changes in photosynthesis during leaf expansion in Corymbia gummifera. Australian Journal of Botany 51:111118.

Close, D. C. and C. L. Beadle. 2003. The ecophysiology of foliar anthocyanin. Botanical Review 69:149-161.

Close, D. C. and C. McArthur. 2002. Rethinking the role of many plant phenolics: protection from photodamage not herbivores? Oikos 99:166-172.

Coley, P. D. and T. M. Aide. 1989. Red coloration of tropical young leaves: a possible antifungal defence? Journal of Tropical Ecology 5:293-300.

Coley, P. D. and J. A. Barone. 1996. Herbivory and plant defence in tropical forests. Annual Review of Ecology, Evolution, and Systematics 27:305-335.

Coley, P. D., J. Lokvam, K. Rudolph, K. Bromberg, T. E. Sackett, L. Wright, T. Brenes-Arguedas, D. Dvorett, S. Ring, A. Clark, C. Baptiste, R. T. Pennington, and T. A. Kursar. 2005. Divergent defensive strategies of young leaves in two species of Inga. Ecology 86:2633-2643.

Cooney, L. J., J. W. van Klink, N. M. Hughes, N. B. Perry, H. M. Schaefer, I. J. Menzies, and K. S. Gould. 2012. Red leaf margins indicate increased polygodial content and function as visual signals to reduce herbivory in Pseudowintera colorata. New Phytologist.

Corbett, R. E. and P. K. Grant. 1958. The volatile oil of Pseudowintera colorata. 1. Journal of the Science of Food and Agriculture 9:733-739. 
Costa Freitas, A. M., C. Parreira, and L. Vilas-Boas. 2001. Comparison of two SPME fibres for differentiation of coffee by analysis of volatile compounds. Chromatographa 54:647-652.

D'Auria, J. C., E. Pichersky, A. Schaub, A. Hansel, and J. Gershenzon. 2007. Characterization of a BAHD acyltransferase responsible for producing the green leaf volatile (Z)-3-hexen-1-yl acetate in Arabidopsis thaliana. Plant Journal 49:194-207.

De Moraes, C. M., M. C. Mescher, and J. H. Tumlinson. 2001. Caterpillar-induced nocturnal plant volatiles repel conspecific females. Nature 410:577-580.

Delphia, C. M., M. C. Mescher, and C. M. De Moraes. 2007. Induction of plant volatiles by herbivores with different feeding habits and the effects of induced defenses on host-plant selection by thrips. Journal of Chemical Ecology 33:997-1012.

Delphia, C. M., J. R. Rohr, A. G. Stephenson, C. M. De Moraes, and M. C. Mescher. 2009. Effects of genetic variation and inbreeding on volatile production in a field population of horsenettle. International Journal of Plant Sciences 170:1220.

Demmig-Adams, B. and W. W. Adams III. 1992. Photoprotection and other responses of plants to high light stress. Annual review of Plant Physiology and Plant Molecular Biology 43:599-626.

Demmig-Adams, B. and W. W. Adams III. 1996. The role of xanthophyll cycle carotenoids in the protection of photosynthesis. Trends in Plant Science 1:2126.

Demmig-Adams, B. and W. W. Adams III. 2003. Photoinhibition. Pages 707-714 in T. B., D. Murphy, and B. Murray, editors. Encyclopaedia of applied plant science. Academic Press, London, UK.

Demmig-Adams, B. and W. W. Adams III. 2006. Photoprotection in an ecological context: the remarkable complexity of thermal energy dissipation. New Phytologist 172:11-21.

Diaz, C., V. Saliba-Colombani, O. Loudet, P. Belluomo, L. Moreau, F. DanielVedele, J.-F. Morot-Gaurdry, and C. Mascalaux-Daubresse. 2006. Leaf yellowing and anthocyanin accumulation are two genetically independent strategies in response to nitrogen limitation in Arabidopsis thaliana. Plant and Cell Physiology 47:74-83.

Dicke, M. and H. Dijkman. 2001. Within-plant circulation of systemic elicitor of induced defence and release from roots of elicitor that affects neighbouring plants. Biochemical Systematics and Ecology 29:1075-1087.

Dobler, S., G. Petschenka, and H. Pankoke. 2011. Coping with toxic plant compounds - the insect's perspective on iridoid glycosides and cardenolides. Phytochemistry 72:1593-1604.

Dodd, I. C., C. Critchley, G. S. Woodall, and G. R. Stewart. 1998. Photoinhibition in differently coloured juvelinle leaves of Syzygium species. Journal of Experimental Botany 49:1437-1445.

Dominy, N. J., P. W. Lucas, L. W. Ramsden, P. Riba-Hernandez, K. E. Stoner, and I. M. Turner. 2002. Why are young leaves red? Oikos 98:163-176.

Döring, T. F., M. Archetti, and J. Hardie. 2009. Autumn leaves seen through herbivore eyes. Proceedings of the Royal Society of London Series BBiological Sciences 276:121-127.

Döring, T. F. and L. Chittka. 2007. Visual ecology of aphids-a critical review on the role of colours in host finding. Arthropod-Plant Interactions 1:3-16. 
Dudareva, N., F. Negre, D. A. Nagegowda, and I. Orlova. 2006. Plant volatiles: Recent advances and future perspectives. Critical Reviews in Plant Sciences 25:417-440.

Dugdale, J. S. 1990. Reassessment of Ctenopseustis Meyrick and Planotortrix Dugdale with descriptions of two new genera (Lepidoptera: Tortricidae). New Zealand Journal of Zoology 17:437-465.

Ehleringer, J. R., I. Ullmann, O. L. Lange, G. D. Farquhar, I. R. Cowan, E. D. Schulze, and H. Ziegler. 1986. Mistletoes - a hypothesis concerning morphological and chemical avoidance of herbivory. Oecologia 70:234-237.

Esteban, R., B. Fernández-Marín, J. M. Becerril, and J. I. García-Plazaola. 2008. Photoprotective implications of leaf variegation in $E$. dens-canis L. and $P$. officinalis L. Journal of Plant Physiology 165:1255-1263.

Facelli, J. M. 1993. Experimental evaluation of the foliar flag hypothesis using fruits of Rhus glabra (L). Oecologia 93:70-72.

Fadzly, N. and K. C. Burns. 2010. Hiding from the ghost of herbivory past: evidence for crypsis in an insular tree species. International Journal of Plant Sciences 171:828-833.

Fadzly, N., C. Jack, H. M. Schaefer, and K. C. Burns. 2009. Ontogenetic colour changes in an insular tree species: signalling to extinct browsing birds? New Phytologist 184:495-501.

Feild, T. S., T. Brodribb, and N. M. Holbrook. 2002. Hardly a relict: freezing and the evolution of vesselless wood in Winteraceae. Evolution 56:464-478.

Feild, T. S., D. W. Lee, and N. M. Holbrook. 2001. Why leaves turn red in autumn. The role of anthocyanins in senescing leaves of red-osier dogwood. Plant Physiology 127:566-574.

Fineblum, W. L. and M. D. Rausher. 1997. Do floral pigmentation genes also influence resistance to enemies? The W locus in Ipomoea purpurea. Ecology 78:1646-1654.

Fondom, N. Y., S. Castro-Nava, and A. J. Huerta. 2009. Photoprotective mechanisms during leaf ontongeny: cuticular development and anthocyanin deposition in two morphs of Agave strata that differ in leaf coloration. Botany-Botanique 87:1186-1197.

Fornoni, J. 2011. Ecological and evolutionary implications of plant tolerance to herbivory. Functional Ecology 25:399-407.

Forsman, A., J. Ahnesjö, S. Caesar, and M. Karlsson. 2008. A model of ecological and evolutionary consequences of colour polymorphism. Ecology 89:34-40.

Fournier, V., J. A. Rosenheim, J. Brodeur, L. O. Laney, and M. W. Johnson. 2003. Herbivorous mites as ecological engineers: indirect effects on arthropods inhabiting papaya foliage. Oecologia 135:442-450.

Foyer, C. H. and G. Noctor. 2005. Oxidant and antioxidant signalling in plants: a reevaluation of the concept of oxidative stress in a physiological context. Plant Cell and Environment 28:1056-1071.

Foyer, C. H., G. Noctor, and H. F. Van Emden. 2007. An evaluation of the cost of making specific secondary metabolites: Does the yield penalty incurred by host plant resistance to insects result from competition for resources? International Journal of Pest Management 53:175-182.

Franceschi, V. R., P. Krokene, E. Chrisiansen, and T. Krekling. 2005. Anatomical and chemical defences of conifer bark against bark beetles and other pests. New Phytologist 167:353-375. 
Fred, M. S. and J. E. Brommer. 2010. Olfaction and vision in host plant location by Parnassius apollo larvae: consequences for survival and dynamics. Animal Behaviour 79:313-320.

Fukui, A. 2001. Indirect interactions mediated by leaf shelters in animal-plant communities. Population Ecology 43:31-40.

Futuyma, D. J. and A. A. Agrawal. 2009. Macroevolution and the biological diversity of plants and herbivores. Proceedings of the National Academy of Sciences 106:18054-18061.

Ganeteg, U., C. Kulheim, J. Andersson, and S. Jansson. 2004. Is each light-harvesting complex protein important for plant fitness? Plant Physiology 134:502-509.

Genty, B., J.-M. Briantais, and N. R. Baker. 1989. The relationship between the quantum yield of photosynthetic electron transport and quenching of chlorophyll fluorescence. Biochemica et Biophysica Acta 990:87-92.

Genty, B., J. Harbinson, J.-M. Briantais, and N. R. Baker. 1990. The relationship between non-photochemical quenching of chlorophyll fluorescence and the rate of photosystem 2 photochemistry in leaves. Photosynthesis Research 25:249-257.

Gerard, P. J., N. B. Perry, L. D. Ruf, and L. M. Foster. 1993. Antifeedant and insecticidal activity of compounds from Pseudowintera colorata (Winteraceae) on the webbing clothes moth, Tineola bisselliella (Lepidoptera: Tineidae) and the Australian carpet beetle, Anthrenocerus australis (Coleoptera: Dermestidae). Bulletin of Entomological Research 83:547-552.

Gibbs, G. W. 2001. Habitats and biogeography of New Zealand's Deinacidine and Tusked weta species.in L. H. Field, editor. The biology of wetas, king crickets and their allies. CABI Publishing New York.

Givnish, T. J. 1988. Adaptation to sun and shade: a whole-plant perspective. Australian Journal of Plant Physiology 15:63-92.

Givnish, T. J. 1990. Leaf mottling - relation to growth form and leaf phenology and possible role as camouflage. Functional Ecology 4:463-474.

Glover, B. J. and H. M. Whitney. 2010. Structural colour and iridescence in plants: the poorly studied relations of pigment colour. Annals of Botany 105:505-511.

Gog, L., M. R. Berenbaum, E. H. DeLucia, and A. R. Zangerl. 2005. Autotoxic effects of essential oils on photosynthesis in parsley, parsnip, and rough lemon. Chemoecology 15:115-119.

Gouinguene, S., T. Degen, and T. C. J. Turlings. 2001. Variability in herbivoreinduced odour emissions among maize cultivars and their wild ancestors (teosinte). Chemoecology 11:9-16.

Gould, K. S. 2004. Nature's Swiss army knife: The diverse protective roles of anthocyanins in leaves. Journal of Biomedicine and Biotechnology 2004:314320.

Gould, K. S., D. A. Dudle, and H. S. Neufeld. 2010. Why some stems are red: cauline anthocyanins shield photosystem II against high light stress. Journal of Experimental Botany 61:2707-2717.

Gould, K. S., D. N. Kuhn, D. W. Lee, and S. F. Oberbauer. 1995. Why leaves are sometimes red. Nature 378:241-242.

Gould, K. S. and D. W. Lee. 1996. Physical and ultrastructural basus of blue leaf iridescence in four Malaysian understory plants. American Journal of Botany 83:45-50. 
Gould, K. S., K. R. Markham, R. H. Smith, and J. J. Goris. 2000. Functional role of anthocyanins in the leaves of Quintinia serrata A. Cunn. Journal of Experimental Botany 51:1107-1115.

Gould, K. S., J. McKelvie, and K. R. Markham. 2002a. Do anthocyanins function as antioxidants in leaves? Imaging of $\mathrm{H} 2 \mathrm{O} 2$ in red and green leaves after mechanical injury. Plant, Cell and Environment 25:1261-1269.

Gould, K. S. and B. D. Quinn. 1999. Do anthocyanins protect leaves of New Zealand native species from UV-B? New Zealand Journal of Botany 37:175-178.

Gould, K. S., T. C. Vogelmann, T. Han, and M. J. Clearwater. 2002b. Profiles of photosynthesis within red and green leaves of Quintinia serrata. Physiologia Plantarum 116:127-133.

Grace, S. C., B. A. Logan, and W. W. Adams. 1998. Seasonal differences in foliar content of chlorogenic acid, a phenylpropanoid antioxidant, in Mahonia repens. Plant, Cell and Environment 21:513-521.

Grant, G. G., J. Guo, L. MacDonald, and M. D. Coppens. 2007. Oviposition responses of spruce budworm (Lepidoptera: Tortricidae) to host terpenes and green-leaf volatiles. The Canadian Entomologist 139:564-575.

Grant, G. G., K. L. Ryall, D. B. Lyons, and M. M. Abou-Zaid. 2010. Differential response of male and female emerald ash borers (Col., Buprestidae) to (Z)-3hexenol and manuka oil. Journal of Applied Entomology 134:26-33.

Greenwood, R. M. and I. A. E. Atkinson. 1977. Evolution of divaricating plants in New Zealand in relation to moa browsing. Proceedings of the New Zealand Ecological Society 24:21-33.

Griffiths, P. D. and A. M. Shelton. 2004. Evaluation of cabbage genotypes for thrips damage. Cruciferae Newsletter:77-78.

Gripenberg, S., P. J. Mayhew, M. Parnell, and T. Roslin. 2010. A meta-analysis of preference-performance relationships in phytophagous insects. Ecology Letters 13:383-393.

Guzmán, B., E. Narbona, and P. Vargas. 2011. Similar reproductive success of the two petal colour polymorphisms of Cistus ladanifer (Cistaceae). Plant Biosystems 145:931-937.

Hada, H., J. Hidema, M. Maekawa, and T. Kumagai. 2003. Higher amounts of anthocyanins and UV-absorbing compounds effectively lowered CPD photorepair in purple rice (Oryza sativa L.). Plant, Cell \& Environment 26:1691-1701.

Hagen, S. B., S. Debeausse, N. G. Yoccoz, and I. Folstad. 2004. Autumn coloration as a signal of tree condition. Proceedings of the Royal Society of London Series B-Biological Sciences 271:S184-S185.

Hagen, S. B., I. Folstad, and S. W. Jakobsen. 2003. Autumn colouration and herbivore resistance in mountain birch (Betula pubescens). Ecology Letters 6:807-811.

Hakes, A. S. and J. T. Cronin. 2011. Resistance and tolerance to herbivory in Solidago altissima (Asteraceae): genetic variability, costs, and selection for multiple traits. American Journal of Botany 98:1446-1455.

Hamilton, W. D. and S. P. Brown. 2001. Autumn tree colours as a handicap signal. Proceedings of the Royal Society of London Series B-Biological Sciences 268:1489-1493.

Harborne, J. B. 1967. Comparative biochemistry of the flavonoids. Academic Press, New York. 
Hare, J. D. 2007. Variation in herbivore and methyl jasmonate-induced volatiles among genetic lines of Datura wrightii. Journal of Chemical Ecology 33:2028-2043.

Hare, J. D. 2011. Ecological Role of Volatiles Produced by Plants in Response to Damage by Herbivorous Insects. Pages 161-180 in M. R. Berenbaum, R. T. Carde, and G. E. Robinson, editors. Annual Review of Entomology. Annual Reviews, Palo Alto. Annual Review of Entomology; Vol. 56

Hare, J. D. 2012. How insect herbivores drive the evolution of plants. Science 338:5051.

Hare, J. D. and E. Elle. 2004. Survival and seed production of sticky and velvety Datura wrightii in the field: a five-year study. Ecology 85:615-622.

Harris, M. O., S. P. Foster, T. Bittar, K. Ekanayake, K. Looij, and A. Howard. 1995. Visual behaviour of neonate larvae of the light brown apple moth. Entomologia Experimentalis Et Applicata 77:323-334.

Hatanaka, A. 1993. The biogeneration of green odour by green leaves. Phytochemistry 34:1289-1295.

Hatier, J. H. B. and K. S. Gould. 2008a. Anthocyanin function in vegetative organs.in K. S. Gould, K. Davies, and C. Winefield, editors. Anthocyanins: biosynthesis, functions and applications. Springer, New York.

Hatier, J. H. B. and K. S. Gould. 2008b. Foliar anthocyanins as modulators of stress signals. Journal of Theoretical Biology 253:625-627.

He, J. and M. M. Giusti. 2010. Anthocyanins: natural colorants with health-promoting properties. Annual Review of Food Science and Technology 1:163-187.

Henriques, F. S. 2009. Leaf chlorophyll fluorescence: background and fundamentals for plant biologists. Botanical Review 75:249-270.

Herms, D. A. and W. J. Mattson. 1992. The dilemma of plants: to grown or defend. The Quarterly Review of Biology 67:283-335.

Herrera, C. M. 1991. Dissecting factors responsible for individual variation in plant fecundity. Ecology 72:1436-1448.

Herrera, C. M. 2009. Multiplicity in Unity: Plant subindividual variation \& interactions with animals. The University of Chicago Press, Chicago.

Hildebrand, D. F., G. C. Brown, D. M. Jackson, and T. R. Hamilton-Kemp. 1993. Effects of some leaf-emitted volatile compounds on aphid population increase. Journal of Chemical Ecology 19:1875-1887.

Himanen, S. J., J. D. Blande, T. Klemola, J. Pulkkinen, J. Heijari, and J. K. Holopainen. 2010. Birch (Betula spp.) leaves adsorb and re-release volatiles specific to neighbouring plants - a mechanism for associational herbivore resistance? New Phytologist 186:722-732.

Hoch, H. C., C. Pratt, and G. A. Marx. 1980. Subepidermal air spaces: basis for the phenotypic expression of the Argentum mutant of Pisum. American Journal of Botany 67:905-911.

Hoch, W. A., E. L. Singsaas, and B. H. McCown. 2003. Resorption protection. Anthocyanins facilitate nutrient recovery in Autumn by shielding leaves from potentially damaging light levels. Plant Physiology 133:1296-1305.

Hoch, W. A., E. L. Zeldin, and B. H. McCown. 2001. Physiological significance of anthocyanins during autumnal leaf senescence. Tree Physiology 21:1-8.

Holopainen, J. K. 2004. Multiple functions of inducible plant volatiles. Trends in Plant Science 9:529-533.

Holopainen, J. K. 2008. Importance of olfactory and visual signals of autumn leaves in the coevolution of aphids and trees. Bioessays 30:889-896. 
Holopainen, J. K., J. Heijari, E. Oksanen, and G. A. Alessio. 2010. Leaf volatile emissions of Betula pendula during autumn coloration and leaf fall. Journal of Chemical Ecology 36:1068-1075.

Holopainen, J. K. and P. Peltonen. 2002. Bright autumn colours of deciduous trees attract aphids: nutrient retranslocation hypothesis. Oikos 99:184-188.

Honda, K. 1995. Chemical basis of differential oviposition by lepidopterous insects. Archives of Insect Biochemistry 30:1-23.

Hoque, E. and G. Remus. 1999. Natural UV-screening mechanisms of Norway Spruce (Picea abies [L.] Karst.) needles. Photochemistry and Photobiology 69:177-192.

Huang, C. H., F. M. Yan, J. A. Byers, J. R. Wang, and C. R. Xu. 2009. Volatiles induced by the larvae of the Asian corn borer (Ostrinia furnacalis) in maize plants affect behaviour of conspecific larvae and females adults. Insect Science 16:311-320.

Huber, D. P. W. and J. Bohlmann. 2004. Terpene synthases and the mediation of plant-insect ecological interations by terpenoids: a mini-review. Pages 70-81 in Q. C. B. Cronck, J. Whitton, R. H. Ree, and I. E. P. Taylor, editors. Plant adaptation: molecular genetics and ecology. NRC Research Press, Ottawa, Ontario.

Hughes, N. M. 2011. Winter leaf reddening in 'evergreen' species. New Phytologist 190:573-581.

Hughes, N. M., K. O. Burkey, J. Cavender-Bares, and W. K. Smith. 2012a. Xanthophyll cycle pigment and antioxidant profiles of winter-red (anthocyanic) and winter-green (acyanic) angiosperm evergreen species. Journal of Experimental Biology 63:1895-1905.

Hughes, N. M., K. L. Carpenter, and J. G. Cannon. 2012b. Estimating contribution of anthocyanin pigments to osmotic adjustment during winter leaf reddening. Journal of Plant Physiology 170:230-233.

Hughes, N. M., C. B. Morley, and W. K. Smith. 2007. Coordination of anthocyanin decline and photosynthetic maturation in juvenile leaves of three deciduous tree species. New Phytologist 175:675-685.

Hughes, N. M., H. S. Neufeld, and K. O. Burkey. 2005. Functional role of anthocyanins in high-light winter leaves of the evergreen herb Galax urceloata. New Phytologist 168:575-587.

Hughes, N. M., K. Reinhardt, T. S. Feild, A. R. Gerardi, and W. K. Smith. 2010a. Association between winter anthocyanin production and drought stress in angiosperm evergreen species. Journal of Experimental Botany 61:1699-1709.

Hughes, N. M. and W. K. Smith. 2007a. Attenuation of incident light in Galax urceolata (Diapensiaceae): concerted influence of adaxial and abaxial anthocyanic layers on photoprotection. American Journal of Botany 94:784790.

Hughes, N. M. and W. K. Smith. 2007b. Seasonal photosynthesis and anthocyanin production in 10 broadleaf evergreen species. Functional Plant Biology 34:1072-1079.

Hughes, N. M., W. K. Smith, and K. S. Gould. 2010b. Red (anthocyanic) leaf margins do not correspond to increased phenolic content in New Zealand Veronica spp. Annals of Botany 105:647-654.

Hughes, N. M., T. C. Vogelmann, and W. K. Smith. 2008. Optical effects of abaxial anthocyanin on absorption of red wavelengths by understorey species: 
revisiting the back-scatter hypothesis. Journal of Experimental Botany 59:3435-3442.

IOFI Working Group on Methods of Analysis. 2010. Guidelines for solid-phase micro-extraction (SPME) of volatile flavour compounds for gaschromatographic analysis, from the Working Group on Methods of Analysis of the International Organization of the Flavor Industry (IOFI). Flavour and Fragrance Journal 25:404-406.

Jankowska, B. 2006. The occurance of some Lepidoptera pests on different cabbage vegetables. Journal of Plant Protection Research 46:181-190.

Janzen, D. H. 1980. When is it coevolution? Evolution 34:611-612.

Johnson, E. T., M. A. Berhow, and P. F. Dowd. 2008. Colored and white sectors from star-patterned petunia flowers display differential resistance to corn earworm and cabbage looper larvae. Journal of Chemical Ecology 34:757-765.

Jordan, M. D., A. Anderson, D. Begum, C. Carraher, A. Authier, S. D. G. Marshall, A. Kiely, L. N. Gatehouse, D. R. Greenwood, D. L. Christie, A. V. Kralicek, S. C. Trowell, and R. D. Newcomb. 2009. Odorant receptors from the Light brown apple moth (Epiphyas postvittana) recognize important volatile compounds produced by plants. Chemical Senses 34:383-394.

Juniper, B. E. 1993. Flamboyant flushes: a reinterpretation of non-green flush colours in leaves. Pages 49-57 International Dendrological Society Yearbook.

Karageorgou, P., C. Buschmann, and Y. Manetas. 2008. Red leaf color as a warning signal against insect herbivory: Honest or mimetic? Flora 203:648-652.

Karageorgou, P. and Y. Manetas. 2006. The importance of being red when young: anthocyanins and the protection of young leaves of Quercus coccifera from insect herbivory and excess light. Tree Physiology 26:613-621.

Kawagoe, T., K. K. Shimizu, T. Kakutani, and H. Kudoh. 2011. Coexistence of trichome variation in a natural plant population: a combined study using ecological and candidate gene approaches. PLoS ONE 6:e22184.

Keeling, C. I. and J. Bohlmann. 2006. Genes, enzymes and chemicals of terpenoid diversity in the constitutive and induced defence of conifers against insects and pathogens. New Phytologist 170:657-675.

Keskitalo, J., G. Berquist, P. Gardeström, and S. Jansson. 2005. A cellular timetable of autumn senescence. Plant Physiology 139:1635-1648.

Kessler, A. and I. T. Baldwin. 2001. Defensive function of herbivore-induced plant volatile emissions in nature. Science 291:2141-2144.

Klooster, M. R., D. L. Clark, and T. M. Culley. 2009. Cryptic bracts facilitate herbivore avoidance in the mycoheterotrophic plant Monotropsis odorata (Ericaceae). American Journal of Botany 96:2197-2205.

Koricheva, J. 2002. Meta-analysis of sources of variation in fitness costs of plant antiherbivore defenses. Ecology 83:176-190.

Koziel, J., M. Jia, A. Khaled, J. Noah, and J. Pawliszyn. 1999. Field air analysis with SPME device. Analytica Chimica Acta 400:153-162.

Krol, M., G. R. Gray, N. P. A. Huner, V. M. Hurry, G. Öquist, and L. Malek. 1995. Low-temperature stress and photoperiod affect an increased tolerance to photoinhibition in Pinus bansiana seedlings. Canadian Journal of Botany 73:1119-1127.

Kyparissis, A., G. Grammatikopoulos, and Y. Manetas. 2007. Leaf morphological and physiological adjustments to the spectrally selective shade imposed by anthocyanins in Prunus cerasifera. Tree Physiology 27:849-857. 
Kytridis, V. P., P. Karageorgou, E. Levizou, and Y. Manetas. 2008. Intra-species variation in transient accumulation of leaf anthocyanins in Cistus creticus during winter: Evidence that anthocyanins may compensate for an inherent photosynthetic and photoprotective inferiority of the red-leaf phenotype. Journal of Plant Physiology 165:952-959.

Kytridis, V. P. and Y. Manetas. 2006. Mesophyll versus epidermal anthocyanins as potential in vivo antioxidants: evidence linking the putative antioxidant role to the proximity of oxy-radical source. Journal of Experimental Botany 57:22032210.

Lan, J., A. Li, and C. Chen. 2011. Effect of transient accumulation of anthocyanin on leaf development and photoprotection of Fagopyrum dibotrys mutant. Biologia Plantarum 55:766-770.

Landolt, P. J., J. A. Brumley, C. L. Smithhisler, L. L. Biddick, and R. W. Hofstetter. 2000. Apple fruit infested with codling moth are more attractive to neonate codling moth larvae and possess increased amounts of $(E, E)$ - $\alpha$-farnesene. Journal of Chemical Ecology 26:1685-1699.

Langhoff, P., A. Authier, T. R. Buckley, J. S. Dugdale, A. Rodrigos, and R. D. Newcomb. 2009. DNA barcoding of the endemic New Zealand leafroller moth genera, Ctenopseustis and Planotortrix. Molecular Ecology Resources 9:691698.

Laothawornkitkul, J., N. D. Paul, C. E. Vickers, M. Possell, J. E. Taylor, P. M. Mullineaux, and C. N. Hewitt. 2008. Isoprene emissions influence herbivore feeding decisions. Plant, Cell and Environment 31:1410-1415.

Lawler, I. R., B. M. Eschler, D. M. Schliebs, and W. J. Foley. 1999. Relationship between chemical functional groups on Eucalyptus secondary metabolites and their effectiveness as marsupial antifeedants. Journal of Chemical Ecology 25:2561-2573.

Lawler, I. R., W. J. Foley, B. M. Eschler, D. M. Pass, and K. Handasyde. 1998. Intraspecific variation in Eucalyptus secondary metabolites determines food intake by folivorous marsupials. Oecologia 116:160-169.

Lee, D. W. 2002. Anthocyanins in leaves: Distribution, phylogeny and development. Pages 37-53 Advances in Botanical Research, Vol 37. Academic Press Ltd, London. Advances in Botanical Research Incorporating Advances in Plant Pathology; 37

Lee, D. W. 2007. Nature's palette. The University of Chicago Press, Chicago and London.

Lee, D. W., S. Brammeier, and A. P. Smith. 1987. The selective advantages of anthocyanins in developing leaves of mango and cacao. Biotropica 19:40-49.

Lee, D. W. and T. M. Collins. 2001. Phylogenetic and ontogenetic influences on the distribution of anthocyanins and betacyanins in leaves of tropical plants. International Journal of Plant Sciences 162:1141-1153.

Lee, D. W. and K. S. Gould. 2002a. Anthocyanins in leaves and other vegetative organs: An introduction. Pages 1-16 Advances in Botanical Research, Vol 37. Academic Press Ltd, London. Advances in Botanical Research Incorporating Advances in Plant Pathology; 37

Lee, D. W. and K. S. Gould. 2002b. Why leaves turn red - Pigments called anthocyanins probably protect leaves from light damage by direct shielding and by scavenging free radicals. American Scientist 90:524-531.

Lee, D. W. and J. B. Lowry. 1980. Young-leaf anthocyanin and solar ultraviolet. Biotropica 12:75-76. 
Lee, D. W., J. B. Lowry, and B. C. Stone. 1979. Abaxial anthocyanin layer in leaves of tropical rain forest plants: enhancer of light capture in deep shade. Biotropica 11:70-77.

Lee, D. W., J. O'Keefe, N. M. Holbrook, and T. S. Feild. 2003. Pigment dynamics and autumn leaf senescence in a New England deciduous forest, eastern USA. Ecological Research 18:677-694.

Leimu, R. and J. Koricheva. 2006. A meta-analysis of tradeoffs between plant tolerance and resistance to herbivores: combining the evidence from ecological and agricultural studies. Oikos 112:1-9.

Lester, P. J. and A. M. Barrington. 1997. Gamma irradiation for postharvest disinfestation of Ctenopseustis obliquana (Walker) (Lep., Tortricidae). Journal of Applied Entomology 121:107-110.

Lev-Yadun, S. 2006a. Defensive coloration in plants: a review of current ideas about anti-herbivore coloration strategies.

Lev-Yadun, S. 2006b. Defensive functions of white coloration in coastal and dune plants. Israel Journal of Plant Sciences 54:317-325.

Lev-Yadun, S., A. Dafni, M. A. Flaishman, M. Inbar, I. Izhaki, G. Katzir, and G. Ne'eman. 2004. Plant coloration undermines herbivorous insect camouflage. Bioessays 26:1126-1130.

Lev-Yadun, S. and K. S. Gould. 2007. What do red and yellow autumn leaves signal? Botanical Review 73:279-289.

Lev-Yadun, S. and K. S. Gould. 2009. Role of anthocyanins in plant defence. Pages 21-48 in K. Gould, K. Davies, and C. Winefield, editors. Anthocyanins: Biosynthesis, Functions, and Applications. Springer, New York.

Lev-Yadun, S. and J. K. Holopainen. 2009. Why red-dominated autumn leaves in America and yellow-dominated autumn leaves in Northern Europe? New Phytologist 183:506-512.

Lev-Yadun, S., M. Inbar, I. Izhaki, G. Ne'eman, and A. Dafni. 2002. Colour patterns in vegetative parts of plants deserve more research attention. Trends in Plant Science 7:59-60.

Levene, H. 1953. Genetic equilibrium when more than one eclogical niche is available. The American Naturalist 87:331-333.

Levine, T. R. and C. R. Hullett. 2002. Eta squared, partial eta squared, and misreporting of effect size in communication research. Human Communication Research 28:612-625.

Li, P., S. Castagnoli, and L. Cheng. 2008. Red 'Anjou' pear has higher photoprotective capacity than green 'Anjou'. Physiologia Plantarum 134:486-498.

Li, P. M., L. Cheng, T. Peng, and H. Y. Gao. 2009. CO2 assimilation and chlorophyll fluorescence in green versus red Berberis thunbergii leaves measured with different quality irradiation. Photosynthetica 47:11-18.

Li, X., J. M. Baskin, and C. C. Baskin. 1999. Contrasting dispersal phenologies in two fleshy-fruited congeneric shrubs, Rhus aromatica Ait. and Rhus glabra L. (Anacardiaceae). Canadian Journal of Botany-Revue Canadienne De Botanique 77:976-988.

Liakopoulos, G., D. Nikolopoulos, A. Klouvatou, K.-A. Vekkos, Y. Manetas, and G. Karabourniotis. 2006. The photoprotective role of epidermal anthocyanins and surface pubescence in young leaves of grapevine (Vitis vinifera). Annals of Botany 98:257-265. 
Liakopoulos, G. and I. Spanorigas. 2012. Foliar anthocyanins in Pelargonium x hortorum are unable to alleviate light stress under photoinhibitory conditions. Phytosynthetica 50:254-262.

Lichtenthaler, H. K. 1987. Chlorophyll and carotenoids: pigments of photosynthetic biomembranes. Methods in Enzymology 148:350-385.

Long, S. P., S. Humphries, and P. G. Falkowski. 1994. Photoinhibition of photosynthesis in nature. Annual review of Plant Physiology and Plant Molecular Biology 45:633-662.

Loughrin, J. H., A. Manukian, R. R. Heath, T. C. J. Turlings, and J. H. Tumlinson. 1994. Diurnal cycle of emissions of induced volatile terpenoids by herbivoreinjured cotton plants. Proceedings of the National Academy of Sciences 91:11836-11840.

Magsig-Castillo, J., J. G. Morse, G. P. Walker, J. L. Bi, P. F. Rugman-Jones, and R. Stouthamer. 2012. Phoretic dispersal of armored scale crawlers (Hemiptera: Diaspididae). Journal of Economic Entomology 103:1172-1179.

Majer, J. D., H. Recher, and N. Keals. 1996. Branchlet shaking: a method for sampling tree canopy arthopods under windy conditions. Australian Journal of Ecology 21:229-234.

Manetas, Y. 2006. Why some leaves are anthocyanic and why most anthocyanic leaves are red? Flora 201:163-177.

Manetas, Y., A. Drinia, and Y. Petropoulou. 2002. High contents of anthocyanins in young leaves are correlated with low pools of xanthophyll cycle components and low risk of photoinhibition. Photosynthetica 40:349-354.

Manetas, Y., Y. Petropoulou, G. K. Psaras, and A. Drinia. 2003. Exposed red (anthocyanic) leaves of Quercus coccifera display shade characteristics. Functional Plant Biology 30:265-270.

Mäntylä, E., G. A. Alessio, J. D. Blande, J. Heijari, J. K. Holopainen, T. Laaksonsen, P. Piirtola, and T. Klemola. 2008. From plants to birds: higher avian predation rates in trees responding to insect herbivory. PLoS ONE 3:e2832.

Markwick, N. P., J. Poulton, R. V. Espley, D. D. Rowan, T. K. McGhie, G. Wadasinghe, M. Wohlers, Y. Jia, and A. C. Allan. 2012. Red-foliaged apples affect the establishment, growth, and development of the light brown apple moth, Epiphyas postvittana. Entomologia Experimentalis Et Applicata 146:261-275.

Marquis, R. J. 1984. Leaf herbivores decrease fitness of a tropical plant. Science 226:537-539.

Martinsen, G. D., K. D. Floate, A. M. Waltz, G. M. Wimp, and T. G. Whitham. 2000. Positive interactions between leafrollers and other arthropods enhance biodiversity on hybrid cottonwoods. Oecologia 123:82-89.

Matile, P. 2000. Biochemistry of Indian summer: physiology of autumnal leaf coloration. Experimental Gerontology 35:145-158.

Mattson, W. J. 1980. Herbivory in relation to plant nitrogen content. Annual Review of Ecology and Systematics 11:119-161.

Maxwell, K. and G. N. Johnson. 2000. Chlorophyll fluorescence - a practical guide. Journal of Experimental Botany 51:659-668.

Maynard Smith, J. and D. G. C. Harper. 1995. Animal signals: models and terminology. Journal of Theoretical Biology 177:305-311.

Maynard Smith, J. and D. G. C. Harper. 2003. Animal signals: models and terminology. Oxford University Press, Oxford. 
McGlone, M. S. and W. W. Topping. 1977. Ananuian (post-glacial) pollen diagrams from the Tongariro region, North Island, New Zealand. New Zealand Journal of Botany 15:749-760.

McGraw, J. B. and H. Caswell. 1996. Estimation of individual fitness from lifehistory data. The American Naturalist 147:47-64.

McKinnon, J. S. and M. E. R. Pierotti. 2010. Colour polymorphism and correlated characters: genetic mechanisms and evolution. Molecular Ecology 19:51015125.

Mendez, M., D. Gwynn Jones, and Y. Manetas. 1999. Enhanced UV-B radiation under field conditions increased anthocyanin and reduces the risk of photoinhibition but dies not affect growth in the carnivorous plant Pinguicula vulgaris. New Phytologist 144:275-282.

Merzlyak, M. N., O. B. Chivkunova, A. E. Solovchenko, and K. R. Naqvi. 2008. Light absorption by anthocyanins in juvenile, stressed, and senescing leaves. Journal of Experimental Botany 59:3903-3911.

Meyers, L. S., G. Gamst, and A. J. Guarino. 2006. Applied multivariate research: design and interpretation. Sage Publications, Inc., Thousand Oaks.

Mittler, R. 2002. Oxidative stress, antioxidants and stress tolerance. Trends in Plant Science 7:405-410.

Mol, J., G. I. Jenkins, E. Shafer, and D. Weiss. 1996. Signal perception, transduction and gene expression involved in anthocyanin biosynthesis. Critical Reviews in Plant Sciences 15:525-557.

Mole, S. 1994. Trade-offs and constraints in plant-herbivore defense theory: a lifehistory perspective. Oikos 71:3-12.

Morgan-Richards, M. and G. W. Gibbs. 2001. A phylogenetic analysis of New Zealand giant and tree weta (Orthoptera: Anostostomatidae: Deinacrida and Hemideina) using morphological and genetic characters. Invertebrate Taxonomy 15:1-12.

Müller, H. J. 1964. The frequency of aphid landings on coloured lettuce plants. Entomologia Experimentalis Et Applicata 7:85-104.

Nabity, P. D., J. A. Zavala, and E. H. DeLucia. 2009. Indirect suppression of photosynthesis on individual leaves by arthropod herbivory. Annals of Botany 103:655-663.

Nakamura, M. and T. Ohgushi. 2003. Positive and negative effects of leaf shelters on herbivorous insects: linking multiple herbivore species on a Willow. Oecologia 136:445-449.

Natale, D., L. Mattiacci, A. Hern, E. Pasqualini, and S. Dorn. 2003. Response of female Cydia molesta (Lepidotpera : Tortricidae) to plant derived volatiles. Bulletin of Entomological Research 93:335-342.

Natale, D., L. Mattiacci, A. Hern, E. Pasqualini, and S. Dorn. 2004. Bioassay approaches to observing behavioural responses of adult female Cydia molesta to host plant odour. Journal of Applied Entomology 128:182-187.

Neill, S. O. 2002. The fuctional role of anthocyanins in leaves. University of Auckland, Auckland, NZ.

Neill, S. O. and K. S. Gould. 1999. Optical properties of leaves in relation to anthocyanin concentration and distribution. Canadian Journal of BotanyRevue Canadienne De Botanique 77:1777-1782.

Neill, S. O. and K. S. Gould. 2003. Anthocyanins in leaves: light attenuators or antioxidants? Functional Plant Biology 30:865-873. 
Neill, S. O., K. S. Gould, P. A. Kilmartin, K. A. Mitchell, and K. R. Markham. $2002 a$. Antioxidant activities of red versus green leaves in Elatostema rugosum. Plant, Cell and Environment 25:539-547.

Neill, S. O., K. S. Gould, P. A. Kilmartin, K. A. Mitchell, and K. R. Markham. 2002b. Antioxidant capacities of green and cyanic leaves in the sun species, Quintinia serrata. Functional Plant Biology 29:1437-1443.

Nielsen, S. and A. Simonsen. 2011. Photosynthesis and photoinhibition in two differently coloured varieties of Oxalis triangularis - the effect of anthocyanin content. Photosynthetica 49:346-352.

Nikiforou, C. and Y. Manetas. 2010. Strength of winter leaf redness as an indicator of stress vunerable individuals in Pistacia lentiscus. Flora 205:424-427.

Nikiforou, C., D. Nikolopoulos, and Y. Manetas. 2011. The winter-red-leaf syndrome in Pistacia lentiscus: Evidence that the anthocyanic phenotype suffers from nitrogen deficiency, low carboxylation efficiency and high risk of photoinhibition. Journal of Plant Physiology 168:2184-2187.

Nikiforou, C., K. Zeliou, V. P. Kytridis, A. Kyzeridou, and Y. Manetas. 2010. Are red leaf phenotypes more or less fit? The case of winter leaf reddening in Cistus creticus. Environmental and Experimental Botany 67:509-514.

Nishio, J. N. 2000. Why are higher plants green? Evolution of the higher plant photosynthetic pigment complement. Plant, Cell and Environment 23:539548.

Norton, S. A. 1980. Reproductive ecology of Pseudowintera (Winteraceae). Victoria University of Wellington, Wellington, New Zealand.

Novak, A. B. and F. T. Short. 2010. Leaf reddening in seagrasses. Botanica Marina 53:93-97.

Novak, A. B. and F. T. Short. 2011. Leaf reddening in the seagrass Thalassia testudinum in relation to anthocyanins, seagrass physiology and morphology, and plant protection. Marine Biology 158:1403-1416.

Núñez-Farfán, J., J. Fornoni, and P. L. Valverde. 2007. The evolution of resistance and tolerance to herbivores. Annual Review of Ecology, Evolution, and Systematics 38:541-566.

Ohgushi, T. 2005. Indirect interaction webs: herbivore-induced effects through trait change in plants. Annual Review of Ecology, Evolution, and Systematics 36:81-105.

Otte, D. 1974. Effects and functions in the evolution of signaling systems. Annual Review of Ecology and Systematics 5:385-417.

Ougham, H. J., P. Morris, and H. Thomas. 2005a. The colors of autumn leaves as symptoms of cellular recycling and defenses against environmental stresses. Current Topics in Developmental Biology 66:135-160.

Ougham, H. J., P. Morris, and H. Thomas. 2005b. The colors of autumn leaves as symptoms of cellular recycling and defenses against environmental stresses. Pages 135-160 Current Topics in Developmental Biology, Vol 66. Elsevier Academic Press Inc, San Diego. Current Topics in Developmental Biology; 66

Page, J. E. and G. H. N. Towers. 2002. Anthocyanins protect light-sensitive thiarubrine phototoxins. Planta 215:478-484.

Paré, P. W. and J. H. Tumlinson. 1997. De novo biosynthsis of volatiles induced by insect herbivory in cotton plants. Plant Physiology 114:1161-1167.

Pareja, M., A. Mohib, M. A. Birkett, S. Dufour, and R. T. Glinwood. 2009. Multivariate statistics coupled to generalized linear models reveal complex use of chemical cues by a parasitoid. Animal Behaviour 77:901-909. 
Peng, M., D. Hudson, A. Schofield, R. Tsao, R. Yang, H. Gu, Y.-M. Bi, and S. J. Rothstein. 2008. Adaptation of Arabidopsis to nitrogen limitation involved induction of anthocyanin synthesis which is controlled by the NLA gene. Journal of Experimental Botany 59:2933-2944.

Perkins, L. E., B. W. Cribb, J. Hanan, E. Glaze, C. Beveridge, and M. Zaluki. 2008. Where to from here? The mechanisms enabling the movement of first instar caterpillars on whole plants using Helicoverpa armigera (Hübner). ArthropodPlant Interactions 2:197-207.

Perry, N. B., E. J. Burgess, S. D. Lorimer, and J. W. van Klink. 1996. Fatty acid anilides as internal standards for high performance liquid chromatographic analyses of Valeriana officinalis L. and other medicinal plants. Phytochemical Analysis 7:263-268.

Philpott, M., L. R. Ferguson, K. S. Gould, and P. J. Harris. 2009. Anthocyanidincontaining compounds occur in the periderm cell walls of the storage roots of sweet potato (Ipomoea batatas). Journal of Plant Physiology 166:1112-1117.

Piesik, D., D. Rochat, J. van der Pers, and F. Marion-Poll. 2009. Pulsed odors from maize or spinach elicit orientation in European corn borer neonate larvae. Journal of Chemical Ecology 35:1032-1042.

Pietrini, F., M. A. Iannelli, and A. Massacci. 2002. Anthocyanin acculumation in the illuminated surface of maize leaves enhances protection from photo-inhibitory risks at low temperature, without further limitation to photosynthesis. Plant, Cell and Environment 25:1251-1259.

Pietrini, F. and A. Massacci. 1998. Leaf anthocyanin content changes in Zea mays L. grown at low temperature: Significance for the relationship between the quantum yield of PS II and the apparent quantum yield of $\mathrm{CO} 2$ assimilation. Photosynthesis Research 58:213-219.

Pole, M. 2007. Plant-macrofossil assemblages during Pliocene uplift, South Island, New Zealand. Australian Journal of Botany 55:118-142.

Powell, G., J. Hardie, and J. A. Pickett. 1993. Effects of the antifeedant polygodial on plant penetration by aphids, assessed by video and electrical recording. Entomologia Experimentalis Et Applicata 68:193-200.

Powell, G., J. Hardie, and J. A. Pickett. 1995. Responses of Myzus persicae to the repellent polygodial in choice and no-choice video assays with young and mature leaf tissue. Entomologia Experimentalis Et Applicata 74:91-94.

Primack, R. B. and H. Kang. 1989. Measuring fitness and natural selection in wild plant populations. Annual Review of Ecology and Systematics 20:367-396.

Pszczolkowski, M. A. and J. J. Brown. 2005. Single experience learning of host fruit selection by lepidopteran larvae. Physiology \& Behavior 86:168-175.

R Core Team. 2013. R: a language and environment for statistical computing. R Foundation for Statistical Computing. http://www.R-project.org, Vienna, Austria.

Ramirez, C. C., B. Lavandero, and M. Archetti. 2008. Coevolution and the adaptive value of autumn tree colours: colour preference and growth rates of a southern beech aphid. Journal of Evolutionary Biology 21:49-56.

Reeves, J. L. 2011. Vision should not be overlooked as an important sensory modality for finding host plants. Environmental Entomology 40:855-863.

Regier, J. C., J. W. Brown, C. Mitter, J. Baixeras, S. Cho, M. P. Cummings, and A. Zwick. 2012. A molecular phylogeny for the leaf-roller moths (Lepidoptera: Tortricidae) and its implications for classification and life history evolution. PLoS ONE 7:e35574. 
Rice-Evans, C. A., N. J. Miller, and G. Paganga. 1997. Antioxidant properties of phenolic compounds. Trends in Plant Science 2:152-159.

Roessingh, P., S. Xu, and S. B. J. Menken. 2007. Olfactory receptors on the maxillary palps of small ermine moth larvae: evolutionary history of benzaldehyde sensitivity. Journal of Comparative Physiology A 193:635-647.

Rolshausen, G. and H. M. Schaefer. 2007. Do aphids paint the tree red (or yellow) can herbivore resistance or photoprotection explain colourful leaves in autumn? Plant Ecology 191:77-84.

Rosenthal, J. P. and P. M. Kotanen. 1994. Terrestrial plant tolerance to herbivory. Trends in Ecology \& Evolution 9:145-148.

Ruther, J. and S. Kleier. 2005. Plant-plant signalling: Ethylene synergizes volatile emission in Zea mays induced by exposure to (Z)-3-hexen-1-ol. Journal of Chemical Ecology 31:2217-2222.

Saito, K. and M. Yamazaki. 2002. Biochemistry and molecular biology of the latestage of biosynthesis of anthocyanin: lessons from Perilla frutescens as a model plant. New Phytologist 155:9-23.

Salgado-Luarte, C. and E. Gianoli. 2010. Herbivory on temperate rainforest seedlings in sun and shade: resistance, tolerance and habitat distribution. PLoS ONE 5:e11460.

Salloum, A., V. Colson, and F. Marion-Poll. 2011. Appetitive and aversive learning in Spodoptera littoralis larvae. Chemical Senses 36:725-731.

Salmon, J. T. 1991. The stick insects of New Zealand. Reed Books, Auckland.

Sampson, F. B. 1980. Natural hybridism in Pseudowintera (Winteraceae). New Zealand Journal of Botany 18:43-51.

Sanger, J. E. 1971. Quantitative investigations of leaf pigments from their inception in buds through autumn coloration to decomposition in falling leaves. Ecology 52:1075-\&.

Schaberg, P. G., A. K. van den Berg, P. F. Murakami, J. B. Shane, and J. R. Donnelly. 2003. Factors infuencing red expression in autumn foliage of sugar maple trees. Tree Physiology 23:325-333.

Schaefer, H. M. and K. S. Gould. 2007. Modelling the evolution of leaf colouration with binary assumptions is barking up the wrong tree. Journal of Theoretical Biology 249:638-639.

Schaefer, H. M., M. Rentzsch, and M. Breuer. 2008. Anthocyanins reduce fungal growth in fruits. Natural Product Communications 3:1267-1272.

Schaefer, H. M. and G. Rolshausen. 2006. Plants on red alert: do insects pay attention? Bioessays 28:65-71.

Schaefer, H. M. and G. Rolshausen. 2007a. Aphids do not attend to leaf colour as visual signal, but to the handicap of reproductive investment. Biology Letters 3:1-4.

Schaefer, H. M. and G. Rolshausen. 2007b. How to investigate a putative signal? Stick to the right method when assessing the response of a receiver. Biology Letters 3:152-153.

Schaefer, H. M. and G. D. Ruxton. 2011. Plant-animal communication. Oxford University Press Inc., New York.

Schaefer, H. M. and D. M. Wilkinson. 2004. Red leaves, insects and coevolution: a red herring? Trends in Ecology \& Evolution 19:616-618.

Schoonhoven, L. M., J. J. A. van Loon, and M. Dicke. 2005. Insect-plant biology. 2nd edition. Oxford University Press, Oxford. 
Schuman, M. C., N. Heinzel, E. Gaquerel, A. Svatos, and I. T. Baldwin. 2009. Polymorphism in jasmonate signaling partially accounts for the variety of volatiles produced by Nicotiana attenuata plants in a native population. New Phytologist 183:1134-1148.

Scott-Phillips, T. C. 2008. Defining biological communication. Journal of Evolutionary Biology 21:387-395.

Sheue, C.-R., S.-H. Pao, L.-F. Chien, P. Chesson, and C.-I. Peng. 2012. Natural foliar variegation without costs? The case of Begonia. Annals of Botany 109:10651075.

Simms, E. L. 1992. Costs of plant resistance to herbivory.in R. S. Fritz and E. L. Simms, editors. Plant resistance to herbivores and pathogens: ecology, evolution and genetics. University of Chicago Press, Chicago, Illinois, USA.

Simpson, S. J. and D. Raubenheimer. 2001. The geometric analysis of nutrientallelochemical interactions: a case study using locusts. Ecology 82:422-439.

Singh, P. 1983. A general purpose laboratory diet mixture for rearing insects. Insect Science and its Application 4:357-362.

Sinkkonen, A. 2006a. Do autumn leaf colours serve as a reproductive insurance against sucking herbivores? Oikos 113:557-562.

Sinkkonen, A. 2006b. Sexual reproduction advances autumn leaf colours in mountain birch (Betula pubescens sp. czerepanovii). Journal of Evolutionary Biology 19:1722-1724.

Sinkkonen, A. 2008. Red reveals branch die-back in Norway maple Acer platanoides. Annals of Botany 102:361-366.

Sinkkonen, A., E. Somerkoski, U. Paaso, J. K. Holopainen, M. Rousi, and J. Mikola. 2012. Genotypic variation in yellow autumn leaf colours explains aphid load in silver birch. New Phytologist 195:461-469.

Smillie, R. M. and S. E. Hetherington. 1999. Photoabatement by anthocyanin shields photosynthetic systems from light stress. Photosynthetica 36:451-463.

Smith, A. M. 1909. On the internal temperatures of leaves in tropical insolation, with special reference to the effect of their colour on temperature; also observations on the periodicity of the appearance of young coloured leaves of trees growing in Peradinaya Gardens. Annals of the Royal Botanical Garden. Peradinaya 5:229-297.

Smith, A. M. and M. Stitt. 2007. Coordination of carbon supply and plant growth. Plant, Cell \& Environment 30:1126-1149.

Smith, A. P. 1986. Ecology of a leaf color polymorphism in a tropical forest species habitat segregation and herbivory. Oecologia 69:283-287.

Soler, R., J. A. Harvey, A. F. D. Kamp, L. E. M. Vet, W. H. Van der Putten, N. M. Van Dam, J. F. Stuefer, R. Gols, C. A. Hordijk, and T. M. Bezemer. 2007. Root herbivores influence the behaviour of an aboveground parasitoid through changes in plant-volatile signals. Oikos 116:367-376.

Stamp, N. 2003. Out of the quagmire of plant defense hypotheses. The Quarterly Review of Biology 78:23-55.

Stapleton, A. E. and V. Walbot. 1994. Flavonoids can protect maize DNA from the induction of ultraviolet radiation damage. Plant Physiology 105:881-889.

Stephens, A. 2001. Pseudocoremia (Lepidoptera: Geometridae: Ennominae) systematics, biogeography and host plant associations. Victoria University of Wellington, Wellington, New Zealand. 
Steyn, W. J., S. J. E. Wand, D. M. Holcroft, and G. Jacobs. 2002. Anthocyanins in vegetative tissues: a proposed unified function in photoprotection. New Phytologist 155:349-361.

Steyn, W. J., S. J. E. Wand, G. Jacobs, R. C. Rosecrance, and S. C. Roberts. 2009. Evidence for a photoprotective function of low-temperature-induced anthocyanin accumulation in apple and pear peel. Physiologia Plantarum 136:461-472.

Stiles, E. W. 1982. Fruit flags - 2 hypotheses. American Naturalist 120:500-509.

Stone, B. C. 1979. Protective coloration of young leaves in certain Malaysian palms. Biotropica 11:126-126.

Stowe, K. A. and R. J. Marquis. 2011. Costs of defence: correlated responses to divergent selection for foliar glucosinolate content in Brassica rapa. Evolutionary Ecology 25:763-775.

Stowe, K. A., R. J. Marquis, C. G. Hochwender, and E. L. Simms. 2000. The evolutionary ecology of tolerance to consumer damage. Annual Review of Ecology and Systematics 31:565-595.

Strack, D. and V. Wray. 1989. Anthocyanins. Pages 325-356 in P. M. Dey and J. B. Harborne, editors. Methods in plant biochemistry. Academic Press, New York

Strauss, S. Y. and A. A. Agrawal. 1999. The ecology and evolution of plant tolerance to herbivory. Trends in Ecology \& Evolution 14:179-185.

Strauss, S. Y., R. E. Irwin, and V. M. Lambrix. 2004. Optimal defence theory and flower petal colour predict variation in the secondary chemistry of wild radish. Journal of Ecology 92:132-141.

Strauss, S. Y., J. A. Rudgers, L. J. A., and R. E. Irwin. 2002. Direct and ecological costs of resistance to herbivory. Trends in Ecology \& Evolution 17:278-285.

Suckling, D. M., G. Karg, A. R. Gibb, and S. J. Bradley. 1996. Electroantennogram and oviposition responses of Epiphyas postvittana (Lepidoptera: Tortricidae). New Zealand Journal of Crop and Horticultural Science 24:323-333.

Sun, J., J. N. Nishio, and T. C. Vogelmann. 1998. Green light drives CO2 fixation deep within leaves. Plant and Cell Physiology 39:1020-1026.

Sutherland, W. J., editor. 2006. Ecological census techniques: a handbook. Cambridge University Press, Cambridge.

Taulavuori, K., H. Pihlajaniemi, S. Huttunen, and E. Taulavuori. 2011. Reddish spring colouring of deciduous leaves: a sign of ecotype? Trees-Structure and Function 25:231-236.

Terashima, I., T. Fujita, T. TInoue, W. S. Chow, and R. Ogushi. 2009. Green light drives leaf photosynthesis more efficiently than red light in strong white light: revisiting the enigmatic question of why leaves are green. Plant and Cell Physiology 50:684-697.

Tholl, D., W. Boland, A. Hansel, F. Loreto, U. S. R. Röse, and J. P. Schnitzler. 2006. Practical approaches to plant volatile analysis. The Plant Journal 45:540-560.

Thompson, J. N. 2005. The geographic mosaic of coevolution. The University of Chicago Press, Chicago.

Tissue, D. T., K. L. Griffin, M. H. Turnbull, and D. Whitehead. 2005. Stomatal and non-stomatal limitations to photosynthesis in four tree species in a temperate rainforest dominated by Dacrydium cupressinum in New Zealand. Tree Physiology 25:447-456.

Townsend, J. A., B. Brown, I. A. N. Stringer, and M. A. Potter. 1997. Distribution, habitat and conservation status of Hemideina ricta and H. femorata on Banks Peninsula, New Zealand. New Zealand Journal of Ecology 21:43-49. 
Trewick, S. A. and M. Morgan-Richards. 1995. On the distribution of tree weta in the North Island, New Zealand. Journal of the Royal Society of New Zealand 25:485-493.

Turlings, T. C. J., U. B. Lengwiler, M. L. Bernasconi, and D. Wechsler. 1998. Timing of induced volatile emissions in maize sedlings. Planta 207:146-152.

Turlings, T. C. J., J. H. Tumlinson, and W. J. Lewis. 1990. Exploitation of herbivoreinduced plant odors by host-seeking parasitic wasps. Science 250:1251-1253.

Unsicker, S. B., G. Kunert, and J. Gershenzon. 2009. Protective perfumes: the role of vegetative volatiles in plant defense against herbivores. Current Opinion in Plant Biology 12:479-485.

Valero, E., M. J. Villaseñor, J. Sanz, and I. Martinez Castro. 2000. Comparison of two methods based on dynamic head-space for GC-MS analysis of volatile components of cheeses. Chromatographa 52:340-344.

van den Berg, A. K. and T. D. Perkins. 2007. Contribution of anthocyanins to the antioxidant capacity of juvenile and senescing sugar maple (Acer saccharum) leaves. Functional Plant Biology 34:714-719.

van den Berg, A. K., T. C. Vogelmann, and T. D. Perkins. 2009. Anthocyanin influence on light absorption within juvenile and senescing sugar maple leaves - do anthocyanins function as photoprotective visible light screens? Functional Plant Biology 36:793-800.

Vancanneyt, G., C. Sanz, T. Farmaki, M. Paneque, F. Ortego, P. Castañera, and J. J. Sánchez-Serrano. 2001. Hydroperoxide lyase depletion in transgenic potato plants leads to an increase in aphid performance. Proceedings of the National Academy of Sciences 98:8139-8144.

Vickers, C. E., J. Gershenzon, M. T. Lerdau, and F. Loreto. 2009. A unified mechanism of action for isoprenoids in plant abiotic stress. Nature Chemical Biology 5:283-291.

Wang, H., G. Cao, and R. L. Prior. 1997. Oxygen radical absorbing capacity of anthocyanins. Journal of Agricultural and Food Chemistry 45:304-309.

Wayman, K. A., P. J. de Lange, L. Larsen, C. E. Sansom, and N. B. Perry. 2010. Chemotaxonomy of Pseudowintera: Sesquiterpene dialdehyde variants are species markers. Phytochemistry 71:766-772.

Wearing, C. H. (editor) 1999. Brownheaded leafrollers. HortNET Bug Key internet database. AgResearch. http://www.hortnet.co.nz/key/keys/info/bhl-info.htm. (accessed Oct 2012)

Wei, J. and L. Kang. 2011. Roles of (Z)-3-hexenol in plant-insect interactions. Plant signaling \& behavior 6:369-371.

Wennersten, L. and A. Forsman. 2012. Population-level consequences of polymorphism, plasticity and randomized phenotype switching: a review of predictions. Biological Reviews 87:756-767.

Wheldale, M. 1916. The anthocyanin pigments of plants. Cambridge University Press, London.

White, J. D., C. M. Trotter, L. J. Brown, and N. Scott. 2000. Nitrogen concentration in New Zealand vegetation foliage derived from laboratory and field spectrometry. International Journal of Remote Sensing 21:2525-2531.

Whitman, D. W. and F. J. Eller. 1992. Orientation of Microplitis croceipes (Hymenoptera, Braconidae) to green leaf volatiles - dose-response curves. Journal of Chemical Ecology 18:1743-1753. 
Wilhelm, C. and D. Selmar. 2010. Energy dissipation is an essential mechanism to sustain the viability of plants: the physiological limits of improved photosynthesis. Journal of Plant Physiology 168:79-87.

Wilkinson, D. M., T. N. Sherratt, D. M. Phillip, S. D. Wratten, A. F. G. Dixon, and A. J. Young. 2002. The adaptive significance of autumn leaf colours. Oikos 99:402-407.

Willson, M. F. and W. G. Hoppes. 1986. Foliar 'flags' for avian frugivores: signal or serendipity? Tasks for vegetation science 15:55-69.

Winkel-Shirley, B. 2001. Flavonoid biosynthesis. A colorful model for genetics, biochemistry, cell biology, and biotechnology. Plant Physiology 126:485-493.

Wong, A. R. and D. S. Srivastava. 2011. Red abaxial coloring reduces herbivory in Columnea consanguinea. Ecotropica 16:93-99.

Woodall, G. S. and G. R. Stewart. 1998. Do anthocyanins play a role in UV protection of the red juvenile leaves of Syzygium? Journal of Experimental Botany 49:1447-1450.

Xu, Y.-X., X.-G. Sun, Z. He, X.-H. Liu, and F. Ge. 2006. Electroantennogram responses of Carcelia matsukarehae to the volatiles of Pinus massoniana damaged by Dendrolimus punctatus. Chinese Bulletin of Entomology 43:319322 .

Yamazaki, K. 2008a. Autumn leaf colouration: a new hypothesis involving plant-ant mutualism via aphids. Naturwissenschaften 95:671-676.

Yamazaki, K. 2008b. Colors of young and old spring leaves as a potential signal for ant-tended hemipterans. Plant signaling \& behavior 3:984-985.

Youard, L. 2012. The function of secondary metabolites in the leaves of Pseudowintera colorata. PhD. University of Otago, Dunedin.

Zaluki, M., A. R. Clarke, and S. B. Malcom. 2002. Ecology and behavior of first instar larval Lepidoptera. Annual Review of Entomology 47:361-393.

Zangerl, A. R., J. G. Hamilton, T. J. Miller, A. R. Crofts, K. Oxborough, M. R. Berenbaum, and E. H. de Lucia. 2002. Impact of folivory on photosynthesis is greater than the sum of its holes. Proceedings of the National Academy of Sciences 99:1088-1091.

Zeliou, K., Y. Manetas, and Y. Petropoulou. 2009. Transient winter leaf reddening in Cistus creticus characterizes weak (stress-sensitive) individuals, yet anthocyanins cannot alleviate the adverse effects on photosynthesis. Journal of Experimental Botany 60:3031-3042.

Zhang, K., X. Wang, J. Cui, J. Ogweno, K. Shi, Y. Zhou, and J. Yu. 2011. Characteristics of gas exchange and chlorophyll fluorescence in red and green leaves of Begonia semperflorens. Biologia Plantarum 55:361-364.

Zhang, Q., L.-J. Su, J.-W. Chen, X.-Q. Zeng, B.-Y. Sun, and C.-L. Peng. 2012. The antioxidative role of anthocyanins in Arabidopsis under high-irradiance. Biologia Plantarum 56:97-104. 